\begin{abstract}
Title of dissertation: Measurement of the Strange Quark Contribution to Nucleon Structure Through Parity-Violating Electron Scattering

Colleen Ellis, Doctor of Philosophy, 2010

Dissertation directed by: Professor Elizabeth J. Beise Department of Physics
\end{abstract}

The $G^{0}$ backward angle experiment, completed in Hall C of the Thomas Jefferson National Accelerator Facility (TJNAF), measured parity-violating asymmetries in elastic electron-proton and quasielastic electron-deuteron scattering at $Q^{2}=0.22$ and $0.63(\mathrm{GeV} / \mathrm{c})^{2}$. The asymmetries are sensitive to strange quark contributions to currents in the nucleon and the nucleon axial-vector current. The results indicate strange quark contributions of $\leq 10 \%$ of the charge and magnetic nucleon form factors at these four-momentum transfers. This was also the first measurement of the anapole moment effects in the axial-vector current at these four-momentum transfers. 


\title{
Measurement of the Strange Quark Contribution to Nucleon Structure Through Parity-Violating Electron Scattering
}

\author{
by \\ Colleen Ellis \\ Dissertation submitted to the Faculty of the Graduate School of the \\ University of Maryland, College Park in partial fulfillment \\ of the requirements for the degree of \\ Doctor of Philosophy \\ 2010
}

Advisory Committee:

Professor Elizabeth Beise, Chair/Advisor

Asst. Prof. Carter Hall

Dr. Herbert Breuer

Professor Thomas Cohen

Professor Michael Coplan 
(c) Copyright by Colleen Ellis 2010 


\section{Acknowledgments}

There are many people I wish to thank. My mother, Roberta Fay Ellis, who has been a source of boundless love, encouragement and inspiration. She herself earned an M.S. in Psychology Counseling, graduating with honors, at the age of 47 while working full time. Thank you for actively supporting me in my determination to pursue new challenges, now and always. To my brother Lantz Ellis, who besides being a good man and a fascinating person, has always had my back. To my father, Glenn Maurice Ellis, who I lost too soon, but who would have enjoyed my journey. To my dear friend and relative Rev. Robert B. Lantz who showered me with support and encouragement before he lost his brief but mighty battle with amyloidosis two years ago. To dear friend and relative Katherine Isenhour Lantz whose quiet wisdom and strength of character is a source of inspiration. I have two incredible sets of aunts, uncles, and cousins. All were an important part of my formative years, and all had a hand in who I've become-thank you for all of the love, support and wonderful memories: The Buckley's; Chris, Maurice, Tami and Rob and the Fowler's; Janice, Jack, Anita, Debbie and Laurie.

I have been blessed with incredible friends. They have all provided support and encouragement whenever needed and without them, my life would be far less rich and I would be far less interesting. Susan Kaye, Lisa and Andy Batchelor, Marcia Van Wye, John Egan, John and Kenne Lynch, Mary McKiel, Michael Kurtz, Cherie Loustaunau, Christine Villa, Charlie Moss, Kimberly Villa, Stephen Welby, Ann Kempski, Anne Patterson, Carol, Spryo, and Alexis Livanis, and Pamela "Kiki" 
Clifford.

Attending the University of Maryland at College Park has been an extraordinary experience. The caliber of the academic and research staff is eye-watering. A special thanks to Chris Lobb, who on my very first day of class at Maryland, while taking senior-level physics courses to fill a few holes in my background, asked the new students to stop by after class and introduce ourselves. When I did and explained my hope to attend grad school, he was encouraging, supportive, and full of practical advice. He played a key, pivotal role in my academic pursuit that I will always be grateful for.

A very special thanks to Phil Roos who taught me undergraduate quantum mechanics, and during one lecture, mentioned the $G^{0}$ experiment that was underway at Jefferson Lab. I made an appointment to see him to hear more about the opportunities in nuclear physics. He subsequently introduced me to Betsy Beise and to the $G^{0}$ collaboration.

My advisor, Betsy Beise, is a brilliant physicist, teacher, and mentor who is also a wonderful, and interesting person. She has taught me so much, and not just physics. I can't thank her enough for the education and opportunities that she has provided to me, and also for her patience.

My time at Maryland has been a rewarding experience, and this is due in no small part to the other students in the experimental nuclear physics group. Thanks to Tanya Horn and Jianglai Liu who were Betsy's students when I arrived and who both provided me with help and support well after they graduated. Thanks too to Simon Slutsky, Tom Langford, Attila Dobi, Yung Ruey Yen, Prabin Adhikari, 
Pat Harding, and Jon Miller as well as undergraduates Norvik Voskanian, Clint Richardson, Jon Hood, Dylan Erwin, Wes Szamotula, and Seth Toplosky.

Also a part of the experimental physics group at Maryland was a very talented and helpful group of postdocs; Doug Leonard, Lisa Kaufman and Kaz Nakahara. Special recognition and thanks to Fatiha Benmokhtar who was not only a Maryland postdoc, but the analysis coordinator for the $G^{0}$ backward angle measurement.

A special thanks to Herbert Breuer, who helped me with me the luminosity detectors and with the neutron tests at NIST. I learned an incredible amount of practical, experimental techniques from Herbert, and had a great deal of fun while doing so.

Thank you to all of my committee members, Betsy, Herbert, Carter Hall, Michael Coplan, and Tom Cohen for graciously agreeing to be on my committee and review this work.

The $G^{0}$ collaboration was full of talented physicists led by spokesman Doug Beck. He provided us with vision, leadership, and support in all of our endeavors. A special thanks to Paul King for all of his help and patience on so many endeavors. Thanks to everyone from Hall C at Jefferson Lab, and in particular Dave Gaskell and Greg Smith.

Part of what made my thesis experience so positive were the other students I worked with. I enjoyed seeing everyone's home institution during our quarterly analysis group meetings when I had an opportunity to get to know everyone a little bit better. They are a fun, helpful, talented, hardworking, and fascinating group of people. Very special thanks to Mat Muether, Maud Versteegen, Carissa Capuano, 
John Schaub, Juliette Mammei, Alex Coppens, and Stephanie Bailey.

And to Chris O'Connor, for his support and encouragement and for showing me that the whole, can indeed, be far greater than the sum of the parts. 


\section{Table of Contents}

List of Tables $\quad$ ix

List of Figures $\quad$ xiii

1 Physics Motivation 1

1.1 Introduction . . . . . . . . . . . . . . . . . . . . 1

1.2 Strangeness in the Nucleon . . . . . . . . . . . . . . . . . . . 3

1.3 Strangeness Contribution to the Nucleon's Momentum . . . . . . . . 4

1.3.1 Strangeness Contribution to the Nucleon Mass . . . . . . . . . 4

1.3.2 Strangeness and Nucleon Spin . . . . . . . . . . . . . 6

1.3.3 Vector Strange Quark Matrix Elements . . . . . . . . . . 8

2 Theory and Formalism 10

2.1 Nucleon Form Factors . . . . . . . . . . . . . . . . . . . . . 11

2.1.1 Flavor Decomposition . . . . . . . . . . . . . 14

2.1.1.1 Flavor Vector Form Factors . . . . . . . . . . . . . . 15

2.1.1.2 Flavor Axial Form Factors . . . . . . . . . . . . . 17

2.1.1.3 Flavor Singlet Form Factors . . . . . . . . . . . . . 18

2.2 Parity Violation in Electron Scattering . . . . . . . . . . . . . . . 19

2.2.1 Experimental Observables: Neutral Weak Vector Form Factors 19

2.2.2 Electroweak Radiative Corrections to the Neutral Current . . 22

2.3 Axial Form Factor and the Anapole Moment . . . . . . . . . . . . . . 25

2.3.0.1 The Anapole Contribution . . . . . . . . . . . . 27

2.4 The Deuteron . . . . . . . . . . . . . . . . . . . 28

2.4.0.2 Two Boson Exchange Correction . . . . . . . . . . 29

2.5 Electroweak Parameters . . . . . . . . . . . . . . . . 31

2.6 Theoretical Predictions of $G_{E}^{S}$ and $G_{M}^{S} \ldots \ldots \ldots \ldots$

2.6.1 Chiral Perturbation Theory . . . . . . . . . . . . . 34

2.6.1.1 Loop Models . . . . . . . . . . . . . . . . 35

2.6.1.2 Pole Models . . . . . . . . . . . . . . . . . . . . 37

2.6.1.3 Loops and Poles . . . . . . . . . . . . . . 39

2.6 .2 Lattice QCD . . . . . . . . . . . . . . . . 40

3 Summary of Existing Measurements 42

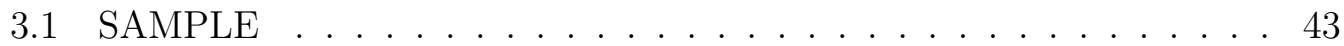

3.2 HAPPEx . . . . . . . . . . . . . . . . . . . 47

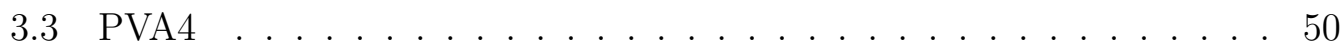

$3.4 G^{0}$ Forward Angle . . . . . . . . . . . . . . . . . . . . 55

3.5 Summary of Parity-Violating Measurements Prior to $G^{0}$ Backward

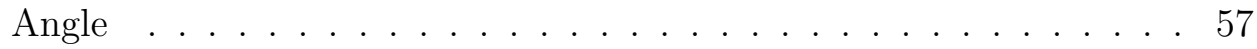


4 The $G^{0}$ Backward Angle Experimental Apparatus $\quad 60$

4.1 Overview . . . . . . . . . . . . . . . . . . . 60

4.2 Forward Angle Apparatus . . . . . . . . . . . . . . . . . . . . 63

4.3 Superconducting Magnet System . . . . . . . . . . . . . . . . . . . . 64

4.4 Focal Plane Detectors . . . . . . . . . . . . . . . . . . . . . 67

4.5 Cryostat Exit Detectors . . . . . . . . . . . . . . . 69

4.6 Čerenkov Detectors . . . . . . . . . . . . . . . . . . 70

4.7 Electronics . . . . . . . . . . . . . . . . . . . . . . . 73

4.8 Data Acquisition . . . . . . . . . . . . . . . . . . . . 77

4.9 The $G^{0}$ Polarized Electron Beam . . . . . . . . . . . . . . . . . 78

4.9 .1 CEBAF Accelerator . . . . . . . . . . . . . 80

4.9 .2 Polarized Source . . . . . . . . . . . . . . . . . . . 82

4.9.3 Beam Monitors . . . . . . . . . . . . . . . 85

4.9 .4 Polarimetry . . . . . . . . . . . . . . . . . 88

4.10 Target . . . . . . . . . . . . . . . . . . . . 92

5 Data Analysis $\quad 98$

5.1 Data Reduction Procedure . . . . . . . . . . . . . . . . . . . . . 98

5.1.1 $G^{0}$ Replay Engine . . . . . . . . . . . . . . . . . . 100

5.1.2 $G^{0}$ Backward Angle Data . . . . . . . . . . . . . . . 103

5.2 Corrections to the Raw Asymmetries . . . . . . . . . . . . . . . 110

5.2.1 Scaler Counting Rate Error Correction . . . . . . . . . . . . . 112

5.2 .2 Electronics Rate Correction . . . . . . . . . . . . . . . . . . 114

5.2 .3 Linear Regression . . . . . . . . . . . . . . . . . . . . . . . 124

5.2 .4 Dilution Correction . . . . . . . . . . . . . . . . . 129

5.2 .5 Polarization Correction . . . . . . . . . . . . . . . 132

5.2.6 Transverse Polarization . . . . . . . . . . . . . . . . . . . 136

5.2.7 Electromagnetic Radiative Corrections . . . . . . . . . . . . . 138

5.2.8 $Q^{2}$ Determination . . . . . . . . . . . . . . . . 141

5.3 Final Measured and Corrected Asymmetries . . . . . . . . . . . . . . 142

6 Results and Conclusions 145

6.1 Combining $G^{0}$ Forward and $G^{0}$ Backward Angle Measurements . . . . 145

6.2 Electromagnetic Form Factor Parameterization . . . . . . . . . . . . . 148

6.3 Asymmetry Coefficients . . . . . . . . . . . . . . . . 151

6.4 Results . . . . . . . . . . . . . . . . . . . . . . 157

6.4 .1 Conclusions . . . . . . . . . . . . . . . . . 159

6.5 Future Outlook . . . . . . . . . . . . . . . . . 160

A Electromagnetic Radiative Corrections 163

A.1 Introduction . . . . . . . . . . . . . . . . . . . . 163

A.2 GEANT Monte Carlo Simulation . . . . . . . . . . . . . . . 165

A.3 Details of the Calculation for Hydrogen . . . . . . . . . . . . . . 168

A.4 Details of the Calculation for Deuterium . . . . . . . . . . . . . 174

A.5 Results . . . . . . . . . . . . . . . . . . . . 178 
B Luminosity Monitors $\quad 184$

B.1 Detectors . . . . . . . . . . . . . . . . . . . . . 184

B.2 Geometry and Kinematics . . . . . . . . . . . . . . . 187

B.3 Detector Linearity . . . . . . . . . . . . . . . . . . . . . . . . . 196

B.4 Asymmetry Widths and Target Density Fluctuations . . . . . . . . . 197

B.5 Spin Calculation . . . . . . . . . . . . . . . . . . 204

C The $G^{0}$ Database 208

C.1 Introduction . . . . . . . . . . . . . . . . . . . . 208

C.2 Database Design and Structure . . . . . . . . . . . . . 208

$\begin{array}{ll}\text { Bibliography } & 217\end{array}$ 


\section{List of Tables}

1.1 Comparison of Sum Rule predictions including higher order corrections [LV91] with a global analysis of E155 results $\left[A^{+} 06 d\right]$ for $Q^{2}=5$ $(G e V / c)^{2}[F J 01] \ldots \ldots \ldots \ldots$

2.1 Electroweak charges of elementary fermions $\left[\mathrm{M}^{+} 94\right] \ldots \ldots$

2.2 The "one quark", "many quark", and total corrections to the axial charges in the $\overline{M S}$ scheme. . . . . . . . . . . . . . . . . 28

2.3 Parameters used to calculate the electroweak radiative corrections. . . 32

2.4 Radiative correction factors ("one quark"). . . . . . . . . . . . . . 33

2.5 Summary of theoretical predictions for $\mu_{s} s \ldots \ldots$. . . . . . . . . . . 41

3.1 A summary of world data prior to the $G^{0}$ backard angle measurement for a linear combination of strange vector form factors, $G_{E}^{s}+\eta G_{M}^{s}$, and the associated kinematics. The first and second asymmetry uncertainties are statistical and systematic, respectively. The targets are hydrogen or helium $(\eta=0) . \ldots \ldots$. . . . . . . . . 57

5.1 Blinding factors applied to the raw asymmetries. . . . . . . . . . . 100

5.2 The run periods showing target type, beam energy, and beam current. 110

5.3 Correction applied to the normalized measured rate of electrons in the elastic or quasi-elastic region as a function of octant [Ver09]. . . . . 120

5.4 Final false asymmetry and systematic error assigned to the electronics rate correction for elastic (quasi-elastic) electrons shown for each data set [Ver09]. . . . . . . . . . . . . . . . . . . . . . . . 123

5.5 Corrections to the raw measured asymmetry due to corrections to the yield to account for electronics effects for each target and beam energy along with the associated uncertainty $\left[\mathrm{Aac}^{+}\right.$10]. . . . . . . . . . . . . 124

5.6 The measured and maximum allowed charge asymmetry or beam parameter difference for the $G^{0}$ backward angle measurement. . . . . . . 125 
5.7 Calculated false asymmetries due to helicity-correlated beam parameters. The run period includes the target, beam energy, and if it does not include the entire time period, the timeframe of the data collection is also included. [Sch08] . . . . . . . . . . . . . . . . . . . 129

5.8 The primary contributions to the elastic (quasi-elastic) electron background where $f_{\text {other }}$ represents contributions from processes not otherwise listed and consists primarily of inelastic electrons and $\pi^{\circ}$ decay [Mue09]. . . . . . . . . . . . . . . . . . . . . 132

5.9 Correction to the measured asymmetry to neutralize the contribution of the background on the physics asymmetry. The uncertainties include the associated overall-all point-to-point and global systematic uncertainties.[Mue09] . . . . . . . . . . . . . . . . . 133

5.10 Magnitudes of the measured electron asymmetry during transverse beam polarization, luminosity monitor asymmetries during both longitudinal and transverse polarization, and the magnitude of the false asymmetry, $K_{T}$, due to a residual transverse component of the beam polarization for each of the data sets [Mam10]. . . . . . . . . . . . 138

5.11 Electromagnetic radiative corrections for each $G^{0}$ backward angle measurement target and energy combination. . . . . . . . . . . . . . . . 140

$5.12 Q^{2}$ for each data set during the backward angle measurement [Mam09a]. 142

5.13 The raw, unblinded, measured asymmetry by target, beam energy, and $Q^{2}$. The uncertainty is purely statistical. The data have received the cut on events that are $5 \sigma$ or more away from the mean yield value (pass 2 of the replay engine), but no other corrections. . . . . . . . . 142

5.14 Corrections to the raw asymmetries (Table 5.13). These corrections are additive. "Other" includes corrections for helicity-correlated beam parameters, the small transverse component of beam polarization, and two-boson exchange. The uncertainties are point-to-point and global systematic $\left[\mathrm{Aac}^{+}\right.$10]. . . . . . . . . . . . . . . . . . . . 143

5.15 Corrections to the raw asymmetries (Table 5.13). These corrections are multiplicative. They include the beam polarization $(1 / P)$ and the correction for electromagnetic (EM) radiative effects. The uncertainties are point-to-point and global systematic [Aac ${ }^{+}$10] . . . . . . . 143

5.16 The final physics asymmetries from the $G^{0}$ backward angle measurement. The uncertainties are statistical, point-to-point, and global systematic $\left[\right.$ Aac $^{+}$10] . . . . . . . . . . . . . . . . 144 
6.1 The final physics asymmetries from the $G^{0}$ backward angle measurement. The uncertainties are statistical, point-to-point, and global systematic $\left[\mathrm{Aac}^{+}\right.$10]. . . . . . . . . . . . . . . . . 146

6.2 The final physics asymmetries from the $G^{0}$ backward angle measurement adjusted to the common $Q^{2}$ values. The uncertainties are statistical, point-to-point, and global systematic [Aac ${ }^{+}$10]. . . . . . . 146

6.3 The $G^{0}$ forward angle physics asymmetries interpolated to $Q^{2}$ values of the backward angle measurement. . . . . . . . . . . . . . . 148

6.4 Radiative correction factors, including the baryon beta decay param-

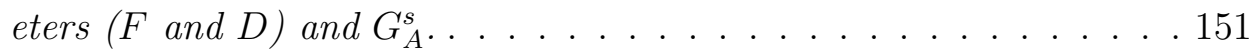

6.5 The standard parameters used in the form factor extraction calculation. The uncertainties are either negligible or as in the case of $\Lambda$, included in the overall form factor uncertainties. . . . . . . . . . . . 153

6.6 Values of the parameters used in the calculation of the deuterium asymmetry coefficients [Bei08]. . . . . . . . . . . . . 156

6.7 The measured asymmetries, adjusted to the common $Q^{2}$ values, and the corresponding calculated asymmetry coefficients, for both the forward angle (hydrogen target) and the backward angle measurements (hydrogen and deuterium targets). The uncertainties are statistical, point-to-point systematic, and global systematic uncertainties, respectively. . . . . . . . . . . . . . . . . . 157

6.8 Extracted vector strange and axial form factors obtained from the measurements taken during the $G^{0}$ forward angle and backward angle measurements. The first set of uncertainties are associated with the asymmetry measurement. The second set of uncertainties are related to the parameters used to extract the form factors from the measurement. . . . . . . . . . . . . . . . . . 159

A.1 Deuterium Electromagnetic Radiative Corrections. Multiplicity $=2$ unless otherwise noted. . . . . . . . . . . . . . . . . . . . . . 182

A.2 Hydrogen Electromagnetic Radiative Corrections. Multiplicity = 2. . . 183

B.1 Luminosity values for all of the target and beam current combinations used during the $G^{0}$ backward angle measurement. . . . . . . . . . . . . 188 
B.2 Predicted luminosity monitor yields and asymmetries by reaction channel and luminosity monitor for a hydrogen target. Beam energy is 360 MeV and beam current is $60 \mu \mathrm{A}$. . . . . . . . . . . . . . . . . . . 189

B.3 Predicted luminosity monitor yields and asymmetries by reaction channel and luminosity monitor for a hydrogen target. Beam energy is 685 MeV and beam current is $60 \mu \mathrm{A}$. . . . . . . . . . . . . . . . 189

B.4 Predicted luminosity monitor yields and asymmetries by reaction channel and luminosity monitor for a deuterium target. Beam energy is $360 \mathrm{MeV}$ and beam current is $35 \mu \mathrm{A}$. . . . . . . . . . . . . . . 192

B.5 Predicted luminosity monitor yields and asymmetries by reaction channel and luminosity monitor for a deuterium target. Beam energy is $685 \mathrm{MeV}$ and beam current is $20 \mu \mathrm{A}$. . . . . . . . . . . . . . 192

B.6 Spin angle for nominal longitudinally polarized electron beam calculated using Lumi asymmetries. . . . . . . . . . . . . . . . . . . . . 206 


\section{List of Figures}

2.1 Total leading order amplitude for electron scattering from a nucleon is the sum of the leading order electromagnetic and neutral current amplitudes. . . . . . . . . . . . . . . . . 10

2.2 Representative one-quark Feynman diagrams contributing to electroweak radiative corrections. . . . . . . . . . . . . . . . 23

2.3 The primary Feynman diagram for a loop-model calculaton. A nu-

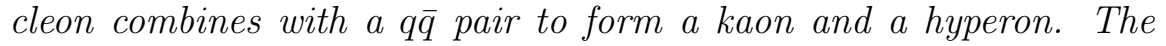
$q \bar{q}$ pair annihilates when the kaon and hyperon recombine leaving the original nucleon. . . . . . . . . . . . . . . . . . . 35

2.4 The primary Feynman diagram for a pole-model calculaton. . . . . . . 38

2.5 Three point function representations. The connected insertion (left) compared to the disconnected insertion (right) $\left[D^{+} 09 a\right] . . . . . . .440$

3.1 Depiction of the SAMPLE experimental apparatus. The Čerenkov photons from backward-scattered electrons were reflected and focused by the mirrors onto the photomultiplier tubes. . . . . . . . . . . . . . 44

3.2 Uncertainty bands of $G_{M}^{s}$ and $G_{A}^{e(T=1)}$ at $Q^{2}=0.1(G e V / c)^{2}$ resulting from the two $200 \mathrm{MeV} S A M P L E$ data sets $\left[S^{+} 04\right.$, BPS05] labelled " $\mathrm{H}_{2}$ and $\mathrm{D}_{2}$ ". Also shown is the uncertainty band of the theoretical expectation of $G_{A}^{e(T=1)}$ as computed by [ZPHRM00], labelled as "Zhu, et al.", extrapolated to the same momentum transfer. The smaller ellipse corresponds to the $1 \sigma$ overlap of the hydrogen data and the theoretical prediction; the larger one the $1 \sigma$ overlap of the two data sets [S+04]. The inner dashed line $\left(H_{2}\right)$, and inner straight lines $\left(D_{2}\right.$ and $Z$ hu, et al) represent the $2 \sigma$ confidence bands. . . . . . . . . . . 45

3.3 The physics asymmetries measured in SAMPLE II and SAMPLE III are plotted as a function of $Q^{2}$ (solid circles). Also plotted (with offset $Q^{2}$ for visibility) is the theoretical prediction using $G_{A}^{e}$ from [ZPHRM00] and $G_{M}^{s}=0.15$ (open circles). The height of the gray rectangles represents the change in the physics asymmetry corresponding to a 0.6 change in $G_{M}^{s}\left[I^{+} 04\right]$. . . . . . . . . . . . . . 46

3.4 A depiction of the HAPPEx apparatus, used for all of the measurements from $\left[A^{+} 04\right]$. Forward scattered electrons are detected by the high-resolution spectrometers. . . . . . . . . . . . . . 48

3.5 PVA4 target, calorimeter, and luminosity monitors [Rio09]. . . . . . 51 
3.6 Data from PVA4. Forward angle data on the left (a) shows the elastic peak clearly and a distinct background dominated by $\pi^{\circ}$ and gammas. The data on the right from the backward angle running (b) shows the elastic electron peak at the same energy as $\pi^{\circ}$ and gamma background. [Rio09]. . . . . . . . . . . . . . . . . 52

3.7 Drawing of the PVA4 $\mathrm{PbF}_{2}$ calorimeter in the backward angle configuration. The scintillators are placed between the scattering chamber and the lead fluoride crystals. $\left[B^{+} 09 a\right] . \ldots \ldots . . \ldots . . . . .53$

3.8 Separation of the elastic peak from the neutral particles through the use of scintillators that detect charged particles for the PVA4 experiment [Rio09]. . . . . . . . . . . . . . . . . . . . 53

3.9 The results of the $G^{0}$ forward angle measurement as a function of four-momentum transfer $\left[A^{+} 05\right]$. The error bars include the statistical uncertainty (inner) and statistical plus point-to-point uncertainties (outer). The grey error bands represent the global systematic uncertainties $\left(G^{0}\right.$ only). The upper band shows the global uncertainties from the measurement and the lower band incorporates the theoretical uncertainties used to calculate $A_{N V S}$. HAPPEx results $\left[A^{+} 06 c, A^{+} 06 b, A^{+} 07\right]$ are shown as diamonds for comparison. . . . 56

3.10 World data constraints on $\left(G_{E}^{s}, G_{M}^{s}\right)$ at $Q^{2}=0.1(G e V / c)^{2}$. The Kelly form factors [Kel04] are used. Different bands in the plot represent: SAMPLE-H [S+00] (solid red), SAMPLE-D [I+ $\mathrm{I}^{+}$] (dashed red), HAPPEx-H-a [A+99b] (dashed blue), Happex-H-b [A+01] (solid blue), Happex-he-a [A+06b] (dashed pink), Happex-he-b [A+07] (solid pink), $\mathrm{PVA} 4-\mathrm{H}\left[\mathrm{M}^{+} 05\right]$ (solid green), and $\mathrm{G}^{0}$ forward angle $\left[\mathrm{A}^{+} 05\right]$ (solid brown). The yellow and grey ellipses represents $68.27 \%\left(\Delta \chi^{2}=\right.$ 2.3 ) and $95 \%\left(\Delta \chi^{2}=5.99\right)$ confidence contours around the point of maximum likelihood at $G_{E}^{s}=-0.004, G_{M}^{s}=0.30$. The black cross represents $G_{E}^{s}=G_{M}^{s}=0$. [LMRM07]. . . . . . . . . . . . . 59

4.1 A schematic drawing of the $G^{0}$ spectrometer including the SMS and detectors mounted on the ferris wheel. . . . . . . . . . . . . . . . . 61

4.2 A schematic drawing of the particle trajectory from the target to the detectors in the $G^{0}$ spectrometer for the backward angle measurement. Only one of the eight detector sets is depicted. . . . . . . . . . . . . . 62

4.3 A schematic of the particle trajectory from the target to the detectors in the $G^{0}$ spectrometer for the forward angle measurement. Only one of the eight detector sets is depicted. . . . . . . . . . . . . . 63 
4.4 A schematic drawing of the eight superconducting coils viewed from the target and looking upstream (backward angle mode). The magnetic field direction is shown by the green arrows. The layout of the coils, (A-H) and the detector octants (1-8) is shown. The beam is located at the cross in the center. . . . . . . . . . . . . . . . . . 65

4.5 SMS collimators shown for one octant [Bat04] . . . . . . . 66

4.6 Focal Plane Detectors (FPDs) for one octant. . . . . . . . . . . . 67

4.7 Cryotstat Exit Detector (CED) and support structure for one octant. 70

4.8 Čerenkov design including magnetic shielding [Gui05] . . . . . . . . 71

4.9 Schematic drawing of the NA electronics. . . . . . . . . . . . . 75

4.10 Schematic drawing of the French electronics. . . . . . . . . . . . . 76

4.11 A block diagram of the $G^{0} D A Q$ system. ROCO contained the trigger supervisor (TSO), and the beam monitor scalers. ROC1 contained NA coincidence scalers. ROC 2 contained 10 NA coincidence scalers, NA ARS and Čerenkov scalers. ROC3 contained eight French DMCH boards. ROC4 held $19 \mathrm{NA}$ coincidence scalers modules. ROC5 contained the Fastbus TDC and ADC modules. ROC7 contained the French CED-FPD coincidence boards, ROC8 contained the French Cerenkov modules and the French ARS. ROC9 contained the NA singles scalers. ROC31 contained the beam injector data. Several modules not identified here are described in [Ben06]. . . . . . . . . . . . 79

4.12 A schematic drawing of the JLab CEBAF accelerator [Gra06]. . . . . 80

4.13 A schematic drawing of the JLab CEBAF injector [Gra06] . . . . . . 81

4.14 A simplified schematic drawing of the $G^{0}$ laser table. . . . . . . . . . 84

4.15 A diagram of a BPM, showing the four strip lines running along the inside of the cylinder [BaiO7]. . . . . . . . . . . . . . . 87

4.16 The layout of the Hall C Møller polarimeter . . . . . . . . . . . . . . 89

4.17 The layout of the Hall C Mott polarimeter. The beam direction is from left to right. . . . . . . . . . . . . . . . . . . 91

4.18 A schematic drawing of the $G^{0}$ target loop. . . . . . . . . . . . . . . 92

4.19 The target service module with the target in the "in beam" position. $[\mathrm{Co} 05] \ldots \ldots \ldots . \ldots \ldots . \ldots \ldots$ 
4.20 A schematic drawing [Co05] and photograph of the $G^{0}$ target cell and manifold. . . . . . . . . . . . . . . . . 95

5.1 A diagram of the data flow for the $G^{0}$ replay engine. . . . . . . . . . 102

5.2 Normalized yield data in $H z / \mu A$ for each of the $G^{0}$ backward angle target and energy combinations. Data for only octant is shown. The vertical axis are the CEDs and the horizontal axis the FPDs. The detectors with the smallest number are located closest to the target. The cells outlined in black represents the elastic (quasi-elastic) locus. 104

5.3 Cell by cell fits using a Gaussian (blue) for low momentum background and 2 Gaussians (with shared width) (red) for the elastic peak. A constant (lt. green) is also added to the fit to remove any field independent rate. The vertical black line is the nominal SMS setting. . . . 106

5.4 The various kinematic regions for the two beam energies as represented by CED - FPD detector pairs. CED number is on the vertical axis, FPD number on the horizontal axis. Red represents the cells dominated by elastic electrons, while the blue region is mostly inelastic electrons. Super-elastic is a region that should be inaccessible to electron scattering from the target, while the background region (green) is where various processes contribute to the rate, including those scattered from target walls. The black cells have a combination of particles from adjacent regions. . . . . . . . . . . . . . . 107

5.5 Typical asymmetry plots as a function of octant for electrons scattered elastically from a hydrogen target. No corrections have been made to the data, and the asymmetry values have the blinding factor applied.[Ver09] . . . . . . . . . . . . . . . . . . . . . 109

5.6 Typical asymmetry plots as a function of octant for electrons scattered quasi-elastically from a deuterium target. No corrections have been made to the data, and the asymmetry values have the blinding factor applied.[Ver09] . . . . . . . . . . . . . . . . . 109 
5.7 A diagram of the analysis process. A blinding factor is applied to the raw, uncorrected asymmetries. The raw yields, from which the raw asymmetries are formed, receives specific corrections during each of the 4 analysis "passes". After the 4 th pass, the corrected yields generate the "measured" asymmetries, which are still blinded. The measured asymmetry is corrected for background events and dilutions before the blinding factor is removed. The electromagnetic radiative corrections and the correction for the beam polarization is applied to $A_{\text {meas }}$, generating $A_{\text {phy }}$, which is used to extract the vector strange and axial form factors. . . . . . . . . . . . . . . . . . . . 111

5.8 The cause of the bit error in the scaler that recorded a CED-FPD coincidence. If two mean timer FPD signals separated by 2 ns were within the trigger window of a CED mean timer signal, two 7 ns pulses would be sent to the scaler, instead of one 10 ns signal resulting in a complicated pattern of bit inversion errors [Rea08]. . . . . . . . . . . 113

5.9 The plot shows the yield in $k H z / \mu A$ for one CED-FPD detector pair during the low energy running with a deuterium target. The nonGaussian distribution shown in black and blue around the mean yield, indicates a problem with the electronics. The result of cutting all events $5 \sigma$ or more away from the mean distribution is shown in red. The cut is on the yield and no cuts were made to the asymmetries. . 114

5.10 The measured and corrected rate for $e+\pi$ (all CED $\times$ FPD coincidences with or without a Čerenkov hit) versus beam current. The corrections account for electronics dead time and random events. The data were from a hydrogen target at $687 \mathrm{MeV}$ for two cells affected primarily by one correction or the other. The cell on the left is in the background region. The cell on the right is in the elastic region. [Ver09]118

5.11 Correlation slopes, $\partial Y / \partial x_{i}$, plotted as a function of run number, where $x_{i}$ is a beam parameter for the running on a deuterium target at $362 \mathrm{MeV} \ldots \ldots$. . . . . . . . . . . . . . . . . . . . . . 128

5.12 Magnet current scan for a single elastic cell on the inelastic edge of the elastic locus [Mue09]. The dotted vertical line is the nominal SMS current setting. All data points, except those labelled "measured", are from scaled Monte Carlo simulations. . . . . . . . . . . . . . . . . . 130

5.13 Møller polarization measurements taken during the 687 MeV running. Inner error bars denote statistical errors only, while the outer error bars denote statistical and point-to-point added in quadrature. The solid line is a constant fit using the outer error bars, and the dashed lines are the uncertainty on the fit [GH08]. . . . . . . . . . . . . . 134 
5.14 Mott measurements during the $G^{0}$ backward angle measurement. Only statistical errors are shown.[GH08]. . . . . . . . . . . . . . . . 136

5.15 Simulated photon background assuming the beam dump was a $21 \mathrm{~mm}$ thick aluminum plate[GH08]. . . . . . . . . . . . . . . . . 137

5.16 Luminosity Monitor asymmetries versus octant for each target/energy combination. Octant 1 was located at the 12 o'clock position when looking down the beam line, with the other octants placed in a clockwise manner with increasing number. The sine fits show the luminosity monitor sensitivity to any transverse component of the beam polarization. The fit parameters are p0 (amplitude), p1 (phase shift) and p2 (constant offset). . . . . . . . . . . . . . . . 139

6.1 The physics asymmetries measured during the forward angle portion of the $G^{0}$ experiment which used a hydrogen target. The inner error bars are the statistical uncertainty, and the outer error bars are the statistical and point-to-point systematic uncertainties combined in quadrature. The global uncertainty, $\Delta A_{\text {glob }}$, is shown at the top of the graph in gray. $A_{N V S}$, is shown as the solid gray line. . . . . . . . . . 147

6.2 Fits for the electromagnetic form factors of the neutron and proton with a range of $Q^{2}$ from 0.1 to $1.1(\mathrm{Gev} / \mathrm{c})^{2}$. $G_{E}^{p}, G_{M}^{p}$, and $G_{E}^{n}$ were normalized using their corresponding dipole fits. The data and the fit for Kelly (solid blue) are from [Kel04]. The other curves in the figures represent different form factor parameterizations: (pink dot-dashed $=$ [Arr04], black dashed = Friedrich-Walcher [FW03].) The Arrington parameterization is for the proton only. This figure is taken from [Liu06]. . . . . . . . . . . . . . . . . . . . . 149

6.3 Results of $\mu G_{E}^{p} / G_{M}^{p}$ shown with the world polarized data and several models, including the Kelly parameterization $\left[C^{+} 0^{r} 7\right] . \ldots 150$

6.4 The form factors determined by the $G^{0}$ forward and backward angle measurements. Error bars show statistical and statistical plus point-to-point systematic uncertainties; shaded bars below corresponding points show global systematic uncertainties $\left(G^{0}\right)$. For $G_{E}^{s}$ and $G_{M}^{s}$, the extraction from [LMRMO7] (Low $Q^{2}$ fit) and the PVA4 experiment $\left[B^{+} 09 b\right]$ are shown. Recent calculations from Adelaide $\left[L B C^{+} 05, L B T^{+} 06\right]$ and Kentucky $\left[D^{+} 096\right]$ are also shown. The Adelaide uncertainties are smaller than the symbols. For $G_{A}^{e, T=1}$, results from the SAMPLE experiment [BPS05] are shown with the calculation of Zhu, et al. [ZPHRMO0] . . . . . . . . . . . . . 158 
A.1 First order diagrams for the elastic scattering of an electron from a nucleon. . . . . . . . . . . . . . . . . . 164

A.2 Next to leading order Feynman diagrams necessary to calculate electromagnetic radiative effects . . . . . . . . . . . . . . . . . 165

A.3 $Q^{2}$ vs normalized event rates for incident beam energy $685 \mathrm{MeV}$ and a hydrogen target. The plot on the left is with no radiative effects, the plot on the right includes radiative corrections. . . . . . . . . . . . . . 180

A.4 $a_{0}$ vs normalized event rates for incident beam energy $685 \mathrm{MeV}$ and a hydrogen target. The plot on the left is with no radiative effects, the plot on the right includes radiative corrections. . . . . . . . . . . . . . 180

B.1 An illustration of the placement of the Lumis in relationship to one another and the beam line. . . . . . . . . . . . . . . . . . . . 184

B.2 An illustration of the configuration of one Lumi detector, not drawn to scale. The quartz scintillator, copper mesh, and PMT, for each detector were located in the experimental hall. The electronics, which included the I-V preamp, V-F convertors, and the scalers were located in the $G^{0}$ electronics cage. . . . . . . . . . . . . . . . . . . . . . . 185

B.3 An illustration of the placement of one set of four Lumis around the beam pipe viewed from the target. A cross section of the downstream beam pipe, the detectors and the aluminum cups that hold the detectors are shown. The beam was centered on the axis of the beam pipe, and $19.8 \mathrm{~cm}$ from the bottom of each aluminium cup. . . . . . . . . . . . 187

B.4 Electron yield versus laboratory scattering angle for the Lumi solid angle for a hydrogen target with aluminum target windows, shown by reaction channel. Processes include Møller, e-p, and elastic e-Al scattering. . . . . . . . . . . . . . . . . . 190

B.5 Electron yield versus laboratory scattering angle for the Lumi solid angle for a deuterium target with aluminum target windows, shown by reaction channel. Processes include Møller, e-n, and elastic e-Al scattering. . . . . . . . . . . . . . . . . . . . . 191

B.6 Customized base designed for the Hamamatsu R375 photomultiplier tube. Only the first four stages are active. The signal is extracted at the 5th dynode stage. The resistors are in ohms and kilohms (k). . . 195

B.7 The estimated gain versus high voltage for the Hamamatsu R375 photomultiplier tube with a customized low gain base. . . . . . . . . . . . 195 
B.8 Beam current normalized yields versus beam current for a hydrogen

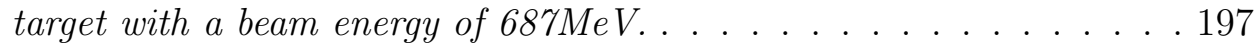

B.9 Measured asymmetry widths for a carbon target at 687MeV. A counting statistics fit for Lumi3 is shown. . . . . . . . . . . . . . . . . . . 199

B.10 Measured asymmetry widths for a liquid deuterium target at $687 \mathrm{MeV}$. A counting statistics fit for Lumi3 is shown. The difference between the fit and the data can be attributed to target density fluctuations. . . 201

B.11 Measured asymmetry widths for a liquid hydrogen target at $687 \mathrm{MeV}$. A counting statistics fit for Lumi3 is shown. The difference between the fit and the data can be attributed to target density fluctuations. . . 202

B.12 Illustration of the numbering of the octants (numbers inside the circle) and the corresponding Lumi monitor. The beam is going into the page.204

B.13 Lumi asymmetries (blinded) versus octant for longitudinally polarized electrons scattered at $687 \mathrm{MeV}$ from an unpolarized hydrogen target. 205

B.14 Lumi asymmetries (blinded) versus octant for transversely polarized electrons scattered at $68^{7} \mathrm{MeV}$ from an unpolarized hydrogen target. . 205

B.15 The Wein filter's rotation of an electron's spin. . . . . . . . . . . . . 206

C.1 Database tables to store the electronics configurations, polarized source settings and slow controls information (e.g. magnet current and target temperature). These tables are keyed off of the run id in the the run table. . . . . . . . . . . . . . . . . . 210

C.2 Diagram of the complete analysis portion of the database. Figs. C.3, C.4, and C.5 show expanded views of this diagram. . . . . . . . . . 213

C.3 The left-hand portion of Fig. C.2 showing the analysis table, locus, deadtime and slope tables as well as the seed tables and several detector tables. . . . . . . . . . . . . . . . . . . . . . . . . . 214

C.4 The middle portion of Fig. C.2 showing the run table and all of the tables holding detector singles and multi-hit information. Also shown are the cuts tables and monitor slopes tables. . . . . . . . . . . . . . 215

C.5 The right-portion of Fig. C.2 showing all of the monitor and calibrations tables, tables with bad octant information and several of the detector tables. . . . . . . . . . . . . . . . . . 216 


\section{Chapter 1}

\section{Physics Motivation}

\subsection{Introduction}

In 460 B.C. the Greek philosopher, Democritus, asked the question: If you break a piece of matter in half, and then break it in half again, how many breaks will you have to make before you can break it no further? Democritus believed that the breaking would end because all matter was composed of fundamental particles he called "atoms". It was not until the 19th century however, that John Dalton placed the atom on a solid foothold as a fundamental physical and chemical object.

In 1911, Ernest Rutherford published his atomic theory describing the atom as having a central positive nucleus surrounded by negative orbiting electrons. He came to this conclusion following the results of an experiment that he supervised that involved firing alpha particles through thin gold foil. The results demonstrated the existence of the atomic nucleus when a small percentage of alpha particles were scattered back from the foil while the vast majority passed through with no effect.

In 1919 Rutherford conducted another important experiment when he bombarded nitrogen gas with alpha particles where his scintillation detectors detected signatures of hydrogen nuclei. From this he posited that the hydrogen nucleus is present in other nuclei as an elementary particle, which he called the proton.

In 1964, the quark model was independently proposed by Murray Gell-Mann 
and George Zweig. In 1968, deep inelastic scattering (DIS) experiments at the Stanford Linear Accelerator Center (SLAC), an experiment similar in approach to Rutherford's, confirmed that the proton contained point-like objects and was therefore not an elementary particle. These particles were later identified as up (u) and down (d) quarks. The strange quark's existence was indirectly validated by the SLAC scattering experiments by providing an explanation for the kaons and pions discovered in cosmic rays in 1947.

Today, quantum chromodynamics (QCD) describes the strong interactions of the quarks and gluons that comprise the hadrons and is an important part of the current Standard Model for elementary particle physics. In the Standard Model, nucleons are not only comprised of their valence quarks, i.e. a proton has two up quarks and one down (uud) while a neutron has two down and one up quark $(d d u)$, but each nucleon also has virtual quark-antiquark pairs that continually emerge and annihilate in the quark-gluon sea. Sea quarks form when a gluon splits; this process also works in reverse in that the annihilation of two sea quarks produces a gluon. The result is a constant flux of gluon splits and creation colloquially known as the "sea".

The sea quarks are not restricted to the flavors of the valence quarks within each nucleon, but could theoretically consist of any of the six types of quarks with the likeliest combinations consisting of the quark pairs with the smallest masses $-u$, $d$, and strange $(s)$. Because ordinary nucleons have valence quarks with up and down flavors, any evidence of strange quarks represents quark effects solely from the quark-gluon sea. For this reason, there has been a significant experimental push to 
measure various properties of the strange quark.

The rest of this chapter summarizes the efforts to characterize the physics of strange quarks in the nucleon and provides motivation for the $G^{0}$ experiment. Chapter 2 describes the theory behind the experiment. Chapter 3 discusses similar experimental approaches and their results. Chapter 4 describes the experimental

apparatus used to collect data during the $G^{0}$ backward angle measurement and provides a brief description of the forward angle apparatus. Chapter 5 discusses the procedures used to analyze the collected data. The final results are presented in Chapter 6. Appendix A contains the details of the electromagnetic radiative correction procedures. Appendix B discusses the design and performance of the luminosity detectors, and Appendix C contains the details of the MySql database used during the $G^{0}$ backward angle experiment.

\subsection{Strangeness in the Nucleon}

A series of experimental programs have been underway since the early 1970's to measure various strange quark observables of the proton and neutron over a broad range of four-momentum transfers. The $G^{0}$ experiment was motivated in part because of experimental evidence from these programs, that strange quarks contribute to some of the properties of the nucleon, such as mass, momentum and spin. 


\subsection{Strangeness Contribution to the Nucleon's Momentum}

The NuTev (Neutrinos at the Tevatron) collaboration, using data from experimental runs in 1996-1997 at Fermi National Accelerator Laboratory, measured the strange sea contribution to the nucleon momentum with respect to the non-strange contributions to the sea. The data were from deep inelastic neutrino-nucleon scattering, which capitalizes on the fact that the charged current weak interaction between muon neutrinos and quarks were predominantly sensitive to the strange quarks. If strange quarks are present, then a reaction such as $\nu_{\mu}+s \rightarrow \mu^{-}+c$, where muon neutrinos interact with $s$ to produce a negative muon and a charm quark, $c$. The $c$ will subsequently decay semileptonically to produce a positive muon, $\mu^{+}$, resulting in dimuon $\left(\mu^{+} \mu^{-}\right)$pairs as observables. From these data they were able to measure $\kappa$, which determines the size of the strange sea relative to the non-strange sea and which can be expressed as,$\kappa \simeq 2 \bar{s} /(\bar{u}+\bar{d}))$. They found at $Q^{2}=16(\mathrm{GeV})^{2}\left[\mathrm{~A}^{+} 99 \mathrm{a}\right]$

$$
\kappa=0.42 \pm 0.07 \text { (stat) } \pm 0.06 \text { (sys) }
$$

where $Q^{2}$ is the four-momentum transfer. This is a clear indication that strange quarks are present in a nucleon's sea, although it is difficult to associate ordinary observables with this momentum fraction from the parton model.

\subsubsection{Strangeness Contribution to the Nucleon Mass}

There is evidence of the strange quark contribution to the mass of the nucleon from the studies of the $\pi \mathrm{N} \sigma$-term [GLS91]. The mass of the nucleon is given by the matrix element $M_{N}=\left\langle N\left|H_{Q C D}\right| N\right\rangle$ where $H_{Q C D}$ is the Hamiltonian of a nucleon 
and $\langle N|(|N\rangle)$ is the initial (final) state of the nucleon. An empirical measure of the chiral asymmetry generated by the $u$ and $d$ quark masses in QCD is the $\pi N \sigma$-term. It is expressed as [GL85]

$$
\sigma=\frac{\hat{m}}{2 m}\langle N|\bar{u} u+\bar{d} d| N\rangle
$$

where $\hat{m}=\left(m_{u}+m_{d}\right) / 2$, and $m$ is the proton mass. Algebraically, $\sigma$ can be written as [Sai94]

$$
\sigma=\frac{\hat{m}}{2 m} \frac{\langle N|\bar{u} u+\bar{d} d-2 \bar{s} s| N\rangle}{1-y}
$$

where $y$ represents the strange quark content of the proton, defined as

$$
y=\frac{2\langle N|\bar{s} s| N\rangle}{\langle N|\bar{u} u+\bar{d} d| N\rangle} .
$$

Chiral perturbation theory (ChPT) allows for the determination of the combination [Sai94]

$$
\hat{\sigma}=\sigma(1-y)
$$

from the baryon spectrum. If the sigma term can be measured, the strangeness content, $y$, can be estimated.

The $\pi N$ scattering amplitude at the unphysical Cheng-Dashen point $\left(Q^{2}=\right.$ $\left.2 m_{\pi}^{2}\right)$ [CD71], can be related to $\sigma$ using chiral symmetry as,

$$
\Sigma_{\pi N}=F_{\pi}^{2} \bar{D}^{+}\left(s=M_{N}^{2}, Q^{2}=2 m_{\pi}^{2}\right) \simeq \hat{\sigma}\left(Q^{2}=2 m_{\pi}^{2}\right)
$$

where $F_{\pi}$ is the pion decay constant, $\bar{D}^{+}$is the $\pi$-N scattering amplitude, and $s$ is the invariant mass. Because the Cheng-Dashen point is unphysical, the experimental data have to be extrapolated to that momentum transfer. Calculating the mass term requires extrapolating the data down to zero momentum transfer, which is 
non-trivial and requires calculation of higher order terms. Gasser and Leutwyler [GL85] calculated a value of $\hat{\sigma} \sim 45 \mathrm{MeV}$.

Hyperon mass splitting can also be used to constrain the scalar matrix elements. At leading order,

$$
\frac{1}{3}\left(\hat{m}-m_{s}\right)\langle N|\bar{u} u+\bar{d} d-2 \bar{s} s| N\rangle=M_{\Lambda}-M_{\Xi}
$$

where $M_{\Lambda}$ and $M_{\Xi}$ are hyperon masses. Including higher order chiral corrections yields a value of $\hat{\sigma} \sim 35 \mathrm{MeV}$.

If there is no strangeness contribution to the nucleon mass, then $\Sigma_{\pi N}=\hat{\sigma}=\sigma$. The $\sim 10 \mathrm{MeV}$ difference between the two values of $\hat{\sigma}$ yields $y \sim 0.2$. Gasser, Leutwyler, and Sainio [GLS91] suggested that the discrepancy may be due to a contribution from $\bar{s} s$ to the nucleon mass of the order of $\sigma_{s}=m_{s}\langle N|\bar{s} s| N\rangle \sim 130$ $\mathrm{MeV}$. This result is not conclusive as other analyses have been performed with results for $m_{s}$ ranging from $-150 \mathrm{MeV}$ to $250 \mathrm{MeV}$ [Sai02].

\subsubsection{Strangeness and Nucleon Spin}

For a polarized nucleon, $q_{i}^{+(-)}(x)$ represents the number of quarks, of flavor $i$ $(u, \bar{u}, d, \bar{d}, s$, and $\bar{s})$ polarized in the same (opposite) direction as the nucleon and as before, $x$ is the fraction of the proton's momentum carried by the struck quark in the infinite momentum frame. The total contribution of quark with flavor $i$ to the spin of the nucleon is

$$
\Delta q_{i} \int_{0}^{1}\left[q_{i}^{+}(x)-q_{i}^{-}(x)\right] d x
$$


which is also called the axial charge because it is related to the matrix element of the axial current in the nucleon state.

In the quark-parton model, the spin-dependent structure function $g_{1}(x)$ is related to the quark spin distributions via [FJ01]

$$
g_{1}(x)=\frac{1}{2} \sum_{i} e_{q_{i}}^{2} \Delta q_{i}(x) .
$$

There have been several measurements of spin asymmetry in DIS of longitudinally polarized muons by longitudinally polarized nucleons at both SLAC and CERN where $g_{1}(x)$ was extracted from the asymmetry measurement. The initial interest in measuring $g_{1}$ was to compare the measurements to the Ellis-Jaffe and Bjorken sum rules.

The Ellis-Jaffe sum rule ignores the three heavy quark flavors and assumes the strange quark and sea polarizations are zero. For the proton, the Ellis-Jaffe sum rule gives [FJ01]:

$$
\Gamma_{1}=\int_{0}^{1} g_{1}^{p}(x) d x=0.186 \pm 0.004
$$

While the Ellis-Jaffe assumptions may be violated, the Bjorken sum rule [Bjo66, Bjo70] requires only isospin symmetry (eg. $\left.\Delta u^{p}=\Delta d^{n}\right)$. For the proton, the Bjorken sum rule gives [FJ01]

$$
\Gamma_{1}=\int_{0}^{1} g_{1}^{p}(x) d x=0.211 \pm 0.001
$$

Comparisons of these predictions with experiment requires forming the integrals of $g_{1}$ over the full $x$ ranges, requiring extrapolations to include regions of unmeasured $x$. The E155 collaboration reported results $\left[\mathrm{A}^{+} 06 \mathrm{~d}\right]$ from a global analysis 
of spin structure function integrals. Those results are compared with a calculation of the sum rules by Larin [LV91] as shown in Table 1.1. Table 1.1 shows experimental verification of the Bjorken sum rule.

Table 1.1: Comparison of Sum Rule predictions including higher order corrections [LV91] with a global analysis of E155 results [ $A^{+}$06d] for $Q^{2}=5(\mathrm{GeV} / \mathrm{c})^{2}$ [FJ01].

\begin{tabular}{|c|c|c|}
\hline Sum Rule & Calculation & Experiment \\
\hline EJ Sum $\Gamma_{1}^{p}$ & $0.163 \pm 0.004$ & $0.118 \pm 0.008$ \\
EJ Sum $\Gamma_{1}^{n}$ & $-0.019 \pm 0.004$ & $-0.058 \pm 0.009$ \\
Bj Sum $\Gamma_{1}^{p}-\Gamma_{1}^{n}$ & $0.181 \pm 0.005$ & $0.176 \pm 0.008$ \\
\hline
\end{tabular}

Using the experimental values shown in Table 1.1 the values of the individual quark contributions can be calculated to leading order. This gives [FJ01]

$$
\Delta s+\Delta \bar{s}=-0.14 \pm 0.03
$$

These measurements indicate that the overall contribution of quarks to the nucleon spin is quite small and that $\Delta s$ is small and negative. Because the total spin from the quarks is much smaller than the total spin of the nucleon, this "spin crisis" has prompted extensive study of the spin-flavor structure of the nucleon using polarized DIS. The small and negative values for $\Delta s$ have encouraged further study of strange quark contributions to the nucleon.

\subsubsection{Vector Strange Quark Matrix Elements}

In 1988, Kaplan and Manohar [KM88] suggested that there was evidence for non-zero scalar and axial-vector strange quark matrix elements in the nucleon and that neutral current experiments, such as $\nu-N$ scattering, could provide a means 
to access this information. In 1989, McKeown [McK89] and Beck [Bec89] published papers that described an experimental program that utilized parity-violating electron scattering to access the weak neutral current sector of the proton in order to extract information about the strange quark vector matrix elements. The experiment that is the topic of this thesis describes one such program, the $G^{0}$ experiment, and specifically, the backward angle $G^{0}$ measurement, that was conducted in Hall C at Thomas Jefferson National Accelerator Facility (JLab). 


\section{Chapter 2}

\section{Theory and Formalism}

Elastic (quasi-elastic) electron scattering from a proton (nucleon) can be described to first order as a single photon $(\gamma)$ exchange for the electromagnetic interaction and single vector boson $(\mathrm{Z})$ exchange for the neutral weak interaction, as shown in the Feynman diagrams in Fig. 2.1.
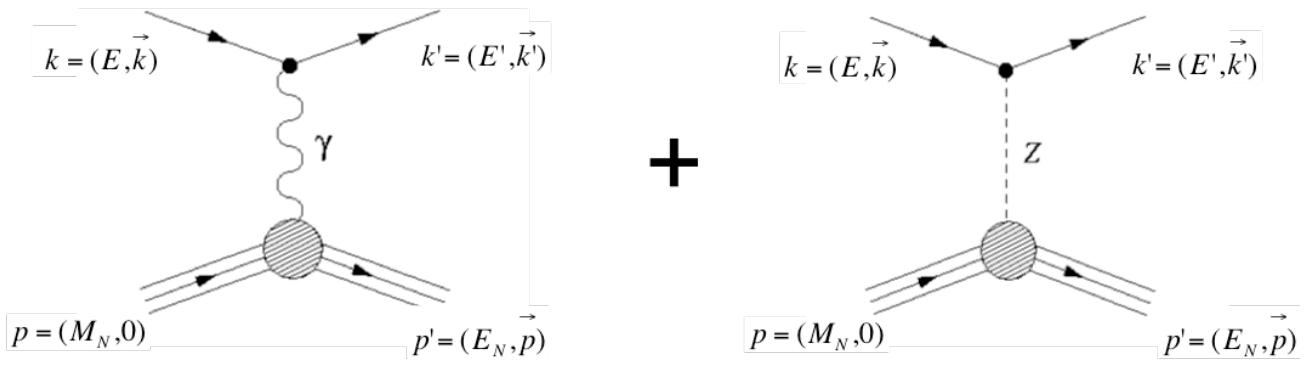

Figure 2.1: Total leading order amplitude for electron scattering from a nucleon is the sum of the leading order electromagnetic and neutral current amplitudes.

The incident electron, $e$, described by the four-vector $k=(E, \vec{k})$ scatters from a target nucleon, $p=\left(M_{N}, 0\right)$. After the scattering event, the electron is described as $k^{\prime}=\left(E^{\prime}, \overrightarrow{k^{\prime}}\right)$ and the nucleon as $p^{\prime}=\left(E_{N}, \overrightarrow{p^{\prime}}\right)$. The four vector representing the energy and momentum lost by the electron is $Q=(\omega, \vec{q})$ where $\omega=E-E^{\prime}$ and $\vec{q}=\vec{k}-\overrightarrow{k^{\prime}}$. The electron is treated in the extreme relativistic limit, so that $m_{e}^{2}=0 . Q^{2}$ is the invariant four-momentum transfer of the scattering and is defined as $Q^{2}=-q^{2}=-\left(\omega^{2}-\vec{q}^{2}\right)$. For elastic electron scattering, $Q^{2}=4 E E^{\prime} \sin ^{2}\left(\frac{\theta_{e}}{2}\right)$ where $\theta_{e}$ is the electron scattering angle in the laboratory frame of reference. 
Each Feynman diagram has an associated invariant amplitude, $\mathcal{M}^{E M}$ or $\mathcal{M}^{N C}$. The amplitudes are summed to form the total first order invariant amplitude for the interaction, $\mathcal{M}$. The dominant amplitude is the electromagnetic interaction while the neutral weak interaction generates a small amplitude that is detectable via quantum interference. Because the weak interaction violates parity, the interference effects imply the existence of small pseudoscalar observables in electron scattering [BM01].

\subsection{Nucleon Form Factors}

The amplitude for the electromagnetic current (EC), following the notation of $\left[\mathrm{M}^{+} 94\right]$, can be written $\mathrm{as}^{1}$

$$
\mathcal{M}^{E M}=\frac{4 \pi \alpha}{q^{2}} Q_{l} l^{\mu} J_{\mu}^{E M}
$$

and the amplitude for the weak neutral current (NC) can be expressed as

$$
\mathcal{M}^{N C}=-\frac{G_{F}}{2 \sqrt{2}}\left(g_{V}^{l} l^{\mu}+g_{A}^{l} l^{\mu 5}\right)\left(J_{\mu}^{N C}+J_{\mu 5}^{N C}\right)
$$

where $Q_{l}, g_{V}^{l}$, and $g_{A}^{l}$ are the lepton electromagnetic, vector, and axial-vector charges respectively (Table 2.1). $\mathcal{M}^{N C}$ shows no $q^{2}$ dependence because when $q^{2}<<M_{N C}$, the weak interaction is usually treated as a contact interaction with a strength determined by the Fermi constant [BPS05], $G_{F} \approx 1.166367(5) \times 10^{-5} \mathrm{GeV}^{-2}$ [AoPDG08]. The lepton vector and axial-vector currents, $l^{\mu}$ and $l^{\mu 5}$ respectively, can be expressed

\footnotetext{
${ }^{1}$ One point of departure from the formalism of $\left[\mathrm{M}^{+} 94\right]$ where $Q \equiv k-k^{\prime}$. In this work $Q^{2} \equiv$ $-q^{2}>0$.
} 
as

$$
\begin{aligned}
l^{\mu} & =\bar{u}_{l} \gamma^{\mu} u_{l}, \\
l^{\mu 5} & =\bar{u}_{l} \gamma^{\mu} \gamma^{5} u_{l},
\end{aligned}
$$

with $\mu_{l}$ representing the four-component lepton spinor, $\gamma^{\mu}(\mu=0,1,2,3)$ defining the Dirac matrices, with $\gamma^{5}=i \gamma^{0} \gamma^{1} \gamma^{2} \gamma^{3}$. The fine structure constant is $\alpha=\frac{e^{2}}{4 \pi}$ where $e$ is the coupling strength of the electromagnetic interaction. ${ }^{2}$ The Fermi constant [BM01] can be expressed as

$$
G_{F}=\frac{e^{2}}{4 \sqrt{2} M_{W}^{2} \sin ^{2} \theta_{W}},
$$

where $M_{W}$ is the mass of the $W$ boson and $\theta_{W}$ is the weak mixing angle. The weak mixing angle can be related to the neutral and charged boson masses by the relation

$$
\sin ^{2} \theta_{W} \equiv 1-\frac{M_{W}^{2}}{M_{Z}^{2}}
$$

Table 2.1: Electroweak charges of elementary fermions $\left[\mathrm{M}^{+} 94\right]$

\begin{tabular}{|c|c|c|c|}
\hline Fermion & $Q_{l}$ & $g_{V}^{l}$ & $g_{A}^{l}$ \\
\hline \hline$\nu_{e}, \nu_{\mu}, \nu_{\tau}$ & 0 & 1 & -1 \\
$e^{-}, \mu^{-}, \tau^{-}$ & -1 & $-1+4 \sin ^{2} \theta_{W}$ & 1 \\
$u, c, t$ & $\frac{2}{3}$ & $1-\frac{8}{3} \sin ^{2} \theta_{W}$ & -1 \\
$d, s, b$ & $-\frac{1}{3}$ & $-1+\frac{4}{3} \sin ^{2} \theta_{W}$ & 1 \\
\hline
\end{tabular}

Because the nucleon has internal structure, the hadronic currents include a set of form factors defined to encompass this complicated structure. Assuming gauge

\footnotetext{
${ }^{2}$ Unless otherwise noted, the equations in this work have been derived in a set of units where $\hbar c=1$.
} 
and Lorentz invariance, the hadronic vector currents of both the electromagnetic and weak interactions (one boson exchange) can be expressed as [HM84]

$$
J_{\mu}^{j}=\bar{U}_{N}\left[F_{1}^{j}\left(q^{2}\right) \gamma^{\mu}+F_{2}^{j}\left(q^{2}\right) \frac{i \sigma^{\mu \nu} q_{\nu}}{2 M}\right] U_{N},
$$

where $U_{N}$ is a nucleon spinor, $j$ denotes the type of interaction $(\gamma / \mathrm{EM}$ or $Z / \mathrm{NC})$, $\sigma^{\mu \nu}=\frac{i}{2}\left[\gamma^{\mu}, \gamma^{\nu}\right]$, and $N$ is the nucleon $(p$ or $n)$. The form factors $F_{1}^{j}$ and $F_{2}^{j}$ are the Dirac and Pauli form factors, respectively. They are normalized such that for the electromagnetic interaction, when $Q^{2}=0$,

$$
\begin{aligned}
& F_{1}^{\gamma}(0)=Q_{N}, \\
& F_{2}^{\gamma}(0)=\kappa_{N},
\end{aligned}
$$

where $Q_{N}$ is the electric charge of the nucleon (in units of $e$ ), and $\kappa_{N}$ is the anomalous magnetic moment of the nucleon (in units of the Bohr magneton).

There is an additional hadronic current to the neutral weak interaction due to the axial vector component. When this is included with the neutral weak vector current, the total hadronic current for the neutral weak interaction can be represented as

$$
J_{\mu}^{N C}=\bar{U}_{N}\left[F_{1}^{Z}\left(q^{2}\right) \gamma^{\mu}+F_{2}^{Z}\left(q^{2}\right) \frac{i \sigma^{\mu \nu} q_{\nu}}{2 M}+G_{A}^{e N} \gamma^{\mu} \gamma_{5}\right] U_{N}
$$

where $G_{A}^{e N}$ is the nucleon's neutral weak axial form factor.

In practice, it is frequently better to use a linear combination of $F_{1,2}$, known as the Sachs form factors expressed as [Sac62]

$$
\begin{aligned}
& G_{E}^{j N}=F_{1}^{j N}-\tau F_{2}^{j N}, \\
& G_{M}^{j N}=F_{1}^{j N}+F_{2}^{j N},
\end{aligned}
$$


where $\tau=\frac{Q^{2}}{4 M^{2}}$ and $j$ is the interaction type, either $\gamma$ for electromagnetic or $Z$ for neutral weak. At $Q^{2}=0, G_{E}^{\gamma N}$ and $G_{M}^{\gamma N}$ are equivalent to the electric charge and magnetic moment of the nucleon, respectively. Additionally, in the Breit frame, or the center of mass frame defined by $\vec{p}^{\prime}=-\vec{p}$, the Sachs form factors are the Fourier transforms of the nucleon charge and magnetic moment distributions [Sac62].

\subsubsection{Flavor Decomposition}

The hadronic currents, $J_{\mu}^{E M}, J_{\mu}^{N C}$, and $J_{\mu 5}^{N C}$, can also be expressed as

$$
J_{\mu}^{i} \equiv\left\langle H\left|\hat{J}_{\mu}^{i}\right| H\right\rangle
$$

where $|H\rangle$ is any hadronic state, which in this case is either a proton or a neutron. Assuming a point-like interaction between the gauge bosons $(\gamma, Z)$ and the quarks internal to the nucleon, the quark current operators can be written as $\left[\mathrm{M}^{+} 94\right]$ :

$$
\begin{aligned}
\hat{J}_{\mu}^{E M} & \equiv \sum_{q} Q_{q} \bar{u}_{q} \gamma_{\mu} u_{q} \\
\hat{J}_{\mu}^{N C} & \equiv \sum_{q} g_{V}^{q} \bar{u}_{q} \gamma_{\mu} u_{q} \\
\hat{J}_{\mu 5}^{N C} & \equiv \sum_{q} g_{A}^{q} \bar{u}_{q} \gamma_{\mu} \gamma_{5} u_{q}
\end{aligned}
$$

where the summation is over all quark flavors, which implicitly includes both quarks and their anti-quarks. The values of $Q_{q}, g_{V}^{q}$, and $g_{A}^{q}$ are given in Table 2.1. Expressing the quark current operators in this manner allows us to express the hadronic currents as

$$
\begin{aligned}
J_{\mu}^{E M} & \equiv \bar{U}_{N} \sum_{q} Q_{q}\left[F_{1}^{q} \gamma^{\mu}+F_{2}^{q} \frac{i \sigma^{\mu \nu} q_{\nu}}{2 M}\right] U_{N} \\
J_{\mu}^{N C} & \equiv \bar{U}_{N} \sum_{q}\left[g_{v}^{q}\left(F_{1}^{q} \gamma^{\mu}+F_{2}^{q} \frac{i \sigma^{\mu \nu} q_{\nu}}{2 M}\right)+g_{A}^{q} G_{A}^{q} \gamma^{\mu} \gamma_{5}\right] U_{N}
\end{aligned}
$$


where $F_{1}^{q}, F_{2}^{q}$, and $G_{A}^{q}$, are the Dirac, Pauli, and axial form factors, respectively, with quark flavor $q$. The quark form factors, $F_{1}^{q}$ and $F_{2}^{q}$, are interaction independent and are the same in eqns. 2.17 and 2.18. Comparing eqns. 2.7 and 2.10 with eqns. 2.17 and 2.18 it is evident that the nucleon form factors $F_{1,2}^{\gamma N}, F_{1,2}^{Z N}$, and $G_{A}^{e N}$ can be expressed in terms of the quark flavor form factors as

$$
\begin{aligned}
F_{1,2}^{\gamma N} & =\sum_{q} Q_{q} F_{1,2}^{q}, \\
F_{1,2}^{Z N} & =\sum_{q} g_{v}^{q} F_{1,2}^{q}, \\
G_{A}^{e N} & =\sum_{q} g_{A}^{q} G_{A}^{q},
\end{aligned}
$$

where $Q_{q}, g_{v}^{q}$, and $g_{A}^{q}$ are given in Table 2.1. This results in a set of five nucleon form factors in terms of 12 unknown quark form factors for each nucleon. The electromagnetic and neutral weak Sachs form factors can also easily be expressed as quark flavor form factors

$$
\begin{aligned}
G_{E, M}^{\gamma N} & =\sum_{q} Q_{q} G_{E, M}^{q}, \\
G_{E, M}^{Z N} & =\sum_{q} g_{v}^{q} G_{E, M}^{q} .
\end{aligned}
$$

\subsubsection{Flavor Vector Form Factors}

Because the masses of the three heaviest quarks $(c, b$, and $t)$ are greater than the mass of the proton, there is a strong suppression of their contributions to the properties of nucleons. This allows us to write the Sachs form factors in terms of 
the three lightest quark flavors

$$
\begin{aligned}
G_{E, M}^{\gamma N} & =\frac{2}{3} G_{E, M}^{u N}-\frac{1}{3}\left(G_{E, M}^{d N}+G_{E, M}^{s N}\right) \\
G_{E, M}^{Z N} & =\left(1-\frac{8}{3} \sin ^{2} \theta_{W}\right) G_{E, M}^{u N}-\left(1-\frac{4}{3} \sin ^{2} \theta_{W}\right)\left(G_{E, M}^{d N}+G_{E, M}^{s N}\right) .
\end{aligned}
$$

The number of unknowns can be further reduced by assuming charge symmetry which capitalizes on the fact that the wave functions of the $u$ and $d$ quarks in the proton are the same as the $d$ and $u$ quarks in the neutron [Mil98]. In addition, it is generally assumed that the strange quark distributions in the proton and the neutron are the same. These assumptions allow the following result.

$$
G_{E, M}^{u, p}=G_{E, M}^{d, n} \equiv G_{E, M}^{u}, \quad G_{E, M}^{d, p}=G_{E, M}^{u, n} \equiv G_{E, M}^{d}, \quad G_{E, M}^{s, p}=G_{E, M}^{s, n}=G_{E, M}^{s}
$$

which reduces the number of unknowns and simplifies the notation. Charge symmetry breaking occurs due to the differing masses and charges of the $u$ and $d$ quarks, but this effect is generally less than $1 \%$ of the electromagnetic form factors [DP95], [Mil98], and [LM99].

Explicitly writing the proton and neutron Sachs vector form factors using Eqns. (2.20 and 2.21) and charge symmetry yields

$$
\begin{aligned}
G_{E, M}^{\gamma p} & =\frac{2}{3} G_{E, M}^{u}-\frac{1}{3}\left(G_{E, M}^{d}+G_{E, M}^{s}\right) \\
G_{E, M}^{\gamma n} & =\frac{2}{3} G_{E, M}^{d}-\frac{1}{3}\left(G_{E, M}^{u}+G_{E, M}^{s}\right) \\
G_{E, M}^{Z p} & =\left(1-\frac{8}{3} \sin ^{2} \theta_{W}\right) G_{E, M}^{u}-\left(1-\frac{4}{3} \sin ^{2} \theta_{W}\right)\left(G_{E, M}^{d}+G_{E, M}^{s}\right) \\
G_{E, M}^{Z n} & =\left(1-\frac{8}{3} \sin ^{2} \theta_{W}\right) G_{E, M}^{d}-\left(1-\frac{4}{3} \sin ^{2} \theta_{W}\right)\left(G_{E, M}^{u}+G_{E, M}^{s}\right)
\end{aligned}
$$

Rearranging Eqns. 2.27, 2.28, and 2.29, it is possible to express the proton's neutral 
weak form factor as

$$
G_{E, M}^{Z p}=\left(1-4 \sin ^{2} \theta_{W}\right) G_{E, M}^{\gamma p}-G_{E, M}^{\gamma n}-G_{E, M}^{s}
$$

This important results shows that a measurement of the neutral weak form factors, when combined with the well-known values for the electromagnetic form factors, provides a determination of the vector strange form factors.

\subsubsection{Flavor Axial Form Factors}

The neutral weak axial form factors can also be expressed as a sum of the individual quark flavor form factors, weighted by the weak axial charge of that flavor

$$
G_{A}^{Z N}=g_{A}^{u} G_{A}^{u N}+g_{A}^{d} G_{A}^{d N}+g_{A}^{s} G_{A}^{s N}
$$

Assuming charge symmetry as well as the same strange quark distributions in protons and neutrons results in:

$$
G_{A}^{u p}=G_{A}^{d n} \equiv G_{A}^{u}, \quad G_{A}^{d p}=G_{A}^{u n} \equiv G_{A}^{d}, \quad G_{A}^{s N} \equiv G_{A}^{s},
$$

and using the values in Table 2.1, the neutral weak axial form factors can be expressed in the following simplified manner

$$
\begin{aligned}
G_{A}^{Z p} & =-\left(G_{A}^{u}-G_{A}^{d}\right)+G_{A}^{s} \\
G_{A}^{Z n} & =\left(G_{A}^{u}-G_{A}^{d}\right)+G_{A}^{s} .
\end{aligned}
$$

In the limit of "no strangeness", the axial form factor has an explicit isovector structure:

$$
G_{A}^{Z}=-\left(G_{A}^{u}-G_{A}^{d}\right)=-\tau_{3} G_{A}
$$


where $\tau_{3}=+1(-1)$ for a proton (neutron). In the lowest order limit of single $Z$-boson exchange, the isovector and $\mathrm{SU}(3)$ singlet contributions survive:

$$
G_{A}^{Z}=-\tau_{3} G_{A}+G_{A}^{s}
$$

where $G_{A}^{s}$ is the strange quark contribution to nucleon spin. $G_{A}^{s}$ comes from the axial vector strange matrix element $\left\langle p\left|\bar{s} \gamma_{\mu} \gamma_{5} s\right| p\right\rangle$ measured in deep inelastic scattering experiments and discussed in section 2.3. Higher order corrections to $G_{A}^{s}$ are expected to be significant and are also addressed in section 2.3.

\subsubsection{Flavor Singlet Form Factors}

The flavor decomposition may also be made in terms of the SU(3) flavor basis where the electromagnetic form factors are written as a sum of isovector and octet terms,

$$
G_{E, M}^{\gamma p}=G_{E, M}^{3, p}+\frac{1}{\sqrt{3}} G_{E, M}^{8, p}
$$

The isovector form factor $G_{E, M}^{3, p}$ and the octet form factor, $G_{E, M}^{8, p}$ can be written in terms of individual quark contributions,

$$
\begin{aligned}
G_{E, M}^{3, p} & =\frac{1}{2}\left(G_{E, M}^{u}-G_{E, M}^{d}\right) \\
G_{E, M}^{8, p} & =\frac{1}{2 \sqrt{3}}\left(G_{E, M}^{u}+G_{E, M}^{d}-2 G_{E, M}^{s}\right) .
\end{aligned}
$$

In this basis, the neutral weak form factors are

$$
\begin{aligned}
G_{E, M}^{Z p} & =\left(\frac{1}{2}-\sin ^{2} \theta_{w}\right) G_{E, M}^{3, p}+\left(\frac{1}{2 \sqrt{3}}-\frac{1}{\sqrt{3}} \sin ^{2} \theta_{w}\right) G_{E, M}^{8, p}-\frac{1}{4} G_{E, M}^{0},(2.4 \\
G_{E, M}^{Z p} & =\left(\frac{1}{2}-\sin ^{2} \theta_{w}\right) G_{E, M}^{\gamma, p}-\frac{1}{4} G_{E, M}^{0}
\end{aligned}
$$


where

$$
G_{E, M}^{0 p}=\frac{1}{3}\left(G_{E, M}^{u}+G_{E, M}^{d}+G_{E, M}^{s}\right)
$$

is the flavor singlet form factor. A measurement of $G_{E, M}^{Z p}$, as taken during the $G^{0}$ experiment, when combined with the known values for the proton's electromagnetic form factors, determines the flavor singlet form factor, $G_{E, M}^{0 p}$. This is the origin of the name of the $G^{0}$ experiment.

\subsection{Parity Violation in Electron Scattering}

After McKeown [McK89] and Beck [Bec89] explained how $G_{E, M}^{Z}$ could be measured using parity-violating electron scattering, a series of experimental programs were funded and initiated. These experiments include the $G^{0}$ Backward Angle measurement at Jefferson Laboratory's Continuous Electron Beam Facility (CEBAF), which is the topic of this thesis, and several others that are described and discussed in Chap. 3.

\subsubsection{Experimental Observables: Neutral Weak Vector Form Factors}

As previously mentioned, the total invariant amplitude for e- $\mathrm{N}$ elastic or quasielastic scattering is a coherent sum of the electromagnetic and neutral current amplitudes

$$
\mathcal{M}=\mathcal{M}^{E M}+\mathcal{M}^{N C}
$$

where leading order values of $\mathcal{M}^{E M}$ and $\mathcal{M}^{N C}$ are given in Eqns. 2.1 and 2.2 respectively. The scattering probability, $d \sigma$, is proportional to the total invariant 
amplitude squared

$$
d \sigma=\propto|\mathcal{M}|^{2}=\propto\left|\mathcal{M}^{E M}\right|^{2}+2 \mathcal{M}^{E M^{*}} \mathcal{M}^{N C}+\left|\mathcal{M}_{N C}\right|^{2}
$$

where $\mathcal{M}^{E M^{*}}$ represents the complex conjugate of $\mathcal{M}^{E M}$. The neutral weak amplitude is strongly suppressed relative to the electromagnetic amplitude in an absolute cross section measurement. Therefore, in order to measure the neutral weak vector form factors, it is necessary to take advantage of the parity-violating nature of the weak interaction. Because the weak interaction violates parity while the electromagnetic interaction does not, it is the interference term, $2 \mathcal{M}^{E M^{*}} \mathcal{M}^{N C}$, that is the cause of the parity violation seen in e-N elastic and quasi-elastic scattering.

Operators formed from a vector and an axial vector operator are parityviolating while operators formed from squares of either conserve parity. The parityviolating component of the neutral current amplitude arises from the cross terms of the axial and vector currents. The amplitude can be written as a sum of parityconserving $\mathcal{M}_{P C}^{N C}$ and parity-violating $\mathcal{M}_{P V}^{N C}$ amplitudes:

$$
\begin{aligned}
\mathcal{M}_{P C} & =-\frac{G_{F}}{2 \sqrt{2}}\left(g_{V}^{l} l^{\mu} J_{\mu}^{N C}+g_{A}^{l} l^{\mu 5} J_{\mu 5}^{N C}\right), \\
\mathcal{M}_{P V} & =-\frac{G_{F}}{2 \sqrt{2}}\left(g_{V}^{l} l^{\mu 5} J_{\mu}^{N C}+g_{A}^{l} l^{\mu} J_{\mu 5}^{N C}\right) .
\end{aligned}
$$

Parity violation can be probed using longitudinally polarized electrons where the two states of electron polarization (parallel or anti-parallel to the beam direction) correspond to the two parity states. The parity-violating asymmetry for the scattering of the polarized electrons from a target of unpolarized protons is defined 
as the difference in the cross section for each helicity state, divided by the sum of the cross sections:

$$
A \equiv \frac{\sigma_{+}-\sigma-}{\sigma_{+}+\sigma-}
$$

Eliminating the parity conserving terms, and any terms with $G_{F}^{2}$ in the numerator or $G_{F}$ in the denominator, provides the following expression for the parityviolating asymmetry:

$$
A \approx 2 \frac{\mathcal{M}^{E M^{*}} \mathcal{M}^{P V}}{\left|\mathcal{M}^{E M}\right|^{2}}
$$

Substituting in Eqns. 2.1, 2.7, 2.10, 2.11, 2.12, and 2.47 and rearranging the terms as in Refs. [RS74] and [BPS05] yields:

$$
A=-\frac{G_{F} Q^{2}}{4 \pi \alpha \sqrt{2}} \frac{A_{E}+A_{M}+A_{A}}{\left[\epsilon\left(G_{M}^{\gamma}\right)^{2}+\tau\left(G_{M}^{\gamma}\right)^{2}\right]}
$$

with the electric, magnetic, and axial components of the asymmetry expressed as;

$$
\begin{aligned}
& A_{E}=\epsilon G_{E}^{Z}\left(Q^{2}\right) G_{E}^{\gamma}\left(Q^{2}\right) \\
& A_{M}=\tau G_{M}^{Z}\left(Q^{2}\right) G_{M}^{\gamma}\left(Q^{2}\right) \\
& A_{A}=-\left(1-4 \sin ^{2} \theta_{W}\right) \sqrt{\tau(1+\tau)\left(1-\epsilon^{2}\right)} G_{A}^{e}\left(Q^{2}\right) G_{M}^{\gamma}\left(Q^{2}\right)
\end{aligned}
$$

and

$$
\tau=\frac{Q^{2}}{4 M_{N}^{2}} \quad \text { and } \quad \epsilon=\frac{1}{1+2(1+\tau) \tan ^{2} \frac{\theta}{2}}
$$

Writing the asymmetry expression in the above manner clearly shows the $\gamma-Z$ interference and the sensitivity of the electric, magnetic, and axial form factors to the kinematics of the experiment. In general, forward angle experiments are sensitive to a combination of $A_{E}$ and $A_{M}$ and backward angle experiments to a combination 
of $A_{M}$ and $A_{A}$. Additionally, quasielastic scattering from an isospin 0 target, such as a deuteron can be used to enhance $A_{A}$ [BPS05].

The notation for the axial form factor has been modified here from $G_{A}^{Z}$ to $G_{A}^{e}$ in order to distinguish the form factor as seen by electron scattering from that seen by neutrino scattering where the higher order diagrams involving electromagnetic interactions are absent. This will be discussed in more detail in section 2.3.

\subsubsection{Electroweak Radiative Corrections to the Neutral Current}

The neutral weak vector and axial vector form factors derived at leading order in Section 2.1.1.1 require corrections due to higher order electroweak processes. These corrections modify the coupling constants at the interaction vertex and in effect, modify the weak vector and axial charges. The corrections fall in one of three categories: one quark, many-quark, and heavy quark renormalization. One quark radiative corrections that do not require knowledge of quark interactions can be calculated using Standard Model electroweak theory with small associated uncertainties. The electroweak calculations require a renormalization scheme to be selected, and in this work, the "MS-bar" $(\overline{M S})$ or modified minimal subtraction scheme is used $\left[\mathrm{M}^{+} 94\right]$. The weak mixing angle in this scheme is no longer defined as it was in Eqn. 2.6, but now carries a dependence on a renormalization mass scale, which in this case is the mass of the $Z$ boson, $M_{Z}=91.1876 \pm 0.0021 \mathrm{GeV}$ [AoPDG08].

For the vector weak form factors at low momentum transfers, the one quark 
corrections have a weak dependence on $Q^{2}$ and have the same $\left(1-4 \sin ^{2} \theta_{W}\right)$ multiplier as the tree-level amplitudes and therefore, despite being the dominant higherorder vector correction, are typically very small [BPS05]. One-quark axial corrections are also calculable with small errors, but unlike the vector correction, it is substantial compared to the tree-level amplitudes. The biggest effect of electroweak radiative corrections however, is the many-quark correction to the axial term.

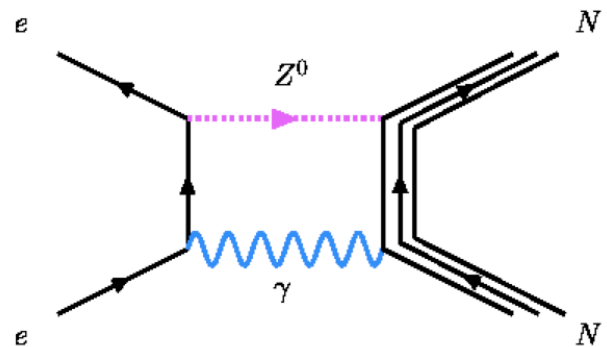

(a) $\gamma-Z$ mixing diagram

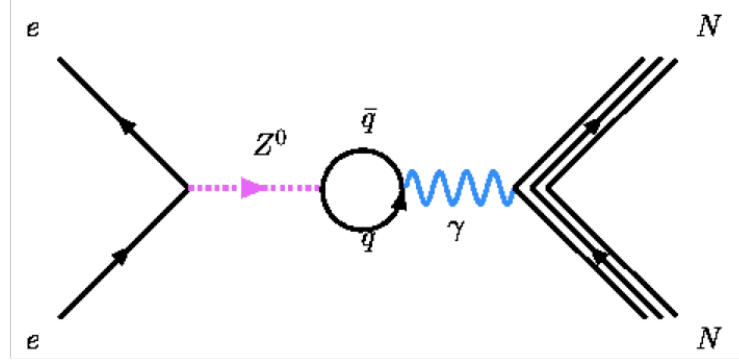

(a) $\gamma-Z$ box diagram

Figure 2.2: Representative one-quark Feynman diagrams contributing to electroweak radiative corrections.

Corrections involving many-quarks where strong interactions are included, can not be easily calculated because Perturbative Quantum Chromodynamics (PQCD) is not effective in the energy range of the $G^{0}$ measurements, $\approx 1 \mathrm{GeV}$. These corrections have a large associated uncertainty because of our inability to include all virtual hadronic states in the calculation.

The third correction is heavy quark renormalization. When the neutral weak form factors were decomposed into quark form factors (Eqns. 2.27, 2.28, 2.29, and 2.30) only the three lightest quark flavors were included. Heavy-quark renormalization of the light-quark current operators results in corrections to the neutral current couplings. These corrections were calculated by Kaplan and Manohar [KM88] and 
were found to be small, with the vector term on the order of $10^{-4}$ and $10^{-2}$ for the axial term. These corrections are neglected in this work due to their small size.

Electroweak radiative effects can be parametrized in terms of the parameters $\rho$ and $\kappa$ as proposed in the pioneering work of Marciano and Sirlin, [MS84]. In this approach, the proton's weak charge becomes

$$
Q_{w}=1-4 \sin ^{2} \theta_{W} \rightarrow \rho\left(1-4 \kappa \sin _{\theta_{W}}^{2}\right)
$$

Using this parameterization, the proton asymmetry can be written as a sum of vector, strange vector, and axial vector contributions [TBM09],

$$
A_{p}=-\left(\frac{G_{F} Q^{2}}{4 \sqrt{2} \pi \alpha}\right)\left(A_{V}+A_{s}+A_{A}\right)
$$

where

$$
\begin{aligned}
& A_{V}=\rho\left[\left(1-4 \kappa \sin _{\theta_{W}}^{2}\right)-\frac{\epsilon G_{E}^{\gamma, p} G_{E}^{\gamma, n}+\tau G_{M}^{\gamma, p} G_{M}^{\gamma, n}}{\sigma}\right], \\
& A_{s}=-\rho \frac{\epsilon G_{E}^{\gamma, p} G_{E}^{s}+\tau G_{M}^{\gamma, p} G_{M}^{s}}{\sigma}, \\
& A_{A}=-\left(1-4 \sin _{\theta_{W}}^{2}\right) \sqrt{\tau(1+\tau)\left(1-\epsilon^{2}\right)} \frac{G_{A}^{e} G_{M}^{\gamma, p}}{\sigma},
\end{aligned}
$$

with $\sigma=\epsilon\left(G_{E}^{\gamma, p}\right)^{2}+\tau\left(G_{M}^{\gamma, p}\right)^{2}$ representing the unpolarized proton cross section. The form factor $G_{A}^{e}$ implicitly contains higher order radiative corrections for the proton axial current and is discussed in Section 2.3.

An alternative parameterization is in terms of isoscalar and isovector weak radiative corrections for the vector form factors. The proton and neutron radiative corrections are given to first order in $\rho-1$ and $\kappa-1$ by

$$
\begin{aligned}
& R_{V}^{p}=\rho-1-(\kappa-1) \frac{4 \sin ^{2} \theta_{W}}{1-4 \sin ^{2} \theta_{W}}, \\
& R_{V}^{n}=\rho-1 .
\end{aligned}
$$


The first order neutral vector form factor was given in Eqn. 2.31. The full expression for $G_{E, M}^{Z}$, including the electroweak radiative corrections is [BPS05]

$$
G_{E, M}^{Z p}=\left(1-4 \sin ^{2} \theta_{W}\right)\left(1+R_{V}^{p}\right) G_{E, M}^{\gamma p}-\left(1+R_{V}^{n}\right) G_{E, M}^{\gamma n}-G_{E, M}^{s}
$$

The full asymmetry for a nucleon, $N$, in terms of the vector $R$ parameters can be written as $\left[\mathrm{M}^{+} 94\right][\mathrm{LMRM} 07]$ :

$$
\begin{aligned}
A_{N}= & -\frac{G_{F} Q^{2}}{4 \pi \alpha \sqrt{2}} \frac{1}{\left[\epsilon\left(G_{E}^{N}\right)^{2}+\tau\left(G_{M}^{N}\right)^{2}\right]} \\
\times & \left\{\left(\epsilon\left(G_{E}^{N}\right)^{2}+\tau\left(G_{M}^{N}\right)^{2}\right)\left(1-4 \sin ^{2} \theta_{W}\right)\left(1+R_{V}^{p}\right)\right. \\
& -\left(\epsilon G_{E}^{p} G_{E}^{n}+\tau G_{M}^{p} G_{M}^{n}\right)\left(1+R_{V}^{n}\right) \\
& -\left(\epsilon G_{E}^{N} G_{E}^{s}+\tau G_{M}^{N} G_{M}^{s}\right)\left(1+R_{V}^{(0)}\right) \\
& \left.-\left(1-4 \sin ^{2} \theta_{W}\right) \epsilon^{\prime} G_{M}^{N} G_{A}^{e}\right\} .
\end{aligned}
$$

\subsection{Axial Form Factor and the Anapole Moment}

As previously mentioned, in neutrino-nucleon scattering, $G_{A}^{Z}$ is a very good approximation of the axial form factor. In electron scattering however, electroweak radiative corrections to the axial current are large. For clarity, the notation $G_{A}^{e} \equiv$

$G_{A}^{Z}$ is used to denote the radiatively corrected axial form factor seen in electron scattering.

Including higher order electroweak corrections in the expression for the axial form factor, modifies Eqn. 2.37 in the following manner [BPS05];

$$
G_{A}^{e}=-\tau_{3}\left(1+R_{A}^{T=1}\right) G_{A}+\sqrt{3} R_{A}^{T=0} G_{A}^{8}+G_{A}^{s}\left(1+R_{A}^{(0)}\right) .
$$


There is now a term proportional to an $\mathrm{SU}(3)$ isoscalar octet form factor $G_{A}^{8}$, which at tree level is zero. The isovector term $G_{A}$ may be written as

$$
G_{A}=\left(\frac{g_{A}}{g_{V}}\right) G_{D}
$$

where

$$
G_{D}\left(Q^{2}\right)=\frac{1}{\left(1+Q^{2} / M_{A}^{2}\right)^{2}}
$$

uses a dipole form to parametrize the $Q^{2}$ dependence of $G_{A}$. The ratio of the axial and vector coupling constants, $\frac{g_{A}}{g_{V}}=1.2695(29)$ [AoPDG08], is well known at zero momentum transfer from $\beta$-decay and other charged-curent weak interaction processes, such as $\nu_{\mu}+n \rightarrow p+\mu^{-}$from quasi-elastic neutrino scattering from deuterium. The axial mass, $M_{A}=1.014 \pm 0.014(\mathrm{GeV} / \mathrm{c})^{2}$, was determined by fitting neutrino-deuterium data and comparing that result with calculations from pion electroproduction experiments corrected for hadronic effects [BABB08]. The pion and the neutrino data are in close agreement, so although $M_{A}$ can not at this time be determined from first principles, it can be described accurately phenomenologically for $Q^{2}<1(\mathrm{GeV} / \mathrm{c})^{2}$.

The dipole expression of $G_{A}$ can then be used to determine an axial radius in a low momentum expansion of $G_{A}$ with $Q^{2}$ [BFHM98],

$$
\left\langle r_{A}^{2}\right\rangle=\left.\frac{6}{g_{A}} \frac{d G_{A}}{d Q^{2}}\right|_{Q^{2}=0}=\frac{12}{M_{A}}
$$

The $\mathrm{SU}(3)$ octet form factor $G_{A}^{8}$ at $Q^{2}=0$ can be estimated from the ratio of axial vector to vector couplings in hyperon $\beta$ decay which, assuming $\mathrm{SU}(3)$ flavor 
symmetry, can be related to the hyperon $F$ and $D$ coefficients $\left[\right.$ Bei05] $\left[\mathrm{G}^{+} 00\right]$,

$$
G_{A}^{8}(0)=\frac{(3 F-D)}{2 \sqrt{3}}=0.585 \pm 0.025
$$

The isoscalar strange axial form factor $G_{A}^{s}$ reduces at $Q^{2}=0$ to $G_{A}^{s}=\Delta s$, where $\Delta s$ is the fraction of nucleon spin carried by the strange quarks $(s+\bar{s})$. The

$Q^{2}$ behavior of both $G_{A}^{8}$ and $G_{A}^{s}$ has not been measured. Generally, it is assumed to have the same dipole form as the isovector form factor $G_{A}$, resulting in the following expression for the axial form factor,

$$
G_{A}^{e}=G_{A}^{D}\left[\frac{G_{A}}{G_{V}} \tau_{3}\left(1+R_{A}^{T=1}\right)+\frac{3 F-D}{2} R_{A}^{T=0}+\Delta s\left(1+R_{A}^{(0)}\right)\right] .
$$

\subsubsection{The Anapole Contribution}

The anapole moment is a parity-violating electromagnetic interaction where along with a photon exchange between the electron and the nucleon, a weak parityviolating hadronic interaction also occurs [ZPHRM00]. The electroweak radiative correction associated with the anapole moment was referred to earlier as the "many quark" correction to the axial form factor. Zhu calculated the contributions of the anapole moment to $R_{A}^{T=1}$ and $R_{A}^{T=0}$ using heavy baryon chiral perturbation theory to order $p^{3}$ [ZPHRM00]:

$$
\begin{aligned}
& \left.R_{A}^{T=1}\right|_{\text {anapole }}=-\frac{8 \sqrt{2} \pi \alpha}{G_{\mu} \Lambda_{\chi}^{2}} \frac{1}{1-4 \sin ^{2} \theta_{W}} \frac{a_{s}}{g_{A}}, \\
& \left.R_{A}^{T=0}\right|_{\text {anapole }}=-\frac{8 \sqrt{2} \pi \alpha}{G_{\mu} \Lambda_{\chi}^{2}} \frac{1}{1-4 \sin ^{2} \theta_{W}} \frac{a_{v}}{g_{A}}
\end{aligned}
$$

where $\Lambda_{\chi}=4 \pi F_{\pi}$ is the chiral symmetry breaking scale and the anapole moment is given by the quantity $a_{s}+\tau_{3} a_{v}$. The relative importance of the anapole interaction 
is clearly seen in the $\frac{1}{1-4 \sin ^{2} \theta_{W}} \approx 10$ enhancement to the correction.

Liu, Mckeown, and Ramsey-Musolf [LMRM07] updated the isovector and isoscalar electroweak axial radiative corrections (Table 2.2) calculated by Zhu et al [ZPHRM00], using the 2007 PDG value for the weak mixing angle. Their result shows that the theoretical uncertainty in the total $R_{A}$ is large compared to the onequark corrections, demonstrating the importance of measuring $G_{A}^{e}$ during the $G^{0}$ backward angle experiment and constraining $R_{A}^{T=1}$.

Table 2.2: The "one quark", "many quark", and total corrections to the axial charges in the $\overline{M S}$ scheme.

\begin{tabular}{|c|c|c|}
\hline \hline & $R_{A}^{T=1}$ & $R_{A}^{T=0}$ \\
\hline \hline One quark & -0.172 & -0.253 \\
Many quark & $-0.086(0.35)$ & $0.014(0.20)$ \\
Total & $-0.258(0.35)$ & $-0.239(0.20)$ \\
\hline \hline
\end{tabular}

\subsection{The Deuteron}

In order to determine $G_{A}^{e}, G_{E}^{S}$, and $G_{M}^{S}$ experimentally, the three unknowns requiring a measurement are $G_{E}^{Z}, G_{M}^{Z}$, and $G_{A}^{Z}$ (see Eqn. 2.63). Two asymmetry measurements can be made using a proton target but with two different kinematic settings, i.e. forward angle or backward angle scattering. Another equation with the same unknowns and same $Q^{2}$ is also required. A liquid deuterium target was selected. Not only is it stable, unlike neutrons, but it is isoscalar, so the $G_{M}^{s}$ term is reduced.

In the static approximation, the nucleons in the deuteron are treated as free, 
non-interacting particles. The proton and neutron asymmetries add incoherently, resulting in the following expression for the parity violating asymmetry from quasielastic electron scattering from deuterium, $A_{d}$ [HPD92]:

$$
A_{d}=\frac{\sigma_{p} A_{p}+\sigma_{n} A_{n}}{\sigma_{p}+\sigma_{n}}
$$

where $\sigma_{n(p)}$ is the cross section for elastic electron-neutron (proton) scattering. This simplification does not account for nuclear structure effects and their potential impact on the asymmetry. Hadjimichael, Poulis, and Donnelly studied the impact of the nuclear effects at the SAMPLE experiment's kinematics and found that the difference to the asymmetry was at the 2-3\% level [HPD92]. A more recent paper by Diaconescu, Schiavilla, and von Klock [DSvK01] used the Argonne $\nu_{18}$ potential at $Q^{2} \approx 0.1(\mathrm{GeV} / \mathrm{c})^{2}$. The authors reported that the two-body contributions to the asymmetry are small, $\approx 0.2 \%$ around the quasi-elastic peak, but increase to 3 $\%$ away from the peak.

Because of the $\tau_{3}$ term in front of the $R_{A}^{T=1}$ in Eqn. 2.69, and the relative size of $G_{M}^{p} \approx 2.79$ and $G_{M}^{n} \approx-1.91$, the $R_{A}^{T=1}$ term is enhanced, and the $R_{A}^{T=0}$ term is suppressed in the deuterium asymmetry measurement.

\subsubsection{Two Boson Exchange Correction}

Because the expected size of the extracted strange vector form factors is small and because the proton's weak charge is also small, the relative importance of two boson exchange (TBE) effects in a parity-violating electron scattering measurement

are enhanced [TBM09]. Although a suppressed higher order interaction, two-photon 
exchange (TPE) was found to have a significant impact in resolving the discrepancy between the electric to magnetic proton form factor ratio measurements using the Rosenbluth separation technique [BMT05]. Tjon, Blunden, and Melnitchouk [TBM09] and Negata et al [NZKY09], both recently calculated the electroweak radiative corrections including corrections arising from the interference of first order and TBE diagrams, both electromagnetic $(\gamma, \gamma)$ and $\operatorname{electroweak}(\gamma, Z)$. The work from both groups is in good agreement with one another and the effect on the final asymmetry result for the $G^{0}$ backward angle measurement is less than $1 \%$.

Following the procedure of Tjon et al., the calculation was made by changing the amplitudes in Eqn. 2.45 in the following manner,

$$
\begin{aligned}
\mathcal{M}^{E M} & \rightarrow \mathcal{M}^{E M}+\mathcal{M}^{\gamma \gamma} \\
\mathcal{M}^{N C} & \rightarrow \mathcal{M}^{N C}+\mathcal{M}^{\gamma Z}+\mathcal{M}^{Z \gamma}
\end{aligned}
$$

where the two-photon and $\gamma Z$ exchange amplitudes are given explicitly in [TBM09]. The relative corrections from the $Z(\gamma \gamma), \gamma(\gamma Z)$, and $\gamma(\gamma \gamma)$ interference terms are identified as [TBM09]

$$
\begin{aligned}
\delta_{Z(\gamma \gamma)} & =\frac{2 \mathcal{R}\left(\mathcal{M}^{Z^{*}} \mathcal{M}^{\gamma \gamma}\right)}{2 \mathcal{R}\left(\mathcal{M}^{Z^{*}} \mathcal{M}^{\gamma}\right)} \\
\delta_{\gamma(\gamma Z)} & =\frac{2 \mathcal{R}\left(\mathcal{M}^{\gamma^{*}} \mathcal{M}^{\gamma Z}+\mathcal{M}^{\gamma^{*}} \mathcal{M}^{Z \gamma}\right)}{2 \mathcal{R}\left(\mathcal{M}^{\gamma^{*}} \mathcal{M}^{Z}\right)} \\
\delta_{\gamma(\gamma \gamma)} & =\frac{2 \mathcal{R}\left(\mathcal{M}^{\gamma *} \mathcal{M}^{\gamma \gamma}\right)}{\mid\left(\left.\mathcal{M}^{\gamma}\right|^{2}\right.}
\end{aligned}
$$

In order to apply their TBE correction, it is necessary to first remove the $Q^{2}=0$ hadronic, or low-mass portion of the TBE in the terms $\rho$ and $\kappa$ which are then used to calculate the $R$ factor corrections [TBM09]. The low-mass portion of 
the corrections are referred to as $\Delta \rho_{M S}$ and $\Delta \kappa_{M S}$; these values are shown in Table 2.3 and details of the calculation are provided in Sec. 2.5.

The correction to Eqn. 2.59 is then

$$
A=(1+\delta) A^{0} \equiv\left(\frac{1+\delta_{Z(\gamma \gamma)}+\delta_{\gamma(Z \gamma)}}{1+\delta_{\gamma(\gamma \gamma)}}\right) A^{0}
$$

where $A^{0}$ is given in Eqn. 2.63.

\subsection{Electroweak Parameters}

This section presents a summary of the parameters and calculations used to determine the electroweak correction parameters, the $R$ factors, which were applied to the $G^{0}$ backward angle measurement to extract the axial and vector strange form factors. All values are calculated in the $\overline{M S}$ scheme.

To arrive at the $R$ factors, the values in Table 2.3 are used to first compute a set of constants, $C_{1 u}, C_{1 d}, C_{2 u}$, and $C_{2 d}$ (Eqn. 2.79) found in the Particle Data Book (PDG) that describe the coupling of the electron current to the quark current [AoPDG08]. These relations are:

$$
\begin{aligned}
& C_{1 u}=\rho_{1}^{*}\left(-\frac{1}{2}+\frac{4}{3} \kappa_{1}^{*} \hat{s}_{Z}^{2}\right)+\lambda_{1 u}, \\
& C_{1 d}=\rho_{1}^{*}\left(\frac{1}{2}-\frac{2}{3} \kappa_{1}^{*} \hat{s}_{Z}^{2}\right)+\lambda_{1 d}, \\
& C_{2 u}=\rho_{2}^{*}\left(-\frac{1}{2}+2 \kappa_{2}^{*} \hat{s}_{Z}^{2}\right)+\lambda_{2 u}, \\
& C_{2 d}=\rho_{2^{*}}\left(\frac{1}{2}-2 \kappa_{2}^{*} \hat{s}_{Z}^{2}\right)+\lambda_{2 d},
\end{aligned}
$$

where $\rho_{i}^{*}=\rho_{i}-\Delta \rho_{M S}$ and $\kappa_{i}^{*}=\kappa_{i}-\Delta \kappa_{M S}$. The axial and vector quark charges 
Table 2.3: Parameters used to calculate the electroweak radiative corrections.

\begin{tabular}{|c|c|c|}
\hline \hline Quantity & Value & Reference \\
\hline \hline$G_{F}$ & $1.16639 \times 10^{-5}$ & {$[$ AoPDG08] } \\
$M_{A}$ & $1.014 \pm 0.014(\mathrm{GeV} / \mathrm{c})^{2}$ & {$[$ BABB08] } \\
$\hat{s}_{Z}^{2}$ & $0.23120(15)$ & {$[$ AoPDG08] } \\
$g_{A}$ & $1.2695 \pm 0.0029$ & {$[$ AoPDG08] } \\
$3 F-D$ & $0.585 \pm 0.025$ & {$[$ AoPDG08] } \\
$g_{A}$ & $1.2695 \pm 0.0029$ & {$\left[\mathrm{G}^{+} 00\right]$} \\
$\rho_{1}$ & 0.9875 & {$[$ AoPDG08] } \\
$\rho_{2}$ & 1.0004 & {$[$ AoPDG08] } \\
$\kappa_{1}$ & 1.0025 & {$[$ AoPDG08] } \\
$\kappa_{2}$ & 1.0298 & {$[$ AoPDG08] } \\
$\lambda_{1 u}$ & $-1.80 \times 10^{-5}$ & {$[$ AoPDG08] } \\
$\lambda_{1 d}$ & $3.6 \times 10^{-5}$ & {$[$ AoPDG08] } \\
$\lambda_{2 u}$ & -0.0121 & {$[$ AoPDG08] } \\
$\lambda_{2 d}$ & 0.0026 & {$[$ AoPDG08] } \\
$\Delta \rho_{M S}$ & -0.00071 & {$[$ TBM09] } \\
$\Delta \kappa_{M S}$ & -0.001027 & {$[$ TBM09] } \\
\hline \hline
\end{tabular}

can be expressed in terms of the $C$ parameters,

$$
\begin{aligned}
c_{V}^{u, c, t} & =-2 C_{1 u}, & c_{V}^{d, s, b} & =-2 C_{1 d}, \\
c_{A}^{u, c, t} & =\frac{2 C_{2 u}}{1-4 \sin ^{2} \theta_{w}}, & c_{A}^{d, s, b} & =\frac{2 C_{2 d}}{1-4 \sin ^{2} \theta_{w}} .
\end{aligned}
$$

The quark charges are related to the $R$ factors through the six weak nucleon charges, 
which provide the link between the quark charges and the $R$ factors,

$$
\begin{aligned}
Q_{W}^{p} & =2 c_{V}^{u}+c_{V}^{d}=\left(1+R_{V}^{p}\right)\left(1-4 \sin ^{2} \theta_{w}\right), \\
Q_{W}^{n} & =2 c_{V}^{d}+c_{V}^{u}=-\left(1+R_{V}^{n}\right), \\
Q_{W}^{(0)} & =c_{V}^{u}+c_{V}^{d}+c_{V}^{s}=-\left(1+R_{V}^{0}\right), \\
Q_{A}^{T=1} & =\frac{1}{2}\left(c_{A}^{u}-c_{A}^{d}\right)=-\left(1+R_{A}^{T=1}\right), \\
Q_{A}^{T=0} & =\sqrt{3}\left(c_{A}^{u}+c_{A}^{d}\right)=\sqrt{3}\left(1+R_{A}^{T=0}\right), \\
Q_{A}^{(0)} & =c_{A}^{u}+c_{A}^{d}+c_{A}^{s}=\left(1+R_{A}^{0}\right) .
\end{aligned}
$$

Table 2.4 shows the values for the $R$ factors ("one quark" only) for hydrogen.

Table 2.4: Radiative correction factors ("one quark").

\begin{tabular}{|c|c|}
\hline \hline$R$ Factor & Value \\
\hline \hline$R_{V}^{p}$ & -0.0546 \\
$R_{V}^{n}$ & -0.0117 \\
$R_{V}^{(0)}$ & -0.0112 \\
$R_{A}^{T=1}$ & -0.171 \\
$R_{A}^{T=0}$ & -0.253 \\
$R_{A}^{(0)}$ & -0.550 \\
\hline \hline
\end{tabular}

\subsection{Theoretical Predictions of $G_{E}^{S}$ and $G_{M}^{S}$}

The $G^{0}$ measurement of the vector strange form factors is independent of any theoretical models predicting the presence or behavior of strange quarks in the nucleons. One can argue that this makes the theoretical predictions even more interesting. The difficulty in calculating static properties of the nucleon is that 
the strong coupling constant $\left(\alpha_{s}\right)$ is large at low energies and therefore it is not possible to use a perturbative expansion in $\alpha_{s}$ to describe the interaction. Because of the difficulty of a straight-forward calculation of the strange quark contribution to nucleon properties, there have been a wide variety of approaches to making an effective calculation. A survey of the most popular methods is presented below.

Most models focus on predicting the contribution to the strange magnetic moment, $\mu_{s}$, and the strangeness radius, $r_{s}^{2}$, both defined at $Q^{2}=0$. The strangeness radius gives the mean square radius of the strange "charge" distribution. A positive value implies that the $s$ quark is further away from the center of the nucleon than $\bar{s}$ and visa versa [BH01]. The expressions for these quantities are:

$$
\begin{aligned}
\mu_{s} & =G_{M}^{s}\left(Q^{2}=0\right) \\
\left\langle r_{s}\right\rangle^{2} & \equiv-6 \frac{d G_{E}^{s}}{d Q^{2}}\left(Q^{2}=0\right)
\end{aligned}
$$

\subsubsection{Chiral Perturbation Theory}

Chiral perturbation theory (CHPT) is a powerful tool that capitalizes on the QCD Lagrangian having an approximate $S U(3)_{L} \times S U(3)_{R}$ symmetry in the limit where the light quark masses vanish. Because the physical masses of the three lightest quarks are much less than the hadronic scale $(\approx 1 \mathrm{GeV})$, the massless approximation is reasonable. Chiral symmetry is used to relate one set of observables to another, or to draw on one set of measured quantities to predict another [RMI97].

This strategy breaks down in the flavor-singlet channel because the coefficients of the relevant flavor-singlet operators in the chiral Lagrangian, which contain in- 
formation on short-distance hadronic effects, cannot be determined from existing measurements using chiral symmetry [RMI97]. Leading order, long-distance contributions are calculable however for $\mu_{s}$ and $r_{s}$, but it is not clear that these contributions dominate the short-distance effects. Therefore in order to determine the strangeness contribution of the nucleon, model-dependent assumptions are necessary [RMI97].

There are a number of hadronic models, but two that will be discussed here are variations of "pole" and "loop" models. The primary shared feature of these models is the use of a strange intermediate hadronic state to approximate the nucleon's strangeness content.

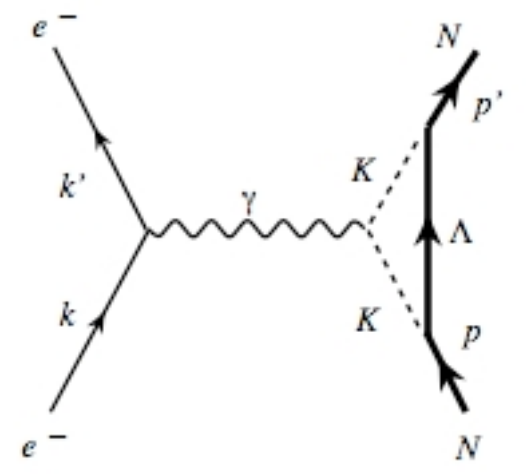

Figure 2.3: The primary Feynman diagram for a loop-model calculaton. A nucleon

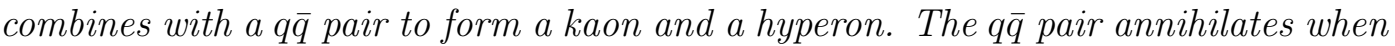
the kaon and hyperon recombine leaving the original nucleon.

\subsubsection{Loop Models}

In the Kaon Loop model, also referred to as the kaon cloud model, a nucleon combines with a $q \bar{q}$ pair to form a meson and an intermediate baryon state (see 
Fig. 2.3). The $q \bar{q}$ pair annihilates when the meson and baryon recombine leaving the original nucleon. One of the appeals of this model is the physical interpretation of the nucleon charge radius, where the model characterizes a spatial asymmetry with a non-zero charge distribution for $s$ and $\bar{s}$. The intermediate strange mesonbaryon state (typically modeled as a kaon and a hyperon) allows the $s$ and $\bar{s}$ to spatially separate because of the mass difference between the two intermediate state particles [RMI97]. Another motivation for this model came from the success of a pion loop calculation of the nucleon's electromagnetic form factors carried out by Bethe and DeHoffman [BH55]. When their results were reported, there was surprising agreement with experimental values for both the nucleon's charge radii and magnetic moments, despite the large $\pi N$ coupling which enters the perturbative calculation. This led to the belief that the pion cloud dominates the nucleon's isovector electromagnetic moments and that it is sufficiently described using a oneloop calculation. It was thought that if this were also the case for the strangeness sector, then the kaon cloud would provide the dominant contribution to the strange charge and magnetic moment [RMI97].

One approach for using kaon loop calculations was made by Koepf, Henley, and Pollock [KHP92]. They used bag models, both the "cloudy" constituent quark model and the cloudy bag model $(\mathrm{CBM})$ to describe the hadrons. The size and structure of the nucleon bag was contained in a form factor, $v(k)$, with the size of the bag serving as the only unknown parameter. The model bag size was extracted using fits to the nucleon magnetic moments and charge radii. After fixing the size parameter, kaon loop calculations were completed. 
Kaon loops introduce divergences that are typically handled with a momentum cut-off in the loop integral [DGH92]. Ramsey-Musolf and Burkardt performed a loop calculation within the context of the $\mathrm{SU}(3)$ linear $\sigma$ model where the leading strangeness moments are ultraviolet finite. The calculation was performed by including hadronic form factors at the meson-nucleon vertices, using results of fits to baryon-baryon scattering in the one meson exchange approximation [MB94].

Geiger and Isgur [GI97] provided a follow-on kaon loop calculation using a non-relativistic quark model with yet another variation. Their calculation summed over a complete set of strange intermediate states, rather than just a few low-lying states, which provided a consistency with the OZI rule. The authors point out that their results are not predictive of $\mu_{s}$ because their calculation ignored pure OZI-forbiddden effects.

\subsubsection{Pole Models}

Based on analyticity and causality, dispersion relations (DR) relate the real parts of the form factors to integrals involving their imaginary parts. The imaginary parts, or spectral functions, contain information on the contributions to the form factor dynamics made by various states in the hadronic spectrum [HRM99].

For example, to obtain the dispersion relation for the Dirac form factor, $F_{i}(t)$, where $t$ is real, the assumption is made than an analytic continuation $F_{i}(z)$ exists

in the upper-half plane that approaches $F_{i}(t)$ as $z \rightarrow t+i e$ and that $\frac{F_{i}(z)}{z^{n}} \rightarrow 0$ as $z \rightarrow \infty$ for non-negative integers in the upper-half plane. Using Cauchy's theorem, 
a subtracted DR is shown for $F_{1}$ and an unsubtracted one for the Pauli form factor, $F_{2}$ :

$$
\begin{aligned}
& F_{1}(t)=F_{1}(0)+\frac{t}{\pi} \int_{9 m_{\pi}^{2}}^{\infty} \frac{\operatorname{Im} F_{1}\left(t^{\prime}\right)}{t^{\prime}\left(t^{\prime}-t\right)} d t^{\prime}, \\
& F_{2}(t)=\int_{9 m_{\pi}^{2}}^{\infty} \frac{I m F_{2}\left(t^{\prime}\right)}{t^{\prime}\left(t^{\prime}-t\right)} d t^{\prime} .
\end{aligned}
$$

The lower limit of integration is given by the threshold of the lightest intermediate state contributing to the form factors, the $3 \pi$ state [HRM99].

Pole models take a dispersion analysis approach, and are based on the work of Höller et al., $\left[\mathrm{H}^{+} 76\right]$, where the basic premise is that the exchanged boson fluctuates into an isoscalar meson, either an $\omega$ or a $\phi$, and then the meson interacts with the nucleon (see Fig. 4.9.2). Both the $\omega$ and the $\phi$ are linear combinations of strange

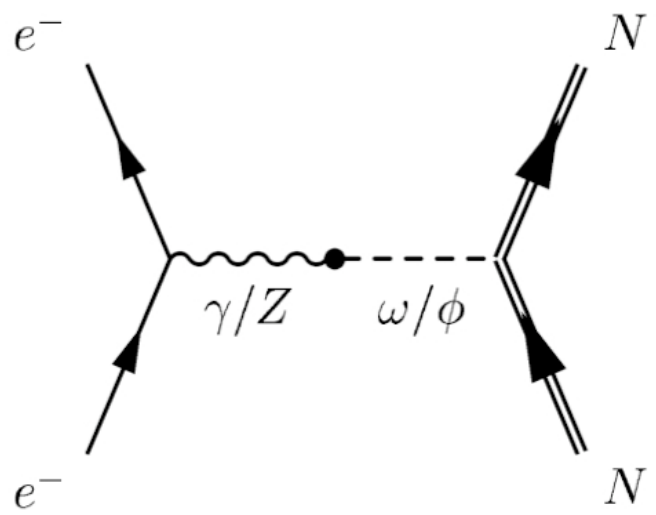

Figure 2.4: The primary Feynman diagram for a pole-model calculaton.

and non-strange base states:

$$
\begin{aligned}
& |\omega\rangle=\cos (\eta)\left|\omega_{o}\right\rangle-\sin (\eta)\left|\phi_{o}\right\rangle, \\
& |\omega\rangle=\sin (\eta)\left|\omega_{o}\right\rangle+\cos (\eta)\left|\phi_{o}\right\rangle,
\end{aligned}
$$

where $\phi_{o}=|s \bar{s}\rangle, \omega_{o}=\frac{1}{\sqrt{2}}(|u \bar{u}\rangle+|d \bar{d}\rangle)$, and $\eta=0.053 \pm 0.005$ is the mixing angle [Jaf89].

Vector meson dominance (VMD) is a special application of a dispersion relation with the assumption that the nucleon matrix element can be written as a summation 
over intermediate vector states. For example,

$$
\begin{aligned}
& F_{1}^{a}\left(q^{2}\right)=F_{1}^{a}+\sum_{V} \frac{q^{2} a_{V}^{a}}{m_{V}^{2}-q^{2}}, \\
& F_{2}^{a}\left(q^{2}\right)=\sum_{V} \frac{m_{V}^{2} b_{V}^{a}}{m_{V}^{2}-q^{2}},
\end{aligned}
$$

where $m_{V}$ is the mass of an intermediate meson $V$. Jaffe calculated the strangeness radius and magnetic moment using a 3-pole fit to experimental data for the spectral function of the isoscalar nucleon form factors. His first and second terms represented the coupling of nucleons to $\omega(780)$ and $\phi(1020)$ mesons. The third term represented contributions from other mass states.

\subsubsection{Loops and Poles}

Loop and pole predictions for the strangeness radius are opposite sign, and the magnitude of the loop prediction for the strangeness radius is about 20 times smaller for the Dirac radius than that of the pole prediction. This motivated Cohen, Forkel, and Nielsen to attempt to establish a link between the pole and loop pictures, by combining the VMD model in the $\omega$ and $\phi$ sector $\left(Y T=0, J^{P C}=1^{--}\right)$with Musolf and Burkardt's loop calculation. They calculated the nucleon strange matrix

elements using kaon loops, and used Höller's empirical fits for the isoscalar matrix elements, which were then combined using the VMD assumption with only $\omega$ and $\phi$ poles [CFN93]. 


\subsubsection{Lattice QCD}

Lattice QCD is a non-perturbative computational method based on a Feynman path integral approach to quantum field theory. Computations are performed on a lattice of space-time through intensive use of numerical integrations. Quarks and gluons reside on lattice points and can only travel along lines between them. This approximation approaches continuum QCD as the spacing between the lattice points approaches zero.
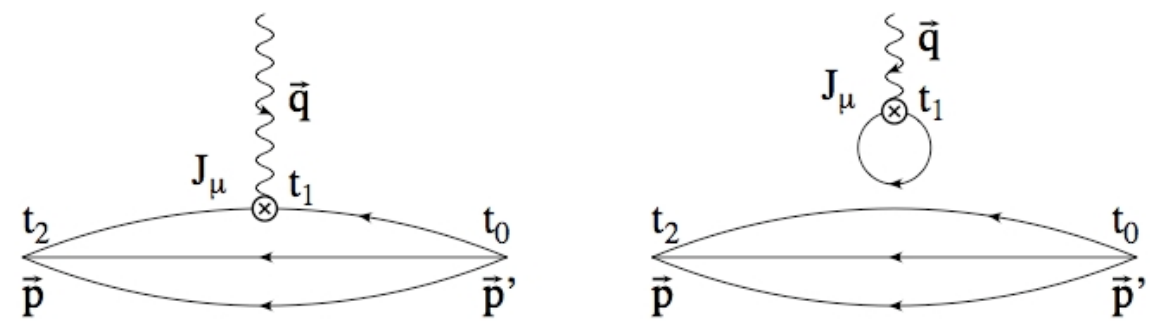

Figure 2.5: Three point function representations. The connected insertion (left) compared to the disconnected insertion (right) $\left[D^{+} 09 a\right]$.

Unlike the models previously discussed, lattice QCD offers a first-principle calculation. One of the major difficulties with using lattice calculations to calculate the strange electromagnetic form factors, is that the calculation requires the evaluation of the disconnected insertion (DI). The DI calculation (Fig. 2.5 (right)) is a much more difficult calculation compared to the connected insertion (Fig. 2.5 (left)) calculation, because the straightforward DI calculation requires all-to-all propagators, and is prohibitively expensive, $\left[\mathrm{D}^{+} 09 \mathrm{a}\right]$. Recently, Doi et al., published the first full QCD lattice simulation of the direct insertion calculation with high statistics. Their result, along with calculations from other analyses is shown in Table 2.5. 
Table 2.5: Summary of theoretical predictions for $\mu_{s} s$.

\begin{tabular}{|c|c|c|}
\hline \hline Type of Calculation & $\mu_{s}$ & Reference \\
\hline \hline Lattice QCD & $-0.017(25)(07)$ & {$\left[\mathrm{D}^{+} 09 \mathrm{a}\right]$} \\
Dispersion relation with Pole ansatz & $-0.31(9)$ & {$[\mathrm{Jaf} 89]$} \\
Dispersion relation with Kaon Clouds & $-(0.15 \rightarrow 0.51)$ & {$[\mathrm{HRM99}]$} \\
Quark Model & 0.035 & {$[\mathrm{GI} 97]$} \\
Chiral Quark-Soliton Model & $0.08-0.13$ & {$[$ SKUG06] } \\
\hline \hline
\end{tabular}

The next chapter is a discussion of experimental measurements taken prior to the $G^{0}$ backward angle experiment that were designed to probe the strange quark sea. A summary of results is included. 


\section{Chapter 3}

\section{Summary of Existing Measurements}

The first measurement of parity-violating asymmetries from scattering polarized electrons from deuterium and hydrogen liquid targets was conducted at the Stanford Linear Accelerator (SLAC) in the late 1970's $\left[\mathrm{P}^{+} 78\right]$. The asymmetry measurement was made at $Q^{2}=1.6(\mathrm{GeV} / \mathrm{c})^{2}$ and the value they obtained for $\sin ^{2} \theta_{w}$ was in good agreement with that predicted by electroweak theory. Their observation and first measurement of non-conservation of parity in electron-nucleon scattering added important new information to our understanding of the nature of neutral currents and gauge theories.

Since then, there have been numerous asymmetry measurements involving scattered polarized electrons from various polarized and unpolarized targets, at both forward and backward angles. However, there are three experiments in addition to $G^{0}$, with the intent of probing the strange quark sea and obtaining measurements of the electromagnetic strange form factors. These three experiments are:

- SAMPLE performed at the MIT-Bates Linear Accelerator Center (SAMPLE I 1998, Sample II 1999, SAMPLE III 2001-2002)

- HAPPEx performed in Hall A at Thomas Jefferson National Accelerator Facility (HAPPEx 1 1998,1999; HAPPEx II [HAPPEx-H 2004 and 2005, HAPPExHe 2004 and 2005], HAPPEx III 2009) 
- PVA4 at the Mainzer Mikrotron accelerator facility (MAMI) in Mainz (2004 to present)

Each of these experiments is sensitive to some combination of $G_{E}^{s}, G_{M}^{s}$, and $G_{A}^{e(T=1)}$. An experimental overview along with the published results to date from these experiments is presented in this chapter. Also presented are the results from the $G^{0}$ forward angle measurement.

\subsection{SAMPLE}

SAMPLE (Singlet Anomalous Moment of the Proton using Longitudinally Polarized Electrons) was the first experiment designed to measure the strange quark's contribution to the vector form factors $\left[\mathrm{M}^{+} 97\right],\left[\mathrm{S}^{+} 00\right],\left[\mathrm{S}^{+} 04\right],\left[\mathrm{I}^{+} 04\right]$, and [BPS05]. Three measurements were taken, all in the backward angle configuration using an unpolarized $40 \mathrm{~cm}$-long cryogenic target. The first measurement was taken in the summer of 1998 with a beam energy of $200 \mathrm{MeV}$ and a liquid hydrogen target. The second measurement was in the summer of 1999, also at $200 \mathrm{MeV}$, but using a liquid deuterium target, and the final measurement was in the winter of 2001-2002 on a liquid deuterium target with a beam energy of $125 \mathrm{MeV}$.

The scattered electrons were detected in an air Čerenkov detector. The detector consisted of 10 large mirrors, each with ellipsoidal curvature to focus the Čerenkov light onto one of 10 shielded photomultiplier tubes, as shown in Fig. 3.1. A remotely controlled light shutter could cover each of the photomultiplier tubes and was used to measure the background. The beam was pulsed at $600 \mathrm{~Hz}$ and the 

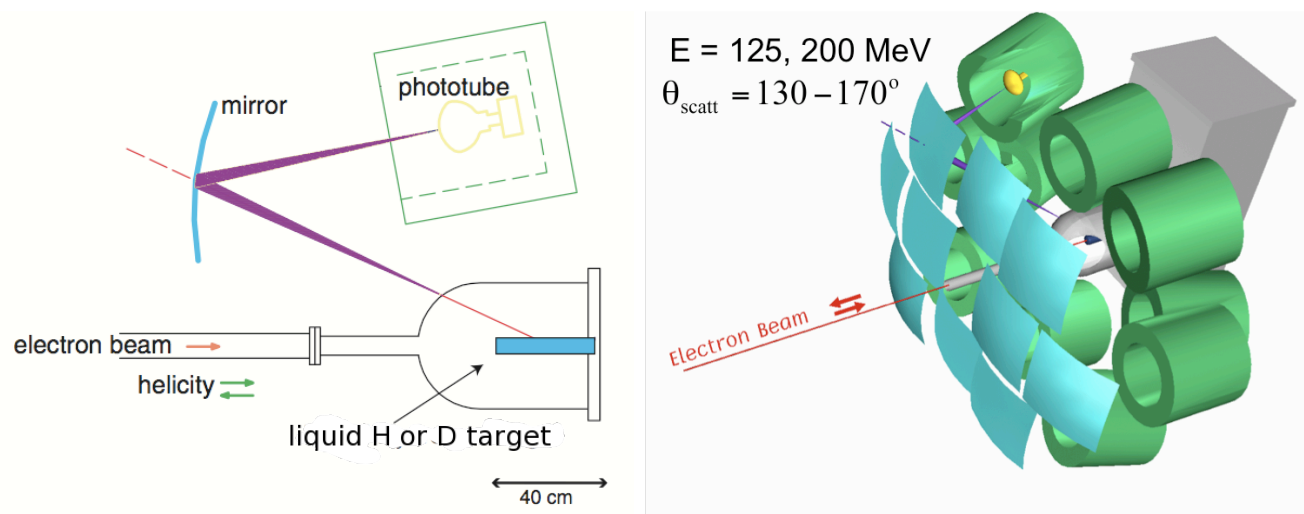

Figure 3.1: Depiction of the SAMPLE experimental apparatus. The Čerenkov photons from backward-scattered electrons were reflected and focused by the mirrors onto the photomultiplier tubes.

average beam current was $40 \mu \mathrm{A}$. The signals from the detector, and various beam monitors, were integrated and digitized for every $25 \mu$ sec beam pulse. The parityviolating asymmetry was determined from the asymmetries in ratios of integrated detector signal to beam intensity for left- and right-handed beam pulses.

The SAMPLE experiment took data in the backward angle mode, $\theta=130^{\circ}-$ $170^{\circ}$, which is most sensitive to $G_{M}^{s}$ and $G_{A}^{e(T=1)}$. The first two SAMPLE measurements were both at $Q^{2}=0.091(\mathrm{GeV} / \mathrm{c})^{2}$ where the measured proton $\left[\mathrm{S}^{+} 04\right]$ and deuteron [BPS05] asymmetries were:

$$
\begin{aligned}
& A_{p}=-5.61 \pm 0.67 \pm 0.88=-5.56+3.37 G_{M}^{s}+1.54 G_{A}^{e(T=1)} \mathrm{ppm} \\
& A_{d}=-7.77 \pm 0.73 \pm 0.72=-7.06+0.77 G_{M}^{s}+1.66 G_{A}^{e(T=1)} \mathrm{ppm}
\end{aligned}
$$

and where "ppm" is parts per million.

These results are shown in Fig. 3.2 as $1 \sigma$ bands in the space of $G_{M}^{s}$ and $G_{A}^{e(T=1)}$ along with an uncertainty band from the theory calculation of $G_{A}^{e(T=1)}=-0.83 \pm 0.26$ 
from [ZPHRM00]. The value of $G_{M}^{s}$ using this value for $G_{A}^{e(T=1)}$ is

$$
G_{M}^{s}\left(Q^{2}=0.1\right)=0.37 \pm 0.20(\text { stat }) \pm 0.26(\text { sys }) \pm 0.07(\text { model })
$$

where the model uncertainty includes uncertainties due to the nucleon electromagnetic and axial form factors.

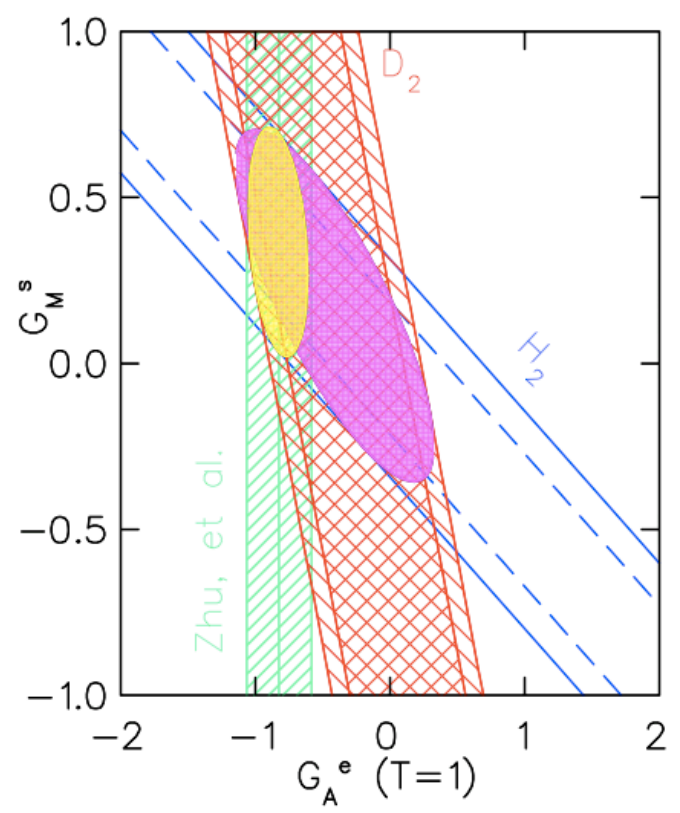

Figure 3.2: Uncertainty bands of $G_{M}^{s}$ and $G_{A}^{e(T=1)}$ at $Q^{2}=0.1(G e V / c)^{2}$ resulting from the two $200 \mathrm{MeV} S A M P L E$ data sets $\left[\mathrm{S}^{+} 04, \mathrm{BPS05}\right]$ labelled " $\mathrm{H}_{2}$ and $\mathrm{D}_{2}$ ". Also shown is the uncertainty band of the theoretical expectation of $G_{A}^{e(T=1)}$ as computed by [ZPHRM00], labelled as "Zhu, et al.", extrapolated to the same momentum transfer. The smaller ellipse corresponds to the $1 \sigma$ overlap of the hydrogen data and the theoretical prediction; the larger one the $1 \sigma$ overlap of the two data sets [ $\left.S^{+} 04\right]$. The inner dashed line $\left(\mathrm{H}_{2}\right)$, and inner straight lines ( $\mathrm{D}_{2}$ and $\mathrm{Zhu}$, et al) represent the 2 $\sigma$ confidence bands.

The third SAMPLE measurement was taken on deuterium with the same experimental apparatus and method as the previous measurements, except that this time the beam energy was $125 \mathrm{MeV}$ with $Q^{2}=0.038$. The measured asymmetry 
was $\left[\mathrm{I}^{+} 04\right]$

$$
A_{d}=-3.51 \pm 0.57 \pm 0.58=-2.14+0.27 G_{M}^{s}+0.76 G_{A}^{e(T=1)} \mathrm{ppm}
$$

The two SAMPLE deuterium asymmetry results are shown in Fig. 3.3 plotted as

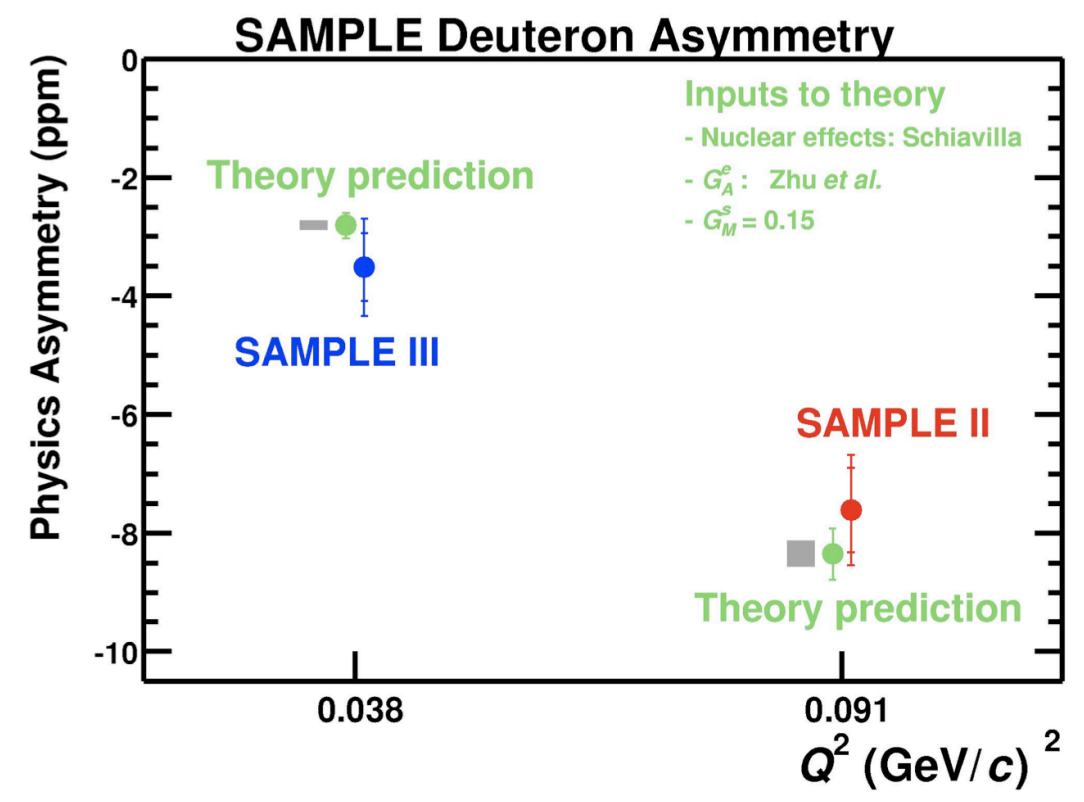

Figure 3.3: The physics asymmetries measured in SAMPLE II and SAMPLE III are plotted as a function of $Q^{2}$ (solid circles). Also plotted (with offset $Q^{2}$ for visibility) is the theoretical prediction using $G_{A}^{e}$ from [ZPHRM00] and $G_{M}^{s}=0.15$ (open circles). The height of the gray rectangles represents the change in the physics asymmetry corresponding to a 0.6 change in $G_{M}^{s}\left[I^{+} 04\right]$.

a function of $Q^{2}$. Also plotted are the theoretical predictions with the value of $G_{A}^{e}$ taken from [ZPHRM00] $\left[G_{A}^{e}\left(Q^{2}=0.038\right)=-0.91 \pm 0.28\right.$ and $G_{A}^{e}\left(Q^{2}=0.091\right)=$ $-0.84 \pm 0.26]$, and $G_{M}^{s}=0.15$. The results from SAMPLE II and SAMPLE III $\left[\mathrm{I}^{+} 04\right]$ both agree with the theoretical prediction of $G_{A}^{e}$. 


\subsection{HAPPEx}

The goal of the Hall A Proton Parity Experiment (HAPPEx) was also to measure the strange quark vector form factors. This was a forward angle measurement sensitive to linear combinations of $G_{e}^{s}$ and $G_{M}^{s}$. The first HAPPEx measurements [A $\left.{ }^{+} 99 \mathrm{~b}\right],\left[\mathrm{A}^{+} 01\right],\left[\mathrm{A}^{+} 04\right]$, covered two run periods, one in 1998 and one in 1999. For the first run period, a $100 \mu \mathrm{A}$ beam of longitudinally polarized $3.36 \mathrm{GeV}$ electrons was scattered from a $15 \mathrm{~cm}$ long liquid hydrogen target. Scattered elastic electrons $\left(\theta \approx 12^{\circ}\right)$ were focused by two identical high resolution spectrometers onto a total-absorption detector comprised of alternating layers of lead and lucite. The spectrometers were able to spatially separate the elastic electrons from the inelastic electrons ensuring a low background. The beam helicity was set every $33.3 \mathrm{~ms}$ and was structured as pairs of consecutive $33 \mathrm{~ms}$ periods with opposite helicity, referred to in the literature as windows. The Čerenkov light from the scattered electrons was collected by a photomultiplier tube, integrated over the duration of the helicity window, and digitized by analog to digital converters (ADC). A schematic of the experimental apparatus is shown in Fig. 3.4.

The asymmetry measured during the 1998 run $\left[\mathrm{A}^{+} 99 \mathrm{~b}\right]$ at $Q^{2}=0.48(\mathrm{GeV} / \mathrm{c})^{2}$ was $A_{p}=-14.5 \pm 2.0$ (stat) \pm 1.1 (syst) ppm, from which the following linear combination of form factors was extracted,

$$
G_{e}^{s}+0.39 G_{M}^{s}=0.023 \pm 0.034(\text { stat }) \pm 0.022(\text { syst }) \pm 0.026\left(\delta G_{E}^{n}\right) .
$$

The last uncertainty was due to the estimated uncertainty in the neutron electric form factor. 


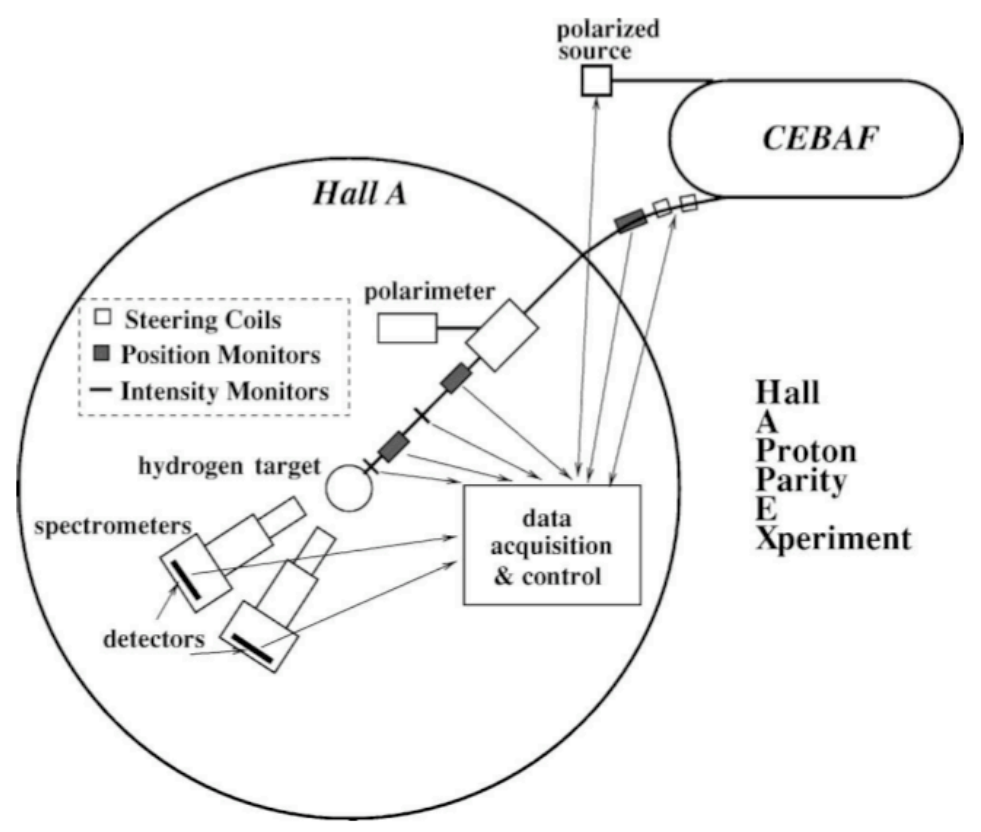

Figure 3.4: A depiction of the HAPPEx apparatus, used for all of the measurements from $\left[A^{+} 04\right]$. Forward scattered electrons are detected by the high-resolution spectrometers.

The HAPPEx I measurement taken in 1999 used a $35 \mu \mathrm{A}$ beam with an energy of $3.3 \mathrm{GeV}$ with $Q^{2}=0.477 \mathrm{GeV}$ using the same experimental apparatus (and $\theta \approx 12^{\circ}$ ) as the 1998 measurement. A new feature however, was much improved beam polarization ( $\mathrm{P}_{e} \approx 70 \%$ versus $\left.\mathrm{P}_{e}=39 \%\right)$ because of the switch to a strained GaAs crystal from a bulk GaAs photocathode in the polarized source. The measured asymmetry was $A=-15.05 \pm 0.98$ (stat) \pm 0.56 (syst) ppm, and both errors were a factor of two smaller than the 1998 HAPPEx results. The value extracted from the data for the linear combination of the strange vector form factors was first reported in $\left[\mathrm{A}^{+} 01\right]$ and was updated in $\left[\mathrm{A}^{+} 04\right]$ as

$$
\left(G_{E}^{s}+0.392 G_{M}^{s}\right)=0.014 \pm 0.020 \pm 0.010
$$

where the first error is experimental and the second arises from the uncertainties in 
electromagnetic form factors.

The experimental configuration of HAPPEx II was similar to HAPPEx I except for a few changes that made it possible to detect scattered electrons at very forward $\left(\theta \approx 6^{\circ}\right)$ lab angles. One change was the addition of superconducting septum magnets that increased the acceptance; another addition was radiation-hard focal plane detectors comprised of alternating layers of brass and quartz which were capable of surviving the increased electron rate seen at forward angles $\left[\mathrm{A}^{+} 06 \mathrm{c}\right]$.

The first HAPPEx II measurements on hydrogen and ${ }^{4} \mathrm{He}$ targets were in 2004 using a $35 \mu \mathrm{A}$ beam of longitudinally polarized $3.03 \mathrm{GeV}$ electrons incident on a $20 \mathrm{~cm}$ target with a scattering angle of $\theta=6.06^{\circ}$. The results from these measurements were published in $\left[\mathrm{A}^{+} 06 \mathrm{c}\right]$ and $\left[\mathrm{A}^{+} 06 \mathrm{~b}\right]$. The final results using data from the hydrogen and ${ }^{4} \mathrm{He}$ targets for both run periods (2004 and 2005) were published in $\left[\mathrm{A}^{+} 07\right]$. The reported asymmetry measurements for the two targets were

$$
\begin{aligned}
& A_{H e}=+6.40 \pm 0.23(\text { stat }) \pm 0.12(\text { syst }) p p m \\
& A_{H}=-1.58 \pm 0.12(\text { stat }) \pm 0.04(\text { syst }) p p m
\end{aligned}
$$

The hydrogen measurements permitted the extraction of the linear combination of the strange vector form factors at $Q^{2}=0.109(\mathrm{GeV} / \mathrm{c})^{2}$ as

$$
\left(G_{E}^{s}+0.09 G_{M}^{s}\right)=0.007 \pm 0.011(\text { stat }) \pm 0.006(\text { syst })
$$

Normally, as previously mentioned, forward angle measurements are sensitive to a linear combination of $G_{E}^{s}$ and $G_{M}^{s}$, but the ${ }^{4}$ He nucleus is spin zero, parityeven, and isoscalar with isospin symmetry, therefore the parity-violating asymmetry 
for elastic electron scattering is only sensitive to $G_{E}^{s}\left[\mathrm{M}^{+} 94\right]$. The parity-violating asymmetry at tree-level is given by $\left[\mathrm{M}^{+} 94\right]$

$$
A_{P V}^{H e}=\frac{G_{F} Q^{2}}{4 \pi \alpha \sqrt{2}}\left(4 \sin ^{2} \theta_{w}+\frac{G_{E}^{s}}{G_{E}^{\gamma^{T=0}}}\right),
$$

where $G_{E}^{\gamma^{T=0}}=\left(G_{E}^{\gamma p}+G_{E}^{\gamma n}\right) / 2$ is the isospin-zero electric form factor, which is known. Using this relationship, the HAPPEx collaboration compared their results with theoretical expectations, extracting

$$
G_{E}^{s}=-0.038 \pm 0.042 \text { stat } \pm 0.010 \text { syst }
$$

at $Q^{2}=0.077(\mathrm{GeV} / \mathrm{c})^{2}$, where the uncertainties in the nucleon electromagnetic form factors govern the last error $\left[\mathrm{A}^{+} 07\right]$.

HAPPEx III took measurements on a hydrogen target at $Q^{2} \approx 0.6(\mathrm{GeV} / \mathrm{c})^{2}$ in the Fall of 2009 and are analyzing data at this time. More information about this experiment can be found in Sec. 6.5.

\section{$3.3 \quad$ PVA4}

The PVA4 experimental program at Mainz $\left[\mathrm{M}^{+} 04\right],\left[\mathrm{M}^{+} 05\right]$, and $\left[\mathrm{B}^{+} 09 \mathrm{a}\right]$ is different from SAMPLE and HAPPEx in that the individually scattered particles are detected and counted using very fast electronics, rather than using an integrated detector signal. Additionally, the PVA4 collaboration designed the experiment so that the spectrometer could be moved, so that measurements at both forward and backward angles could be performed. Both of these features are similar to the design and approach of the $G^{0}$ experiment. 
A polarized electron beam was used and the helicity was selected every 20.08 ms by setting the high voltage of a fast Pockels cell according to a pattern of four helicity states $(+--+$ or -++-$)$. The experimental apparatus is shown in Fig. 3.5.

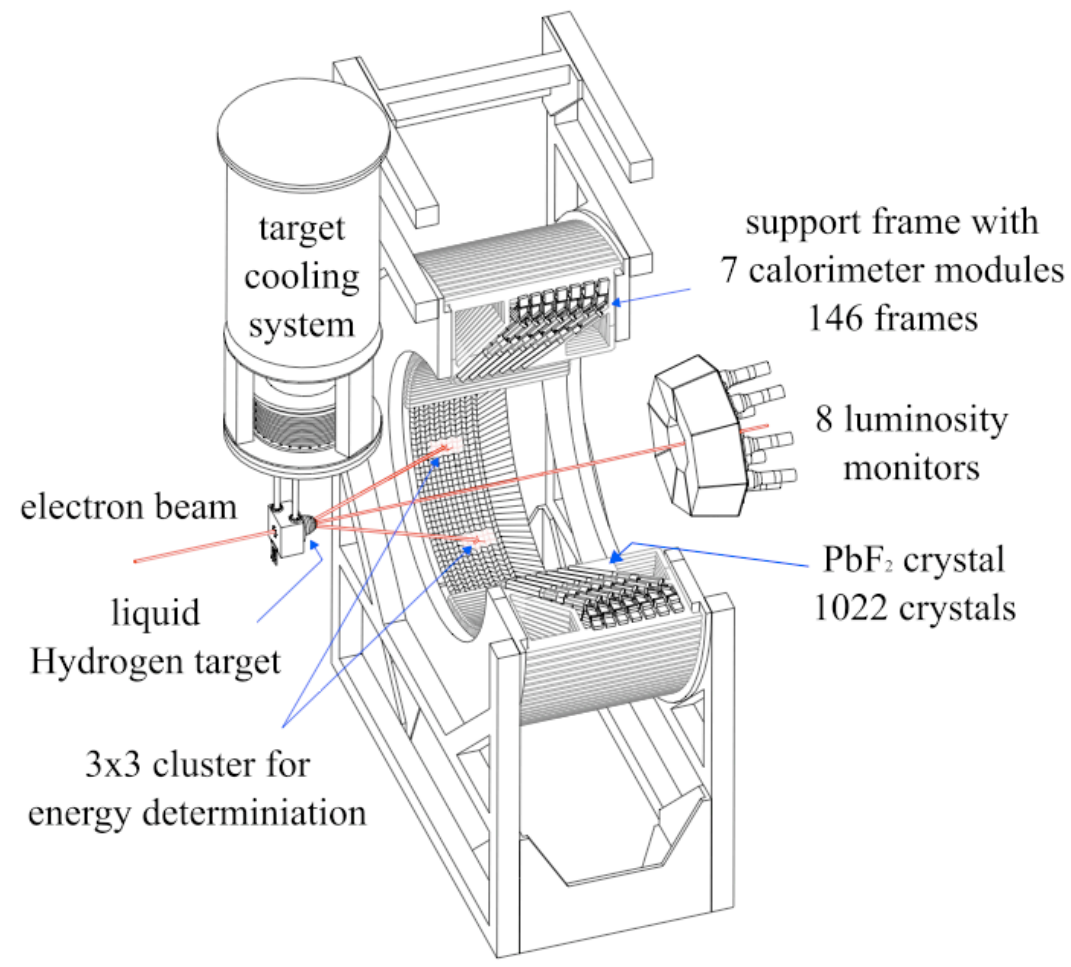

Figure 3.5: PVA4 target, calorimeter, and luminosity monitors [Rio09].

The scattered electrons were detected with a $\mathrm{PbF}_{2}$ Čerenkov shower calorimeter. The detector design included $1022 \mathrm{PbF}_{2}$ crystals arranged in seven rings, and processed in modules with self-triggering and histogramming electronics. The raw data for both the forward and backward angle running are shown in Fig. 3.6.

For the backward angle measurement, 72 plastic scintillators were installed in front of the $\mathrm{PbF}_{2}$ crystals (see Fig. 3.7). The scintillators were placed in two rings 

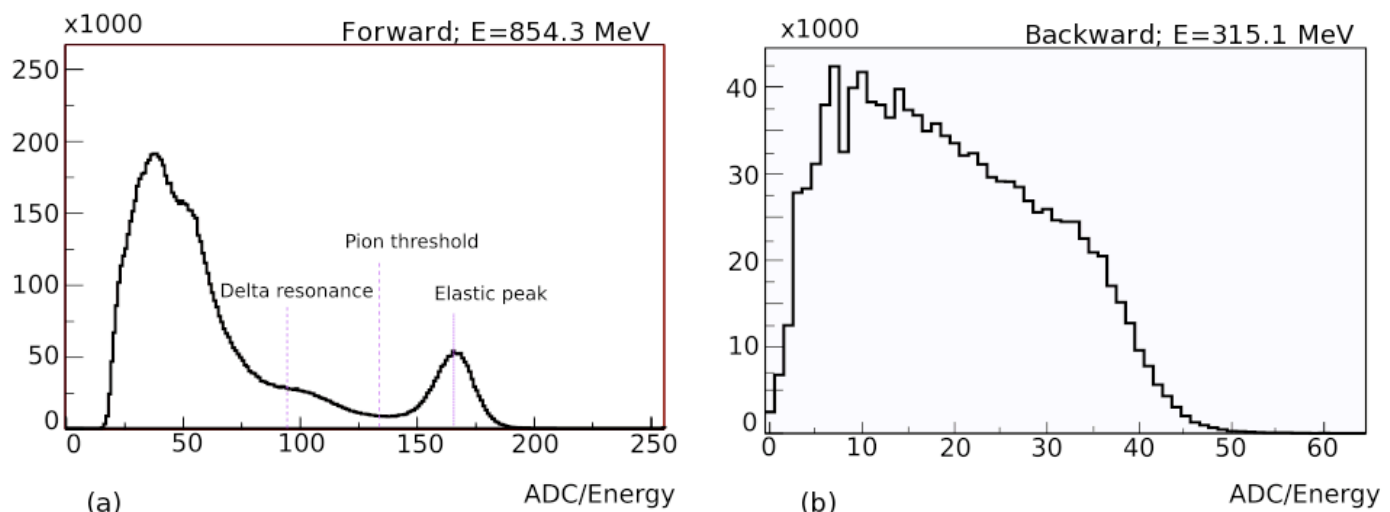

Figure 3.6: Data from PVA4. Forward angle data on the left (a) shows the elastic peak clearly and a distinct background dominated by $\pi^{\circ}$ and gammas. The data on the right from the backward angle running (b) shows the elastic electron peak at the same energy as $\pi^{\circ}$ and gamma background. [Rio09].

of 36 detectors per ring. Used in coincidence with the calorimeter, they enabled the separation of charged and neutral particles, and specifically, of scattered electrons from photons that were the result of $\pi^{\circ}$ decays. The energy deposited by a particle in the calorimeter was digitized by an ADC and stored in either a coincidence or a non-coincidence histogram depending on the scintillator trigger signal $\left[\mathrm{B}^{+} 09 \mathrm{a}\right]$.

The result of the addition of the scintillators can be seen in Fig. 3.8. The scintillators added an address-bit to the electronics allowing the electronics to produce four histograms every five minutes; one histogram of neutral particles and one of charged particles for each helicity state.

To date, three measurements have been taken using a $10 \mathrm{~cm}$ liquid hydrogen target; the first two at forward angles $\left(\approx 35^{\circ}\right)$, and one at a backward angle $(\approx$ $145^{\circ}$. To extract the vector strange form factors, the collaboration developed a new parameterization [Yak04] for the proton and neutron electromagnetic form factors that used a data set similar to the one selected by Kelly [Kel04]. 


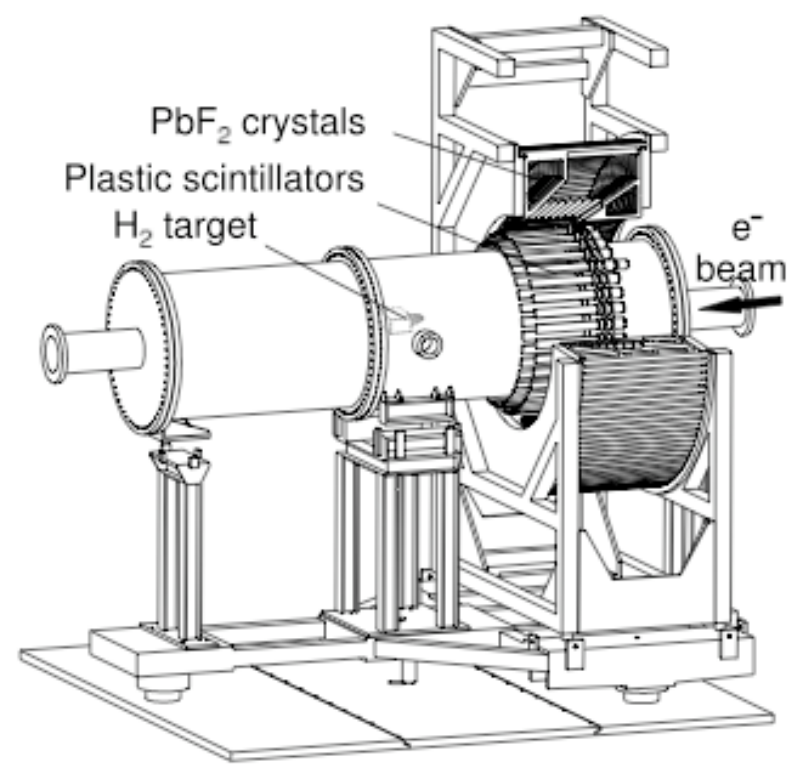

Figure 3.7: Drawing of the PVA4 $\mathrm{PbF}_{2}$ calorimeter in the backward angle configuration. The scintillators are placed between the scattering chamber and the lead fluoride crystals. [B $\left.B^{+} 09 a\right]$.

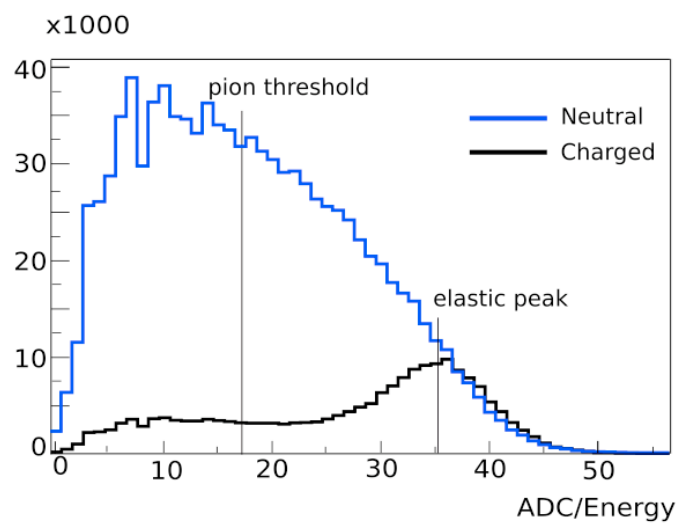

Figure 3.8: Separation of the elastic peak from the neutral particles through the use of scintillators that detect charged particles for the PVA4 experiment [Rio09]. 
The first measurement used a $20 \mu \mathrm{A}$ beam with an energy of $855 \mathrm{MeV}$ corresponding to $Q^{2}=-0.23(\mathrm{GeV} / \mathrm{c})^{2}$ where only 511 , or one half, of the channels of the detector and the readout electronics were operational. The measured asymmetry was $A_{p}=-5.44 \pm 0.54_{\text {(stat) }} \pm 0.26_{\text {(syst) }} \mathrm{ppm}\left[\mathrm{M}^{+} 04\right]$. The extracted value for the strange form factors was first reported in $\left[\mathrm{M}^{+} 04\right]$ with an updated value reported in $\left[\mathrm{B}^{+} 09 \mathrm{a}\right]$ as

$$
G_{E}^{s}+0.224 G_{M}^{s}=-0.020 \pm 0.029_{\text {stat }} \pm 0.016_{\text {syst }} .
$$

The second PVA4 measurement was also at forward angles and with $20 \mu \mathrm{A}$ of beam current, but the beam energy for this measurement was $570.4 \mathrm{MeV}$ with $Q^{2}=0.108$. The measured asymmetry was $A_{p}=-1.36 \pm 0.29_{(\text {stat })} \pm 0.13_{\text {(syst) }}$ ppm $\left[\mathrm{M}^{+} 05\right]$, with an extracted value for the strange form factor linear combination of

$$
G_{E}^{s}+0.106 G_{M}^{s}=0.071 \pm 0.036
$$

The third PVA4 measurement was at backward angles at $Q^{2}=0.22(\mathrm{GeV} / \mathrm{c})^{2}$, and a $20 \mu \mathrm{A}$ beam. The measured asymmetry was $A_{p}=-17.23 \pm 0.82_{\text {(stat) }} \pm 0.89_{\text {(syst) }}$ ppm $\left[\mathrm{B}^{+} 09 \mathrm{a}\right]$ and the extracted linear combination of the strange vector form factors was

$$
G_{E}^{s}+0.26 G_{M}^{s}=-0.12 \pm 0.11 \pm 0.11
$$

where the first error came from the measurement and the second from the uncertainty in the axial and electromagnetic form factors of the nucleon $\left[\mathrm{B}^{+} 09 \mathrm{a}\right]$. Combining this result with the PVA4 extracted values for the strange vector form factors obtained from the PVA4 forward angle measurement at $Q^{2}=0.23$, the collaboration 
was able to disentangle the form factors yielding the following results $\left[\mathrm{B}^{+} 09 \mathrm{a}\right]$ :

$$
\begin{gathered}
G_{E}^{s}=0.050 \pm 0.038 \pm 0.019 \\
G_{M}^{s}=-0.14 \pm 0.011 \pm 0.011 .
\end{gathered}
$$

\section{$3.4 G^{0}$ Forward Angle}

The $G^{0}$ forward angle measurement was taken in Hall C at Jefferson Laboratory from December 2003 through May 2004, and was the precursor to the backward angle measurement which is the subject of this thesis. The forward angle running used a $40 \mu \mathrm{A}$ polarized electron beam with an energy of $3.03 \mathrm{GeV}$. The beam structure incorporated a 32 ns timing pulse that allowed time-of-flight measurements for particle identification. The recoil protons were detected and sorted by $Q^{2}$, covering the range of $0.1<Q^{2}<1.0(\mathrm{GeV} / \mathrm{c})^{2}$. The experimental apparatus is described in Sec. 4.2 and is shown in Fig. 4.3 .

The forward angle results $\left[\mathrm{A}^{+} 05\right]$, shown in Fig. 3.9, extend the kinematic range and improve the precision of world parity-violating $e-p$ asymmetry measurements. The value of $G_{E}^{s}+\eta G_{M}^{s}$ uses the electromagnetic form factors of J.J. Kelly [Kel04]. Also shown is the excellent agreement with the HAPPEx-H results. The error bars include the statistical uncertainty (inner) and statistical plus point-topoint uncertainties (outer). The grey error bands represent the global systematic uncertainties $\left(G^{0}\right.$ only). The upper band shows the global uncertainties from the measurement and the lower band incorporates the theoretical uncertainties used to calculate $A_{N V S}$. Details of the measurement can be found in [Liu06] and [Nak06]. 


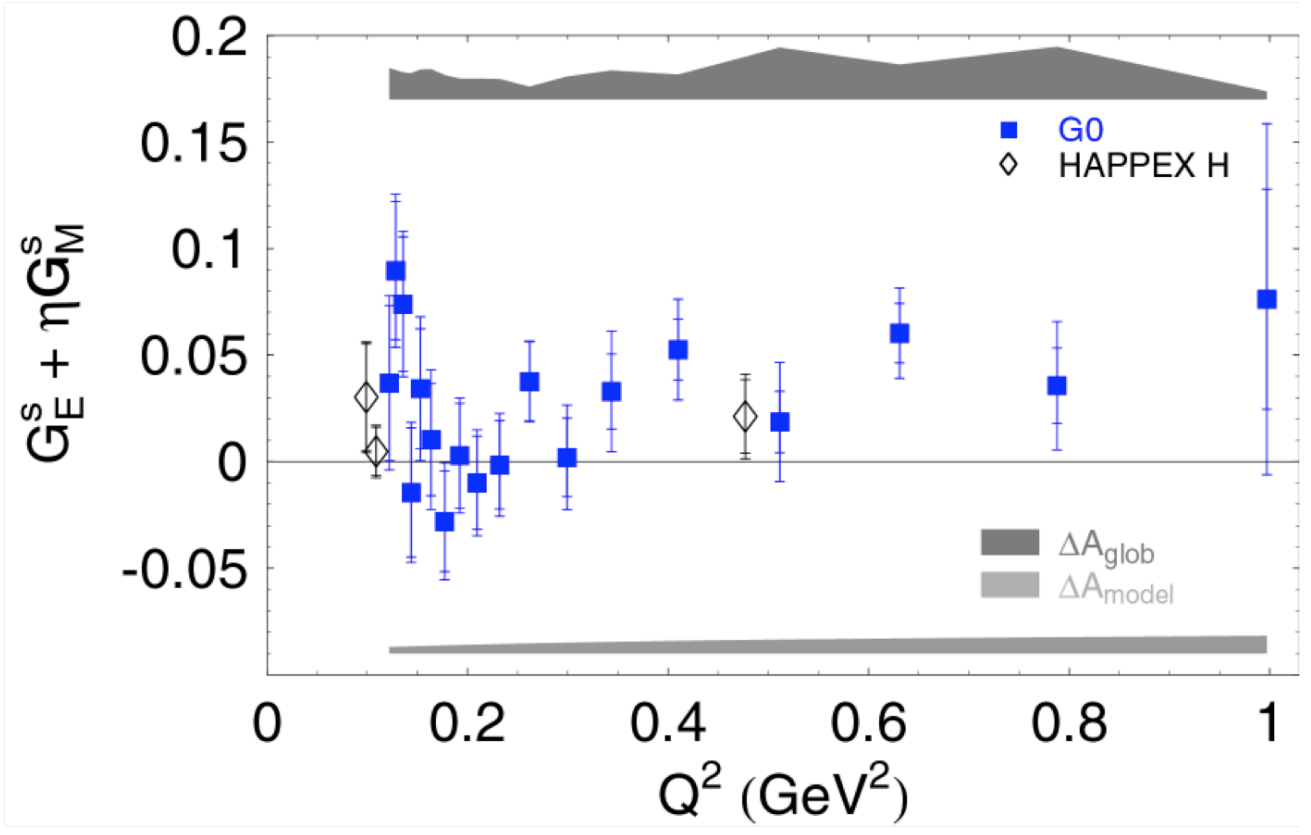

Figure 3.9: The results of the $G^{0}$ forward angle measurement as a function of fourmomentum transfer $\left[A^{+} 05\right]$. The error bars include the statistical uncertainty (inner) and statistical plus point-to-point uncertainties (outer). The grey error bands represent the global systematic uncertainties ( $G^{0}$ only). The upper band shows the global uncertainties from the measurement and the lower band incorporates the theoretical uncertainties used to calculate $A_{N V S}$. HAPPEx results $\left[A^{+} 06 c, A^{+} 06 b, A^{+} 07\right]$ are shown as diamonds for comparison. 


\subsection{Summary of Parity-Violating Measurements Prior to $G^{0}$ Back- ward Angle}

Table 3.1 provides a summary of world data from SAMPLE, HAPPEx-H, HAPPEx-He, PVA-4, and $G^{0}$ forward angle. SAMPLE II, SAMPLE III, and the third (backward angle) PVA-4 data are not included here because they are insensitive to $G_{E}^{s}$. The SAMPLE I result $\left[\mathrm{S}^{+} 04\right]$ was cast into $G_{E}^{s}+\eta G_{M}^{s}$ using a model calculation for $G_{A}^{e}[\operatorname{Liu06}]$.

Table 3.1: A summary of world data prior to the $G^{0}$ backard angle measurement for a linear combination of strange vector form factors, $G_{E}^{s}+\eta G_{M}^{s}$, and the associated kinematics. The first and second asymmetry uncertainties are statistical and systematic, respectively. The targets are hydrogen or helium $(\eta=0)$.

\begin{tabular}{cccccc}
\hline \hline $\begin{array}{c}Q^{2} \\
(G e V / c)^{2}\end{array}$ & $\begin{array}{c}\theta_{\text {lab }} \\
( \pm 10 \%)\end{array}$ & $A_{\text {phys }}$ & $\begin{array}{c}\eta \\
(\mathrm{ppm})\end{array}$ & $G_{E}^{s}+\eta G_{M}^{s}$ & Ref \\
\hline \hline 0.091 & 145 & $-5.61 \pm 0.67 \pm 0.88$ & 1.67 & $0.56 \pm 0.55$ & {$[\mathrm{Liu} 06]$} \\
0.477 & 12 & $-15.05 \pm 0.98 \pm 0.56$ & 0.392 & $0.014 \pm 0.22$ & {$\left[\mathrm{~A}^{+} 04\right]$} \\
0.077 & 6 & $6.40 \pm 0.23 \pm 0.12$ & 0 & $0.002 \pm 0.016$ & {$\left[\mathrm{~A}^{+} 07\right]$} \\
0.109 & 6 & $-1.58 \pm 0.12 \pm 0.04$ & 0.09 & $0.007 \pm 0.013$ & {$\left[\mathrm{~A}^{+} 07\right]$} \\
0.23 & 35 & $-5.44 \pm 0.54 \pm 0.26$ & 0.224 & $0.020 \pm 0.033$ & {$\left[\mathrm{~B}^{+} 09 \mathrm{a}\right]$} \\
0.108 & 35 & $-1.36 \pm 0.19 \pm 0.13$ & 0.106 & $0.071 \pm 0.036$ & {$\left[\mathrm{M}^{+} 05\right]$} \\
0.122 & 13 & $-1.51 \pm 0.44 \pm 0.28$ & 0.098 & $0.037 \pm 0.043$ & {$\left[\mathrm{~A}^{+} 05\right]$} \\
0.232 & 13 & $-5.27 \pm 0.51 \pm 0.38$ & 0.189 & $-0.002 \pm 0.026$ & {$\left[\mathrm{~A}^{+} 05\right]$} \\
0.410 & 13 & $-10.25 \pm 0.67 \pm 1.05$ & 0.341 & $0.053 \pm 0.027$ & {$\left[\mathrm{~A}^{+} 05\right]$} \\
0.631 & 13 & $-19.96 \pm 1.11 \pm 1.83$ & 0.543 & $0.060 \pm 0.028$ & {$\left[\mathrm{~A}^{+} 05\right]$} \\
0.997 & 13 & $-37.93 \pm 7.24 \pm 9.02$ & 0.932 & $0.076 \pm 0.083$ & {$\left[\mathrm{~A}^{+} 05\right]$} \\
\hline \hline
\end{tabular}

It should be noted that the values obtained by each collaboration for $G_{E}^{s}+\eta G_{M}^{s}$ and shown in Table 3.1, have used different parameterizations for the nucleon electromagnetic form factors and for the electroweak radiative corrections. Because of 
this, it is worthwhile to consider a global analysis of all of the world data, using common electromagnetic form factors, and common electroweak radiative corrections parameters.

Figure 3.10 shows a fit for all of the world data collected prior to the $G^{0}$ backward angle measurement [LMRM07]. The yellow and gray blue ellipses represent the 1- $\sigma$ and 2- $\sigma$ confidence contours around the point of maximum likelihood at $G_{E}^{s}=-0.004, G_{M}^{s}=0.30$

The $G^{0}$ backward angle measurements taken at $Q^{2}=0.22(\mathrm{GeV} / \mathrm{c})^{2}$ and $Q^{2}=0.63(\mathrm{GeV} / \mathrm{c})^{2}$ expand the kinematic range of world data and allow the first experimental separation of $G_{E}^{s}$ and $G_{M}^{s}$ at $Q^{2}>0.1(\mathrm{GeV} / \mathrm{c})^{2}$.

The $G^{0}$ experimental apparatus, with an emphasis on the backward angle configuration, is discussed in the following chapters. The $G^{0}$ backward angle data analysis follows in Section, 5.7, and the final $G^{0}$ results are presented in Section 6.4. 


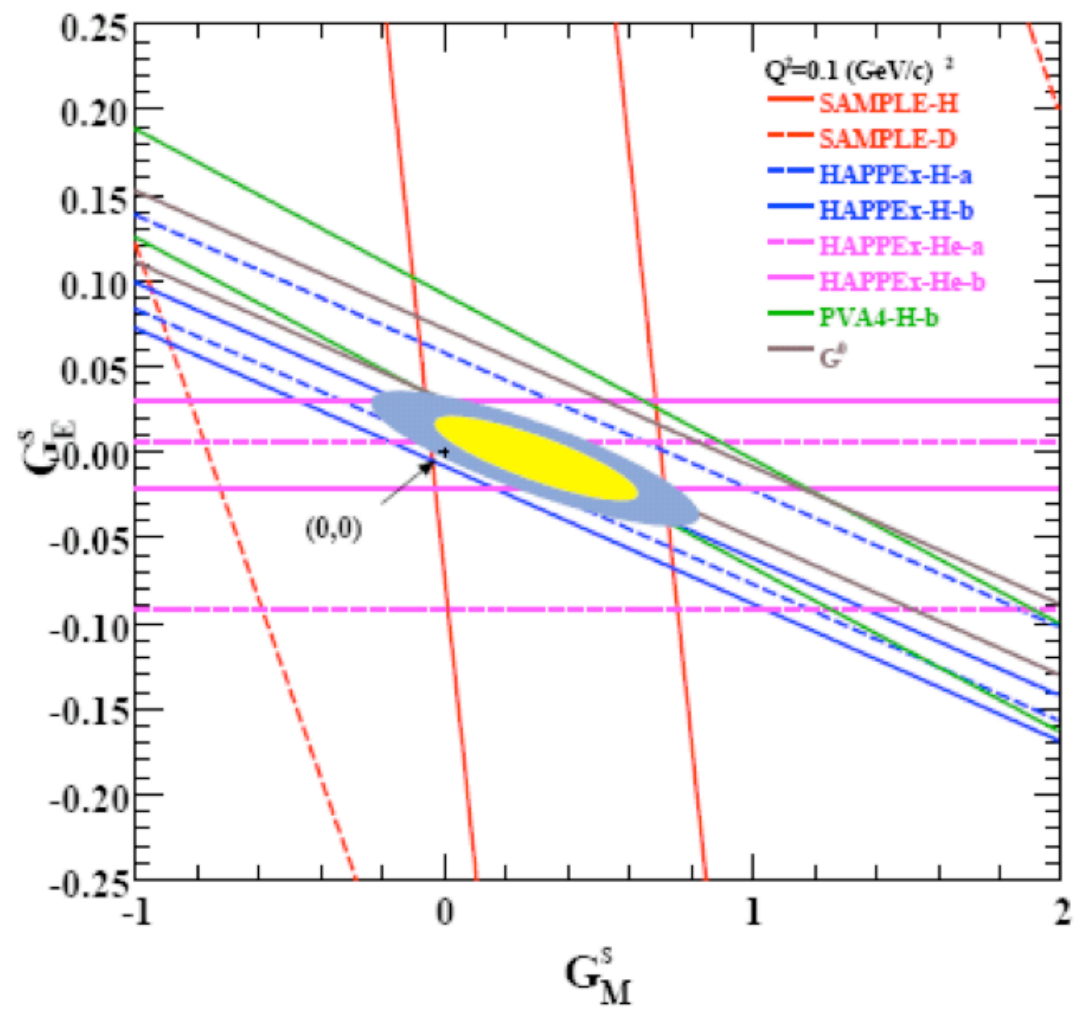

Figure 3.10: World data constraints on $\left(G_{E}^{s}, G_{M}^{s}\right)$ at $Q^{2}=0.1(G e V / c)^{2}$. The Kelly form factors [Kel04] are used. Different bands in the plot represent: SAMPLE-H [S ${ }^{+}$00] (solid red), SAMPLE-D [I+ 04] (dashed red), HAPPEx-H-a [A+99b] (dashed blue), Happex-H-b [A+01] (solid blue), Happex-he-a [A+06b] (dashed pink), Happexhe-b [A+07] (solid pink), PVA4-H [M+05] (solid green), and $G^{0}$ forward angle $\left[A^{+} 05\right]$ (solid brown). The yellow and grey ellipses represents $68.27 \%\left(\Delta \chi^{2}=2.3\right)$ and $95 \%\left(\Delta \chi^{2}=5.99\right)$ confidence contours around the point of maximum likelihood at $G_{E}^{s}=-0.004, G_{M}^{s}=0.30$. The black cross represents $G_{E}^{s}=G_{M}^{s}=0$. [LMRMO'7]. 


\section{Chapter 4}

\section{The $G^{0}$ Backward Angle Experimental Apparatus}

\subsection{Overview}

The $G^{0}$ backward angle experiment was designed to measure the parity-violating asymmetry seen when polarized electrons were elastically (quasi-elastically) scattered from an unpolarized hydrogen (deuterium) target. Eqn. 2.48 in Sec. 2.2.1 expressed the parity-violating asymmetry as the difference in the cross section of the two helicity states, divided by their sum. Because the detector yield normalized to the beam current is proportional to the cross section, the experimental technique is to flip the helicity of the electron beam, between positive and negative helicity states, and measure the normalized detector yield for each helicity state. Therefore, the parity-violating asymmetry can be expressed as

$$
A_{p v}=\frac{Y_{+}-Y_{-}}{Y_{+}+Y_{-}}
$$

where $Y_{+(-)}$is the normalized yield for the positive(negative) helicity state.

Because the parity-violating neutral weak interaction is suppressed in comparison to the parity-conserving electromagnetic interaction, the size of the asymmetry is expected to be of order 10 parts per million (ppm). The small size of the expected asymmetry increased the challenge of this experiment in terms of both the required precision in the measurement and the length of the experimental run time. In or- 
der to ensure a statistical uncertainty of $5 \%$, approximately $10^{14}$ scattering events were required for each beam energy. Precise knowledge of beam properties were crucial because even small, systematic effects could have a large relative impact on the asymmetry result. Measurements were taken at two different beam energies, 362 $\mathrm{MeV}$ and $687 \mathrm{MeV}$, roughly equating to $Q^{2}=0.23(\mathrm{GeV} / \mathrm{c})^{2}$ and $Q^{2}=0.61(\mathrm{GeV} / \mathrm{c})^{2}$ respectively, with each of the liquid targets (hydrogen and deuterium).

The $G^{0}$ backward angle spectrometer was comprised of a toroidal superconducting magnet system (SMS) and three detector arrays mounted on a support structure referred to as the ferris wheel, as shown in Fig. 4.1.

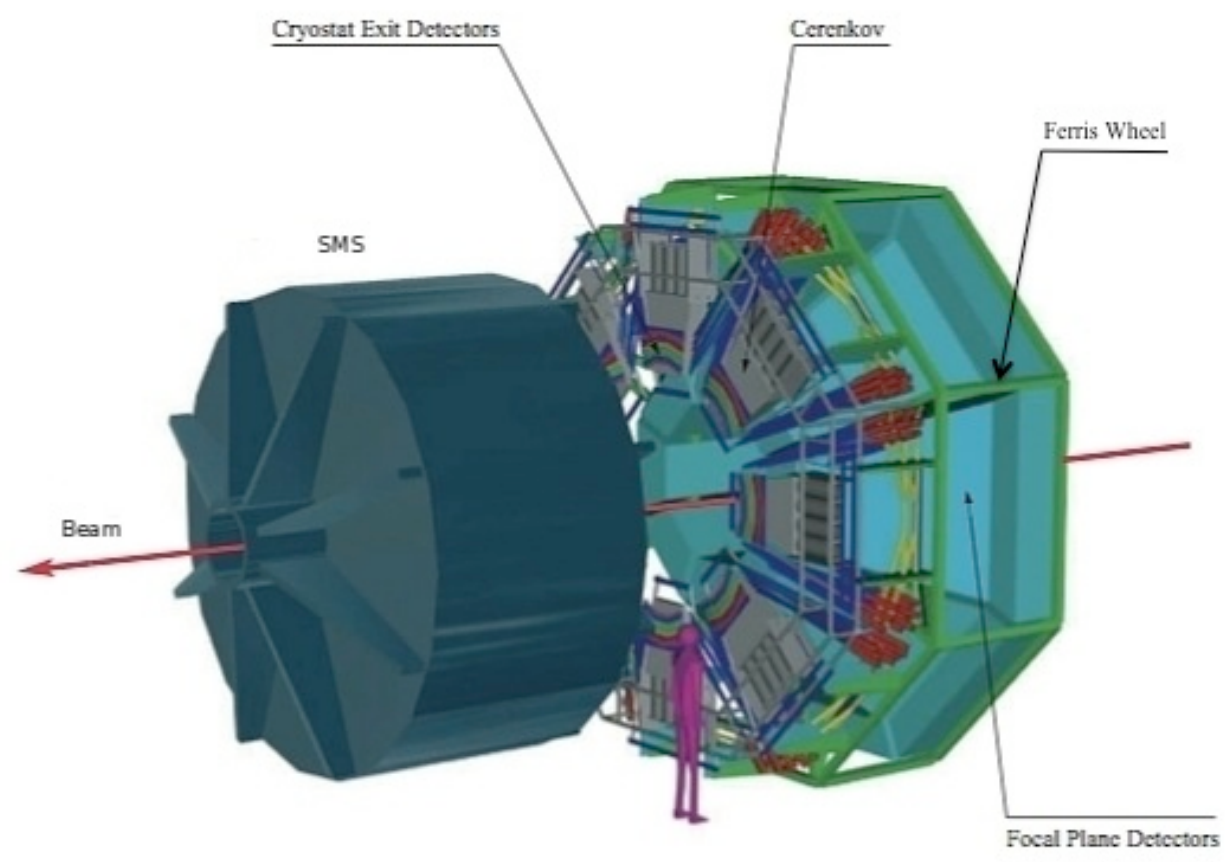

Figure 4.1: A schematic drawing of the $G^{0}$ spectrometer including the SMS and detectors mounted on the ferris wheel.

The SMS focused the elastically and quasi-elastically scattered electrons into the detectors at an electron scattering angle of $\approx 108^{\circ}$ defined by the target loca- 
tion, the lead collimators shown in Fig. 4.2 and the SMS magnetic field. In order to separate scattered electrons from background particles, such as negatively-charged pions $\left(\pi^{-}\right)$, aerogel Čerenkov detectors were used. The separation of inelastic electrons from elastic or quasi-elastic events was accomplished using coarse momentum and scattering angle information provided by two sets of detectors, the Focal Plane Detectors (FPDs) and the Cryostat Exit Detectors (CEDs). The spectrometer

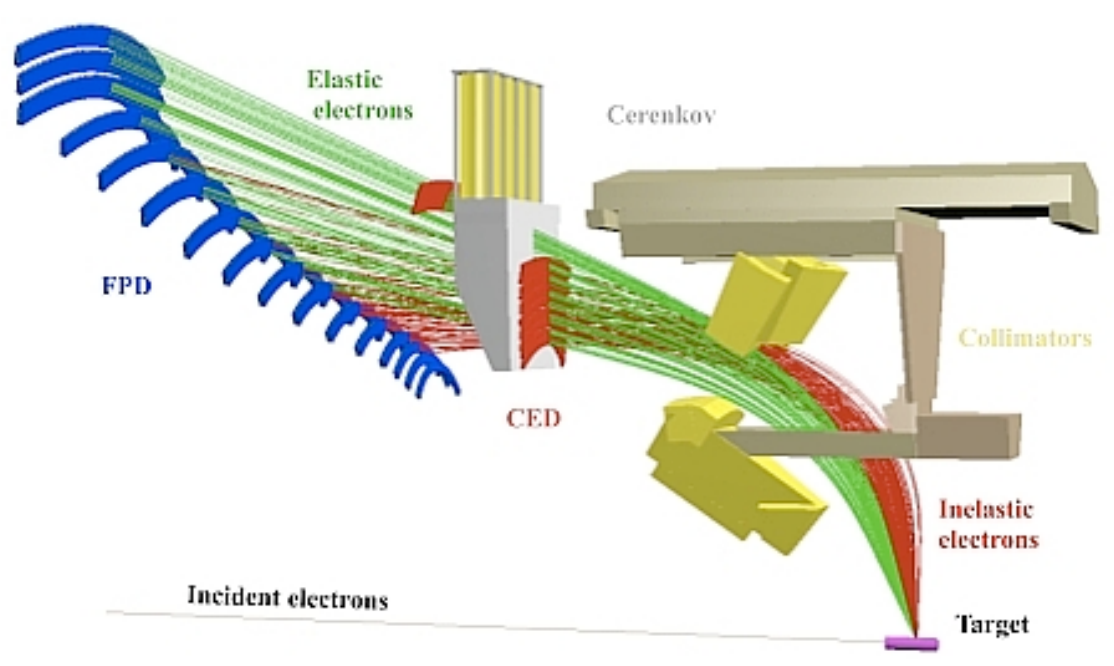

Figure 4.2: A schematic drawing of the particle trajectory from the target to the detectors in the $G^{0}$ spectrometer for the backward angle measurement. Only one of the eight detector sets is depicted.

was constructed in eight sections, or octants, with each octant having its own set of SMS collimators and detector arrays, as shown in Fig. 4.2. Four of the eight octants (numbered 1, 3, 5, and 7) were assembled by a North American (NA) collaboration [gC98] and four $(2,4,6$, and 8) by a French collaboration [gC98]. When looking down the beam line toward the spectrometer, octant one was located at the 12 o'clock position and the rest of the octants were numbered moving counter-clockwise. 


\subsection{Forward Angle Apparatus}

For complete separation of $G_{E}^{s}, G_{M}^{s}$, and $G_{A}^{e}$, the results from the backward angle measurement were combined with the forward angle measurement $\left[\mathrm{A}^{+} 05\right]$ as discussed in Sec. 2.4 and Sec. 2.2.1. To optimize the experimental separation of $G_{E}^{s}$ and $G_{M}^{s}$, the forward angle measurement was run at a small electron scattering angle, and the backward measurement at a large electron angle. The $G^{0}$ forward

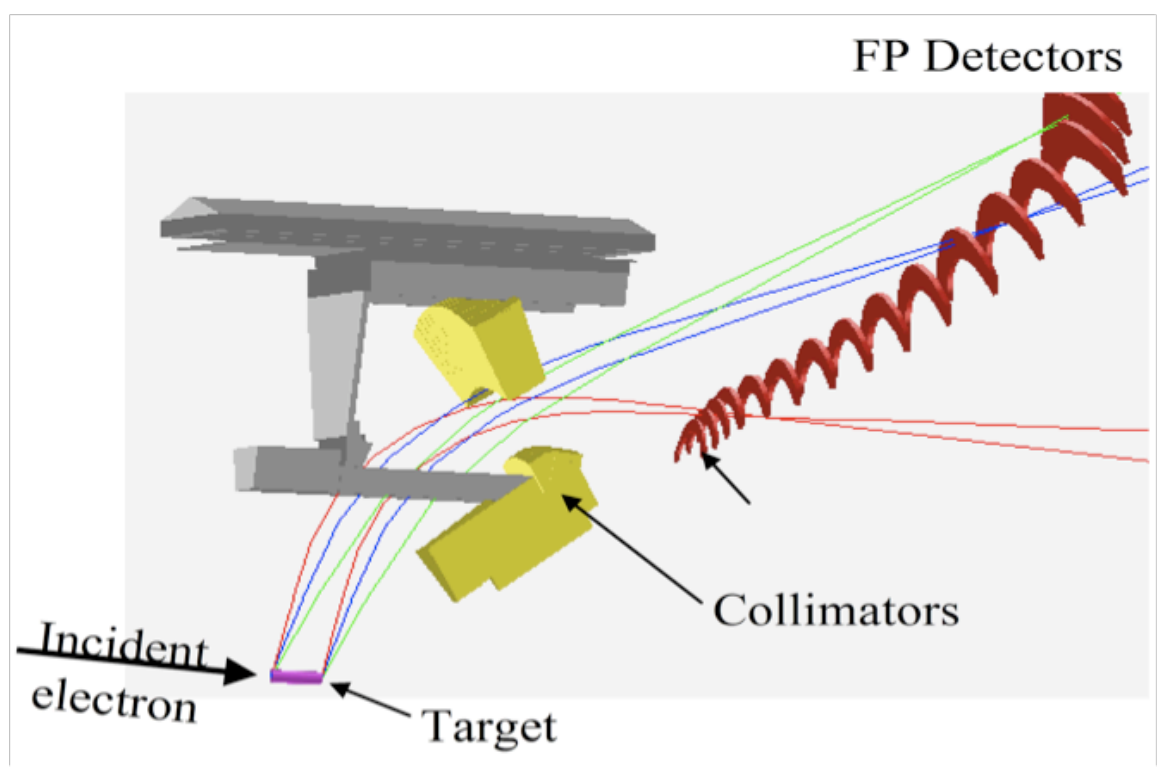

Figure 4.3: A schematic of the particle trajectory from the target to the detectors in the $G^{0}$ spectrometer for the forward angle measurement. Only one of the eight detector sets is depicted.

angle experiment was designed not only to detect recoil protons, but also so that the majority of the hardware could be re-used for the backward angle measurement. The $G^{0}$ target and SMS were used for both measurements. The SMS was turned $180^{\circ}$ and physically relocated to the other side of the target for the backangle running. Additionally, the FPDs were reused, with minor modifications (discussed in Sec. 4.4). For the forward angle measurement, the FPDs consisted of 16 scintillator 
detectors placed on the focal surface of the magnet. Each detector's scintillator detected recoiled protons at a unique $Q^{2}$. A schematic drawing of one octant of the forward angle spectrometer and target is shown in Fig. 4.3.

The forward angle measurement was made at a beam energy of $3.031 \mathrm{GeV}$. The four-momentum transfer ranged from $0.12<Q^{2}<1.0(\mathrm{GeV} / \mathrm{c})^{2}\left[\mathrm{~A}^{+} 05\right]$. Time of flight measurements were used to separate elastic proton recoil events from background particles and inelastic events. The electronics was completely redesigned for the backward angle measurement and the data acquisition system (DAQ) was greatly modified because separation of background particles $\left(\pi^{-}\right.$inelastic $\left.\mathrm{e}^{-}\right)$from the elastic or quasi-elastic electrons using time of flight was not feasible.

\subsection{Superconducting Magnet System}

The SMS focused scattered charged particles possessing the same momentum and scattering angle, originating from anywhere along the $20 \mathrm{~cm}$ length of target, to a single point in one of the eight octants of the detector array. It was constructed by BWX Technologies, Inc $(B W X T)^{1}$, modified by the University of Illinois, UrbanaChampaign, and installed in Hall $\mathrm{C}$ at Jefferson Lab. It was an iron-free toroidal magnet comprised of eight superconducting coils in a single cryostat. The coils were arranged azimuthally around a central core region with periodic gaps between the windings, as shown in Fig. 4.4. The gaps allowed the scattered electrons to pass between collimators within the SMS on their way to the detector arrays. The coils

\footnotetext{
${ }^{1}$ BWXT is now a group of The Babcock and Wilcox companies headquartered in Lynchburg,
} VA. 
were Niobium-Titanium and became superconducting, cooled by liquid helium, at temperatures below $8 \mathrm{~K}$ with a nominal operating temperature of $4.5 \mathrm{~K}$. They

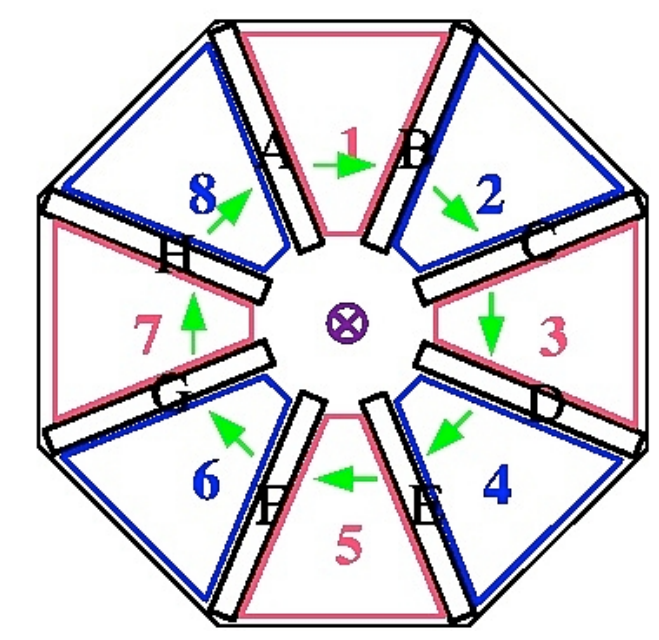

Figure 4.4: A schematic drawing of the eight superconducting coils viewed from the target and looking upstream (backward angle mode). The magnetic field direction is shown by the green arrows. The layout of the coils, (A-H) and the detector octants (1-8) is shown. The beam is located at the cross in the center.

were enclosed in a liquid nitrogen shield maintained at a temperature of $110 \mathrm{~K}$. The maximum coil current was 5000 A, or a magnetic field integral of 1.6 Tesla-meters [Nak06]. The nominal coil current for the $G^{0}$ backward angle measurement was $3500 \mathrm{~A}$ when the beam energy was $687 \mathrm{MeV}$, and $2650 \mathrm{~A}$ when the beam energy was $362 \mathrm{MeV}$.

A toroidal spectrometer was selected because of its large acceptance, its asymuthal symmetry, and because the magnetic field is negligible near the central axis where the target is located. Also, the toroidal design could handle both the forward angle measurement's recoil protons ( $\approx 65^{\circ}$ scattering angle) and the backward angle measurement's scattered electrons $\left(\approx 108^{\circ}\right)$ by simply moving the magnet to the other side of the target and adjusting the coil current to the appropriate setting to 
match the energy of the beam.

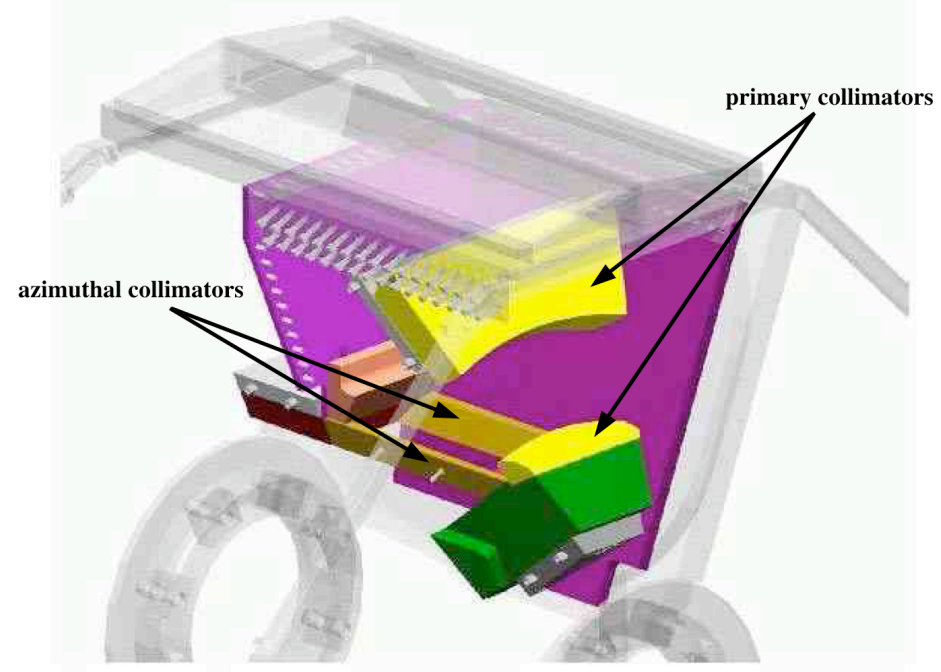

Figure 4.5: SMS collimators shown for one octant [Bat04]

Lead collimators defined the acceptance of the scattered particles and provided shielding against gammas by blocking the direct view between the target and the detector arrays. There were two sets of lead collimators as shown in Fig. 4.5. The upstream set were azimuthal collimators with a $\pm 10^{\circ}$ opening in order to cut off the acceptance close to the coils thereby ensuring a uniform magnetic field for all detected particles. The down stream collimators shielded the detectors from the direct view of the target and limited the range of electron recoil angles seen in the detectors. 


\subsection{Focal Plane Detectors}

The FPD array for the backward angle measurement consisted of 14 pairs of arc-shaped plastic scintillators per octant. Each scintillator had two lucite light guides, one light guide attached to each end of the scintillator. At the end of each light guide was a photomultiplier tube, as shown in Fig. 4.6. The light-guides

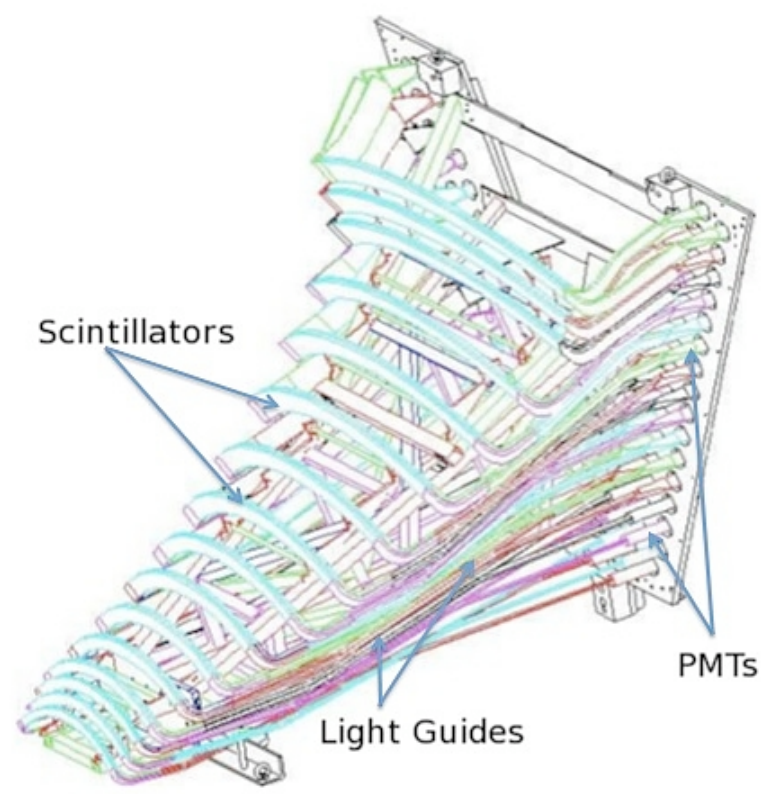

Figure 4.6: Focal Plane Detectors (FPDs) for one octant.

were necessary to position the photomultiplier tubes in a low magnetic field region.

For the French octants, 8-stage Photonis XP2282 photomultiplier tubes were used with custom-made bases. The NA octants used 12-stage Phillips XP-2262B photomultiplier tubes with custom-built passive bases with Zener diodes on the first stage to stabilize the collection efficiency. The NA design also used Zener diodes on the stages before the anode to limit instabilities due to high rates. Photomultiplier tube life-time was a concern so the tubes were run at low gain and the signals were 
amplified. The French bases included a pre-amplifier providing an amplification factor of 20 and the NA bases used modified commercial Phillips 776 amplifiers to achieve an amplification factor of $25\left[\mathrm{M}^{+} 08\right]$.

The scintillator pairs were numbered $3-16$, with 3 being the scintillator pair with the shortest length and which was closest to the target. The forward angle measurement used 16 pairs but for the backward angle measurement, scintillator pairs 1 and 2 were removed. Each scintillator pair was comprised of two identically shaped scintillators, lying back to back with one another, constructed of Bicron BC408. The scintillator thickness varied for the low numbered pairs to accommodate the low proton energies in these detectors for the forward angle measurment. Each scintillator in detector pair 3 was $0.5 \mathrm{~cm}$ thick. For detector pair 4 , the front scintillator had a thickness of $0.5 \mathrm{~cm}$, while the back scintillator was $1 \mathrm{~cm}$ thick. Scintillators 5-16 each were $1 \mathrm{~cm}$ thick [Ver09].

The scintillators were polished and wrapped in aluminized mylar (NA) or aluminum foil (French). Each pair of French FPD scintillators was separated by $3 \mathrm{~mm}$ of aluminum while the NA pairs were separated by $3 \mathrm{~mm}$ of polycarbonate $\left[\mathrm{M}^{+} 08\right]$. This was done for the forward angle measurement in order to reduce the background from neutral particles in the front scintillator generating charged particles that then would trigger the back scintillator. During the forward angle measurement one of the requirements for a "good hit" in an FPD was a coincidence between the front and back scintillator. For the backward angle measurement, this was initially thought to be unnecessary, so the requirement for a front-back coincidence was removed and all of the photomultiplier tubes associated with the "back" scintillator were installed 
in the CED detector array. Once the experiment began running with the deuterium target, high background rates were seen. The collaboration decided to take one of the "front" FPD photomultiplier tubes and move it to one of the "back" positions and then once again require a front-back coincidence. This adjustment reduced the number of neutral background particles triggering the FPDs.

\subsection{Cryostat Exit Detectors}

As mentioned previously, the CEDs in coincidence with the FPDs provided coarse momentum and scattering angle information. The CED arrays were built by the NA collaboration using the photomultiplier tubes and bases that had been used in the "back" FPD position during the forward angle measurement, with the caveat that French photomultiplier tubes and bases were used in French octants and NA detectors in NA octants.

Each octant contained nine CEDs, with CED 1-8 positioned just downstream from the aerogel Čerenkov detectors and just upstream of the SMS cryostat exit windows. CED-9 was positioned just upstream of the Čerenkov detectors. The CEDs were constructed in a similar manner as one layer (front or back) of FPDs. Each CED had one BC-408 scintillator. Attached to each end of the scintillator were light guides and attached to each end of the light guides were photomultiplier tubes. The CEDs were mounted in a separate frame for each octant as shown in Fig. 4.7. Each CED frame was attached to the outer ring of the ferris wheel, which is shown in Fig. 4.1. 


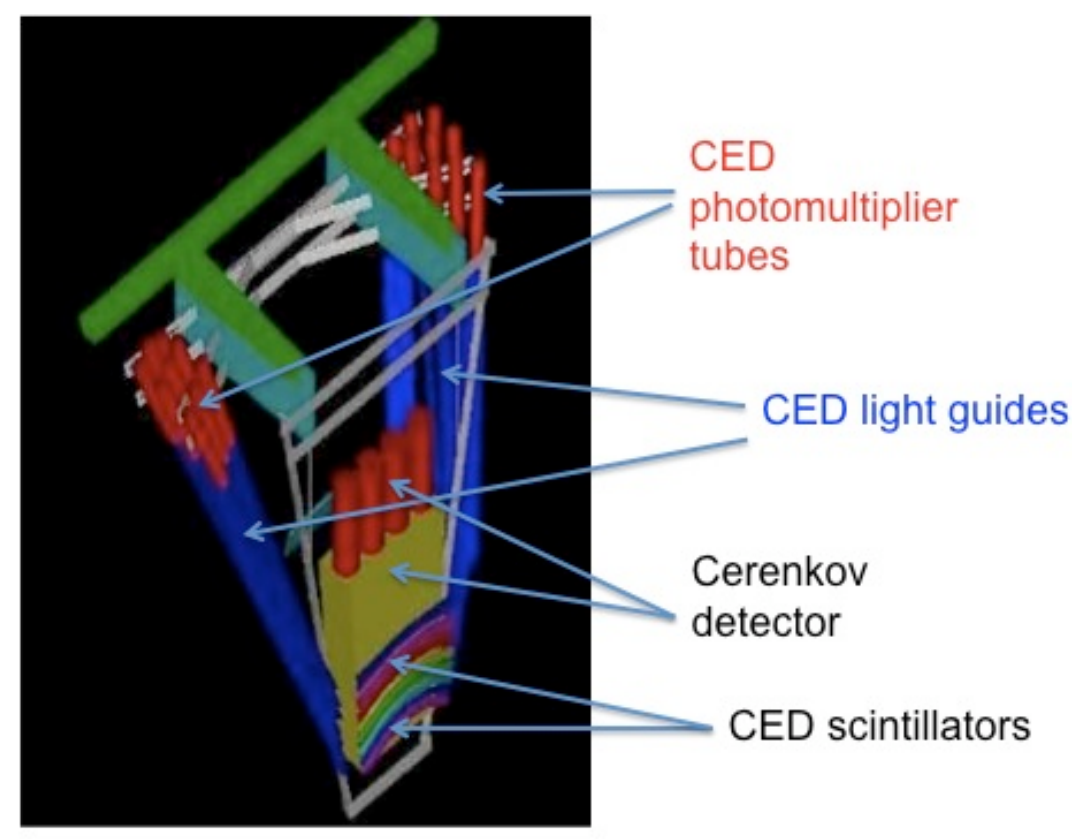

Figure 4.7: Cryotstat Exit Detector (CED) and support structure for one octant.

\section{6 Čerenkov Detectors}

Negatively charged pions, $\pi^{-}$, could produce a significant background to the elastic and quasi-elastic scattering rates for both targets during the backward angle measurement, and particularly for high energy scattering from the deuterium target. The $\pi^{-}$were generated from electron interactions with the aluminum target walls and from delta-resonance decay excited from electron-neutron interactions in the deuterium target. Silicon aerogel Čerenkov detectors with a refractive index of $\mathrm{n}=1.03$ were used to detect scattered electrons and thereby provides a means to discriminate between electrons and pions. Each detector contained five layers of 1 cm thick aerogel tiles, each tile had a volume of $\approx 11 \times 11 \times 1 \mathrm{~cm}^{3}$, and each layer contained approximately 30 tiles [Lee06]. Attached to the light diffusion box, were four photomultiplier tubes, which were initially 5 inch Photonis XP-4572, Fig. 4.8. 


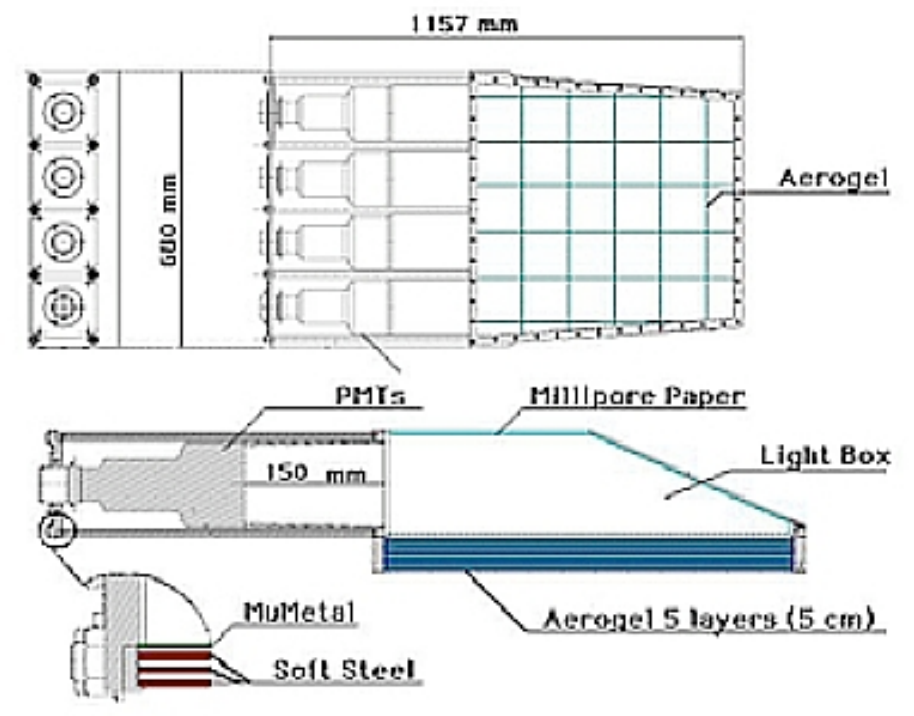

Figure 4.8: Čerenkov design including magnetic shielding [Gui05]

The initial runs for the backward angle measurement used the hydrogen target. Once the target was switched to deuterium, rates five times higher than during the hydrogen running were seen in the Čerenkov detectors. The count rates were so high that the beam current had to be reduced from $60 \mu \mathrm{A}$ to $10 \mu \mathrm{A}$ to avoid unacceptably high anode currents that could damage the photomultiplier tubes. Initial troubleshooting verified that $80 \%$ of the count rate originated in the photomultiplier tubes themselves [BEG06] and neutron capture in the photomultiplier tube's borosilicate glass was suspected as the cause. It was hypothesized and then corroborated using simulation, that neutrons were generated possessing a few $\mathrm{MeV}$ of energy and were then slowed to the eV range in the floor, walls, and other shielding prior to reaching the Čerenkov photomultiplier tubes.

Boron-10 $\left({ }^{10} \mathrm{~B}\right)$, a component in the borosilicate glass face and envelope of the 
photomultiplier tubes, has a very high cross section for thermal neutron capture and $20 \%$ of naturally occurring boron is ${ }^{10} \mathrm{~B}$. When a thermal neutron is captured, the following reaction occurs $94 \%$ of the time:

$$
{ }^{10} \mathrm{~B}+\mathrm{n} \rightarrow \alpha+{ }^{7} \mathrm{Li}+\gamma[0.48 \mathrm{MeV}]
$$

The decay products produce scintillation light in the glass, releasing photoelectrons in the active photocathode on the front window face of the photomultiplier tube. Testing was conducted at the National Institute of Standards and Technology, Gaithersburg Maryland using a mono-energetic, collimated, cold neutron beam (3.3 meV, $4.95 \AA$ ) [BEG06]. The testing showed that for each neutron absorbed in the photomultiplier tube's borosilicate front window, as many as four photoelectrons were produced, spread over several $\mu$ secs. The time period was large enough so that an average of 3.5 time-separated events per neutron were recorded. The testing also determined that 5 to $20 \%$ of the rate was coming from capture on the photomultiplier tube's glass envelope. The tests indicated that replacing the borosilicate glass window with a quartz window could substantially reduce the rate [BEG06]. Because of these results, all of the borosilicate Čerenkov photomultiplier tubes were replaced with Photonix XP4578 tubes which have a quartz face and borosilicate envelopes. ${ }^{2}$ There was a $50 \%$ reduction in the rate using the new photomultiplier tubes with the quartz faces. The final result of the change in tubes was a significant improvement in the number of events obtained for the same amount of run time, as the new tubes allowed the beam current to be increased to $35 \mu \mathrm{A}$ for the low energy deuterium

\footnotetext{
${ }^{2}$ There were no manufacturers producing photomultiplier tubes with quartz envelopes.
} 
runs, and $20 \mu \mathrm{A}$ for the high energy deuterium runs.

The NA Čerenkov detectors used a Photonis VD105K passive, non-Zener, negative high voltage base and a custom pre-amplifier that boosted the anode output signal. The French detectors used a similar base that was custom built, which also included a pre-amplifier within the base. A low voltage power supply was required for the post-amplifiers for both the NA and French designs.

The Čerenkov detectors were located adjacent to, and just upstream of CEDs 1-8, as shown in Figs. 4.1, 4.2, and 4.7. There was one Čerenkov detector per octant with each detector having four photomultiplier tubes, as shown in Fig. 4.8. The CED frame and detectors provided the support for the Čerenkov detectors (see Fig. 4.7).

\subsection{Electronics}

The $G^{0}$ backangle measurement used electronics that allowed individual events to be counted, rather than relying on an integrated signal. The electronics were designed to not only count particles striking individual detectors but also to identify individual particles as either electrons or pions and then, if the particle was an electron, to determine whether the scattering was elastic (quasi-elastic) or inelastic. This was accomplished by recording the count rate in all of the detectors using counting scalers as well as by determining when and in which detectors a three-fold (CED, FPD, and Čerenkov) or a two-fold (CED and FPD) coincidence occurred. Half of the electronics were designed and built by the NA collaboration to record 
data seen in the detectors in the octants that were constructed in North America, and the other half was designed and built by the French collaboration. Although the goal was the same, there are differences in the way each collaboration's electronics accomplished the task. Having two highly specialized sets of electronics with associated detectors designed and built by two independent groups, provided an outstanding cross-check of systematics associated with the electronics.

Despite the differences, the basic approach was the same and is described here. When a photomultiplier tube was fired in either an FPD or a CED, the signal was sent to a constant fraction discriminator (CFD) that selected signals that were at or above a set signal amplitude threshold. This threshold was about $50 \%$ of the amplitude for normal incident high energy electrons. This eliminated errant signals produced by thermal noise or low energy random particles. When both photomultiplier tubes were fired in a single detector (either FPD or CED), the CFD signals from both ends of the detector were combined together in a Mean Timer, that produced a signal based on the average time of the two scintillator hits. This ensured that the timing of the event was independent of the hit location along the scintillator. Basic schematics of the two approaches are shown in Fig. 4.9 (NA) and Fig. 4.10 (French). When at least one CED or one FPD had a mean timed signal, a trigger signal was generated. The electronics logic would check for hits in the CEDs and the FPDs in the proper timing window of the trigger. If at least one CED and one FPD were hit within the proper timing window, a coincidence was recorded. If more than one CED or more than one FPD was hit when a coincidence was recorded, the event would be recorded as a "multi-hit". For the Cerenkov 


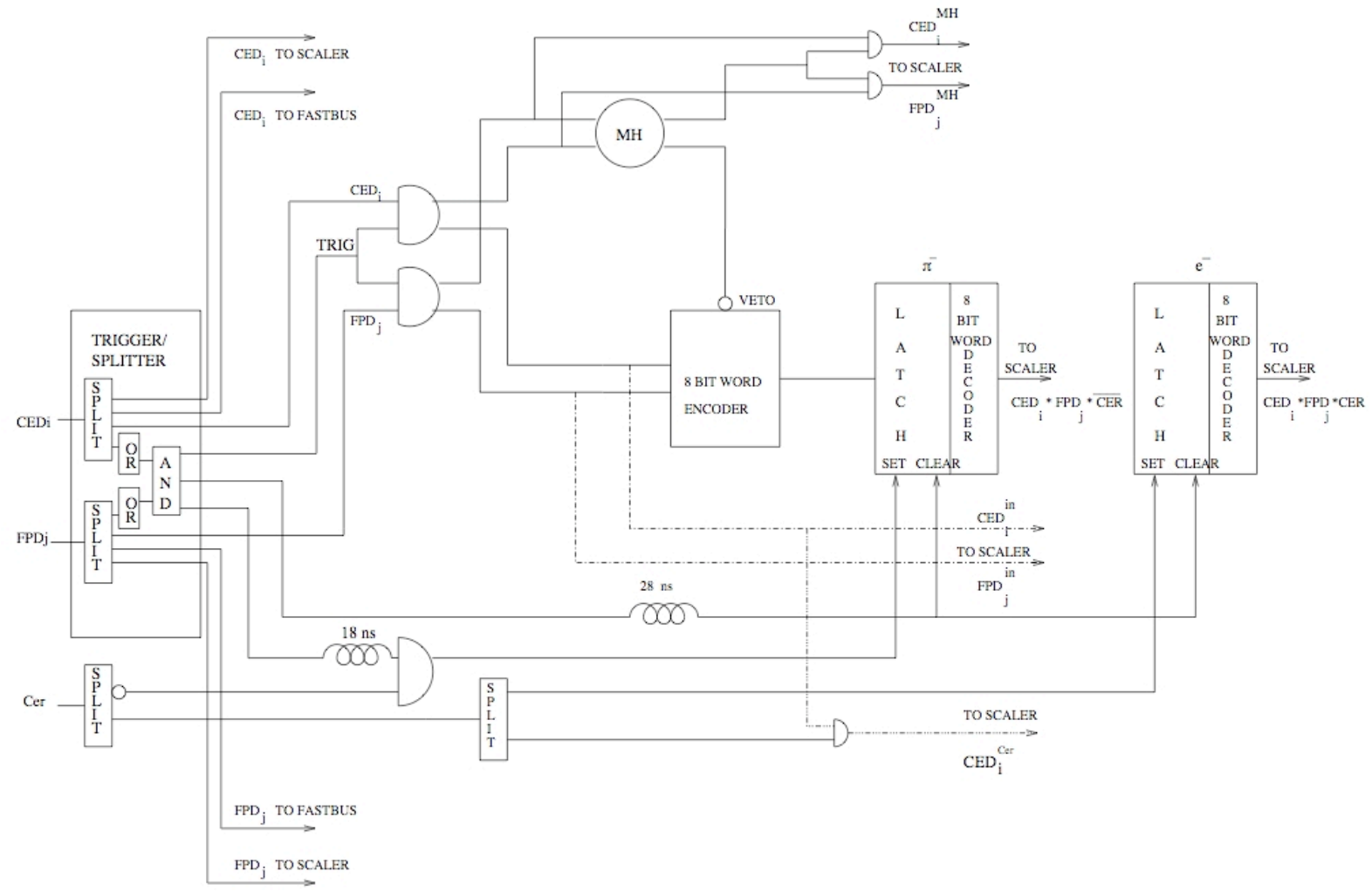

Figure 4.9: Schematic drawing of the NA electronics.

detectors, each photomultiplier tube had a signal which was also sent to a CFD. A trigger was generated whenever either two or three good CFD signals from different photomultiplier tubes within one Čerenkov detector were generated within a timing window. The number of required photomultiplier tubes was the "multiplicity", and the multiplicity varied depending on the experimental running conditions, but was usually set at two. When there was a "good" CED and FPD coincidence within the same timing window as a "good" Čerenkov trigger, the particle was identified as an electron. If there was a CED-FPD coincidence outside of the timing windows of proper Čerenkov triggers, the particle was classified as a pion. Each type of hit, whether it was a regular coincidence, a multi-hit coincidence, or single hits on the 


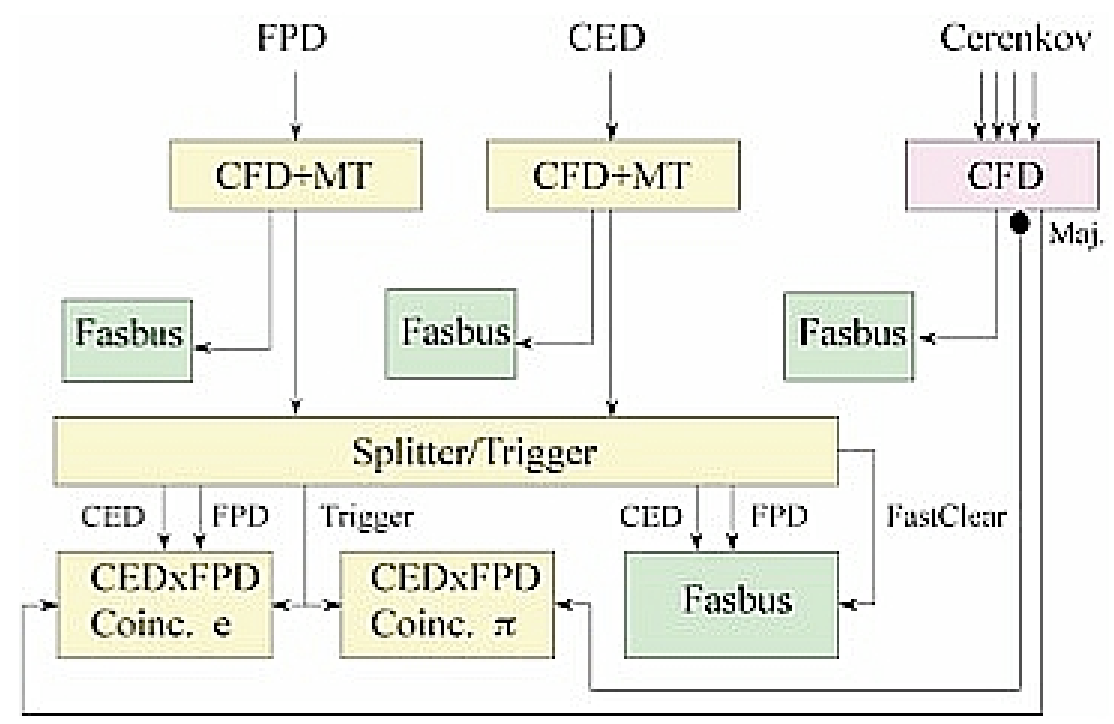

Figure 4.10: Schematic drawing of the French electronics.

CED, FPD, and the Cerenkov detectors, were sent to the scalers and recorded.

In addition to the primary acquisition electronics, conventional Fastbus ADCs and TDCs were used to acquire and store complete event-by-event data for a small fraction of beam pulses (less than $0.01 \%$ ) $\left[\mathrm{M}^{+} 08\right]$. These data allowed analysis and monitoring of the electronics. In addition, the Analog Ring Sampler (ARS), not shown in the electronics diagrams, was part of the monitoring electronics and consisted of a ring of 128 capacitors that continuously sampled photomultiplier tube signals at a rate of $1 \mathrm{GHz}$ and provided a means for limited time of flight analysis. Once an event was detected by the ARS trigger, the ARS made one more turn and then waited for a validating signal from the Fastbus trigger while the 128 cells were on hold. If a valid signal came within the proper timeframe, samples from all channels were converted by an ADC and stored digitally [Gou06]. The ARS could be used to determine how many photoelectrons were recorded per event per 
photomultiplier tube, if there were any correlations between photomultiplier tubes, and if the event should be considered a real electron or not. The ARS information could be used in many ways such as determining whether it was more efficient for the Čerenkov trigger to fire on at least one photoelectron on several photomultiplier tubes, or on several photoelectrons using the analog sum of all photomultiplier tubes, or to find the efficiency of the Čerenkov detectors. A detailed analysis of the Čerenkov detector efficiencies was also made using a special beam structure that allowed a time of flight analysis, [Ver08b, Ver08a].

\subsection{Data Acquisition}

The basic unit of the $G^{0}$ measurement was a macro-pulse (MPS) which was $1 / 30$ second in duration. The $G^{0}$ data acquisition system (DAQ) dealt with data having two different structures and sources. The primary data stream used a $30 \mathrm{~Hz}$ trigger and recorded information per MPS; the other data stream recorded Fastbus data and used a Fastbus trigger.

The $G^{0}$ DAQ was driven by the CEBAF Online Data Acquisition (CODA) system designed at Jefferson Lab. Data recording was initiated when the primary DAQ computer sent a trigger command to the Trigger-Supervisor (TS) which passed the signals to the readout controller (ROC) of each electronics crate. Data were accumulated for the duration of an MPS. At the end of each MPS, during the "helicity-stabilization" period, the data were stored in memory buffers to allow them to be read out during the next MPS. After each MPS, the beam parameters 
and event data were transferred to the appropriate ROC and then information from all of the ROCs was gathered together as a single event by the "Event Builder" in the DAQ computer $\left[\mathrm{M}^{+} 08\right]$. A set of slow control data was also recorded to monitor and control the experimental apparatus which included information such as detector high voltages, beam characteristics, target, and SMS parameters $\left[\mathrm{M}^{+} 08\right]$. Ethernet was used to interface the primary DAQ computer with each of the ROCs.

A schematic diagram of the $G^{0}$ Data Acquisition system is shown in Fig. 4.11. The data were stored in the standard CODA format where the data from a given ROC were written with a ROC number and the length of the data encoded in the band header [Liu06]. Data were collected in run periods that were approximately one hour in duration. At the completion of a run, the data were analyzed using a replay engine analysis software. Details of the replay engine will be presented in Sec. 5.1.

Real time monitoring of the data taking was possible because the CODA software wrote the data into an event-transfer buffer. These data could be read and analyzed using standard software packages that produced plots showing detector rates, beam qualities, etc. [Liu06].

\subsection{The $G^{0}$ Polarized Electron Beam}

The polarized electron beam used in the $G^{0}$ experiment was produced at the Jefferson Lab (JLab) Continuous Electron Beam Accelerator Facility (CEBAF), which is a five-pass recirculating accelerator capable of simultaneous delivery of con- 


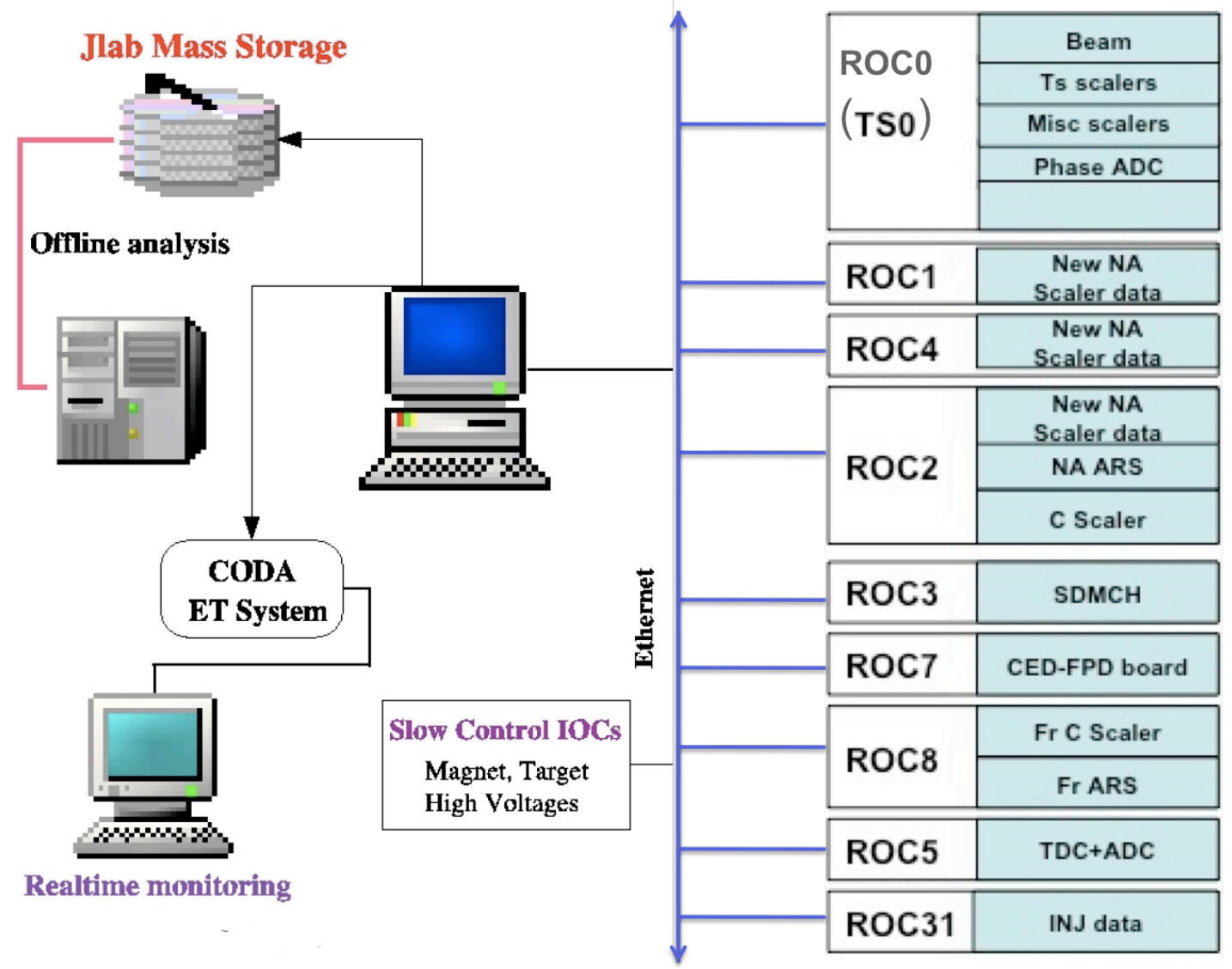

Figure 4.11: A block diagram of the $G^{0} D A Q$ system. ROCO contained the trigger supervisor (TSO), and the beam monitor scalers. ROC1 contained NA coincidence scalers. ROC 2 contained $10 \mathrm{NA}$ coincidence scalers, NA ARS and Čerenkov scalers. ROC3 contained eight French DMCH boards. ROC4 held $19 \mathrm{NA}$ coincidence scalers modules. ROC5 contained the Fastbus TDC and ADC modules. ROC7 contained the French CED-FPD coincidence boards, ROC8 contained the French Črenkov modules and the French ARS. ROC9 contained the NA singles scalers. ROC31 contained the beam injector data. Several modules not identified here are described in [Ben06]. 
tinuous beams to three end stations. CEBAF includes a polarized electron source, an injector where the beam is bunched prior to acceleration, two linear accelerators (linacs), two return arcs that move the beam from one linac to another for multiple acceleration, and the beam switch-yard where the beam is steered into one of three experimental halls. Hall $\mathrm{C}$ was used for the $G^{0}$ experiment. A schematic drawing of the JLab facility is shown in Fig. 4.12.

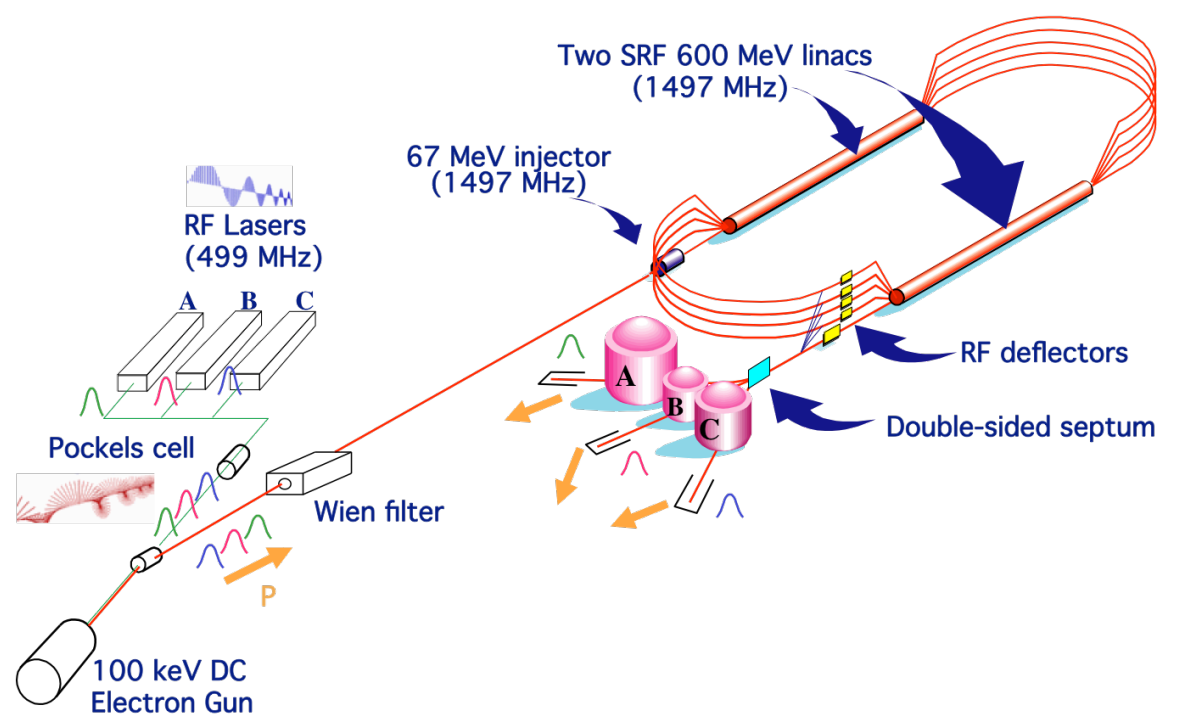

Figure 4.12: A schematic drawing of the JLab CEBAF accelerator [Gra06].

\subsubsection{CEBAF Accelerator}

The electron beam appears continuous, but in actuality it is comprised of pulsed beams, one for each experimental hall. The beams are pulsed at a very high rate, typically $499 \mathrm{MHz}$ with a $120^{\circ}$ offset for each beam, thereby forming a 3-beam, $1497 \mathrm{MHz}$ bunch train. After the bunch train is formed, the beams enter the injector portion of the accelerator. The injector simultaneously establishes the 
initial structure and energy of the three beams used in each of the experimental halls (see Fig. 4.13). Just after the beam enters the injector, the prebuncher anticipates and compensates for the lengthening of the bunch charge due to space charge forces as the beam travels between the cathode and the buncher. The chopper establishes the initial timing and proper longitudinal structure of each of the three beams. The capture section provides the initial acceleration of the beam to $500 \mathrm{keV}$. The bunch length cavity is part of a diagnostic system used to tune the beam's bunch length. This is followed by the first of two superconducting (SRF) cavities where the beams are further bunched and accelerated to $5 \mathrm{MeV}$. This is followed by eight SRF cavities which accelerate the beams to an energy of $23-68 \mathrm{MeV}$. At the end of the injector, the beam passes through a chicane before joining the re-circulated beams in the main accelerator $\left[\mathrm{R}^{+} 04\right]$. Once in the main machine, the beam is accelerated

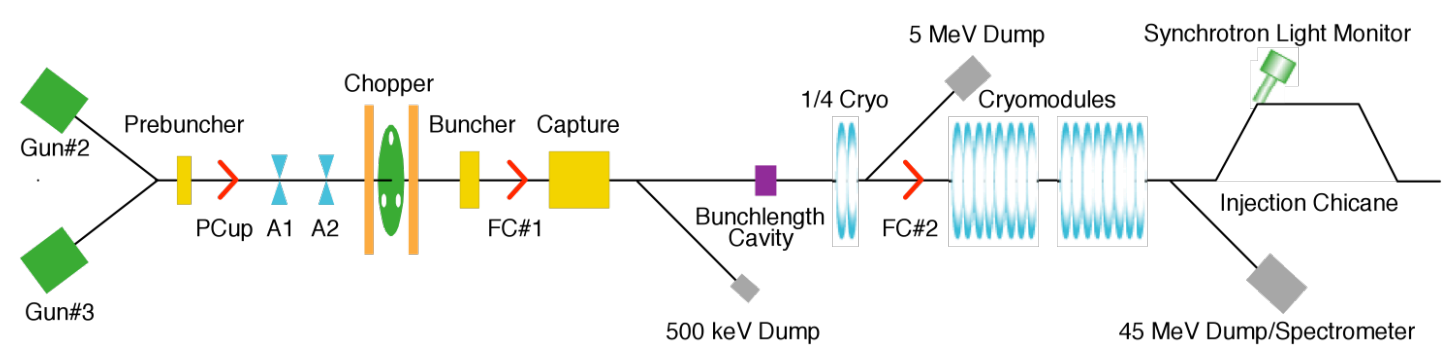

Figure 4.13: A schematic drawing of the JLab CEBAF injector [Gra06].

using a "racetrack" recirculating beam line. Twenty cryomodules, each containing eight superconducting niobium cavities line each of the two linacs. Each linac is set to the same energy and can provide up to $600 \mathrm{MeV}$ per pass. Liquid helium keeps the accelerating cavities superconducting at a temperature of 2 K. Quadrupole and dipole magnets in the tunnel focus and steer the beam as it passes through each 
arc. The three beams travel together in the linacs, but they are separated in the recirculation arcs because the strength of the bending fields required depends on the beam energy, and the energy of the three beams can vary significantly. In total, more than 2,200 magnets are necessary to keep the beam properly focused. An RF

separator is used to give the beam a transverse kick in order to steer it into one of the three halls or to recycle it back into the linacs for additional acceleration. The RF scheme uses $499 \mathrm{MHz}$ cavities, which kick every third bunch out of the machine. The accelerator can deliver the first four passes to one hall only. The fifth pass can be sent to all three halls simultaneously. [Acc09]

\subsubsection{Polarized Source}

Each hall illuminates a common photocathode using a polarized laser. During the $G^{0}$ backward angle measurement, Halls $\mathrm{A}$ and $\mathrm{B}$ shared a laser and Hall $\mathrm{C}$, where the $G^{0}$ measurement was made, had its own. The Hall C laser was a fiber laser installed in March 2006 for the backward angle running [Bai07]. The laser seed was a common cable TV communications laser diode which was fed into a commercial fiber laser amplifier which amplified the $1 \mathrm{~mW}$ seed to $5 \mathrm{~W}$ [HP06]. The $1560 \mathrm{~nm}$ amplified light was then sent through a second harmonic generator (SHG) where a portion of the light was shifted to $780 \mathrm{~nm}$-an ideal wavelength for the superlattice GaAs cathode. The fiber laser has many advantages over the Titanium-Saphire laser used during the forward angle experiment, including significantly higher power output, enhanced reliability, it never loses "lock", and the fiber laser has the ability 
to quickly shift the rf phase [HP06].

The linearly polarized light from the laser is converted to circularly polarized light by a helicity Pockels cell (HPC) which is an electro-optic device in which the birefringence is proportional to the applied electric field. The laser light is initially linearly polarized in the vertical direction. The optic axis of the HPC is aligned along the beam, with the birefringent axes at $\pm 45^{\circ}$ to the initial-state polarization. High voltage is applied to the HPC to create a $\pm \pi / 2$ phase shift between the birefringent axes thereby creating left or right-handed circular polarization[Pas07]. A simplified layout of the $G^{0}$ laser table is shown in Fig. 4.14.

The reversal frequency of the Pockels cell high voltage was chosen to be 30 $\mathrm{Hz}$ in order to cancel any potential $60 \mathrm{~Hz}$ noise due to the cycle of the power line [Liu06]. As mentioned previously, each $1 / 30 \mathrm{~s}$ window is a basic unit of measurement for the $G^{0}$ experiment and is called an MPS. Four MPS make up a "quartet" and it is the asymmetry of a detector yield for a quartet that is measured and recorded. A quartet is generated pseudorandomly as either +--+ or -++- , where "+" represents a positive helicity state (spin vector parallel to the beam) and "-" represents the negative helicity state, by using a software generated pseudorandom bit pattern to determine the first helicity state of each quartet [SFD05]. Between MPSs there is a period of $200 \mu s$ where the Pockels cell has time to stabilize [Bai07].

The circularly polarized laser light is directed onto a photocathode where electrons are produced via photoemission. Photoemission of electrons from a semiconductor requires excitation of the electrons into the conduction band, transport of electrons to the semiconductor's surface, and then emission into the vacuum. GaAs 


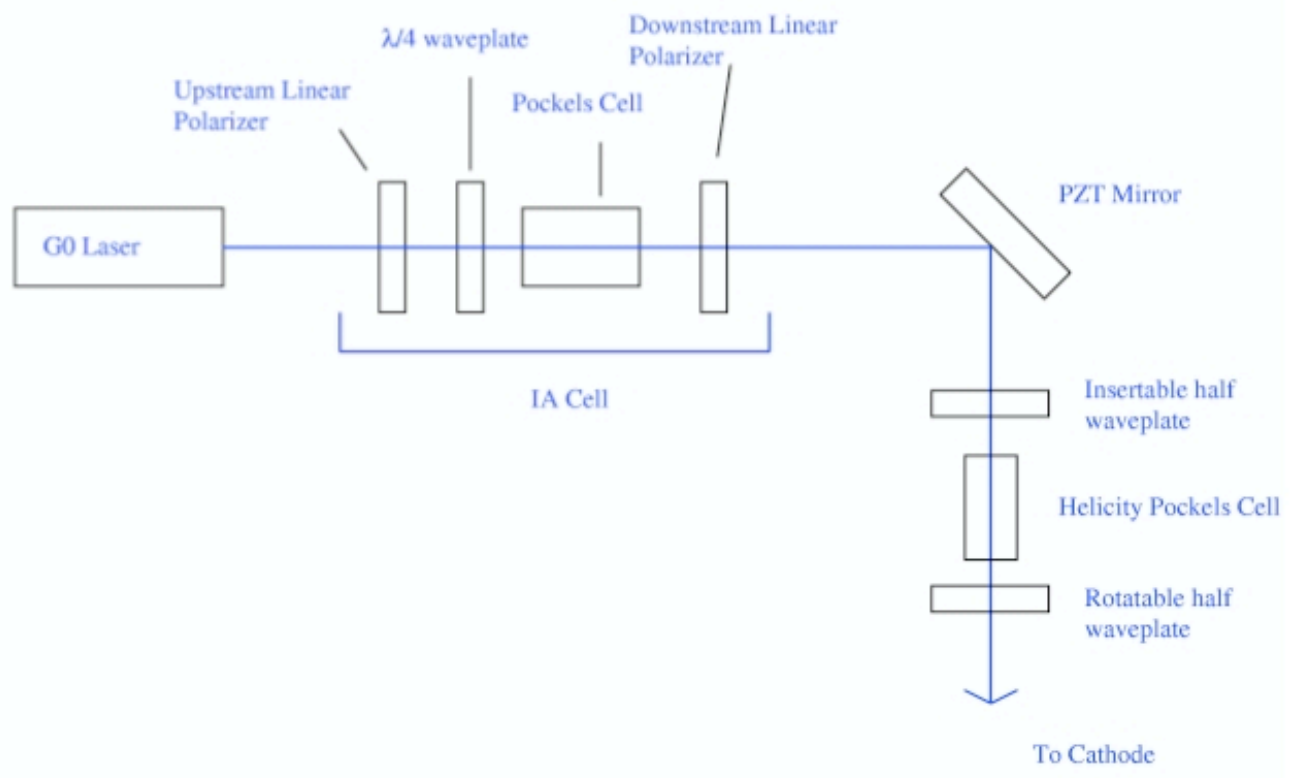

Figure 4.14: A simplified schematic drawing of the $G^{0}$ laser table.

has the very favorable property that the vacuum level can be lowered below the bulk conduction band minimum by application of cesium and oxygen. This condition is called negative electron affinity (NEA) and the result is that the depth from which electrons can be emitted is not limited by the hot electron mean free path $(\approx 10 \AA)$, but rather by the diffusion length $(\approx 1 \mu \mathrm{m})$ for electrons thermalized to the conduction band minimum $\left[\mathrm{P}^{+} 80\right]$. Conduction band electrons can escape from the surface even if the photon interaction takes place deep within the material. NEA GaAs is an extremely efficient photoemitter and enjoys widespread use in photomultiplier tubes, night vision devices, etc. Fortunately, one of the best photoemitters is also an efficient source of spin-polarized electrons. An important characteristic of a GaAs source is that the sign of the spin polarization of the excited electrons can be easily changed by reversing the helicity of the incident light without affecting other pa- 
rameters of the electron beam [PC01]. A Superlattice GaAs photocathode was used during the $G^{0}$ backward angle measurement and achieved a polarization in excess of $85 \%$. The forward angle measurement used a strained GaAs photocathode and had an average polarization of $74 \%$. A discussion of strained and superlattice GaAs photocathodes can be found in $\left[\mathrm{B}^{+} 05\right]$.

An insertable half-wave plate (IHWP) was used to check for systematics by flipping the handedness of the circular polarization and therefore the helicity of the beam. It was located on the injector laser table and its insertion or retraction remotely controlled. When the IHWP state was changed, the real physics asymmetry changed sign, but any false asymmetries due to systematics would not. If the sum of the asymmetries from the two IHWP states does not equal zero, then false asymmetries due to the $G^{0}$ electronics are present [Gra06].

\subsubsection{Beam Monitors}

The parity-violating asymmetry in e-p and e-d scattering is sensitive to helicitycorrelated fluctuations which may arise from a number of sources. One possibility is an anisotropy in the GaAs crystal that results in asymmetric electron yields. This would induce a helicity-correlated intensity asymmetry $\left[\mathrm{C}^{+} 04\right]$. Pockels cell lensing may steer the laser beam off-center thereby inducing a helicity-correlated position difference. If the Pockels cell has an intrinsic birefringence gradient, the spatial variation of the laser polarization may shift the beam centroid inducing a helicity-correlated position difference [Gra06]. These fluctuations must not only be 
minimized, but they need to be accurately monitored and measured so that any false asymmetry induced by these fluctuations can be calculated and subtracted from the measured asymmetry.

Beam Current Monitors (BCMs) include an Unser monitor and two resonant cavities positioned one on either side of the Unser. The Unser is a toroidal transformer designed as an absolute monitor providing a direct measurement of the intensity of the beam current. The Unser is very accurate at high currents but because of its unstable offset, it is unreliable during routine operations. The cavities are "relative" current monitors that are stable and linear over a large dynamic range. They are calibrated with the Unser at high currents and then used instead of the Unser during normal run periods [Bai07].

Vertical and horizontal beam position is measured using Beam Position Monitors (BPMs) which are a 4-wire array of open-ended wire strip lines tuned to the fundamental accelerator frequency $(1497 \mathrm{MHz})$. The strip lines run along the inside length of a cylinder, as shown in Fig. 4.15. When the beam passes through the cylinder, a current is induced in each strip line; if the beam is closer to one strip line than another, then the relative magnitude of the signals determine the position of the center of mass of the beam [Bai07].

The beam "Halo" consists of electrons that have strayed more than $2 \mathrm{~mm}$ from the central core of the beam. Halo can be generated from beam scrapes against the beam pipe, self-interaction of the beam, and an improperly tuned beam in the injector. Halo was not a concern from a helicity-induced false asymmetry perspective, but if large enough, halo could damage photomultiplier tubes by dramatically 


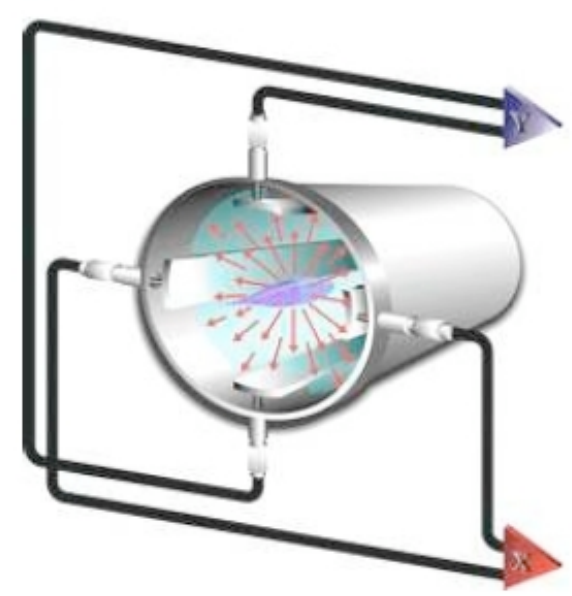

Figure 4.15: A diagram of a BPM, showing the four strip lines running along the inside of the cylinder [BaiOr].

increasing the singles rate seen in the detectors. Halo can contribute significantly to background events including those generated from scattering from target walls, and was monitored aggressively to keep it at a minimum. Halo was measured using a target that could easily be moved in and out of the beam line, and was simply a hole in a sheet of carbon that the beam passed through. There were three halo targets with radii of $5.5 \mathrm{~mm}, 3 \mathrm{~mm}$, and $2 \mathrm{~mm}$. The $2 \mathrm{~mm}$ target represented the nominal halo limit for the experiment. The larger targets provided information on the extent of the halo. If halo was present, then scraping of the beam along the halo target edges created a shower of particles that were then detected in the halo monitors located immediately downstream of the halo target. For specifics on the halo monitors including locations along the beam line, see [Bai07].

The other beam monitors were the eight luminosity monitors placed at very forward angles on the beam line to measure target density fluctuations as well as beam induced false asymmetries. A complete description of the luminosity monitors 
can be found in Appendix B.

\subsubsection{Polarimetry}

The incomplete beam polarization was a dilution factor to the parity-violating asymmetry measured during the $G^{0}$ experiment and therefore had to be accurately measured. For the backward angle measurement, two polarimeters were used, one was a Møller polarimeter and the other was a Mott polarimeter, both are described below.

The Møller polarimeter measured the spin-dependent cross section asymmetry $A_{M \varnothing l}$ for elastic scattering of polarized electrons from polarized electrons. The cross section asymmetry for elastic $(\vec{e}-\vec{e})$, or Møller, scattering can be calculated to high precision using quantum electrodynamics. When both the beam and target electrons are polarized, the cross section, $\sigma$, in the center of mass frame can be expressed as [Lop96]:

$$
\frac{d \sigma}{d \Omega}=\frac{d \sigma_{u n p o l}}{d \Omega}\left[1+P_{t} P_{b} A_{z z}(\theta)\right]
$$

where $P_{b}$ and $P_{t}$ are the longitudinal polarization of the beam and the target electrons respectively, $A_{z z}$ is the analyzing power, $\theta$ is the center of mass scattering angle, and $\frac{d \sigma_{\text {unpol }}}{d \Omega}$ is the unpolarized cross section. The asymmetry can then be expressed as [Lop96]:

$$
A_{M \varnothing l}=\frac{\frac{d \sigma \uparrow \uparrow}{d \Omega}-\frac{d \sigma \uparrow \downarrow}{d \Omega}}{\frac{d \sigma \uparrow \uparrow}{d \Omega}+\frac{d \sigma \uparrow \downarrow}{d \Omega}}=P_{t} P_{b} A_{z z}(\theta)
$$

with

$$
A_{z z}=-\sin ^{2} \theta \frac{8-\sin ^{2} \theta}{\left(4-\sin ^{2} \theta\right)}
$$


When $\theta=90^{\circ}$, the analyzing power is at a maximum and $A_{z z}=-7 / 9$ [Lop96]. Therefore, if the target polarization is well-known, and the kinematics can be arranged so that $\theta=90^{\circ}$, the beam polarization can be measured by comparing the cross section asymmetry for beam and target spins aligned parallel to one another and anti-parallel.

The Hall $\mathrm{C}$ polarimeter is located in the beam alcove, which is upstream of the entrance to Hall $\mathrm{C}$, but downstream of the last dipole steering magnets for the beam. The layout of the Møller polarimeter is shown in Fig. 4.16. The Møller

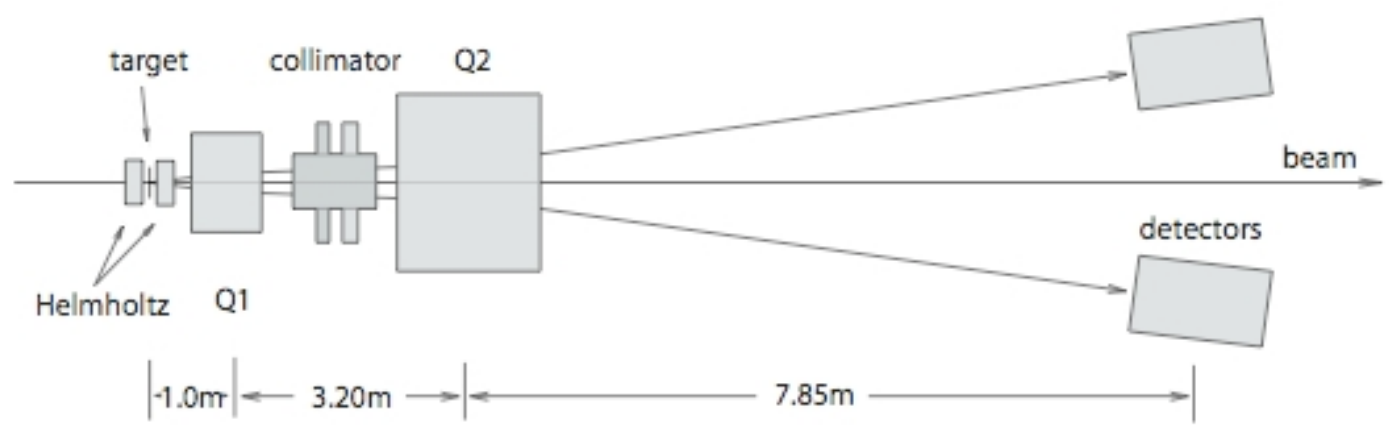

Figure 4.16: The layout of the Hall C Møller polarimeter

target was typically a thin foil of pure iron because the polarization of iron is known to great accuracy in saturation: $P_{t}=8.04 \% \pm 0.02 \%$ [Lop96]. The typical target used for the $G^{0}$ measurements was a strip of iron foil $4 \mu \mathrm{m}$ thick that was placed perpendicular to the incoming beam, between two Helmholtz coils that produced a $4 \mathrm{~T}$ field and saturated polarization in the iron.

Møller electrons that scatter at $\theta_{C M}=90^{\circ}$ passed through a small quadrupole, Q1, a series of tungsten-alloy collimators, and then a large quadrupole magnet, Q2, in order to ensure satisfactory separation of the scattered Møller electrons and the 
beam line [PGSH05].

The Møller polarimeter was designed for measurements at momenta above $800 \mathrm{MeV}$. By shifting the first quadrupole approximately 8.5 inches closer to the superconducting solenoid, Møller measurements were able to be made at $687 \mathrm{MeV}$ [GH08]. It was not possible however, to make Møller measurements at $362 \mathrm{MeV}$. The Mott polarimeter installed in the $5 \mathrm{MeV}$ region of the injector was used to make polarization measurements during the low energy backward angle runs and provided a consistency check with the Møller measurements during the $687 \mathrm{MeV}$ runs [GH08].

The principle of Mott polarimeters is that polarized electrons are scattered by the Coulomb field of a heavy, unpolarized nucleus, where the scattering probability depends upon the polarization of the electron that is scattered. Mott polarimeters measure the left-right asymmetry in the scattering of polarized electrons from atoms. The largest asymmetries are for electrons scattered at large angles from high-charge nuclei, which is why gold foil is a common Mott target [Sin98].

The Mott polarimeter, located in the injector region of the accelerator, is shown in Fig. 4.17. During a polarization measurement, a $12.5^{\circ}$ dipole bend magnet was energized to guide the electron beam to the Mott target. The target was mounted on a moving ladder that allowed selection of 17 different targets [Bai07]. Maximum analyzing power was at $72.5^{\circ}$ and two sets of detectors were placed at this location to measure the transverse components of the electron's polarization[Sin98]. An adjustable aluminum collimator inside the vacuum chamber defines the acceptance of each detector. 


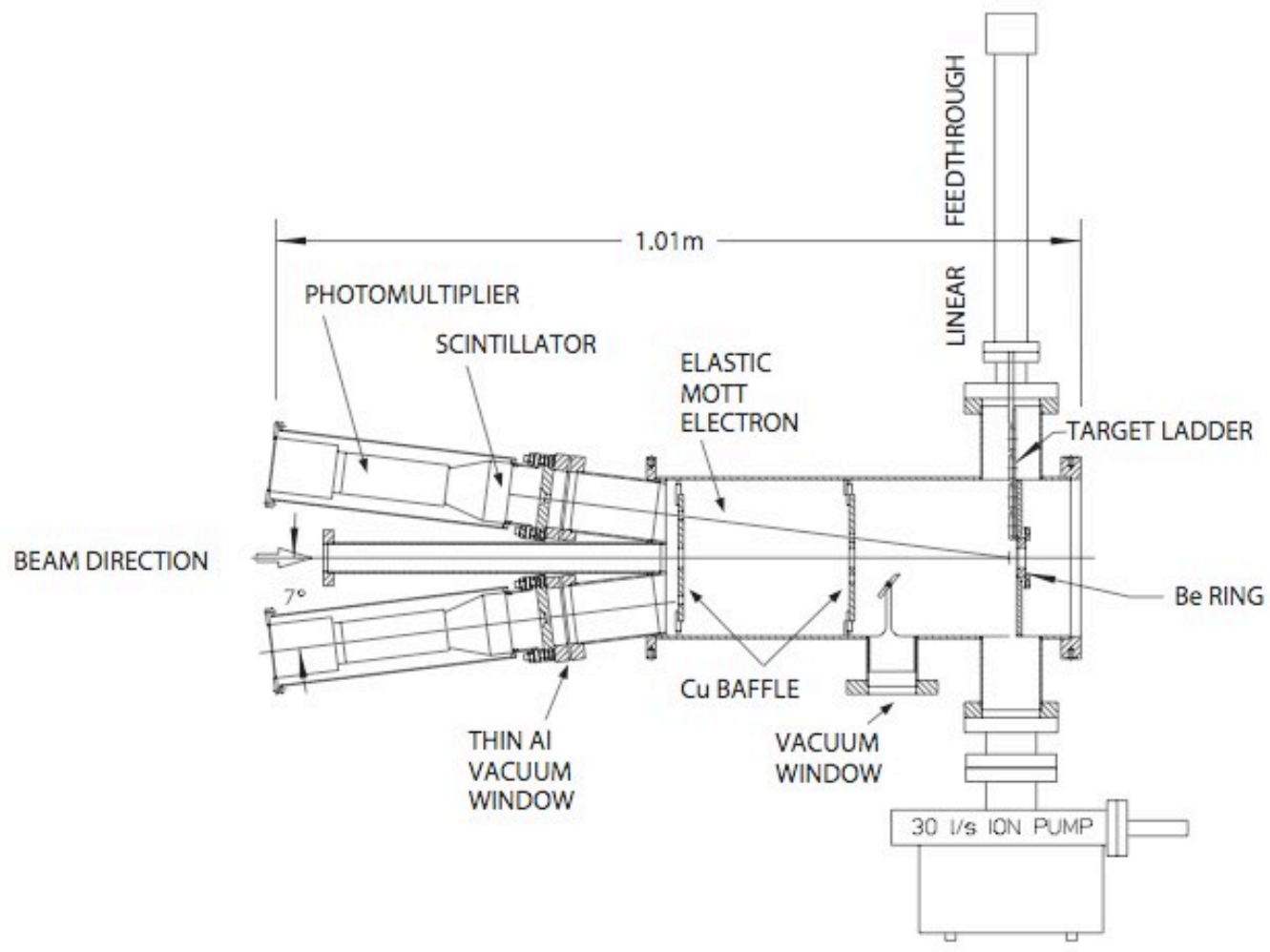

Figure 4.17: The layout of the Hall C Mott polarimeter. The beam direction is from left to right. 


\subsection{Target}

This section describes the main features of the $G^{0}$ target. See [Co05] for a complete description of the target system. The backangle measurement used an unpolarized cryogenic target specifically designed for the $G^{0}$ experiment. A relatively long target was needed in order to ensure high luminosity and achieve good statistical precision. It was designed to fit inside a cylindrical volume approximately $61 \mathrm{~cm}$ in diameter located within the vacuum enclosure of the $G^{0}$ superconducting magnet system. Additionally, it had to accommodate large power depositions without inducing large systematic uncertainties caused by either global yield reductions or target density fluctuations on the time scale of $30 \mathrm{~Hz}$. To accommodate these constraints, a recirculating closed loop high power cryogenic target, shown in Fig. 4.18, was placed within the liquid nitrogen shield of the superconducting magnet, in the same plane as the beam line [Co05].

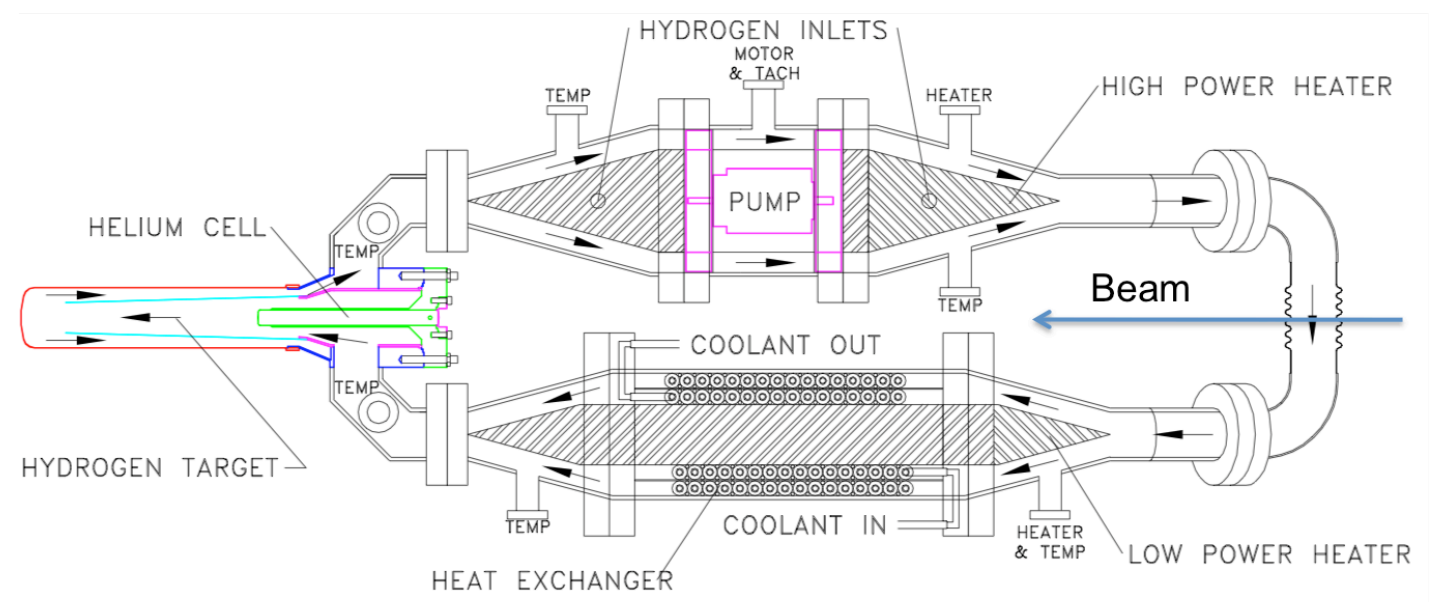

Figure 4.18: A schematic drawing of the $G^{0}$ target loop.

The cryogenic loop consisted of a heat exchanger, a recirculating pump, and 
the target manifold. With the exception of the cryogenic motor rotor, all materials used in the fabrication of the target cryogenic loop were either low magnetic susceptibility or non-magnetic materials. The rotor of the cryogenic motor is made of strong permanent rare-earth magnets [Co05].

The target's service module is shown in Fig. 4.19. Its primary function was to support the cryogenic loop and to provide motion control in the $x$ and $y$ directions to allow centering of the target in the beam and movement of the target out of the beam (it is stationary in $z$, which is parallel to the beam line.) The service module also provided the interface for the gas and electrical lines between the target and the experimental hall environment.

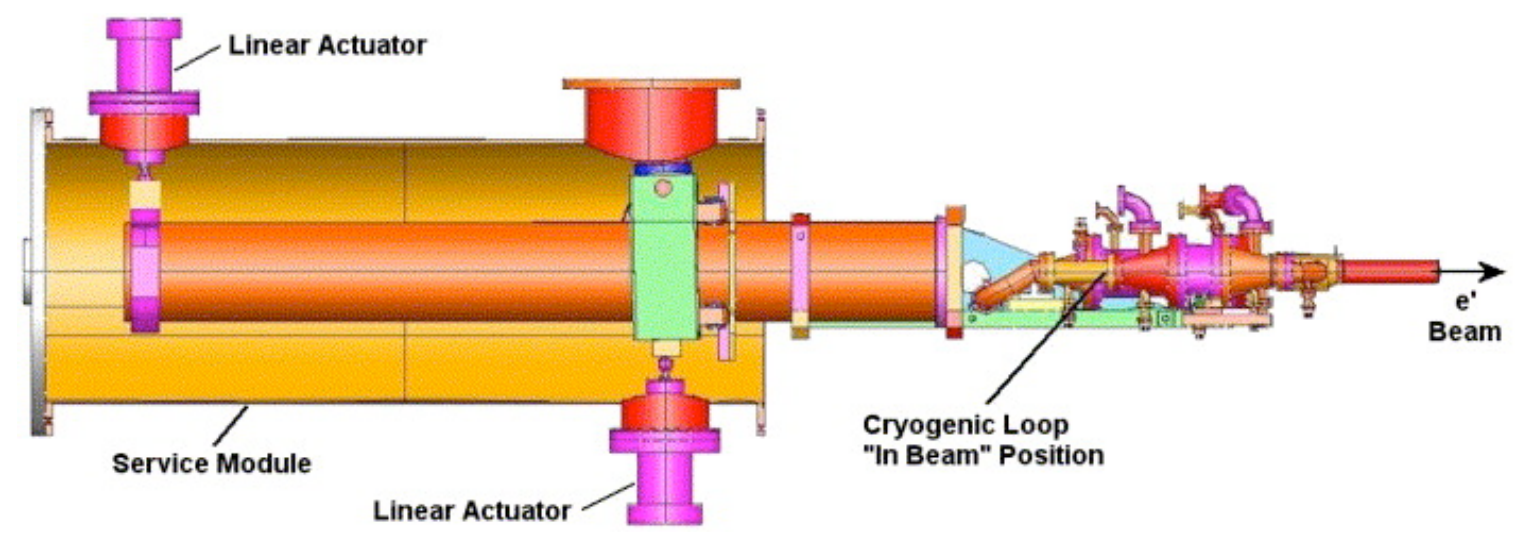

Figure 4.19: The target service module with the target in the "in beam" position. [Co05]

One of the legs of the cryogenic loop, housed a heat exchanger (see Fig. 4.18) which used compressed helium gas $(15 \mathrm{~K}, 12 \mathrm{~atm})$ as a target coolant. It removed approximately $50 \mathrm{~W}$ per g/s of coolant flow [Co05]. The other leg of the cryogenic loop housed the cryogenic pump and the high-power heater (HPH). The pump was a vane-axial design with two impellers in series. There were flow diverters 
inside the cryogenic loop, designed to guide the fluid smoothly around the loop and trigger a turbulent flow in the target cell and heat exchanger. Turbulence facilitates heat transfer and mixing. The HPH regulated the heat load on the target, with its primary function being to compensate for large reductions in beam current. Temperature sensors were located at various locations in the loop and a Proportional Integrated Differential (PID) feedback system was used to maintain a constant loop temperature. The system monitored the beam current and regulated the output of the HPH to maintain a nearly constant total combined power from both the beam and the HPH. This feedback system ensured that even when the target went from receiving $60 \mu \mathrm{A}$ of current at $687 \mathrm{MeV}$ to beam off, the temperature of the target varied less than $0.2 \mathrm{~K}$.

The target manifold was constructed of aluminum, while the shell of the rest of the loop was made of Type 304 stainless steel. The manifold, as shown in Fig. 4.20, housed two cells, a hydrogen target cell and a helium cell. The hydrogen cell was the primary target cell and could be filled with either hydrogen or deuterium. Its inner diameter measured $5 \mathrm{~cm}$, its length was $20 \mathrm{~cm}$, and it had a shell thickness of $0.178 \mathrm{~mm}$. The downstream wall of the cell served as the exit window for the beam. The center of this wall was machined to a thickness of $0.0762 \mathrm{~mm}$ within a radius of $4 \mathrm{~mm}$ from the center; the remainder of the exit window was the same thickness as the rest of the hydrogen shell, $0.178 \mathrm{~mm}$. The helium cell was $16 \mathrm{~cm}$ long with an inner diameter of $12.7 \mathrm{~mm}$.

The upstream portion of the loop was situated below the beam line, Fig. 4.19. Therefore, the beam passed through three aluminum windows on its way to and 


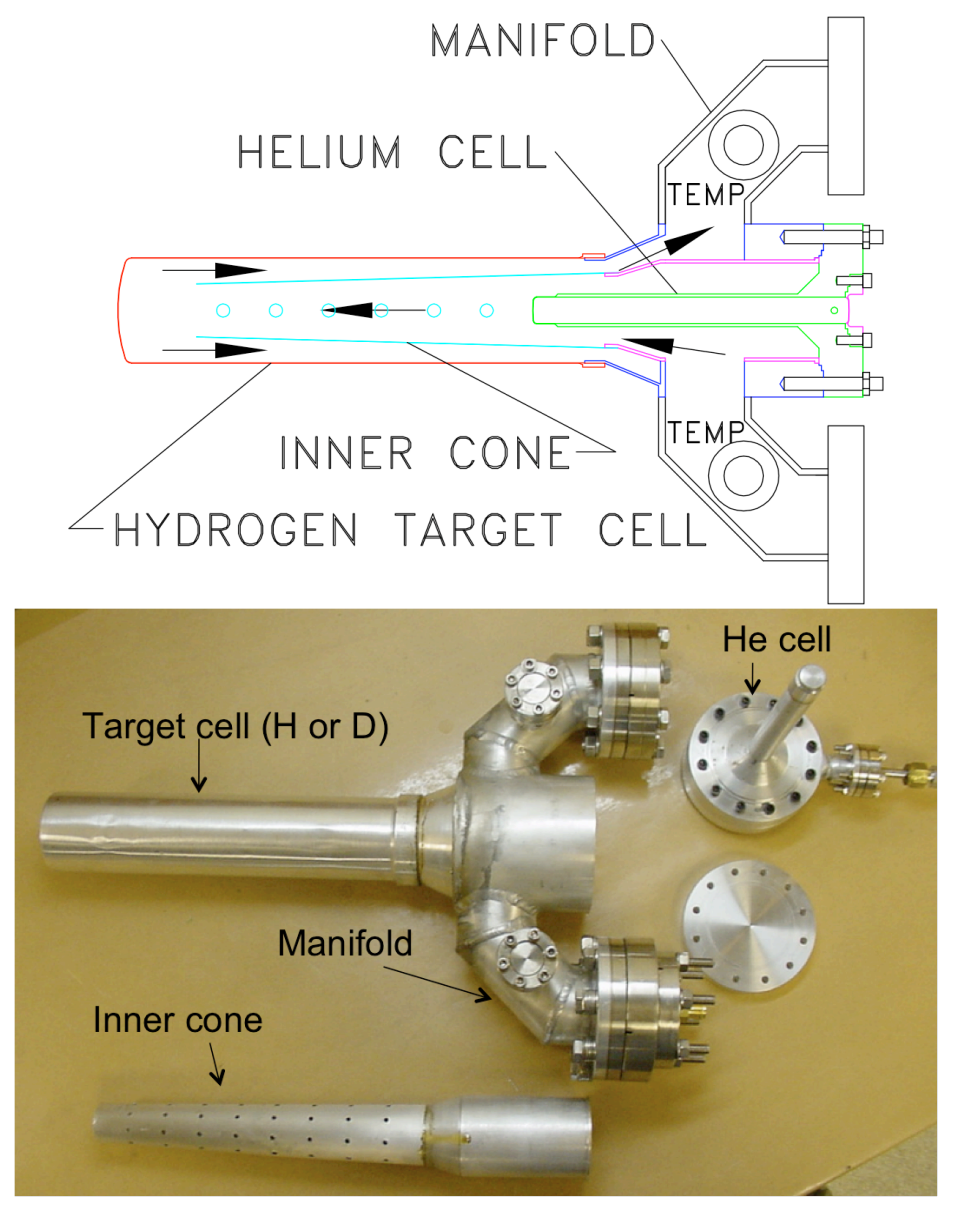

Figure 4.20: A schematic drawing [Co05] and photograph of the $G^{0}$ target cell and manifold. 
through the target: the helium cell entrance window, the helium cell exit window (which was also the hydrogen cell entrance window) and the hydrogen cell exit window. These thicknesses were $0.228 \mathrm{~mm}, 0.178 \mathrm{~mm}$, and $0.0762 \mathrm{~mm}$ respectively. The distance between the exit window of the helium cell and the exit window of the hydrogen cell was $20 \mathrm{~cm}$ and defined the liquid target in the beam. The beam was rastered in a uniform square pattern on the target with a nominal size of $1.8 \mathrm{~mm} \times$ $1.8 \mathrm{~mm}$.

The helium cell was maintained at the same pressure and temperature as the hydrogen cell which was nominally 1.7 atm with temperatures of $19 \mathrm{~K}$ for liquid hydrogen and $22 \mathrm{~K}$ for deuterium. The pressurized helium cell had a spherical convex target entrance window that matched the hydrogen cell exit window with a radius of curvature of $7.6 \mathrm{~cm}$. This eliminated any first-order variations in target length with beam position by matching the entrance and exit window curvatures. The other advantage of having the helium gas cell was that the asymmetric joints of the manifold with the cryogenic loop were moved further upstream and therefore outside of the detector acceptance. Also within the hydrogen cell was an inner cone which served as a flow diverter and guided the target liquid down the center of the target. The conical geometry increased the speed of the fluid flow thereby increasing the turbulence and mixing of the fluid. Additionally, the inner cone had holes that allowed faster heat removal from the fluid along the beam path as well as help to relieve static pressure along the inside of the inner cone.

The target loop could also be filled and used with gaseous hydrogen to allow studies of the background contribution arising from the target structure. In addition 
to the cryotarget, an actuator system allowed selection of other target "types" including carbon and aluminum. These solid targets were used to measure background contributions due to the aluminum windows and to determine the effects of density fluctuations, or target "boiling" by comparing solid and liquid target results. The solid targets were located $1.7 \mathrm{~cm}$ upstream of the entrance window. The aluminum target had a thickness of $0.307 \mathrm{~cm}$.

Global density fluctuations of the target and target "boiling" were of concern and were carefully measured and monitored. Initial testing was reported in [Co05], where the operating conditions were $40 \mu \mathrm{A}$ beam current, a $2 \mathrm{~mm} \times 2 \mathrm{~mm}$ rastered beam size and $31 \mathrm{~Hz}$ pump speed. These tests showed a global density reduction $<1.5 \%$ as compared to no beam on target. The density fluctuation contributed 238 $\pm 65 \mathrm{ppm}$ to the width of the measured asymmetry distribution, where a typical detector asymmetry width was 1200 ppm [Co05]. These tests and measurements were made using a special set of luminosity detectors that were located at very forward beam angles. For the backward angle measurement, typical asymmetry widths attributed to target density fluctuations were on the order of $290 \mathrm{ppm}$ for $60 \mu \mathrm{A}$ of beam current on a hydrogen target and approximately $110 \mathrm{ppm}$ for $25 \mu \mathrm{A}$ of beam current on a deuterium target. A complete description of the luminosity detectors and density fluctuation testing for the backward angle experiment can be found in Appendix B. 


\section{Chapter 5}

\section{Data Analysis}

This section details how the data went from "raw" asymmetries measured one quartet at a time, to a final physics asymmetry for each data set. The analysis software used to process the raw event data is discussed as well as the procedures to correct the data for dilutions, random events, equipment dead time, helicitycorrelated false asymmetries, radiative corrections, and beam polarization.

\subsection{Data Reduction Procedure}

The data were collected and stored by the DAQ (see section 4.8) in roughly one hour time periods to segment the raw data into runs. Each run was assigned a six-digit run number. The unprocessed data were analyzed using the $G^{0}$ replay engine. An overview of the replay engine is presented here, and a more thorough discussion can be found in section 5.1.1. The replay engine was analysis software written in $\mathrm{C}++$ and $\mathrm{ROOT}[\mathrm{ROO}]$ that extracted physics quantities and organized the data for each run in the form of ntuples, both by MPS and by quartet. ROOT is an object-oriented analysis framework developed at CERN. At the end of a replay the data were in the form of a much reduced data set, which was recorded by run number in the $G^{0}$ database and, if desired, could also be written as a rootfile.

The $G^{0}$ databases were written in MySQL (Structured Query Language) [MyS]; 
the basic usage of the databases is describe below, but a more detailed discussion of their structure and mechanics can be found in Appendix $\mathrm{C}$ of this document.

At the beginning of a replay, instrument calibrations such as offsets and gains, were read from the database. At the end of a replay, two kinds of information were written to the database. The first was information describing the run conditions which included target type, target loop pressures and temperatures, SMS current, and several other measures of the real-time running conditions. The other represented the primary analysis result, comprised mainly of calculated values for the average detector yields and asymmetries including beam charge, position, halo, and luminosity monitors, as well as the total number of MPS and quartets for each run. Detector yield and beam monitoring data were calculated MPS by MPS while all asymmetries and helicity-correlated differences were calculated quartet by quartet. Also calculated were the linear regression slopes (discussed in Section 5.2.3).

Each run was analyzed four times using the $G^{0}$ replay engine, where each analysis replay was called a "pass", and each pass was defined by which types of corrections were applied to the data. This was done because the order in which the corrections were applied to the asymmetries and yields was important, and a correction often relied on a previous yield and asymmetry calculation made by the replay engine in order to apply the next correction. Although each analysis pass will be described in more detail later, a summary of the passes are as follows:

- Pass 1 - No corrections to the data except that any data failing to pass cuts that identified good events were ignored 
- Pass 2 - Cut applied to the quartet yield at $5 \sigma$ from the average mean yield distribution to correct a scaler bit error

- Pass 3 - Rate corrections due to the electronics - dead time, accidental coincidences

- Pass 4 - Linear Regression applied

All raw asymmetry values were "blinded" in order to avoid biasing the final measured physics asymmetry. This was accomplished by using an algorithm to multiply the raw asymmetries by a factor that fell within the range of $0.75-1.25$ just prior to writing the values to the database, see Table 5.1. There was a different blinding factor for each target and energy combination. This multiplicative factor was not revealed to anyone nor removed from the data until all corrections to the asymmetry were completed.

Table 5.1: Blinding factors applied to the raw asymmetries.

\begin{tabular}{||c|c|c||}
\hline \hline Beam Energy $(\mathrm{MeV})$ & Target & Blinding Factor \\
\hline \hline 687 & $\mathrm{H}$ & 1.23932 \\
687 & $\mathrm{D}$ & 1.12257 \\
362 & $\mathrm{H}$ & 1.09657 \\
362 & $\mathrm{D}$ & 1.01295 \\
\hline \hline
\end{tabular}

\subsection{1 $G^{0}$ Replay Engine}

Fig. 5.1.2 shows the data flow for the $G^{0}$ replay engine. After the detector and monitor calibrations were read from the database, each event was examined 
to determine whether it could be classified as a good event, by passing a series of cuts that were imposed MPS by MPS to ensure data quality. If the beam current varied by more than $3 \mu \mathrm{A}$ between MPS in any given quartet, those MPS were cut. Following a beam trip, there is a finite amount of time required to stabilize the beam and the target. To avoid including events prior to the beam being stable, the first 500 MPS ( $\approx 17$ seconds) following a beam trip are ignored. Any data collected with a beam current below $0.6 \mu \mathrm{A}$ were also discarded.

If all of the cuts were satisfied, the replay engine then checked to see which corrections needed to be calculated. These corrections included the scaler counting rate error correction (pass2), corrections for the electronics (pass3), and corrections for helicity-correlated beam parameters (pass 4). Each is explained in the sections that follow. Once the appropriate corrections were applied, the quartets were formed and the asymmetry and yield were calculated for a quartet. A quartet is comprised of four consecutive MPS that have all passed the necessary cuts, and as stated previously, is of the form +--+ or -++- where $+(-)$ represents the positive (negative) helicity state. Therefore, the asymmetry can be written as:

$$
A_{q r t}=H \frac{Y_{1}+Y_{4}-\left(Y_{2}+Y_{3}\right)}{Y_{1}+Y_{4}+Y_{2}+Y_{3}}
$$

where $H=1(-1)$ represents the positive (negative) helicity state and $Y_{i}$ represents the yield measured in the $i$ th MPS.

Helicity-correlated differences for beam positions (i.e. $\Delta x$ or $\Delta y)$, angles $\left(\Delta \theta_{x}\right.$ or $\left.\Delta \theta_{y}\right)$, and energy $(\Delta E)$ were calculated as:

$$
\Delta p=H \frac{p_{1}+p_{4}-\left(p_{2}+p_{3}\right)}{2}
$$




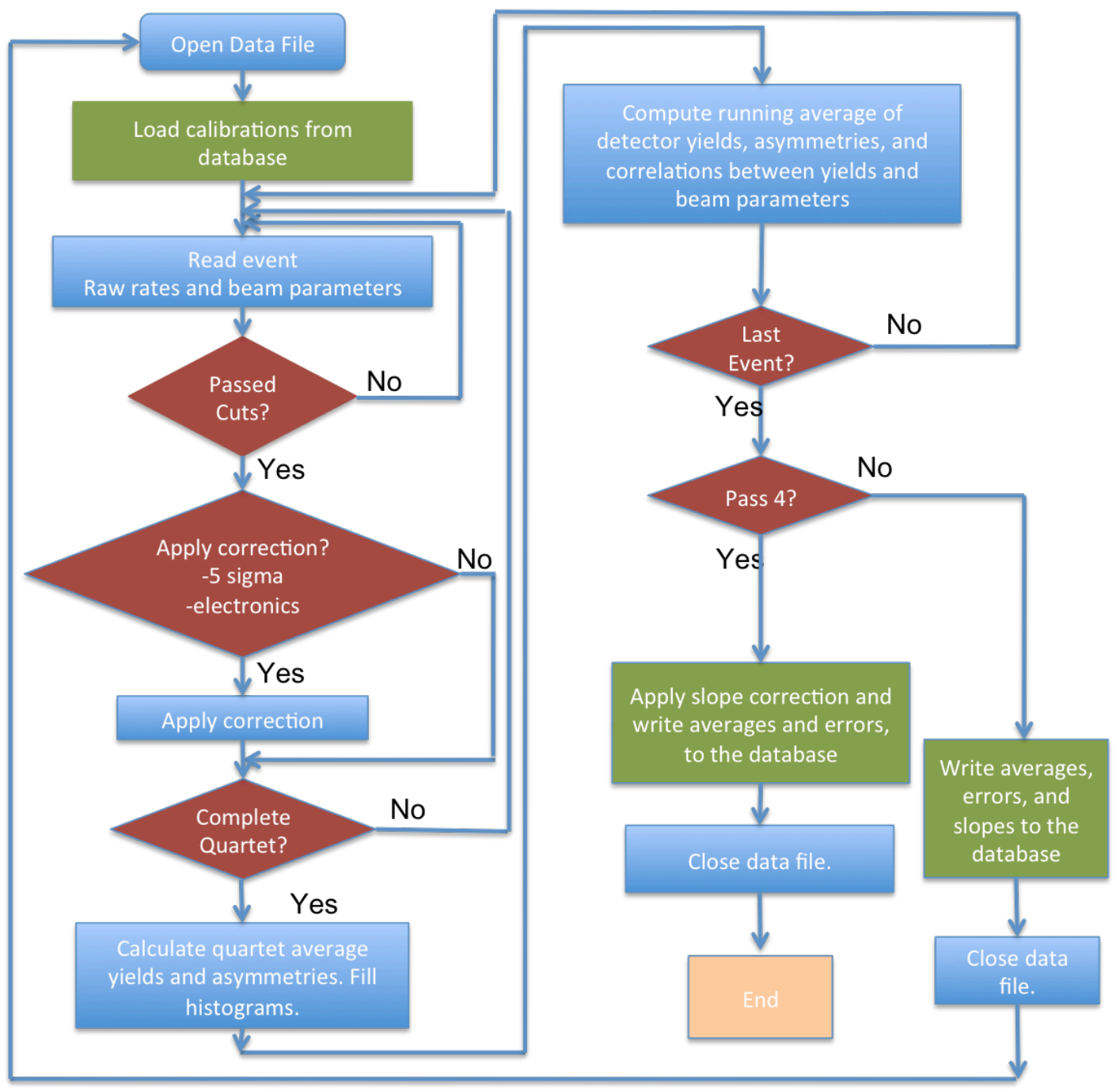

Figure 5.1: A diagram of the data flow for the $G^{0}$ replay engine. 
where $p_{i}$ represents one of these beam quantities for the $i$ th MPS. The straight average for each of these quantities, or in other words, the helicity-uncorrelated average was also calculated. Detector yields, detector asymmetries, helicity-correlated, and helicity-uncorrelated beam parameters were calculated for each valid quartet. Also calculated by quartet for each replay of each run, were the total running averages of these quantities and the square of these quantities, $\langle q\rangle$ and $\left\langle q^{2}\right\rangle$, to ease the calculation of the uncertainty of the mean for quantity " $q$ ", which can be expressed as

$$
\sigma(q)=\frac{\left\langle q^{2}\right\rangle-\langle q\rangle^{2}}{N_{q r t}}
$$

where a normal distribution is assumed and $N_{\text {qrt }}$ is the total number of good quartets for the run. The other quantities that were calculated and stored for each run, were the correlation slopes between detector yields and a beam parameter, $\frac{\partial Y}{\partial p}$. A more detailed discussion of the correlations slopes and the linear regression correction can be found in Sec. 5.2.3.

\subsection{2 $G^{0}$ Backward Angle Data}

As mentioned in section 4.7, the $G^{0}$ electronics identified valid coincidences between the two primary detector arrays (CED and FPD), as well as whether or not a Čerenkov detector fired. A two-fold coincidence (CED-FPD detector pair hit plus no Čerenkov detector) classified the particle as a pion; a three-fold coincidence, which included a valid hit in a Čerenkov detector, classified the particle as an electron.

Fig. 5.2 shows the $G^{0}$ backward angle electron yield data for each of the target 



Figure 5.2: Normalized yield data in $H z / \mu A$ for each of the $G^{0}$ backward angle target and energy combinations. Data for only octant is shown. The vertical axis are the CEDs and the horizontal axis the FPDs. The detectors with the smallest number are located closest to the target. The cells outlined in black represents the elastic (quasi-elastic) locus. 
and energy combinations. The left-hand vertical axis represents the 9 CEDs in one octant, and the horizontal axis represents the same octant's 13 FPDs (numbered 3-16 as explained in Sec. 4.4). Each CED-FPD pair determines a "cell". The lowest detector numbers represent the detectors closest to the target. Yields and beam current were measured for each MPS. The numbers in the cells in Fig. 5.2 represent the cell's total rate, normalized using the beam current, in $H z / \mu A$, with its corresponding color shown on the right-hand vertical axis.

As previously mentioned, the CED-FPD pairs provided a coarse estimate of the scattered electron's momentum. Approximately 25 of the 117 CED-FPD cells are dominated by elastically scattered electrons. It is the asymmetry measured in these cells that are of interest, and the first step in obtaining this measurement is determining which cells comprise the "elastic (quasi-elastic) locus".

Figure 5.3 shows normalized yield versus SMS current settings [Mue09]. As the magnet's field strength was increased, higher momentum particles were moved toward the smaller CED and FPD detector numbers located closer to the target. These data were then fit using a Gaussian (blue) for the low momentum background and two Gaussians (with shared width in red) for the elastic peak. A constant (lt. green) was also added to the fit to remove any field independent rate. The results from these fits allowed a determination of the composition of particles detected in each cell during normal run conditions and are shown in Fig. 5.4 for each beam energy. This analysis laid the ground work for the dilution analysis described in Sec. 5.2.4.

The detector pairs that match the kinematics of elastically (quasi-elastically) 


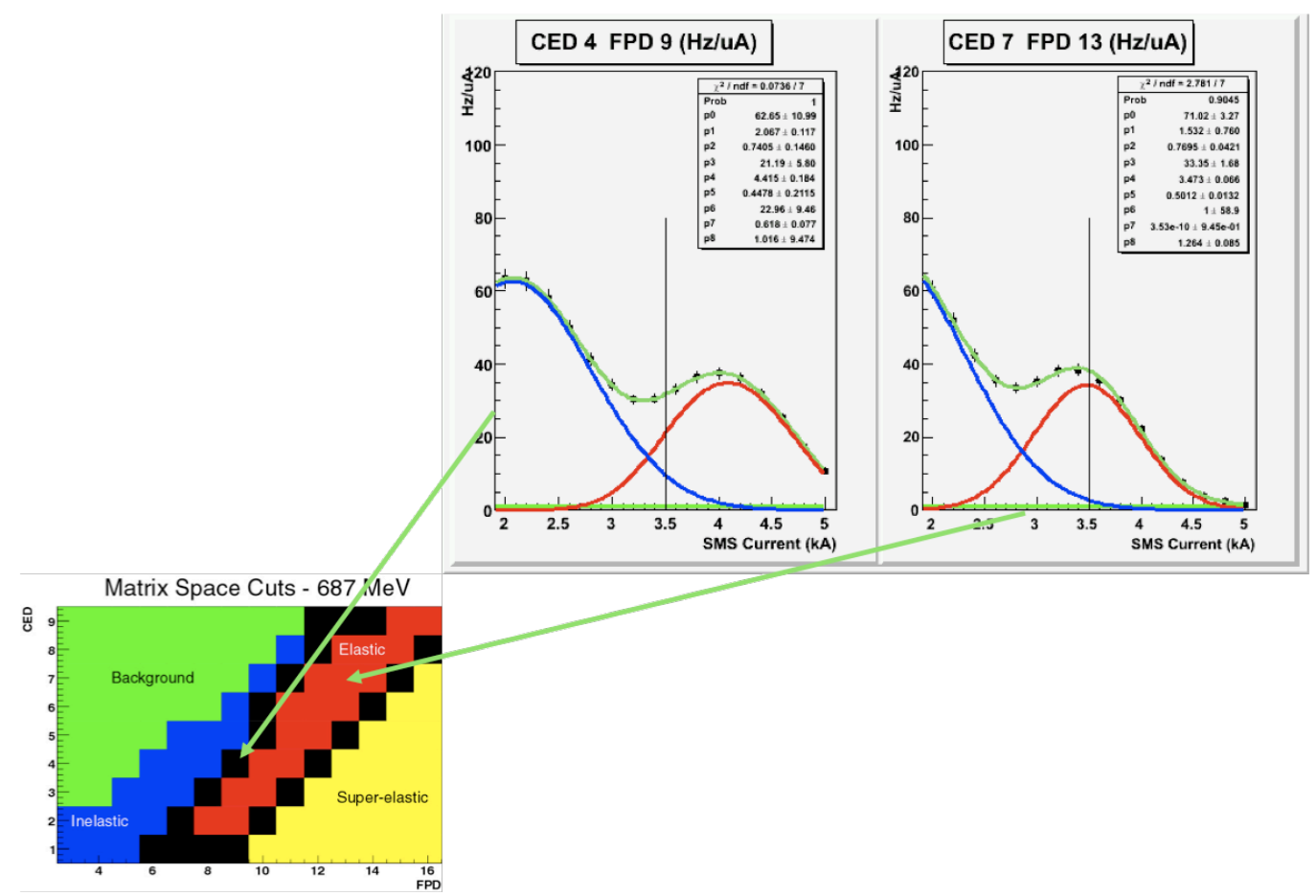

Figure 5.3: Cell by cell fits using a Gaussian (blue) for low momentum background and 2 Gaussians (with shared width) (red) for the elastic peak. A constant (lt. green) is also added to the fit to remove any field independent rate. The vertical black line is the nominal SMS setting. 
scattered electrons are shown in red. The detector pairs that only recorded inelastic electrons are shown in blue. The super-elastic region (yellow), are the detector pairs where it is kinematically impossible for scattered electrons to strike. The very low rate seen in those cells may be attributed to random coincidences or rescattering from parts of the target structure. These rates can be assumed to be a portion of the rate found in all of the cells, a heterogeneous distribution, and not restricted to just the superelastic region. The background region (green) contains a
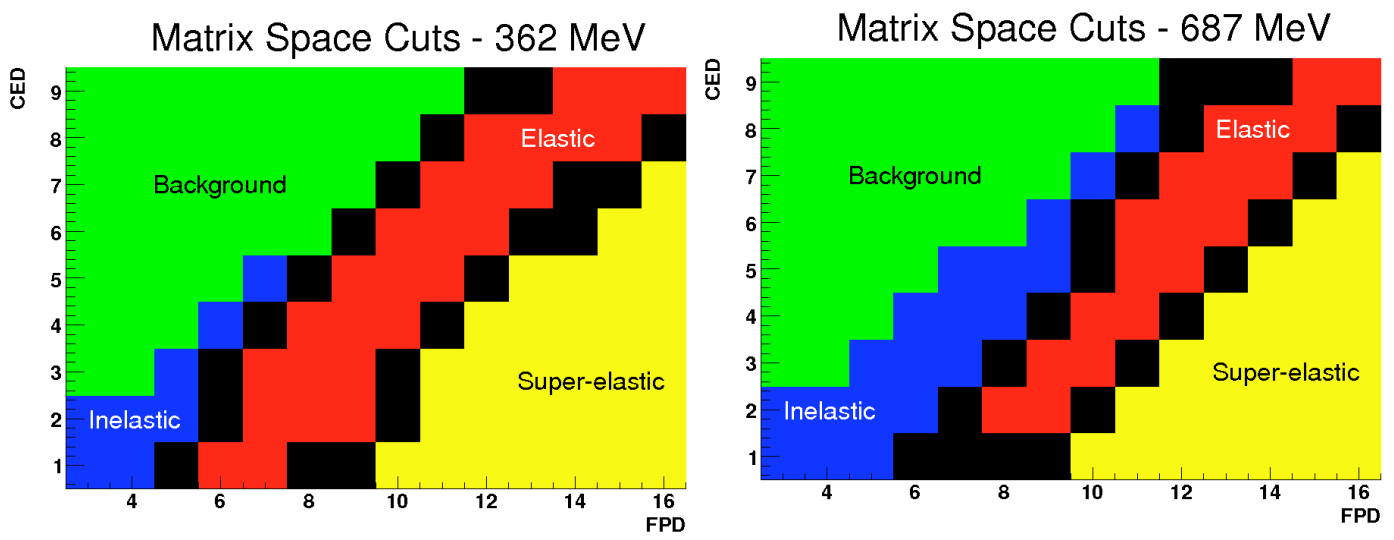

Figure 5.4: The various kinematic regions for the two beam energies as represented by CED - FPD detector pairs. CED number is on the vertical axis, FPD number on the horizontal axis. Red represents the cells dominated by elastic electrons, while the blue region is mostly inelastic electrons. Super-elastic is a region that should be inaccessible to electron scattering from the target, while the background region (green) is where various processes contribute to the rate, including those scattered from target walls. The black cells have a combination of particles from adjacent regions.

variety of particles including $\pi^{-}$, inelastically scattered electrons from the aluminum target walls as well as from the primary target, elastic (quasi-elastic) electrons that have radiated photons, and the occasional random detector coincidence that does not represent a real particle. The black cells are cells that contain a combination of particle types i.e., both elastic and inelastic electrons, and therefore have been 
excluded from any one matrix type.

An asymmetry was calculated for each quartet and then an average, weighted by the statistical error, of all of the quartets within each cell was calculated. Using this information, weighted averages of asymmetries from the cells that comprised the elastic (quasi-elastic) locus were made for each run and then for each target and energy combination. The blinded asymmetries prior to any corrections, versus octant are shown in Figs. 5.5 and 5.6 for each target and energy combination. The two states shown, "IN" and "OUT", refer to the state of the IHWP, which is discussed in Sec. 4.9.2. The asymmetry values shown are prior to any corrections and are blinded.

During the running of the experiment, the data were continuously checked for quality and for any indication of systematic effects. One of the ways that this was accomplished was through the use of the IHWP. The IHWP had two positions, "IN" and "OUT", which was changed approximately every three running days. If the asymmetry did not flip sign with a change in IHWP position, or if the value of OUT + IN was significantly different from zero, a systematic effect was probably the cause. The value for OUT - IN represents the weighted sum of the two helicity states.

Although the majority of the data analysis was performed by target type and beam energy, changes to the FPDs, discussed in Sec. 4.4, and Čerenkov detectors, discussed in Sec 4.6, resulted in multiple run periods for the high energy running. For the Čerenkov detectors, this was due in part to the photomultiplier tube change, but also because of a brief change in multiplicity (see Sec. 4.7). For the Fall 2006 


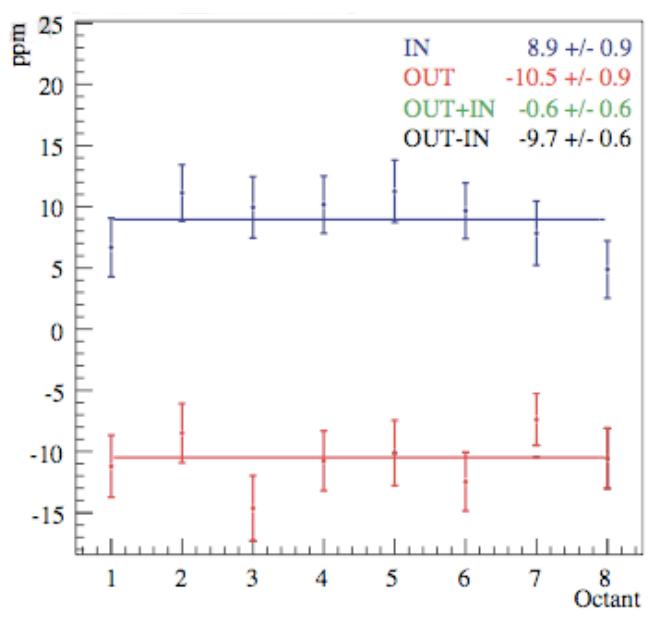

(a) $362 \mathrm{MeV}$

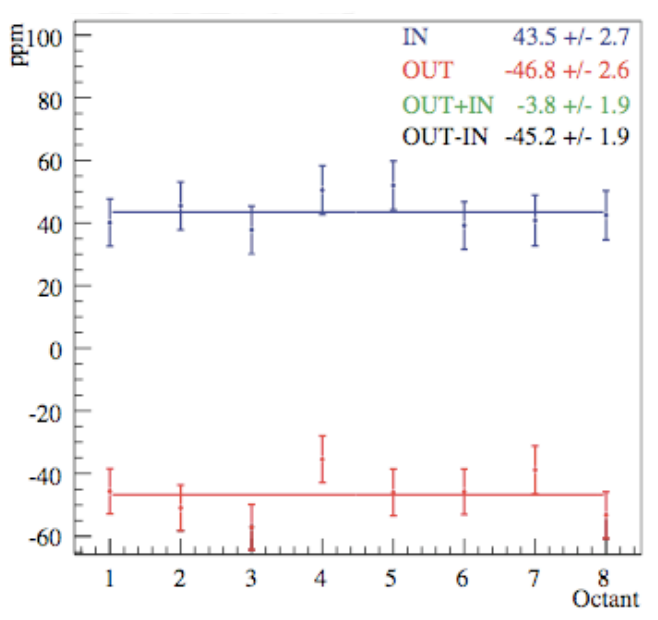

(b) $687 \mathrm{MeV}$

Figure 5.5: Typical asymmetry plots as a function of octant for electrons scattered elastically from a hydrogen target. No corrections have been made to the data, and the asymmetry values have the blinding factor applied.[Ver09]

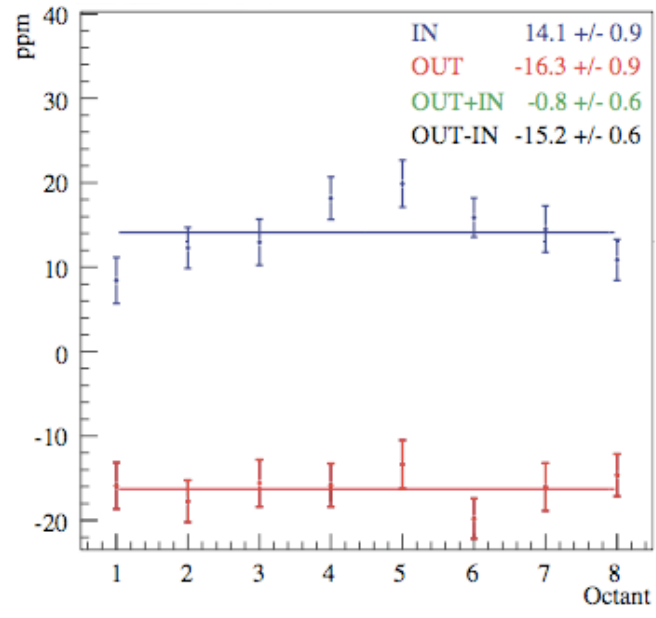

(a) $362 \mathrm{MeV}$

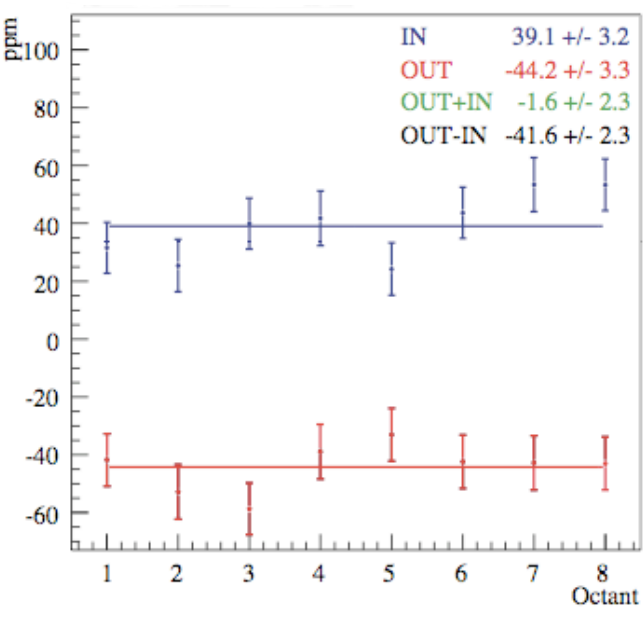

(b) $687 \mathrm{MeV}$

Figure 5.6: Typical asymmetry plots as a function of octant for electrons scattered quasi-elastically from a deuterium target. No corrections have been made to the data, and the asymmetry values have the blinding factor applied.[Ver09] 
data, the Čerenkov detector multiplicity was set at three, whereas for the remainder of the deuterium run periods, the multiplicity was set at two. For the final analysis, the normalized data for each target/beam energy were averaged together after the corrections were made for each run period. The run periods by target type and beam energy and current is shown in Table 5.2.

Table 5.2: The run periods showing target type, beam energy, and beam current.

\begin{tabular}{||c|c|c|c||}
\hline \hline Target & Beam Energy $(\mathrm{MeV})$ & Run Period & $I(\mu A)$ \\
\hline \hline H & 687 & April 2006 & 55 \\
H & 362 & Summer 2006 & 60 \\
H & 687 & Fall 2006 & 60 \\
D & 687 & Fall 2006 & 20 \\
D & 362 & Winter 2007 & 35 \\
D & 687 & March 2007 & 20 \\
\hline \hline
\end{tabular}

\subsection{Corrections to the Raw Asymmetries}

Fig. 5.7 shows the steps followed to take the raw and blinded measured asymmetries and produce values for the strange and axial vector form factors. The remainder of this chapter will describe the processes and methods necessary to determine $A_{\text {phys }}$, the corrected, physical asymmetry for each of the data sets. The next and final chapter of this thesis will describe how to combine $A_{\text {phys }}$ with the $G^{0}$ forward angle results to determine the strange and axial vector form factors at two momentum transfers. 


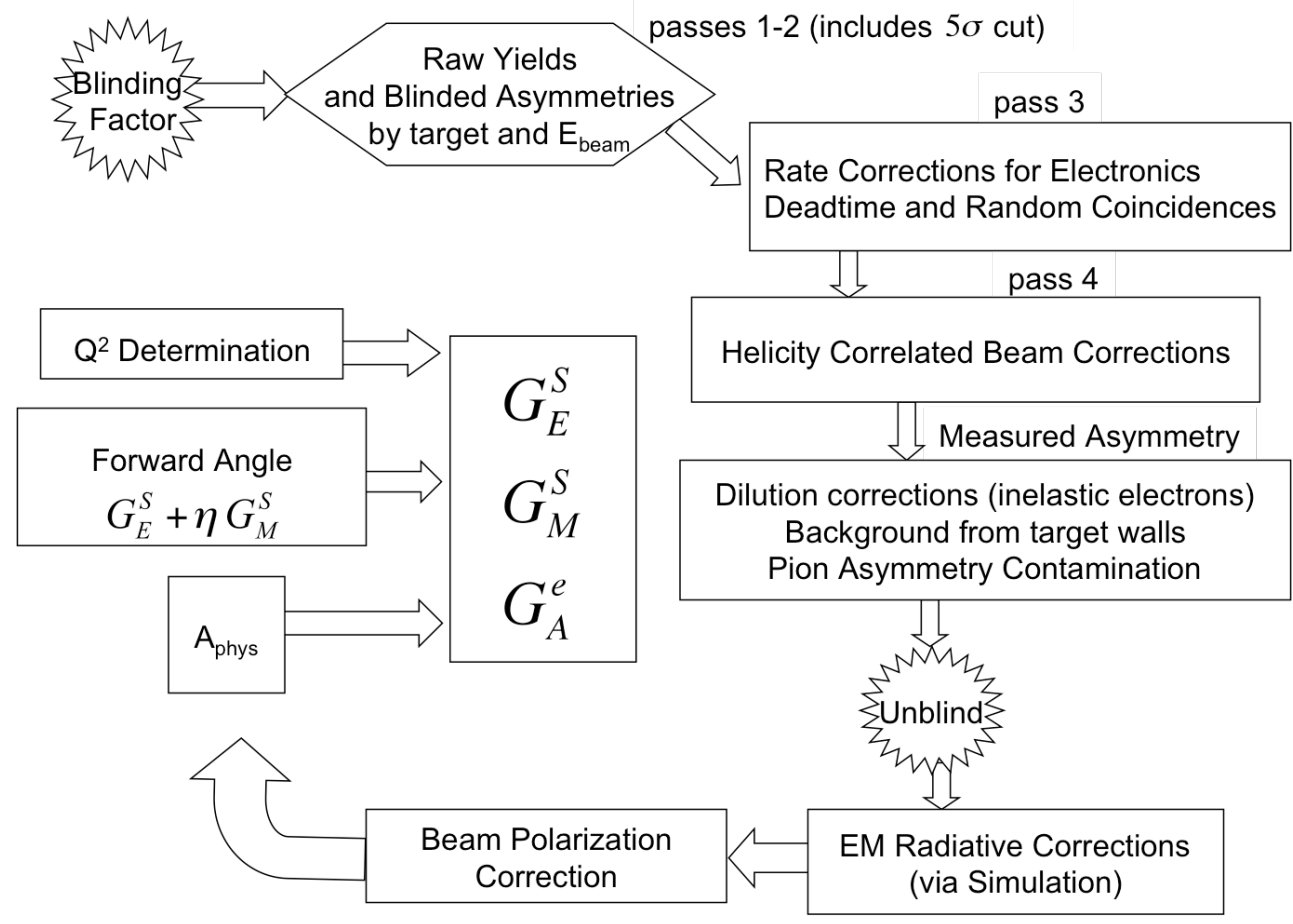

Figure 5.7: A diagram of the analysis process. A blinding factor is applied to the raw, uncorrected asymmetries. The raw yields, from which the raw asymmetries are formed, receives specific corrections during each of the 4 analysis "passes". After the 4th pass, the corrected yields generate the "measured" asymmetries, which are still blinded. The measured asymmetry is corrected for background events and dilutions before the blinding factor is removed. The electromagnetic radiative corrections and the correction for the beam polarization is applied to $A_{\text {meas }}$, generating $A_{\text {phy }}$, which is used to extract the vector strange and axial form factors. 


\subsubsection{Scaler Counting Rate Error Correction}

After the backward angle measurements began, it was apparent that for some of the North American scalers, there was a difference between the standard deviation and the width of the counting statistics distribution because a significant number

of events fell outside of a normal gaussian distribution, as shown in Fig. 5.9. Initial investigation showed that the problem only appeared in North American scalers recording a CED-FPD coincidence.

After a systematic analysis involving the problematic North American scalers, the properly performing French counterparts, and a simulation, the cause was determined. When a single channel in some of the North American scalers received two pulses that were too close together, the pulse pair would cause the scaler to drop or add a random bit. This only occurred if the pulses were narrow, and narrow pulses separated by more than 10 ns did not cause a problem. The purpose of these scalers was to record a coincidence between a CED mean timer and an FPD mean timer, when the two signals were also in coincidence with a common trigger signal. The trigger module had a dead time of $\approx 15 \mathrm{~ns}$, the meantimer dead time was $\approx 20 \mathrm{~ns}$. The original programming of the coincidence modules formed output pulses as the logical AND of the CED-FPD signals and the trigger. This could allow two narrow and closely spaced signals to be counted within the same trigger and mean timer windows. For example, as shown in Fig. 5.8, if two mean timer FPD signals separated by 2 ns where both within the trigger window of a CED mean timer signal, two separate signals that were each 7 ns long would be sent to 


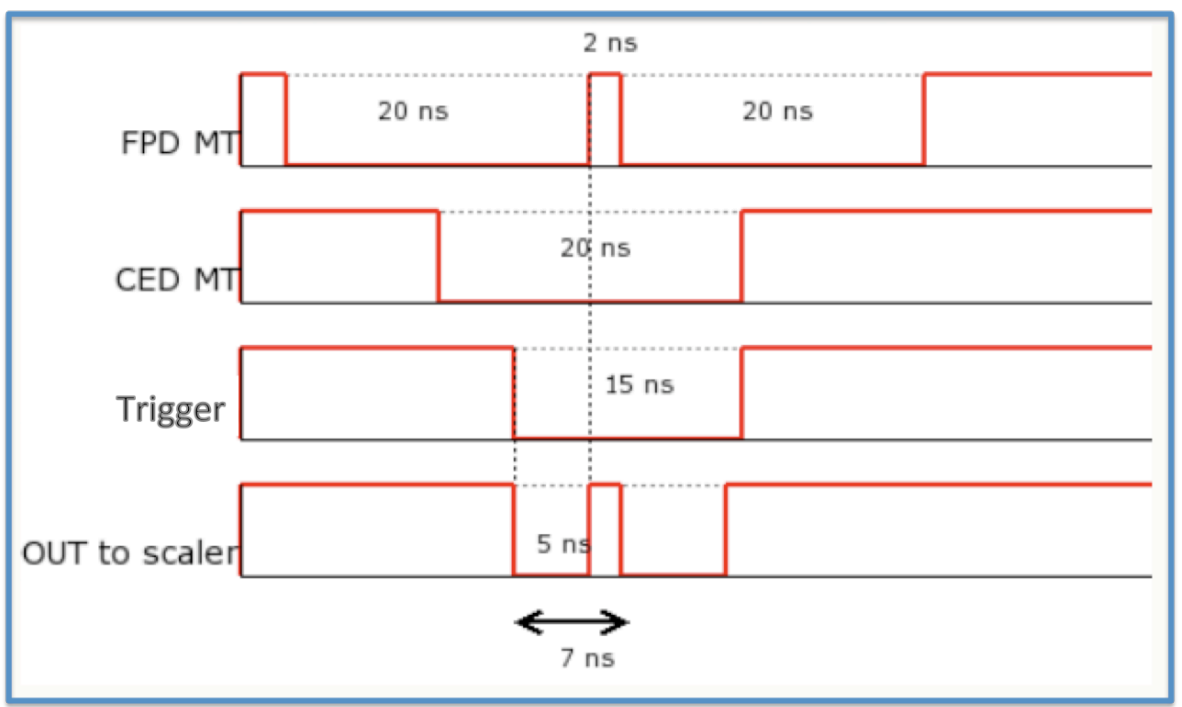

Figure 5.8: The cause of the bit error in the scaler that recorded a CED-FPD coincidence. If two mean timer FPD signals separated by 2 ns were within the trigger window of a CED mean timer signal, two 7 ns pulses would be sent to the scaler, instead of one 10 ns signal resulting in a complicated pattern of bit inversion errors [Rea08].

the scaler, instead of one 10 ns signal. The double-signal resulted in a complicated pattern of unintended bit flipping in the scaler modules.

Once the problem and its cause were discovered, the North American electronics were reprogramed to fix the length of the scaler intput signal to $10 \mathrm{~ns}$. In order to use the data that had already been gathered, a study was conducted to determine the efficacy and the effect of applying a cut on the yield data [Cap08]. It was determined that placing a cut on all of the scalers that would remove events that were $5 \sigma$ or more away from the mean distribution was the best course of action. The cuts were only made on the yield data, not on the asymmetry. For the French scalers, less than $0.08 \%$ of the MPS were cut. For the North American scalers, approximately $0.14-0.35 \%$ of the MPS were rejected, depending upon the data set 


\section{Electron coincidence yield CED 9-FPD 16 octant3}

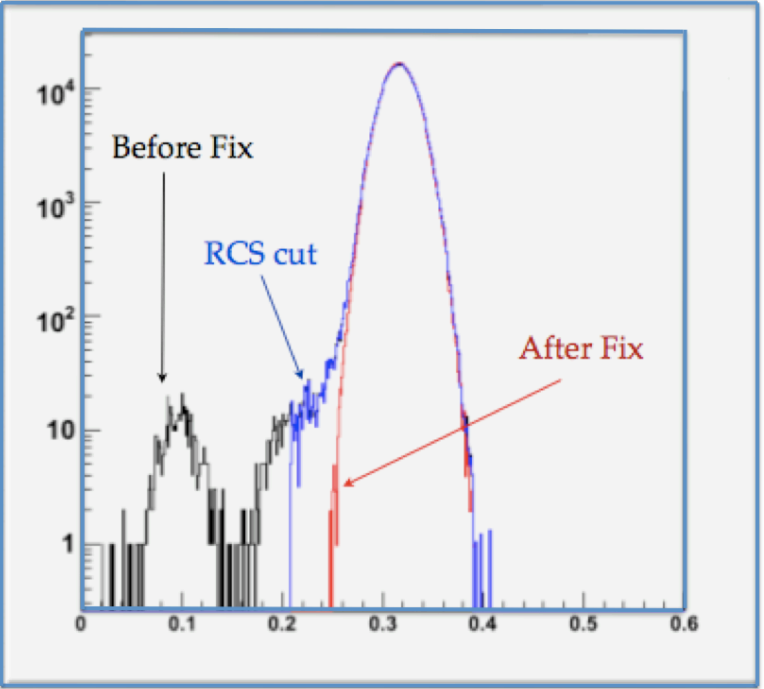

Figure 5.9: The plot shows the yield in $k H z / \mu A$ for one CED-FPD detector pair during the low energy running with a deuterium target. The non-Gaussian distribution shown in black and blue around the mean yield, indicates a problem with the electronics. The result of cutting all events $5 \sigma$ or more away from the mean distribution is shown in red. The cut is on the yield and no cuts were made to the asymmetries.

[Fur09].

The systematic error induced by the scaler counting error can be estimated based on the asymmetry associated with the bad events that remained after the $5 \sigma$ cut was made. This process was first presented in [Fur09] and expanded upon in [Ver09]. The result of the calculation was that the error associated with the scaler counting error after the $5 \sigma$ cut correction was made, was less than $0.001 \mathrm{ppm}$, and was therefore neglected.

\subsubsection{Electronics Rate Correction}

Both electron and pion rates and their associated asymmetries were measured using the electronics and detectors from the $G^{0}$ backangle measurement. To obtain 
the true rate for both of these particles, corrections to account for effects due to the electronics were required. An overview of the process used to determine the rate corrections, along with rate-related residual false asymmetries and associated errors are presented in this section. For a detailed explanation of the electronics rate corrections, see [Ver09]:

The rate correction associated with the electronics has three components. They are:

- Electronics dead time correction: This corrects for any valid events lost due to the recovery time of any module within the electronics chain. This correction will increase the true rate as compared to the measured rate.

- Randoms: This correction removes accidental detector coincidences. This correction will lower the true rate as compared to the measured rate.

- Contamination: This correction corrects for pions misidentified as electrons and vice versa, which will produce a true rate lower than the measured rate.

These three components of the electronics rate corrections were applied to the $G^{0}$ backward angle data in two steps. First, the rates associated with dead time and randoms that involved all of the electronics modules associated with $\mathrm{CED} \times \mathrm{FPD}$ coincidences were calculated and applied, producing the associated corrected rate, $\tilde{r}_{D T c o r r}$. Secondly, a final rate correction was applied that modified $\tilde{r}_{D T c o r r}$ to account for the Cerenkov electronics. Those corrections included a rate adjustment due to Čerenkov electronics dead time as well as a correction for pions misidentified as electrons. All of the rate corrections are applied MPS by MPS. The rest of 
this section details the two step rate correction process, which upon completion, produced the final true electron and pion rates.

The relationship between the beam normalized measured rate, $\tilde{r}_{m e a s}$, and the true rate, $\tilde{r}_{\text {true }}$, is

$$
\tilde{r}_{\text {meas }}=(1-D T) \tilde{r}_{\text {true }}
$$

where DT is the probability that at least one event will arrive during the recovery time, or the dead time, of the electronics. For each module in the electronics chain, DT is

$$
D T=1-e^{-\tau I \tilde{r}_{m e a s}},
$$

where $I$ is the beam current and $\tau$ is the recovery time the electronics requires between events. In the case of the $G^{0}$ backward angle measurement, [because $\tau<1$ second and $\tilde{r}_{\text {meas }}$ is of the order of $\mathrm{MHz}$, Eqn. 5.5 can be simplified to

$$
D T \approx \tau I \tilde{r}_{\text {meas }}
$$

For a CED $\times$ FPD coincidence, the modules that impact the true versus measured rate due to their intrinsic dead time are the CFDs, the mean timers (MT) for both the CED and FPD detector arrays, and the coincidence trigger.

The rate of the random events associated with the CEDs and FPDs is [Ver09]:

$$
\tilde{r}_{\text {rand }}=\left(\tilde{r}_{M T_{i}}-\tilde{r}_{\text {coinc }}\right) \times\left(\tilde{r}_{M T_{j}}-\tilde{r}_{\text {coinc }}\right) \times \Delta_{\text {Trig }} \times I
$$

where the indices $i$ and $j$ designate a detector pair $\left(\mathrm{CED}_{i}, \mathrm{FPD}_{j}\right), \Delta_{\text {Trig }}$ is the width of the trigger window, and $\tilde{r}_{\text {coinc }_{i(j)}}$ corresponds to the normalized MT rate for events associated with a CED $\times$ FPD coincidence. It should be noted that the coincidence 
rate only includes those events where one and only one MT FPD and one MT CED were in coincidence. Multiple coincidences, also called multi-hits (MH), involve more than two MT signals in coincidence and were not considered "valid" coincidences, by the electronics logic. However, multi-hits can induce a loss of signal due to associated electronics dead time, and must therefore be included in the overall rate correction.

The measured rate shown below is a combination of all of the effects associated with random coincidences and electronics dead time related to the $\mathrm{CED} \times \mathrm{FPD}$ coincidence electronics [Ver09],

$$
\begin{array}{r}
\tilde{r}_{\text {meas }}^{ \pm}=\left[\tilde{r}_{D T c o r}^{ \pm}\left(1-D T_{C F D}^{c e d}-D T_{M T}^{c e d}\right)\left(1-D T_{C F D}^{f p d}-D T_{M T}^{f p d}\right)\right. \\
\left.+\tilde{r}_{\text {rand }}\right]\left(1-D T_{\text {Trig }}-M H_{22}\right) \overline{M H_{12}},
\end{array}
$$

where $\tilde{r}_{D T \text { cor }}^{ \pm}$represents the $\mathrm{CED} \times \mathrm{FPD}$ coincidence rate corrected for electronics dead time while $\tilde{r}_{\text {rand }}$.

The electronics rate correction accounts for coincidences lost due to CFD dead time $\left(D T_{C F D}^{f p d(c e d)}\right)$, MT dead time $\left(D T_{M T}^{c e d(f p d)}\right)$, trigger dead time $\left(D T_{\text {Trig }}\right)$ as well as coincidences lost due to electronics dead time from multi-hits $\left(\mathrm{MH}_{12}\right.$ and $\left.\mathrm{MH}_{22}\right)$. It includes random coincidences between two uncorrelated MTs $\left(\tilde{r}_{\text {rand }}\right)$ and also accounts for losses to the random rate due to electronics dead time. The CFD and MT dead times are not applied to the randoms because measured MT rates were used to calculate the randoms [Pil07]. The actual values for $D T_{l}^{m}$ are more complicated than Eqn. 5.6 because of the correlations between the CFDs, MTs, and the trigger. The detailed expressions for these values, as well as the expressions 
for the MH parameters, are available in [Pil07] while the actual values used for the correction are in [Ver09].

It should be emphasized that depending on the location of the cell, the rate correction for the $\mathrm{CED} \times \mathrm{FPD}$ coincidence electronics may raise or lower the true rate in comparison to the measured rate. This can be seen clearly in Fig. 5.10

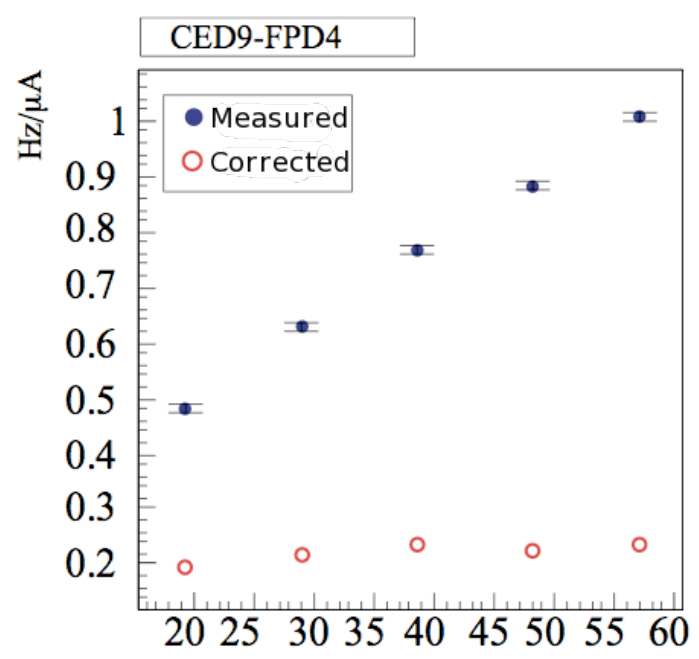

(a) Cell dominated by randoms.

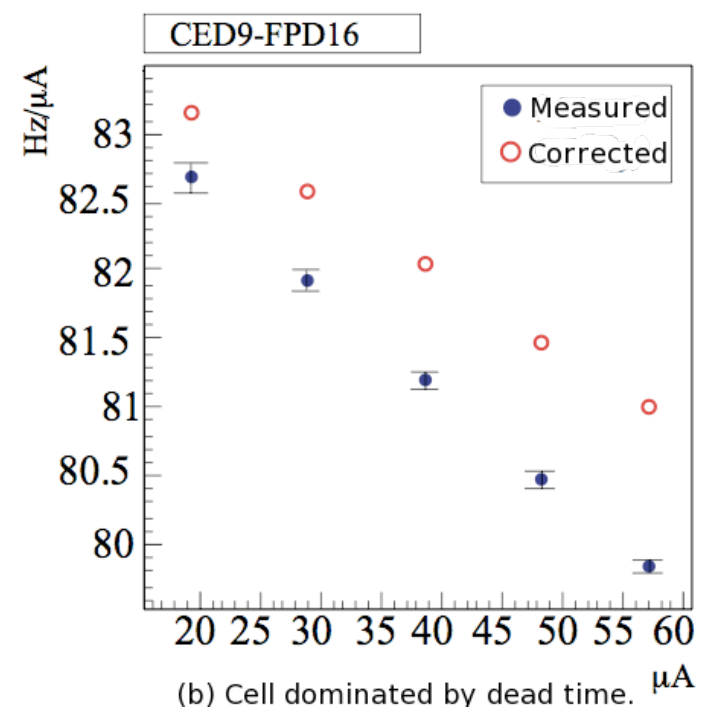

Figure 5.10: The measured and corrected rate for $e+\pi$ (all CED $\times$ FPD coincidences with or without a Čerenkov hit) versus beam current. The corrections account for electronics dead time and random events. The data were from a hydrogen target at $687 \mathrm{MeV}$ for two cells affected primarily by one correction or the other. The cell on the left is in the background region. The cell on the right is in the elastic region. [Ver09]

where the $\mathrm{CED} \times \mathrm{FPD}$ coincidence rates are shown (this includes both pions and electrons) as a function of beam current. The cell on the left is from the background region and is dominated by random coincidences. When the rate correction is applied, the total rate is lowered. The constant slope seen after the correction demonstrates the quality of this correction. The cell on the right is in the elastic region and the rate correction that dominates is that due to electronics dead time, 
so the corrected rate is higher than the measured rate. The small slope of the corrected data for this cell is indicative of residual effects that were uncorrected. This was accounted for when the systematic error was calculated [Ver09].

The preceding implies that the rate correction up to this point was the same for both electrons and pions, which was actually not the case. The random coincidence correction requires knowledge of the fraction of MTs associated with a Čerenkov trigger and this information was not available for the North American octants. Additionally, it was assumed that the probability of having a CED $\times$ FPD $\times$ Čerenkov random was low. Therefore, all CED $\times$ FPD randoms were subtracted from the pion rate. For the electrons, the false asymmetry associated with CED $\times$ FPD randoms was determined and was subtracted from the value for $A_{\text {calc }}$ so that $A_{\text {true }}=A_{\text {calc }}-A_{\text {false }} \pm \epsilon_{\text {tot }}$ where $\epsilon$ is the total error associated with the rate correction.

The final part of the rate correction accounts for dead time and random coincidences in the Cerenkov electronics. If an electron makes a good CED $\times$ FPD coincidence, but dead time prevents the Čerenkov from firing, then an electron is improperly classified as a pion. Conversely, if a pion is accompanied by a random firing of the Čerenkov, then a pion is misidentified as an electron. Thus, the total corrected coincidence rate, $\tilde{r}_{D T c o r}$, does not change, but the ratio of electrons to pions does change.

The electronics rate correction was made by first inverting Eqn. 5.8 to produce 
a value for $\tilde{r}_{D T c o r}$, and then the following coupled equations were solved [Ver09]:

$$
\begin{aligned}
& \tilde{r}_{D T c o r}^{e}=\tilde{r}_{\text {calc }}^{e}\left(1-D T_{c}\right) \tilde{r}_{\text {calc }}^{\pi}+R_{\text {ran }}^{c}, \\
& \tilde{r}_{D T c o r}^{\pi}=\tilde{r}_{\text {calc }}^{e} D T_{c}+\tilde{r}_{\text {calc }}^{\pi}\left(1-R_{\text {ran }}^{c}\right) .
\end{aligned}
$$

The values for the Čerenkov dead time, $D T_{c}$, and Čerenkov random events, $R_{\text {ran }}^{c}$, were measured and vary with data set and are recorded in [Ver09].

As a reminder, the electronics rate corrections were applied MPS by MPS to each of the cells in the CED/FPD detector matrix. To provide a sense of the size of the correction, Table 5.3 summarizes the total percent change in the normalized rate of elastic (quasi-elastic) electrons by octant, for a typical run in each data set.

The negative correction for the LD2 $687 \mathrm{MeV}$ data set is because those data were

Table 5.3: Correction applied to the normalized measured rate of electrons in the

\begin{tabular}{|c|c|c|c|c|c|c|c|c|}
\hline \multirow{3}{*}{ Data Set } & \multicolumn{8}{|c|}{ Octant Number } \\
\hline & 1 & 2 & 3 & 4 & 5 & 6 & 7 & 8 \\
\hline & \multicolumn{8}{|c|}{ Change in Rate $(\% \mathrm{~Hz} / \mu \mathrm{A})$} \\
\hline 362 & 11 & 5 & 12 & 5 & 12 & 5 & 1 & 5 \\
\hline 362 & 10 & 5 & 10 & 4 & 9 & 5 & 9 & 4 \\
\hline H 687 & 6 & 2 & 7 & 3 & 6 & 3 & 5 & 3 \\
\hline $\mathrm{D} \quad 687$ & -14 & -8 & -18 & -3 & -7 & -5 & -18 & -7 \\
\hline
\end{tabular}
elastic or quasi-elastic region as a function of octant [Ver09].

dominated by pions and the amount of random Čerenkov firings in coincidence with a good $\mathrm{CED} \times$ coincidence was greater than the number of events lost due to Cerenkov dead time. This also shows that the French (even numbered) octants required a consistently lower rate correction than the North American (odd numbered) octants. 
This was due to inherent differences in the electronics [Ver09].

The rate corrections were calculated and applied using the $G^{0}$ replay engine, in which the asymmetry was constructed

$$
A_{\text {calc }}=\frac{\tilde{r}_{\text {calc }}^{+}-\tilde{r}_{c a l c}^{-}}{\tilde{r}_{\text {calc }}^{+}+\tilde{r}_{c a l c}^{-}},
$$

and stored in the $G^{0}$ database.

The difference in the size of the asymmetry when the rate corrections are applied, as compared to when they are not, were compared. This difference, $A_{\text {sys }}$, was used to validate the rate correction used in the $G^{0}$ replay engine and it was also used to help quantify the size of the false asymmetry associated with the rate corrections. It can be expressed as [Ver09]:

$$
\begin{aligned}
A_{\text {sys }} & =A_{\text {meas }}-A_{\text {calc }} \\
& =A_{\text {single }}+A_{\text {trig }}+A_{M H_{12}}+A_{\text {cer }} .
\end{aligned}
$$

The term $A_{\text {single }}$ represents the asymmetry associated with the dead time of the CFDs and MTs, $A_{\text {trig }}$ represents a similar value for the dead time associated with the $\mathrm{CED} \times \mathrm{FPD}$ coincidence trigger and multiple coincidences $\left(\mathrm{MH}_{22}\right), A_{M H_{12}}$ represents the same for multi-hits $\left(\mathrm{MH}_{12}\right)$, and $A_{c e r}$ is associated with contamination due to the Čerenkov electronics. The values in Eqn. 5.13 were determined analytically [Ver09] and compared to the values computed in the $G^{0}$ replay engine using 
Eqn. 5.12. For electrons,

$$
\begin{aligned}
A_{\text {single }}= & -\left[\frac{D T_{M T}^{c e d}}{1-D T_{C F D}^{c e d}-D T_{M T}^{c e d}} A\left(D T_{M T}^{c e d}\right)+\frac{D T_{C F D}^{c e d}}{1-D T_{C F D}^{c e d}-D T_{M T}^{c e d}} A\left(D T_{C F D}^{c e d}\right)\right. \\
& \left.+\frac{D T_{M T}^{f p d}}{1-D T_{C F D}^{f p d}-D T_{M T}^{f p d}} A\left(D T_{M T}^{f p d}\right)+\frac{D T_{C F D}^{f p d}}{1-D T_{C F D}^{f p d}-D T_{M T}^{f p d}} A\left(D T_{C F D}^{f p d}\right)\right] \\
A_{\text {Trig }}= & -\frac{D T_{\text {Trig }}}{1-D T_{\text {Trig }}-M H_{22}} A\left(D T_{\text {Trig }}\right)-\frac{M H_{22}}{1-D T_{\text {Trig }}-M H_{22}} A\left(M H_{22}\right) \\
A_{M H_{12}}= & -\frac{M H_{12}}{1-M H_{12}} A\left(M H_{12}\right) \\
A_{\text {cer }}= & f_{\pi}\left(A_{\text {true }}^{\pi}-A_{\text {true }}^{e}\right)-\left(1-f_{\pi}\right) \frac{D T_{c}}{1-D T_{c}} A\left(D T_{c}\right)+f_{\pi} A\left(R_{\text {ran }}^{c}\right)
\end{aligned}
$$

where the contribution to the asymmetries from the CFD/MT dead time probability are $A\left(D T_{M T}^{c e d}\right), A\left(D T_{C F D}^{c e d}\right), A\left(D T_{M T}^{f p d}\right)$, and $A\left(D T_{C F D}^{c e d}\right)$, and $A\left(D T_{C F D}^{c e d}\right)$, and $f_{\pi}$ is the fraction of pions misidentified as electrons. The contribution to the asymmetry from the probability of Čerenkov random coincidence is $A\left(R_{r a n}^{c}\right)$. The largest contribution to the false asymmetry is from pion contamination, the first term in $A_{c e r}$.

There were three residual effects not accounted for by the rate correction in the $G^{0}$ replay engine. These were:

1. Unmeasured events below the threshold set for the CFDs that would have generated an unaccounted for dead time.

2. A failure to correct for CFD dead time in the French octants due to a scaler counting problem.

3. The subtraction of random CED $\times$ FPD coincidences were exclusively applied to the pion data because it was impossible to correlate the CED $\times$ FPD coincidences with a Čerenkov signal. 
Table 5.4: Final false asymmetry and systematic error assigned to the electronics rate correction for elastic (quasi-elastic) electrons shown for each data set [Ver09].

\begin{tabular}{|c|c|c|c|c|c|c|}
\hline \multicolumn{2}{|r|}{ data set } & IHWP & $\begin{array}{l}A_{\text {false }} \\
(\mathrm{ppm})\end{array}$ & $\begin{array}{c}\epsilon_{\text {stat }} \\
(\mathrm{ppm})\end{array}$ & $\begin{array}{c}\epsilon_{\text {resid }}^{D T} \\
\text { (ppm) }\end{array}$ & $\begin{array}{c}\epsilon_{\text {resid }}^{r d m} \\
\text { (ppm) }\end{array}$ \\
\hline \multirow[t]{2}{*}{ LH2 } & 362 & in & -0.00 & 0.04 & 0.06 & 0.00 \\
\hline & & out & 0.00 & 0.04 & 0.07 & 0.00 \\
\hline \multirow[t]{2}{*}{ LH2 } & 687 & in & -0.15 & 0.16 & 0.06 & 0.02 \\
\hline & & out & 0.15 & 0.16 & 0.07 & 0.02 \\
\hline \multirow[t]{2}{*}{ LD2 } & 362 & in & -0.04 & 0.20 & 0.02 & 0.01 \\
\hline & & out & 0.04 & 0.21 & 0.04 & 0.01 \\
\hline \multirow[t]{2}{*}{ LD2 } & 687 (March 2007) & in & -0.35 & 1.19 & 0.01 & 0.08 \\
\hline & & out & 0.39 & 2.17 & 0.01 & 0.10 \\
\hline \multirow[t]{2}{*}{ LD2 } & (Fall 2006) & in & -0.27 & 1.77 & 0.00 & 0.10 \\
\hline & & out & 0.43 & 1.60 & 0.00 & 0.14 \\
\hline
\end{tabular}

The final systematic error for the rate corrections associated with the electronics for elastic (quasi-elastic) electrons, is shown in Table 5.4 by data set and for each IHWP setting. The false asymmetry, $A_{\text {false }}$, is the asymmetry associated with the random $\mathrm{CED} \times \mathrm{FPD}$ coincidences that were "electron-like" but that were not subtracted from the electron rate, because as previously mentioned, the electronics did not allow a determination of which randoms were "electron-like" and which were "pion-like" so all CED $\times$ FPD coincidence randoms were subtracted from the pions. The error associated with this false asymmetry is $\epsilon_{\text {resid }}^{r d m}$. The statistical uncertainty is $\epsilon_{\text {stat }}$ and the uncertainty associated with the uncorrected CFD residual dead time is $\epsilon_{\text {resid }}^{D T}$.

Table 5.5 gives the resulting change to the asymmetry and the associated uncertainty after the correction for the electronics was applied to the yields. 
Table 5.5: Corrections to the raw measured asymmetry due to corrections to the yield to account for electronics effects for each target and beam energy along with the associated uncertainty $\left[\mathrm{Aac}^{+} 10\right]$.

\begin{tabular}{||c|c|c||}
\hline \hline Target & $\begin{array}{c}\text { Beam Energy } \\
(\mathrm{MeV})\end{array}$ & $\Delta A_{D T}(\mathrm{ppm})$ \\
\hline \hline $\mathrm{H}$ & 362 & $-0.31 \pm 0.08$ \\
$\mathrm{D}$ & 362 & $-0.58 \pm 0.21$ \\
$\mathrm{H}$ & 687 & $-1.28 \pm 0.18$ \\
$\mathrm{D}$ & 687 & $-7.0 \pm 1.8$ \\
\hline \hline
\end{tabular}

\subsubsection{Linear Regression}

Linear regression corrects false asymmetries caused by helicity-correlated beam parameter differences. A standard linear regression analysis was performed for the $G^{0}$ backward angle measurement which was similar to that completed for the forward angle data set, and used for most experiments where a parity-violating asymmetry measurement is made. Details of this analysis can be found in [Nak05] and [Liu06]; an overview of the process is described below.

When the beam is on, the characteristics of the beam will vary around mean values. These variations may impact the yield seen in the detectors and if these variations are helicity-correlated, they can be the source of a false asymmetry. Great effort is expended to minimize any helicity-correlated effect, and when the effect can not be eliminated, the final asymmetry requires a correction. The beam parameters of interest are $\mathrm{X}, \mathrm{Y}, \theta_{x}, \theta_{y}$, E, and $\mathrm{Q}$ which are the horizontal and vertical beam positions and angles, the beam energy, and the beam charge. The four position parameters completely describe the beam position on target. Table 5.6 shows the 
helicity-correlated properties for the $G^{0}$ backward angle measurement; both the maximum allowed difference or asymmetry [CPP05], and the actual difference. In all cases, the actual helicity-correlated difference was much lower than the requirement.

Table 5.6: The measured and maximum allowed charge asymmetry or beam parameter difference for the $G^{0}$ backward angle measurement.

\begin{tabular}{||c|c|c||}
\hline \hline Beam Parameter & Achieved & Requirement \\
\hline \hline Charge asymmetry $\left(\left(I^{+}-I^{-}\right) /\left(I^{+}+I^{-}\right)\right)$ & $0.09 \pm 0.08$ & $2 \mathrm{ppm}$ \\
x position $\left(x^{+}-x^{-}\right)$ & $-19 \pm 3$ & $40 \mathrm{~nm}$ \\
y position $\left(y^{+}-y^{-}\right)$ & $-17 \pm 2$ & $40 \mathrm{~nm}$ \\
x angle $\left(\theta_{x}^{+}-\theta_{x}^{-}\right)$ & $-0.8 \pm 0.2$ & $4 \mathrm{nrad}$ \\
y angle $\left(\theta_{y}^{+}-\theta_{y}^{-}\right)$ & $0.0 \pm 0.1$ & $4 \mathrm{nrad}$ \\
Energy $\left(E^{+}-E^{-}\right)$ & $2.5 \pm 0.5$ & $34 \mathrm{eV}$ \\
\hline \hline
\end{tabular}

To correct for these very small but real effects, consider the yield as composed of two values, one completely independent from helicity-correlated beam parameter effects $\left(Y_{P V}^{ \pm}\right)$and the other comprised of the yield contributions due to correlations with the beam parameters:

$$
Y^{ \pm}=Y_{P V}^{ \pm}+\sum_{i=1}^{6} \alpha_{i} \delta x_{i}^{ \pm},
$$

where $Y^{+(-)}$is the measured normalized yield for the positive (negative) helicity state, $i$ is the index of the beam parameter, $\alpha_{i}=\partial Y / \partial x_{i}$ is the slope of the normalized yield versus a beam parameter, and $\delta x_{i}^{ \pm}=x_{i}^{ \pm}-\left\langle x_{i}^{ \pm}\right\rangle$is the deviation of the beam parameter, $x_{i}$, from its average over a run, $\left\langle x_{i}\right\rangle$.

As previously stated, the yield measurements from both helicity states determine the asymmetry, 


$$
\begin{aligned}
A_{\text {meas }} & =\frac{Y^{+}-Y^{-}}{Y^{+}+Y^{-}} \\
& =\frac{Y_{P V}^{+}-Y_{P V}^{-}+\sum_{i}\left(x_{i}^{+}-x_{i}^{-}\right)}{Y_{P V}^{+}+Y_{P V}^{-}+\sum_{i}\left(x_{i}^{+}+x_{i}^{-}-2\left\langle x_{i}\right\rangle\right)} \\
& \simeq A_{P V}+\frac{\alpha_{i} \Delta x_{i}}{2\langle Y\rangle},
\end{aligned}
$$

where $\Delta x_{i}=x_{i}^{+}-x_{i}^{-}$and $\langle Y\rangle=\frac{1}{2}\left(Y_{P V}^{+}+Y_{P V}^{-}\right)$. Both $\langle Y\rangle$ and $\Delta x_{i}$ were calculated during the running of the experiment so the false asymmetry due to helicitycorrelated beam parameters, $\left(\alpha_{i} \Delta x_{i} / 2\langle Y\rangle\right)$, can be calculated once the correlation slopes, $\alpha_{i}$, are determined.

Revisiting Eqn. 5.15, the net change of the yield due to helicity-correlated effects is

$$
\delta Y=\sum_{i}^{6} \alpha_{i} \delta x_{i} .
$$

The correlation slopes can be found using a $\chi^{2}$ multi-dimensional least-squares method [BR03] where

$$
\chi^{2}=\sum_{j} \frac{\left(\delta Y^{j}-\sum_{i} \alpha_{i} \delta x_{i}^{j}\right)^{2}}{\left(\sigma^{j}\right)^{2}},
$$

with $\sigma_{j}^{2}$ the uncertainty of $\delta Y^{j}-\sum_{i} \alpha_{i} \delta x_{i}^{j}$ for measurement $j$. Taking $\partial \chi^{2} / \partial \alpha_{i}=0$, assuming that all measurements carry the same statistical weight, i.e. $\sigma_{i}=\sigma$, and replacing $\sum_{j}$ with the straight average of all measurements, results in the following expression

$$
\left\langle\delta x_{k} \delta Y\right\rangle=\sum_{i} \alpha_{i}\left\langle\delta x_{k} \delta x_{i}\right\rangle
$$

The vector, $\left\langle\delta x_{k} \delta Y\right\rangle$, is the covariance between the beam parameters and the yields and $\left\langle\delta x_{k} \delta x_{i}\right\rangle$ is a $6 \times 6$ matrix representing the correlation between the beam pa- 
rameters. Using the following relationship,

$$
\begin{aligned}
\langle\delta x \delta y\rangle & =\langle(x-\bar{x})(y-\bar{y})\rangle \\
& =\langle x y-x \bar{y}-\bar{x} y+\bar{x} \bar{y}\rangle=\langle x y\rangle-\langle x\rangle\langle y\rangle
\end{aligned}
$$

the covariance terms were computed run-by-run using the $G^{0}$ replay engine, along with average beam parameters, yields, and their products. Once the covariance terms were computed, the slopes were determined simply by inverting the $6 \times 6$ matrix in Eqn. 5.19. Fig. 5.11 shows the slopes as a function of run number for the low energy data and hydrogen target. All slopes are very small and consistent with zero over time.

The statistical uncertainty of the slopes is determined using the curvature matrix of $\chi^{2}$. The error matrix, $\epsilon$, is the inverse of the curvature matrix, $C_{l k}$, which in the case of $\chi^{2}$ is the second cross-partial derivative with respect to two independent correlation slopes $\alpha_{l}$ and $\alpha_{k}$, [BR03]

$$
C_{l k}=\frac{1}{2} \frac{\partial^{2} \chi^{2}}{\partial c_{l} \partial C_{k}}=\frac{N\left\langle\delta x_{l} \delta x_{k}\right\rangle}{\sigma^{2}}
$$

where $\mathrm{N}$ is the total number of measurements. Recall that the full analysis of the $G^{0}$ backward angle data involved four replays of the data. The only difference between the third and fourth data passes was that the slopes calculated in the third pass were used to correct the yields in the fourth pass. The false asymmetry associated with helicity-correlated beam properties is shown in Table 5.7. The uncertainty shown is $30 \%$ of the false asymmetry value. 

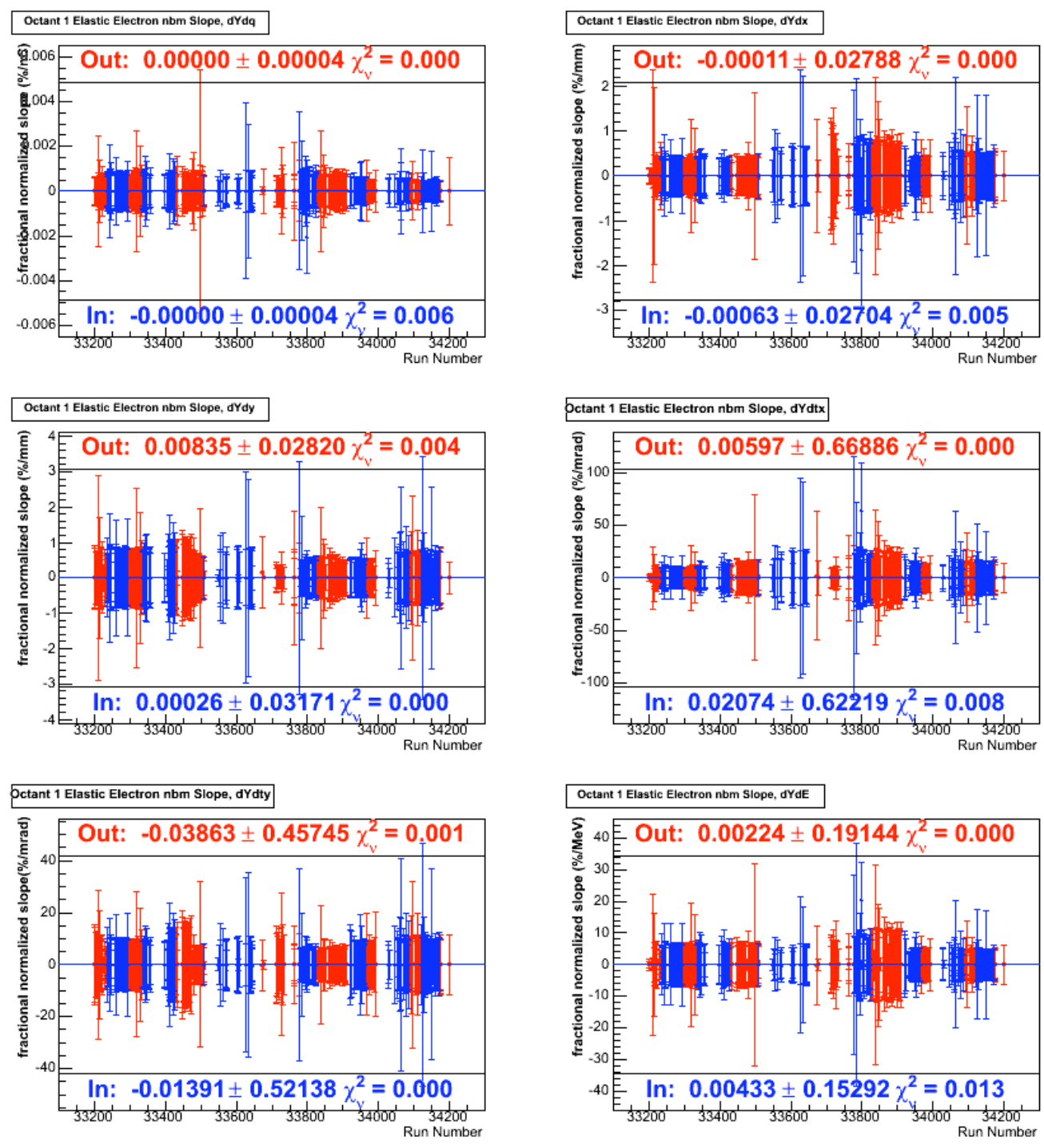

Figure 5.11: Correlation slopes, $\partial Y / \partial x_{i}$, plotted as a function of run number, where $x_{i}$ is a beam parameter for the running on a deuterium target at 362 MeV. 
Table 5.7: Calculated false asymmetries due to helicity-correlated beam parameters. The run period includes the target, beam energy, and if it does not include the entire time period, the timeframe of the data collection is also included. [Sch08]

\begin{tabular}{||c|c|c|}
\hline \hline Run period & False Asymmetry (ppm) & Uncertainty (ppm) \\
\hline \hline H 362 MeV & -0.0007 & 0.0011 \\
H 687 MeV (April 2006) & -0.0163 & 0.0136 \\
H 687 MeV (Fall 2006) & -0.0030 & 0.0022 \\
D 362 MeV & 0.0043 & 0.0024 \\
D 687 MeV (Fall 2006) & 0.0105 & 0.0120 \\
D 687 MeV (March 2007) & -0.0132 & 0.0147 \\
\hline \hline
\end{tabular}

\subsubsection{Dilution Correction}

There were five processes that contributed to the yield during the backward angle measurement. One was the process of interest, elastic (quasi-elastic) electron scattering, while the other four, inelastic electron scattering, pion contamination, $\pi^{o}$ decays, and scattering processes from the aluminum target walls, represented the background. The processes were verified for each target and beam energy combination by comparing simulation with the data. The simulations included electromagnetic radiative effects and detector efficiencies.

The percentage of each background contributing to the yield can be expressed as

$$
f_{x}=\frac{Y_{x}}{Y_{\text {total }}}
$$

where $x$ is the index of the process and $\sum_{x} f_{x}=1$. Background processes represent $10-15 \%$ of the total yield and their effect is a dilution of the true physics asymmetry. To correct for this dilution, the contribution from these processes were subtracted from the measured asymmetry. The complete analysis for this method can be found 
in [Mue09] with an overview presented here.

For each target and beam energy combination, a series of runs were taken over a range of SMS field settings as previously described in Sec. 5.1.2. Figure 5.12 shows a graphical presentation for one cell from the elastic (quasi-elastic) region, for all of the target/beam energy combinations. The nominal magnet current is shown by the dotted vertical line. For hydrogen and the low energy deuterium
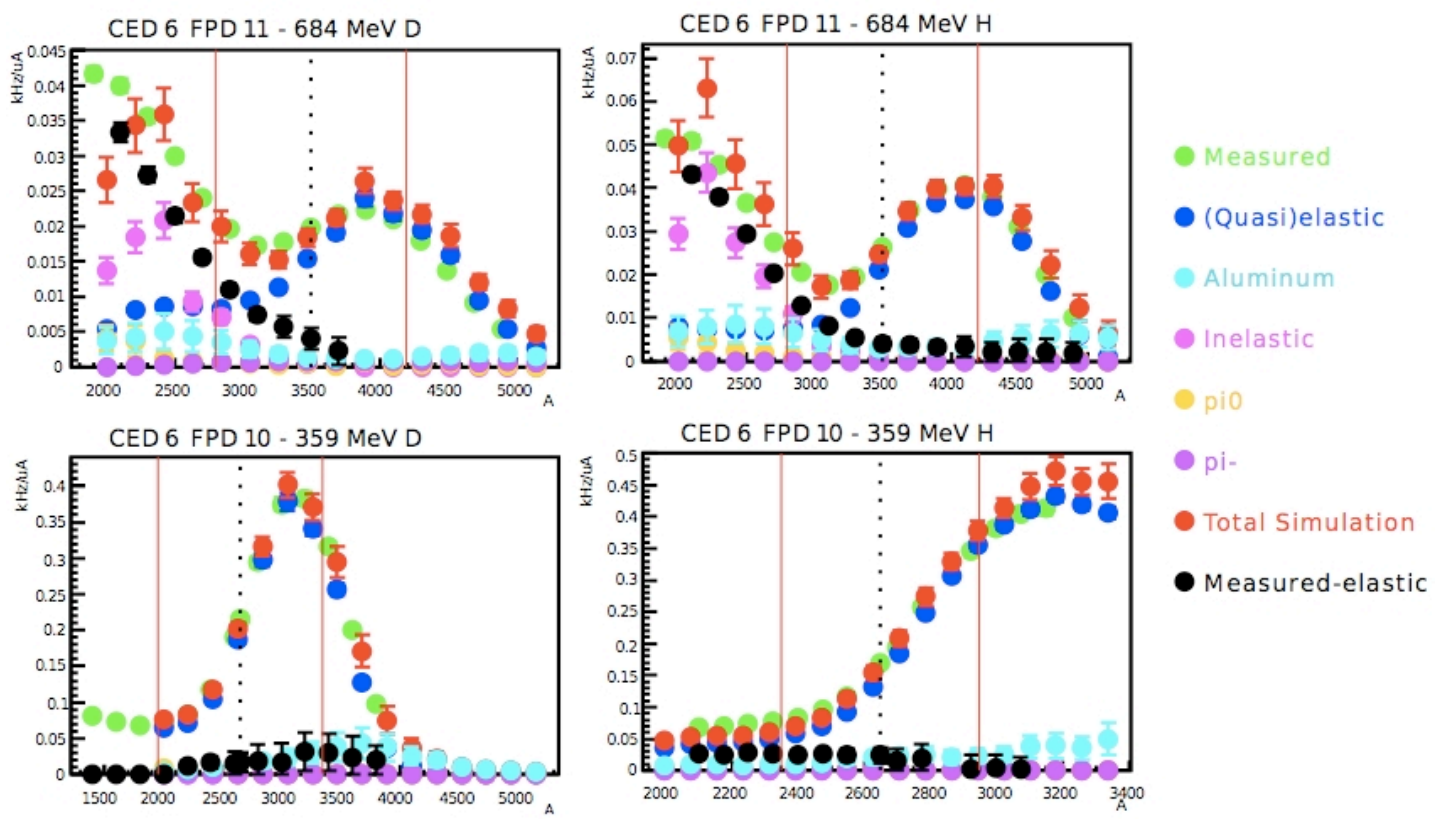

Figure 5.12: Magnet current scan for a single elastic cell on the inelastic edge of the elastic locus [Mue09]. The dotted vertical line is the nominal SMS current setting. All data points, except those labelled "measured", are from scaled Monte Carlo simulations.

data, the principal contribution to the background was from quasi-elastic electron scattering from the aluminum target walls. High energy deuterium also had a strong contribution from the $\pi^{-}$rate, which was a small effect for the other target/energy combinations. The cells selected as "elastic (quasi-elastic)" were chosen to minimize contamination from inelastic electron scattering. 
Simulations for all five processes were run using the same range of magnet current settings as the actual data. In order to effectively compare the simulations, two factors were used [Mue09]. One factor was octant dependent and scaled the simulated magnetic field, the second factor was determine through a $\chi^{2}$ minimizing technique and was applied to all of the simulation yields cell-by-cell. Using the scaled fields and scaled simulated yields, the background dilution fraction was determined for the nominal field setting as:

$$
f_{\text {back }}=1-\frac{Y_{\text {elas }}^{\text {sim }}}{Y_{\text {total }}^{\text {meas }}}
$$

where $Y_{\text {elas }}^{\text {sim }}$ is the simulated elastic (quasi-elastic) yield determined from the scaled simulated yields for the nominal field setting and $Y_{\text {total }}^{\text {meas }}$ is the total measured yield for a given cell.

The asymmetry can be expressed as a sum of the individual processes,

$$
A_{\text {meas }}=f_{\text {elas }} A_{\text {elas }}+f_{\text {inelas }} A_{\text {inelas }}+f_{a l} A_{A l}+f_{\pi^{-}} A_{\pi^{-}}+f_{\pi^{o}} A_{\pi^{o}} .
$$

This expression can be simplified by taking $A_{\pi^{o}}=A_{\pi^{-}}=0$, which is a reasonable assumption and consistent with the measured $\pi^{-}$asymmetry [Cop10] and the measured asymmetry for the background regions. Additionally, the term $f_{\text {inelas }} A_{\text {inelas }}$ can be dropped because the inelastic signal was negligible $(<0.1 \%)$ in the elastic region [Mue09]. With these assumptions, Eqn. 5.24 can be rewritten as

$$
A_{\text {elas }}=\frac{A_{\text {meas }}-f_{a l} A_{a l}-f_{\pi^{-}} A_{\pi^{-}}}{1-f_{a l}-f_{\pi^{-}}-f_{\text {other }}}
$$

where $f_{\text {other }} \approx f_{\pi^{o}}+f_{\text {inelas }}$. The measured deuterium asymmetry was used for the value of $A_{A l}$. A $5 \%$ global error was assigned to account for the fact that aluminum 
has 13 protons and 14 neutrons, rather than an equal number of each, as well as additional nuclear effects. The values of $f_{a l}$ and $f_{\text {other }}$, were determined from the magnet current scan analysis. There were data from several runs taken with only a gaseous hydrogen target. This "empty" target information was used to help model the shape of the aluminum contribution, assisting with the determination of $f_{a l}$, while an error of $100 \%$ was assigned to $f_{\text {other }}$. The values for $f_{\pi_{-}}$were determined using an analysis of analog ring sampler (ARS) data [Cop09]. Table 5.8 summarizes the average dilution values and associated error used in this calculation.

Table 5.8: The primary contributions to the elastic (quasi-elastic) electron background where $f_{\text {other }}$ represents contributions from processes not otherwise listed and consists primarily of inelastic electrons and $\pi^{\circ}$ decay [Mue09].

\begin{tabular}{||c|c|c|c|c|c||}
\hline \hline Target & Energy & $f_{a l}$ & $f_{\pi_{-}}$ & $f_{\text {other }}$ & $f_{\text {total }}$ \\
\hline \hline $\mathrm{H}$ & 362 & $0.129 \pm 0.064$ & $0 \pm 0.001$ & $0.003 \pm 0.003$ & $0.132 \pm 0.064$ \\
$\mathrm{D}$ & 362 & $0.099 \pm 0.050$ & $0 \pm 0.002$ & $0.005 \pm 0.005$ & $0.104 \pm 0.050$ \\
$\mathrm{H}$ & 687 & $0.110 \pm 0.055$ & $0 \pm 0.001$ & $0.023 \pm 0.023$ & $0.133 \pm 0.064$ \\
$\mathrm{D}$ & 687 & $0.061 \pm 0.031$ & $0.04 \pm 0.015$ & $0.029 \pm 0.029$ & $0.13 \pm 0.045$ \\
\hline \hline
\end{tabular}

Once the background corrected single cell asymmetries were determined, a weighted average was performed over the elastic locus by octant. The corrected octant asymmetries were then averaged, yielding the correction shown in Table 5.9

\subsubsection{Polarization Correction}

The correction to the measured asymmetry due to the beam polarization is

$$
A=\frac{1}{P} \times A_{\text {meas }}
$$


Table 5.9: Correction to the measured asymmetry to neutralize the contribution of the background on the physics asymmetry. The uncertainties include the associated overall-all point-to-point and global systematic uncertainties.[Mue09]

\begin{tabular}{||c|c|c||}
\hline \hline Target & $\begin{array}{c}\text { Beam Energy } \\
(\mathrm{MeV})\end{array}$ & $\Delta A_{\delta}$ correction $(\mathrm{ppm})$ \\
\hline \hline $\mathrm{H}$ & 362 & $0.5 \pm 0.11 \pm 0.40$ \\
$\mathrm{D}$ & 362 & $-0.07 \pm 0.02 \pm 0.08$ \\
$\mathrm{H}$ & 687 & $-0.1 \pm 0.61 \pm 0.86$ \\
$\mathrm{D}$ & 687 & $-2.0 \pm 0.48 \pm 0.23$ \\
\hline
\end{tabular}

where $P$ is the polarization. Details of the $G^{0}$ backward angle polarization measurements can be found in [GH08]. An overview including the final results are presented here.

Fig. 5.13 shows the Møller measurements taken during the runs with a beam energy of $687 \mathrm{MeV}$, averaged over half-wave plate states. The inner error bars are purely statistical, the outer bars are statistical and point-to-point. A constant fit to the measurements using only statistical errors, yields a value of $\quad P=85.78 \pm$ $0.07 \%$ with $\chi^{2} / \nu=4.41$. Adding the point-to-point systematic errors yields a more reasonable $\chi^{2} / \nu=0.08$ with a small change to the polarization $(P=85.81 \pm 0.16 \%)$ [GH08]. It was decided to use a single value for the beam polarization during the high energy running which was extracted from the constant fit using only the statistical errors for the fit. The final total uncertainty also includes the point-topoint systematic errors. This yields a value of [GH08]

$$
P=85.78 \pm 0.07(\text { stat }) \pm 1.38(\text { syst }) \%
$$

As mentioned in Sec. 4.9.4, the Møller Polarimeter was designed to operate at 


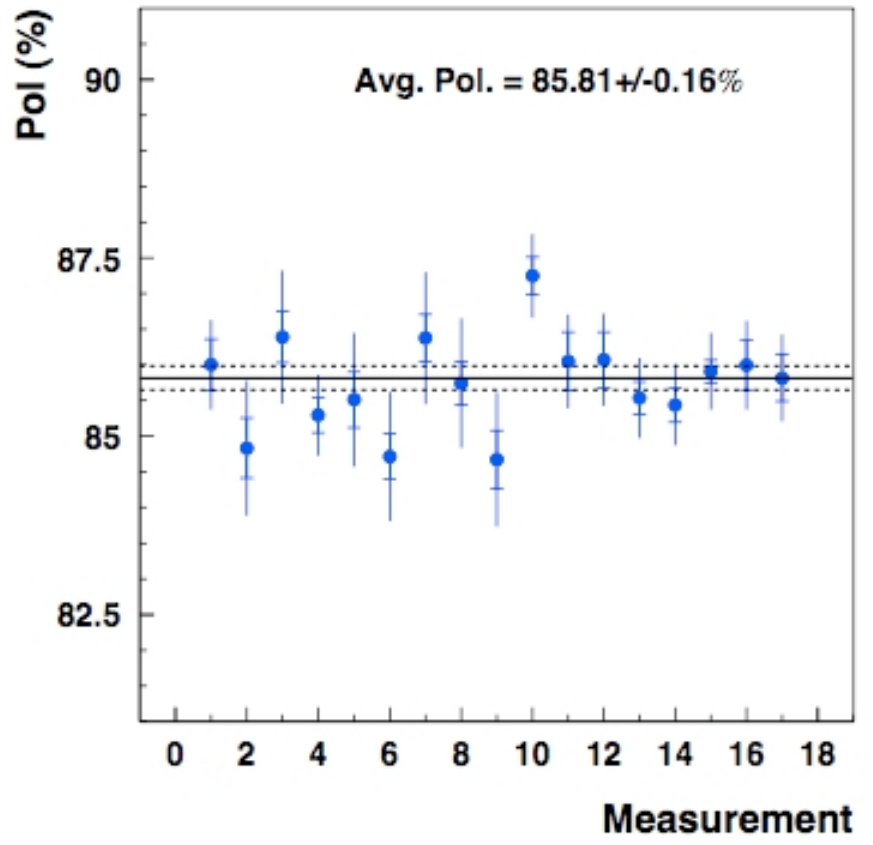

Figure 5.13: Møller polarization measurements taken during the $687 \mathrm{MeV}$ running. Inner error bars denote statistical errors only, while the outer error bars denote statistical and point-to-point added in quadrature. The solid line is a constant fit using the outer error bars, and the dashed lines are the uncertainty on the fit [GH08]. 
electron beam energies greater than $800 \mathrm{MeV}$. Several measurement attempts were made at $362 \mathrm{MeV}$, resulting in one partial measurement taken with an odd Møller optics tune at a maximum solenoid field of 1 Tesla. The result was a measured polarization of $89.22 \pm 3.24$ (stat) \pm 4.61 (syst) $\%$ [GH08].

The Mott polarimeter (see Sec. 4.17) was used to provide a consistency check with the Møller measurements during the high energy running, and was the only accurate way to measure the polarization during the low energy running. The Mott measurements are shown in Fig. 5.14. Although the polarization measurements at $687 \mathrm{MeV}$ are largely consistent, the scatter suggests some point-to-point systematic uncertainty that has not been accounted for. An additional $1.33 \%$ uncertainty was added on to each point. The extra point-to-point uncertainty $(\mathrm{dP} / \mathrm{P}=1.6 \%)$ inferred from the Mott measurements at $687 \mathrm{MeV}$, was then combined with the uncertainty of the Møller measurements to provide the total systematic uncertainty on the $362 \mathrm{MeV}$ polarization measurement. The final results of the polarization analysis are:

$$
\begin{aligned}
& \mathrm{E}_{\text {beam }}=687 \mathrm{MeV} \quad P=85.78 \pm 0.07(\text { stat }) \pm 1.38(\text { syst }) \%, \\
& \mathrm{E}_{\text {beam }}=362 \mathrm{MeV} \quad P=85.78 \pm 0.07(\text { stat }) \pm 1.95(\text { syst }) \% .
\end{aligned}
$$

Historically, the difference between the Mott and the Møller polarimetry measurements was $2 \%$ which was roughly consistent with systematic uncertainties. For the backward angle measurements, the initial analysis showed a difference of $3.5-4 \%$. It was determined that resolution of one of the Mott detectors was about $50 \%$ worse than the other detectors, which resulted in an increased sensitivity to background 


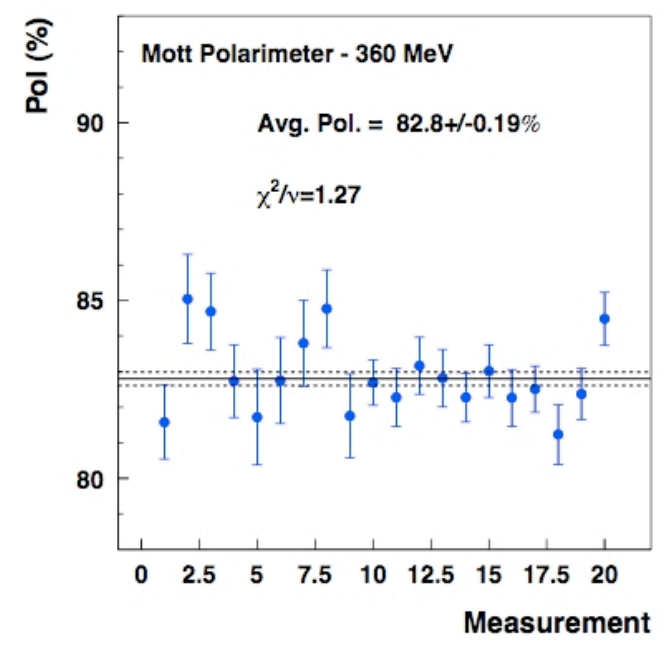

(a) Injector Mott at $362 \mathrm{MeV}$

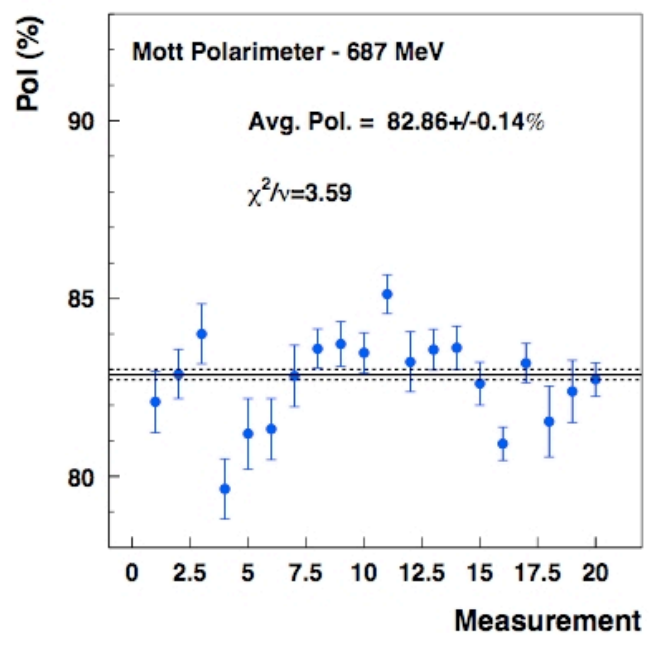

(b) Injector Mott at $687 \mathrm{MeV}$

Figure 5.14: Mott measurements during the $G^{0}$ backward angle measurement. Only statistical errors are shown.[GH08].

contributions. Studies, including a GEANT3 simulation, showed that a photon background underneath the elastic peak was not negligible [GH08]. Figure 5.15 illustrates a simulated photon background where the upper portion of the elastic peak is reasonably background free. The understanding of the deteriorated detector resolution combined with improvements in the Mott analyzing codes reduced the disagreement between the Mott and Møller measurements to 2.5\% [GH08].

\subsubsection{Transverse Polarization}

Transverse polarization components can induce false asymmetries in parityviolating electron scattering due to interference between the one-photon and twophoton exchange amplitudes. The imaginary term in the two-photon exchange amplitude creates a vector analyzing power which can cause the cross section to be 


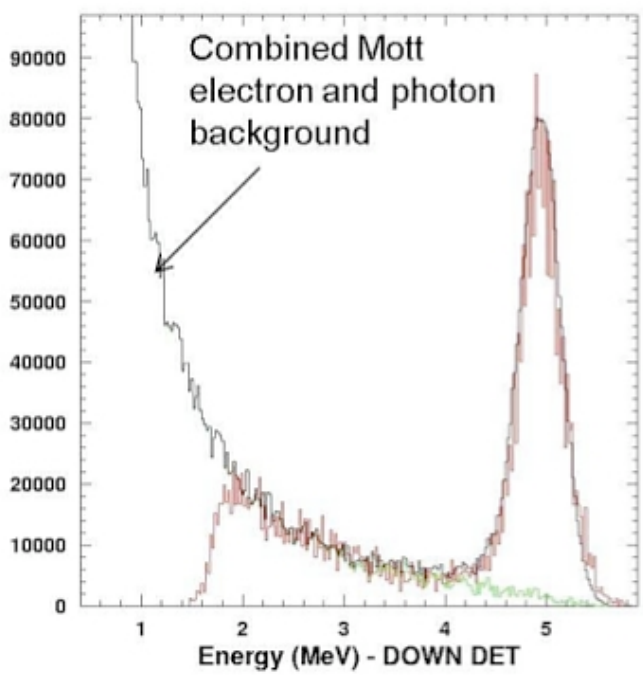

Figure 5.15: Simulated photon background assuming the beam dump was a $21 \mathrm{~mm}$ thick aluminum plate[GH08].

dependent on the polarization vector and the scattering plane.

For the $G^{0}$ backward angle measurement, the effects from residual transverse components of the beam polarization are small because of averaging over the eight detector sets placed in an azimuthally symmetric manner around the beam line. The correction was less than $1 \mathrm{ppm}$ and was not directly applied, but instead, an additional systematic uncertainty was added to the measurement. Details of the transverse polarization analysis can be found in [Mam09b]. A summary of the approach is presented here.

A transverse component of the beam polarization during longitudinal running will induce a false asymmetry, $K_{T}$ expressed as [Mam09b]

$$
K_{T}=A_{T} \frac{P_{T}}{P} A_{S}
$$

where $P_{T} / P$ is the relative transverse polarization in the longitudinal running, $A_{S}$ is the detector asymmetry, and $A_{T}$ is the amplitude of the asymmetry for a transversely 
polarized beam. As discussed in Appendix B, the LUMIs were sensitive to beam properties and measured a much higher scattering rate than the primary detectors because of their very forward position (close to the beam line, downstream from the target). $\quad$ Fig. 5.16 shows the LUMI asymmetries. Because of this

Table 5.10: Magnitudes of the measured electron asymmetry during transverse beam polarization, luminosity monitor asymmetries during both longitudinal and transverse polarization, and the magnitude of the false asymmetry, $K_{T}$, due to a residual transverse component of the beam polarization for each of the data sets [Mam10].

\begin{tabular}{||c|c|c|c|c|c||}
\hline \hline Data set & $\begin{array}{c}A_{L}^{L U M I} \\
(\mathrm{ppm})\end{array}$ & $\begin{array}{c}A_{T}^{L U M I} \\
(\mathrm{ppm})\end{array}$ & $P_{T} / P$ & $\begin{array}{c}A_{T} \\
(\mathrm{ppm})\end{array}$ & $\begin{array}{c}K_{T} \\
(\mathrm{ppm})\end{array}$ \\
\hline \hline H362 & $0.36 \pm 0.04$ & $22.5 \pm 0.8$ & 0.016 & $-176.2 \pm 9.4$ & $0.056 \pm 0.007$ \\
D362 & $0.75 \pm 0.02$ & $23.0 \pm 0.1$ & 0.033 & $-108.6 \pm 7.2$ & $0.071 \pm 0.005$ \\
H687 & $0.71 \pm 0.04$ & $19.0 \pm 0.3$ & 0.037 & $21 \pm 24$ & $0.016 \pm 0.018$ \\
D687 & $0.37 \pm 0.02$ & $18.3 \pm 0.4$ & 0.020 & $-55.2 \pm 78$ & $0.022 \pm 0.032$ \\
\hline \hline
\end{tabular}

enhanced sensitivity, the ratio $P_{T} / P$ was determined by comparing the amplitudes of sine fits for the LUMIs for longitudinal and transverse beam polarization running. The detector asymmetry, $A_{S}$, was estimated by comparing the difference in yields between the octants, which was $\pm 6 \%$. The size of the false asymmetry, $K_{T}$, is shown in Table 5.10 for all of the data sets.

\subsubsection{Electromagnetic Radiative Corrections}

Electrons scattered from proton or deuteron targets radiate real and virtual photons which result in energy loss in both the incident and scattered electrons. This affects the kinematics of the scattering event and must be accounted for. The incident electron can lose energy from target ionization or emission of bremsstrahlung 

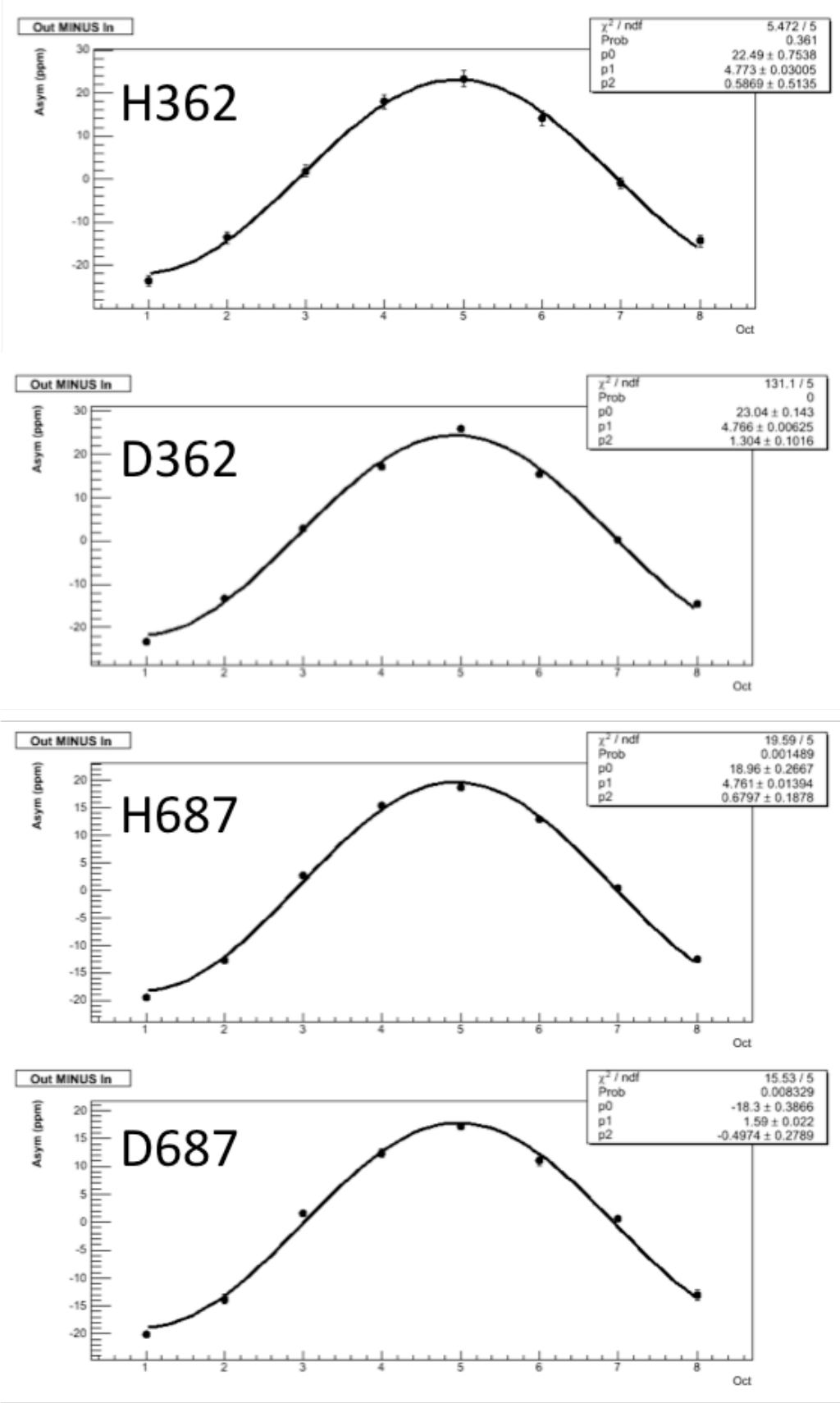

Figure 5.16: Luminosity Monitor asymmetries versus octant for each target/energy combination. Octant 1 was located at the 12 o'clock position when looking down the beam line, with the other octants placed in a clockwise manner with increasing number. The sine fits show the luminosity monitor sensitivity to any transverse component of the beam polarization. The fit parameters are p0 (amplitude), p1 (phase shift) and p2 (constant offset). 
photons. The approach taken for the electromagnetic radiative effects correction, was to calculate using simulation, the tree level asymmetry, $A_{\text {tree }}$, which is equivalent to single boson exchange and no radiative effects, and $A_{R C}$, which is the asymmetry with electromagnetic radiative effects as prescribed by Tsai [Tsa71]. The radiative correction, $R_{c}$, is simply the ratio of these two asymmetries,

$$
R_{c}=\frac{A_{\text {tree }}}{A_{R C}}
$$

Recalling that the asymmetry can be expressed as $A_{p v}=a_{o}+a_{1} G_{E}^{s}+a_{2} G_{M}^{s}+$ $a_{3} G_{A}^{e(T=1)}+a_{4} G_{A}^{s}$, the correction can be made in one of two ways. Either the calculated correction for $a_{o}$ can be applied directly to the measured asymmetry, or the measured asymmetry can be decomposed into its component parts, and a correction is applied to each of the coefficients $a_{0}$ to $a_{4}$. A comparison between the two methods was made and the result was a difference of less that $0.05 \%$. It was decided to apply the correction for $a_{0}$ to the measured physics asymmetry. These

Table 5.11: Electromagnetic radiative corrections for each $G^{0}$ backward angle measurement target and energy combination.

\begin{tabular}{||c|c|c|}
\hline \hline Target & $\begin{array}{c}\text { Beam Energy } \\
(\mathrm{MeV})\end{array}$ & correction \\
\hline \hline $\mathrm{H}$ & 362 & $1.037 \pm 0.002$ \\
$\mathrm{D}$ & 362 & $1.032 \pm 0.004$ \\
$\mathrm{H}$ & 687 & $1.037 \pm 0.002$ \\
$\mathrm{D}$ & 687 & $1.034 \pm 0.004$ \\
\hline \hline
\end{tabular}

values are summarized in Table 5.11, and are a correction on the order of $3.5 \%$. The uncertainty is dominated by the statistics used in the simulation with the deuterium values also including an additional contribution to account for uncertainty in the 
model used to extract the asymmetry. A complete discussion of the electromagnetic radiative effects calculation, including values for all of the coefficients is given in Appendix A.

\subsection{8 $Q^{2}$ Determination}

A precise determination of $Q^{2}$ was important to the backward angle measurement because the measured asymmetry is proportional to $Q^{2}$, and all of the form factors are a function of it. Because the channel of interest was elastic (quasielastic) electron scattering, $Q^{2}$ can be determined if the incident beam energy and the scattering angle are known, as discussed in the introduction to Chapter 2. The scattering angle is defined by the experimental acceptance which was determined and verified using simulation and experimental hall surveys of the target, collimator, and detector positions relative to one another [Mam09a].

The beam energy was measured, or as in the case of one run period, extracted [Mam09a] for each energy/target combination change. The final $Q^{2}$ values were obtained by running a simulation using the backward angle acceptance phase space. The simulations included electromagnetic radiative corrections, and the measured beam energy was an input variable. The results from the simulation and analysis are shown in Table 5.12 [Mam09a]. The uncertainties associated with the values

shown were determined studying the sensitivity of $Q^{2}$ to changes in values of key parameters, such as SMS current, beam position, and beam energy [Mam09a]. 
Table 5.12: $Q^{2}$ for each data set during the backward angle measurement [Mam09a].

\begin{tabular}{||c|c|c||}
\hline \hline Data Set & Beam Energy $(\mathrm{MeV})$ & $\left.Q^{2}(\mathrm{GeV} / \mathrm{c})^{2}\right)$ \\
\hline \hline H (Summer 2006) & $361.90 \pm 0.50$ & $0.2217 \pm 0.001$ \\
H (April 2006) & $685.57 \pm 0.92$ & $0.6275 \pm 0.003$ \\
H (Fall 2006) & $684.86 \pm 0.92$ & $0.6264 \pm 0.003$ \\
D (Winter 2007) & $363.05 \pm 0.66$ & $0.2193 \pm 0.001$ \\
D (Winter 2006) & $689.61 \pm 0.93$ & $0.6300 \pm 0.003$ \\
D (March 2007) & $689.42 \pm 0.93$ & $0.6299 \pm 0.003$ \\
\hline \hline
\end{tabular}

\subsection{Final Measured and Corrected Asymmetries}

This section gives the raw measured asymmetries along with a summary of all of the corrections discussed in the previous sections. The systematic errors were divided into one of two categories. Either point-to-point uncertainties that are unique to a data set, or global uncertainties which are 100\% correlated between the data sets.

Table 5.13: The raw, unblinded, measured asymmetry by target, beam energy, and $Q^{2}$. The uncertainty is purely statistical. The data have received the cut on events that are $5 \sigma$ or more away from the mean yield value (pass 2 of the replay engine), but no other corrections.

\begin{tabular}{||c|c|c|c||}
\hline \hline Target & $E_{\text {beam }}$ & $Q^{2}\left(\mathrm{GeV}^{2}\right)$ & $A_{\text {meas }}(\mathrm{ppm})$ \\
\hline \hline $\mathrm{H}$ & 362 & 0.222 & $-9.72 \pm 0.86$ \\
$\mathrm{D}$ & 362 & 0.220 & $-13.50 \pm 0.81$ \\
$\mathrm{H}$ & 687 & 0.626 & $-36.9 \pm 2.4$ \\
$\mathrm{D}$ & 687 & 0.629 & $-37.4 \pm 3.3$ \\
\hline \hline
\end{tabular}

Table 5.13 gives the unblinded values for the measured asymmetries for each of the data sets measured during the $G^{0}$ backangle experiment. The data have received the cut on events that are $5 \sigma$ or more away from the mean yield value 
(pass 2 of the replay engine), but no other corrections. It should be noted that although the unblinded values are presented here, these values were unknown to the $G^{0}$ collaborators until all of the corrections to the data describe in this chapter were determined. A summary of the corrections are presented in Tables 5.14 and 5.15.

Table 5.14: Corrections to the raw asymmetries (Table 5.13). These corrections are additive. "Other" includes corrections for helicity-correlated beam parameters, the small transverse component of beam polarization, and two-boson exchange. The uncertainties are point-to-point and global systematic [Aac $\left.{ }^{+} 10\right]$.

\begin{tabular}{||c|c|c|c|c||}
\hline \hline Target & $\begin{array}{c}Q^{2} \\
(\mathrm{GeV} / \mathrm{c})^{2}\end{array}$ & $\begin{array}{c}\text { Rate } \\
(\mathrm{ppm})\end{array}$ & $\begin{array}{c}\text { Background } \\
(\mathrm{ppm})\end{array}$ & $\begin{array}{c}\text { Other } \\
(\mathrm{ppm})\end{array}$ \\
\hline \hline $\mathrm{H}$ & 0.222 & $-0.31 \pm 0.08 \pm 0$ & $0.5 \pm 0.11 \pm 0.40$ & $0.22 \pm 0.08 \pm 0.01$ \\
$\mathrm{D}$ & 0.220 & $-0.58 \pm 0.21 \pm 0$ & $-0.07 \pm 0.02 \pm 0.08$ & $0.06 \pm 0.10 \pm 0.01$ \\
$\mathrm{H}$ & 0.626 & $-1.28 \pm 0.18 \pm 0$ & $-0.1 \pm 0.61 \pm 0.86$ & $0.29 \pm 0.11 \pm 0.01$ \\
$\mathrm{D}$ & 0.629 & $-7.0 \pm 1.8 \pm 0$ & $-2.0 \pm 0.48 \pm 0.23$ & $0.34 \pm 0.21 \pm 0.01$ \\
\hline \hline
\end{tabular}

Table 5.15: Corrections to the raw asymmetries (Table 5.13). These corrections are multiplicative. They include the beam polarization $(1 / P)$ and the correction for electromagnetic (EM) radiative effects. The uncertainties are point-to-point and global systematic $\left[\mathrm{Aac}^{+} 10\right]$.

\begin{tabular}{||c|c|c|c||}
\hline \hline Target & $Q^{2}(\mathrm{GeV} / \mathrm{c})^{2}$ & Polarization & EM radiative \\
\hline \hline $\mathrm{H}$ & 0.222 & $(1 / 0.858) \pm 0.02 \pm 0.01$ & $1.037 \pm 0.002 \pm 0$ \\
$\mathrm{D}$ & 0.220 & $(1 / 0.858) \pm 0.02 \pm 0.01$ & $1.032 \pm 0.004 \pm 0$ \\
$\mathrm{H}$ & 0.626 & $(1 / 0.858) \pm 0.02 \pm 0.01$ & $1.037 \pm 0.002 \pm 0$ \\
$\mathrm{D}$ & 0.629 & $(1 / 0.858) \pm 0.02 \pm 0.01$ & $1.034 \pm 0.004 \pm 0$ \\
\hline \hline
\end{tabular}

After applying the corrections in Tables 5.14 and 5.15 to the values in Table 5.13, the final, corrected physics asymmetries, $A_{\text {phys }}$, are obtained. These values are shown in Table 5.16 .

The next chapter discusses how the vector strange and axial form factors are 
Table 5.16: The final physics asymmetries from the $G^{0}$ backward angle measurement. The uncertainties are statistical, point-to-point, and global systematic [Aac $\left.{ }^{+} 10\right]$.

\begin{tabular}{||c|c|c||}
\hline \hline Target & $Q^{2}(\mathrm{GeV} / \mathrm{c})^{2}$ & $A_{\text {phys }}(\mathrm{ppm})$ \\
\hline \hline $\mathrm{H}$ & 0.222 & $-11.25 \pm 0.86 \pm 0.27 \pm 0.43$ \\
$\mathrm{D}$ & 0.220 & $-16.93 \pm 0.81 \pm 0.41 \pm 0.21$ \\
$\mathrm{H}$ & 0.626 & $-45.9 \pm 2.4 \pm 0.8 \pm 1.0$ \\
$\mathrm{D}$ & 0.629 & $-55.5 \pm 3.3 \pm 2.0 \pm 0.7$ \\
\hline \hline
\end{tabular}

extracted from $A_{\text {phys }}$. 


\section{Chapter 6}

\section{Results and Conclusions}

The asymmetries measured and reported in Chapter 5 are combined with the measured asymmetries from the $G^{0}$ forward angle measurement $\left[\mathrm{A}^{+} 05\right]$ to extract the strange vector form factors, as described in Chapter 2. The key steps in this process are determining the measured asymmetries for a common four-momentum transfer for both the backward and forward angle measurements, selecting the parameterization of the electromagnetic form factors for both the proton and neutron, and calculating the asymmetry coefficients. The three asymmetry measurements (hydrogen forward angle, hydrogen backward angle, and deuterium backward angle) are then used in a system of three equations with three unknowns, $G_{E}^{s}, G_{M}^{s}$, and $G_{A}^{e}$. The following sections explain each step in detail.

\subsection{Combining $G^{0}$ Forward and $G^{0}$ Backward Angle Measurements}

In order to combine the measurements made on the various targets at the different kinematics, it is necessary to extract a measured value at a common $Q^{2}$. The backward angle measurements introduced in Chapter 5 are repeated here, in Table 6.1, for convenience. These asymmetry values were adjusted to common $Q^{2}$ values of $0.221(\mathrm{GeV} / \mathrm{c})^{2}$ and $0.628(\mathrm{GeV} / \mathrm{c})^{2}$ using the sensitivity of the asymmetry to the four-momentum transfer, $\delta A / \delta Q^{2}$. The values used were $\delta A / \delta Q^{2}=-123.0 \mathrm{ppm} /$ 
$(\mathrm{GeV} / \mathrm{c})^{2}$ for the high energy hydrogen and $\delta A / \delta Q^{2}=-113.8 \mathrm{ppm} /(\mathrm{GeV} / \mathrm{c})^{2}$ for the low energy hydrogen measurement. For the deuterium target, the values used Table 6.1: The final physics asymmetries from the $G^{0}$ backward angle measurement. The uncertainties are statistical, point-to-point, and global systematic [Aac $\left.{ }^{+} 10\right]$.

\begin{tabular}{c|c|c}
\hline \hline Target & $Q^{2}(\mathrm{GeV} / \mathrm{c})^{2}$ & $A_{\text {phys }}(\mathrm{ppm})$ \\
\hline \hline $\mathrm{H}$ & 0.222 & $-11.25 \pm 0.86 \pm 0.27 \pm 0.43$ \\
$\mathrm{D}$ & 0.220 & $-16.93 \pm 0.81 \pm 0.42 \pm 0.21$ \\
$\mathrm{H}$ & 0.626 & $-45.87 \pm 2.41 \pm 0.82 \pm 1.03$ \\
$\mathrm{D}$ & 0.629 & $-55.55 \pm 3.31 \pm 1.96 \pm 0.71$ \\
\hline \hline
\end{tabular}

were $\delta A / \delta Q^{2}=-138.6 \mathrm{ppm} /(\mathrm{GeV} / \mathrm{c})^{2}$ and $\delta A / \delta Q^{2}=-111.2 \mathrm{ppm} /(\mathrm{GeV} / \mathrm{c})^{2}$, for the high and the low energy measurements, respectively. The asymmetry values, adjusted to the common $Q^{2}$ values, are shown in Table 6.2.

Table 6.2: The final physics asymmetries from the $G^{0}$ backward angle measurement adjusted to the common $Q^{2}$ values. The uncertainties are statistical, point-to-point, and global systematic $\left[\mathrm{Aac}^{+} 10\right]$.

\begin{tabular}{c|c|c}
\hline \hline Target & $Q^{2}(\mathrm{GeV} / \mathrm{c})^{2}$ & $A_{\text {phys }}(\mathrm{ppm})$ \\
\hline \hline $\mathrm{H}$ & 0.221 & $-11.10 \pm 0.86 \pm 0.27 \pm 0.43$ \\
$\mathrm{D}$ & 0.221 & $-17.07 \pm 0.81 \pm 0.42 \pm 0.21$ \\
$\mathrm{H}$ & 0.628 & $-46.05 \pm 2.41 \pm 0.82 \pm 1.03$ \\
$\mathrm{D}$ & 0.628 & $-55.38 \pm 3.31 \pm 1.97 \pm 0.71$ \\
\hline \hline
\end{tabular}

Figure 6.1 shows physics asymmetries measured during the forward angle portion of the $G^{0}$ experiment using a hydrogen target. The inner error bar is the statistical uncertainty, and the outer error bar is the statistical and the point-topoint systematic uncertainties combined in quadrature for each data point. The global uncertainty is shown at the top of the graph in gray. $A_{N V S}$, the asymmetry 


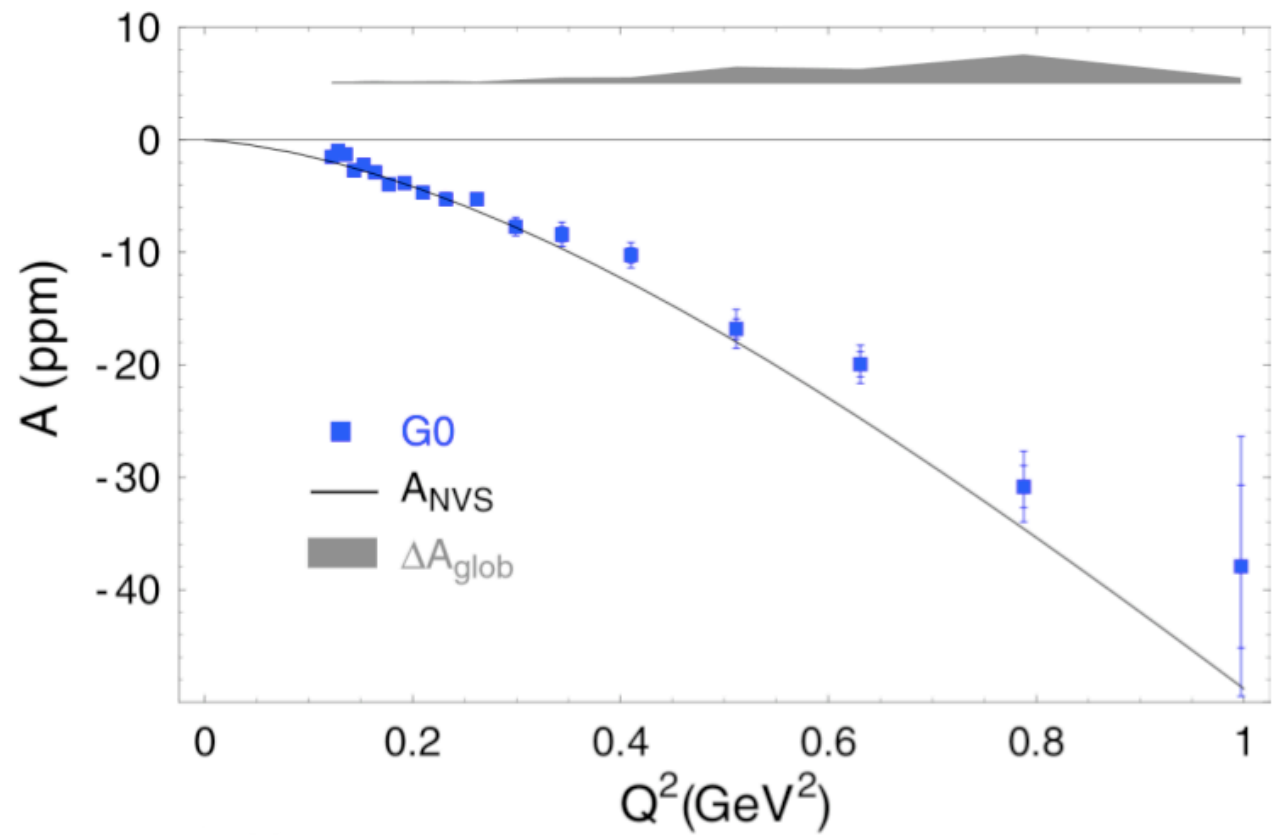

Figure 6.1: The physics asymmetries measured during the forward angle portion of the $G^{0}$ experiment which used a hydrogen target. The inner error bars are the statistical uncertainty, and the outer error bars are the statistical and point-to-point systematic uncertainties combined in quadrature. The global uncertainty, $\Delta A_{\text {glob }}$, is shown at the top of the graph in gray. $A_{N V S}$, is shown as the solid gray line. 
calculated when $G_{E}^{s}=G_{M}^{s}=0$, is shown on the graph as the solid gray line. The form factors used to compute $A_{N V S}$ were from a fit by Kelly [Kel04]. The difference between the measured points and $A_{N V S}$ gives a feel for the contribution of the strange quarks to the asymmetry.

To extract the measured forward angle asymmetries at the common $Q^{2}$ values, a simple interpolation method was used, resulting in the values shown in Table 6.3. The uncertainties are statistical, point-to-point systematic, and global systematic respectively (the same as for the backward angle measurement).

Table 6.3: The $G^{0}$ forward angle physics asymmetries interpolated to $Q^{2}$ values of the backward angle measurement.

\begin{tabular}{c|c|c}
\hline \hline Target & $Q^{2}(\mathrm{GeV} / \mathrm{c})^{2}$ & $A_{\text {phys }}(\mathrm{ppm})$ \\
\hline \hline $\mathrm{H}$ & 0.221 & $-4.77 \pm 0.36 \pm 0.21 \pm 0.23$ \\
$\mathrm{H}$ & 0.628 & $-20.71 \pm 0.78 \pm 0.90 \pm 1.31$ \\
\hline \hline
\end{tabular}

\subsection{Electromagnetic Form Factor Parameterization}

Kelly's [Kel04] parameterization of the electromagnetic form factors were used for the extraction of the strange vector and axial-vector form factors, as well as for the calculation of the deuterium asymmetry coefficients discussed in the next section. Kelly's data selection emphasized recoil or target polarization data and for $G_{E}^{p}$, he omitted data using the Rosenbluth method for $Q^{2}>1(\mathrm{GeV} / \mathrm{c})^{2}$. He used 
functions of $Q^{2}$ to parameterize the nucleon electromagnetic form factors [Kel04],

$$
G\left(Q^{2}\right) \propto \frac{\sum_{k=0}^{n} a_{k} \tau^{k}}{1+\sum_{k=1}^{n+2} b_{k} \tau^{k}},
$$

for $G_{E}^{p}, G_{M}^{p}$, and $G_{E}^{n}$.
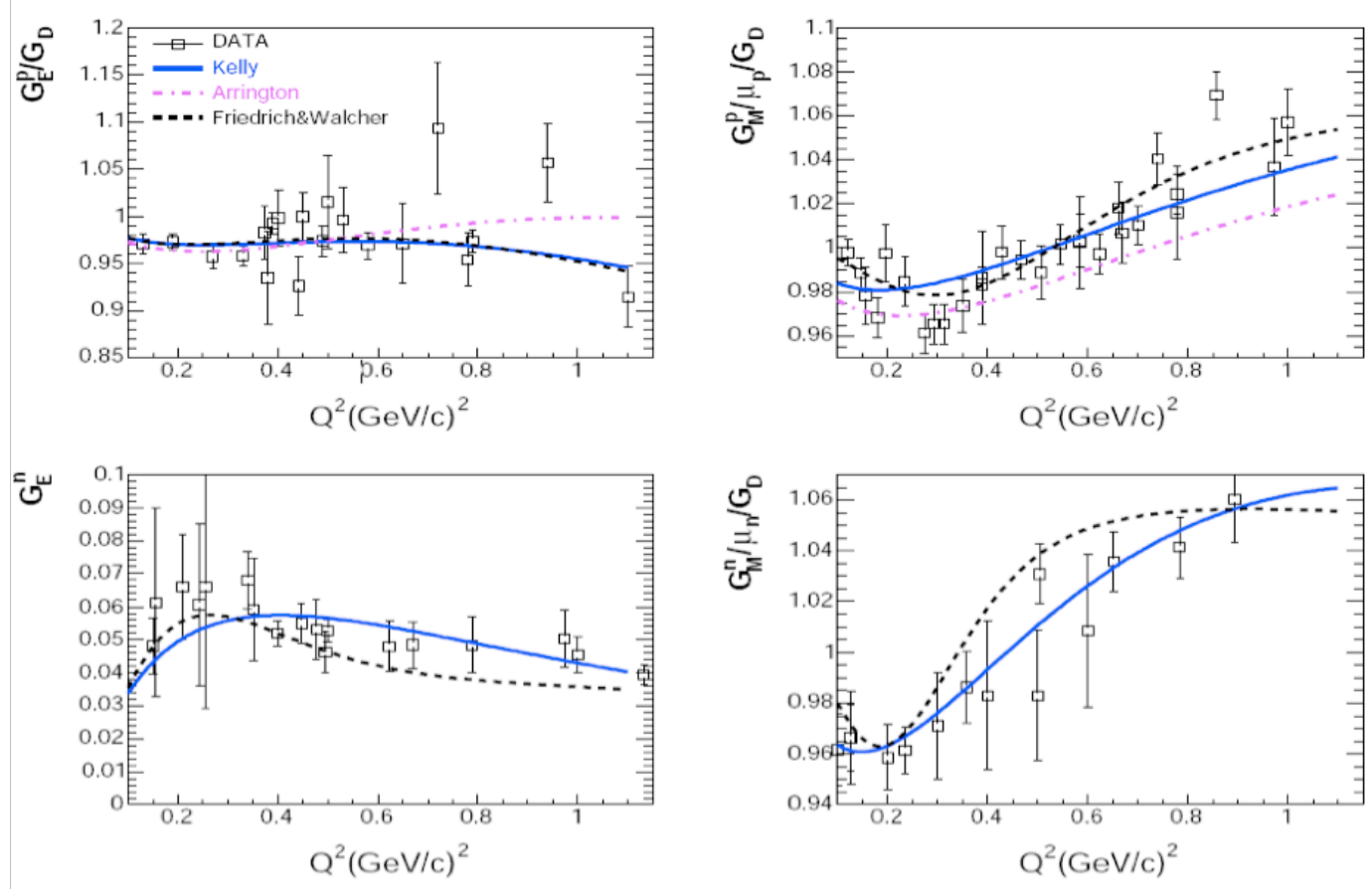

Figure 6.2: Fits for the electromagnetic form factors of the neutron and proton with a range of $Q^{2}$ from 0.1 to $1.1(G e v / c)^{2} . G_{E}^{p}, G_{M}^{p}$, and $G_{E}^{n}$ were normalized using their corresponding dipole fits. The data and the fit for Kelly (solid blue) are from [Kel04]. The other curves in the figures represent different form factor parameterizations: (pink dot-dashed $=$ [Arr04], black dashed $=$ Friedrich-Walcher [FW03].) The Arrington parameterization is for the proton only. This figure is taken from [Liu06].

The Bates Large Acceptance Spectrometer Toroid (BLAST) collaboration recently published results of measurements of the nucleon form factors by means of scattering of polarized electrons from vector-polarized hydrogen and deuterium 
$\left[\mathrm{C}^{+} 07\right]$. Their results are shown in Fig. 6.3 and are in good agreement with the Kelly parameterization, which is also shown.

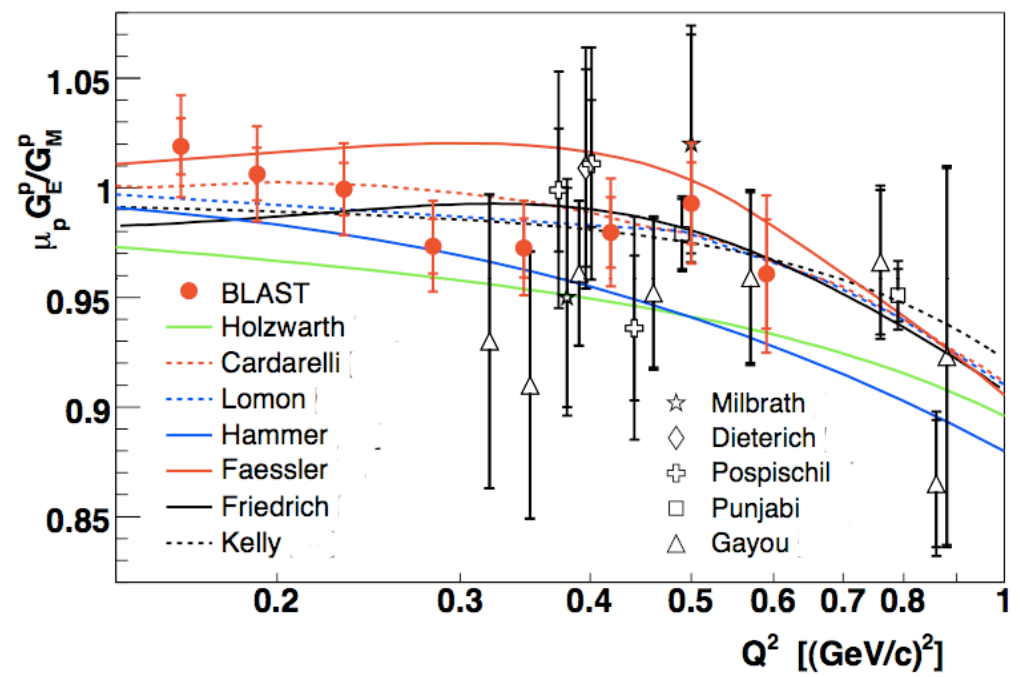

Figure 6.3: Results of $\mu G_{E}^{p} / G_{M}^{p}$ shown with the world polarized data and several models, including the Kelly parameterization [ $\left.C^{+} 07\right]$.

Because of a lack of data for $G_{E}^{n}$, Kelly used a modified Galster parameterization $\left[\mathrm{G}^{+} 71\right]$

$$
G_{E}^{n}\left(Q^{2}\right)=\frac{A_{\tau}}{1+B_{\tau}} G_{D}\left(Q^{2}\right)
$$

where $G_{D}=\left(1+Q^{2} / \Lambda^{2}\right)^{-2}$ is the dipole form factor and $\Lambda^{2}=0.71(\mathrm{GeV} / \mathrm{c})^{2}$.

Other form factor parameterizations include one by Friedrich and Walcher, who used a data set similar to Kelly's [FW03]. For the proton, Arrington also published a fit using cross section data [Arr04]. All three of these fits are shown in Fig. 6.2. The Kelly form factors were selected because the straight-forward parameterization lacked many of the physics assumptions found in some of the models such as Friedrich and Walcher, and because the Kelly form factors were used in both the deuterium coefficient calculation provided by Schiavilla [Sch06] 
(discussed in the next section), and the published forward angle results $\left[\mathrm{A}^{+} 05\right]$.

\subsection{Asymmetry Coefficients}

Each asymmetry can be expressed as,

$$
A=a 0^{\prime}+a 1 G_{E}^{s}+a 2 G_{M}^{s}+a 3\left(1+R_{A}^{T=1}\right) G_{a}^{e(T=1)}
$$

where $a 0^{\prime}=a 0+a 4 G_{A}^{s}$. Recall that

$$
G_{A}^{e}=-\left(1+R_{A}^{T=1}\right) G_{A}^{T=1}+\sqrt{3} R_{A}^{T=0} G_{A}^{T=0}+\left(1+R_{A}^{(0)}\right) G_{A}^{s} .
$$

The "a0" term in Eqn. 6.3 includes the non-strange isoscalar portion of the axial form factor. The axial form factor $G_{A}^{e}$, Eqn. 6.4, was constructed after the value $\left(1+R_{A}^{T=1}\right) G_{a}^{e(T=1)}$ was determined. Recall from the discussion in Sec. 2.2.2, that the higher order terms associated with $R_{A}^{T=1}$ were expected to be large. Measuring and constraining $\left(1+R_{A}^{T=1}\right) G_{a}^{e(T=1)}$ significantly reduces the uncertainty associated with $G_{A}^{e}$.

Two different techniques were used to calculate the coefficients, depending upon the target. For hydrogen, we can combine Eqn. 6.3 with Eqn. 2.63 and

Table 6.4: Radiative correction factors, including the baryon beta decay parameters $(F$ and $D)$ and $G_{A}^{s}$.

\begin{tabular}{|c|c||c|c|}
\hline \hline$R$ Factor & Value & $R$ Factor & Value \\
\hline \hline$R_{V}^{p}$ & -0.054568 & $R_{V}^{n}$ & -0.011679 \\
$R_{V}^{(0)}$ & -0.011789 & $R_{A}^{T=1}$ & -0.259163 \\
$R_{A}^{T=0}$ & $-0.23826 \pm 0.20$ & $R_{A}^{(0)}$ & $-0.5517526 \pm 0.0 .55$ \\
$G_{A}^{s}$ & $-0.08 \pm 0.04$ & $3 F-D$ & $0.585 \pm 0.023$ \\
\hline \hline
\end{tabular}


Eqn. 2.69 to express the coefficients in the following manner:

$$
\begin{aligned}
a 0 & +a 4 G_{A}^{s}=-\left(\frac{G_{F} Q^{2}}{4 \pi \alpha \sqrt{2}}\right) \frac{1}{\left[\epsilon\left(G_{E}^{\gamma, p}\right)^{2}+\tau\left(G_{M}^{\gamma, p}\right)^{2}\right]} \\
& \times\left[\left(\epsilon\left(G_{E}^{\gamma, p}\right)^{2}+\tau\left(G_{M}^{\gamma, p}\right)^{2}\right)\left(1-4 \sin ^{2} \theta_{w}\right)\left(1+R_{V}^{p}\right)\right. \\
& -\left(\epsilon G_{E}^{\gamma, p} G_{E}^{\gamma, n}+\tau G_{M}^{\gamma, p} G_{M}^{\gamma, n}\right)\left(1+R_{V}^{n}\right) \\
& \left.-\epsilon^{\prime}\left(1-4 \sin ^{2} \theta_{w}\right) G_{M}^{\gamma, p}\left(\sqrt{3} R_{A}^{T=0} G_{A}^{T=0}+\left(1+R_{A}^{0}\right) G_{A}^{s}\right)\right] \\
a 1 & =\left(\frac{G_{F} Q^{2}}{4 \pi \alpha \sqrt{2}}\right) \frac{1}{\left[\epsilon\left(G_{E}^{\gamma, p}\right)^{2}+\tau\left(G_{M}^{\gamma, p}\right)^{2}\right]}\left(\epsilon G_{E}^{p}\left(1+R_{V}^{0}\right)\right) \\
a 2 & =\left(\frac{G_{F} Q^{2}}{4 \pi \alpha \sqrt{2}}\right) \frac{1}{\left[\tau\left(G_{E}^{\gamma, p}\right)^{2}+\tau\left(G_{M}^{\gamma, p}\right)^{2}\right]}\left(\tau G_{M}^{p}\left(1+R_{V}^{0}\right)\right) \\
a 3 & =-\left(\frac{G_{F} Q^{2}}{4 \pi \alpha \sqrt{2}}\right) \frac{1}{\left[\epsilon\left(G_{E}^{\gamma, p}\right)^{2}+\tau\left(G_{M}^{\gamma, p}\right)^{2}\right]}\left(\epsilon^{\prime}\left(1-4 \sin ^{2} \theta_{w}\right) G_{M}^{p}\right)
\end{aligned}
$$

The values used for $G_{A}^{s}$ and $G_{A}^{T=0}$ are discussed in Sec. 2.3. The electroweak radiative correction $R$ factors used to extract the form factors are shown in Table 6.4. Standard parameter values, either obtained from the Particle Data Book [AoPDG08], or from other sources as discussed in Chapter 2, are shown in Table 6.5.

Calculation of the deuterium coefficients is not as straightforward as it is for hydrogen. The quasi-elastic deuterium asymmetry is dependent upon both the momentum and the energy transfer and is modified by nucleon-nucleon interaction effects. The coefficients were determined using a calculation provided by R. Schiavilla [Sch06, SCP04] in which he used the AV18 nucleon-nucleon interaction model as described in [DSvK01]. The calculation included lowest-order relativistic corrections to the one-body electromagnetic and weak currents.

Because the calculation was computationally intensive, an analytic form was 
Table 6.5: The standard parameters used in the form factor extraction calculation. The uncertainties are either negligible or as in the case of $\Lambda$, included in the overall form factor uncertainties.

\begin{tabular}{|c|c|}
\hline \hline$R$ Factor & Value \\
\hline \hline$G_{F}$ & $1.16639 \times 10^{-5}$ \\
$\alpha$ & $1 / 137.036$ \\
$\sin ^{2} \theta_{W}$ & 0.2312 \\
$M_{p}$ & $0.938272 \mathrm{GeV}$ \\
$\mu_{p}$ & 2.79285 \\
$\mu_{n}$ & -1.91304 \\
$\Lambda^{2}$ & $0.711 \mathrm{GeV}^{2}$ \\
$\Lambda_{A}^{2}$ & $1.0 \mathrm{GeV}^{2}$ \\
$g_{A} / g_{V}$ & 1.2695 \\
\hline \hline
\end{tabular}

not available and instead, the calculation was performed at the National Energy Research Scientific Computing Center (NERSC) at Lawrence Berkeley National Laboratory (LBNL) for selected kinematics which covered the range of the $G^{0}$ backward angle acceptance. The results of the NERSC calculations were data tables that provided the electromagnetic and neutral weak response functions at the selected kinematics. A Monte Carlo simulation (GEANT) incorporating the look-up tables and employing an interpolation scheme was used to calculate the quasi-elastic asymmetry and cross section over the entire backward angle acceptance. A description of this technique is in Sec. A.4. An overview of the procedure to calculate the deuterium asymmetry coefficients using R. Schiavilla's calculation is presented below.

To determine the deuterium asymmetry coefficients, it's best to express the asymmetry as a ratio of parity-conserving (EM) and parity-violating transverse and 
longitudinal response functions [BPS05].

$$
A_{d}=\frac{G_{F} Q^{2}}{4 \pi \alpha \sqrt{2}} \frac{W^{P V}}{W^{E M}}
$$

where

$$
\begin{aligned}
W^{E M} & =v_{L} R_{L}^{\gamma}(q, \omega)+v_{T} R_{T}^{\gamma}(q, \omega) \\
W^{P V} & =v_{L} R_{L}^{Z}(q, \omega)+v_{T} R_{T}^{Z}(q, \omega)+v_{T^{\prime}} R_{T^{\prime}}^{A}(q, \omega)
\end{aligned}
$$

and $(\omega, \vec{q})$ is the momentum four-vector of the virtual photon. The electron kinematic factors $v_{L}, v_{T}$, and $v_{T^{\prime}}$ are

$v_{L}=\left[\frac{Q^{2}}{q^{2}}\right]^{2}, v_{T}=\frac{1}{2}\left[\frac{Q^{2}}{q^{2}}\right]+\tan ^{2}\left(\frac{\theta}{2}\right)$, and $v_{T^{\prime}}=\tan \left(\frac{\theta}{2}\right)\left[\left|\frac{Q^{2}}{q^{2}}\right|+\tan ^{2}\left(\frac{\theta}{2}\right)\right]^{1 / 2}$

and $R_{L}$ and $R_{T}$ are the nuclear electroweak response functions, which depend on $\mathbf{q}$ and $\omega$. The $Z$ and $A$ subscripts refer to hadronic vector and axial-vector response functions, respectively. The response functions can be expressed as [SCP04]

$$
\begin{aligned}
R_{L}^{\gamma, a}(q, \omega) & =\overline{\sum_{i}} \sum_{f} \delta\left(\omega+E_{d}-E_{f}\right) \operatorname{Re}\left[\rho_{f i}^{\gamma}(\vec{q})\left[\rho_{f i}^{a *}(\vec{q})\right]\right. \\
R_{T}^{\gamma, a}(q, \omega) & =\overline{\sum_{i}} \sum_{f} \delta\left(\omega+E_{d}-E_{f}\right) \operatorname{Re}\left[\mathbf{j}_{\mathbf{f i}}^{\gamma}(\vec{q}) \mathbf{j}_{\mathbf{f}}^{\mathbf{a} *}(\vec{q})\right] \\
R_{T^{\prime}}^{\gamma, 5}(q, \omega) & =\overline{\sum_{i}} \sum_{f} \delta\left(\omega+E_{d}-E_{f}\right) \operatorname{Re}\left[\mathbf{j}_{\mathbf{f i}}^{\gamma}(\vec{q}) \times \mathbf{j}_{\mathbf{f i}}^{5 *}(\vec{q})\right]
\end{aligned}
$$

where $E_{d}$ is the ground-state energy of the deuteron, $E_{f}$ is the energy of the final scattering state, the superscript $a$ can be either a $\gamma$ (for the two electromagnetic response functions), or $Z$ (for neutral weak vector response functions), and 5 denotes an axial current. 
The parity-violating response functions which are bilinear in the charges and currents, are further decomposed in the calculation by R. Schiavilla with the strange quark pieces separated out as follows [Bei08]:

$$
\begin{aligned}
& R_{L}^{\gamma, Z}=R_{L}^{V}+R_{L}^{s} \\
& R_{T}^{\gamma, Z}=R_{T}^{V}+R_{T}^{c s}+R_{T}^{s} \\
& R_{A}^{\gamma, 5}=\left(R_{L T}^{A}\right)^{00}+\left(R_{L T}^{A}\right)^{01}+\left(R_{L T}^{A}\right)^{10}+\left(R_{L T}^{A}\right)^{11}
\end{aligned}
$$

The indices on the axial response functions correspond to isospin of the axial and electromagnetic currents, respectively. Note that the transverse response function has a small contribution, $R_{T}^{c s}$, resulting from a convection current that contributes to the charge and is thus proportional to $G_{E}^{s}$ [Bei08]. Asymmetry terms for deuterium can be expressed as [Bei08]:

$$
\begin{aligned}
& A_{0}=-\left(\frac{G_{F} Q^{2}}{2 \pi \alpha \sqrt{2}}\right) \frac{v_{L} R_{L}^{V}+v_{T} R_{T}^{V}}{v_{L} R_{L}^{\gamma}+v_{T} R_{T}^{\gamma}}, \\
& A_{1}=-\left(\frac{G_{F} Q^{2}}{2 \pi \alpha \sqrt{2}}\right) \frac{v_{L} R_{L}^{s}+v_{T} R_{T}^{c s}}{v_{L} R_{L}^{\gamma}+v_{T} R_{T}^{\gamma}} \\
& A_{2}=-\left(\frac{G_{F} Q^{2}}{2 \pi \alpha \sqrt{2}}\right) \frac{v_{T} R_{T}^{s}}{v_{L} R_{L}^{\gamma}+v_{T} R_{T}^{\gamma}}, \\
& A_{3}=-\left(\frac{G_{F} Q^{2}}{2 \pi \alpha \sqrt{2}}\right) \frac{\left(1-4 \sin ^{2} \theta_{w}\right) v_{T^{\prime}}\left[\left(R_{L T}^{A}\right)^{10}+\left(R_{L T}^{A}\right)^{11}\right.}{v_{L} R_{L}^{\gamma}+v_{T} R_{T}^{\gamma}}, \\
& A_{4}=-\left(\frac{G_{F} Q^{2}}{2 \pi \alpha \sqrt{2}}\right) \frac{\left(1-4 \sin ^{2} \theta_{w}\right) v_{T^{\prime}}\left[\left(R_{L T}^{A}\right)^{00}+\left(R_{L T}^{A}\right)^{01}\right.}{v_{L} R_{L}^{\gamma}+v_{T} R_{T}^{\gamma}} .
\end{aligned}
$$

The coefficients in Eqn. 6.3 are then [Bei08]:

$$
\begin{array}{rlrl}
a 0 & =A_{0}+a 4 \frac{R_{A}^{8}}{R_{A}+G_{A}^{s}} & a 4=\frac{A_{4}}{R_{A}+G_{A}^{s}} \\
a 1=\frac{A_{1}}{G_{E}^{s}} \quad a 2=\frac{A_{2}}{G_{M}^{s}} & a 3=\frac{A_{3}}{G_{A}^{e(T=1)}} .
\end{array}
$$

The form of the equations above, allow the removal of the parameterizations of the strange and axial vector form factors used by Schiavilla in his calculations. The 
parameter values used in Eqn. 6.15 were:

$$
\begin{aligned}
G_{M}^{s}\left(Q^{2}\right) & =\frac{G_{M}^{s}(0)}{\left(1+Q^{2} / M_{V}^{2}\right)^{2}}, \\
G_{E}^{s}\left(Q^{2}\right) & =\frac{\tau G_{E}^{s}(0)}{\left(1+Q^{2} / \Lambda^{2}\right)^{2}\left(1+\lambda_{s} \tau\right)} \\
G_{A}^{e(T=1)}\left(Q^{2}\right) & =-\frac{g_{A}}{\left(1+Q^{2} / M_{A 1}^{2}\right)^{2}}, \\
G_{A}^{s}\left(Q^{2}\right) & =-\frac{G_{A}^{s}(0)}{\left(1+Q^{2} / M_{A 0}^{2}\right)^{2}}, \\
R_{A}^{8} & =-\frac{R_{A}^{0} G_{A}^{8}(0)}{\left(1+Q^{2} / M_{A 0}^{2}\right)^{2}} .
\end{aligned}
$$

It should be emphasized that the final asymmetry coefficients and measurements are completely independent of the values shown in Eqn. 6.16 and their input parameters shown in Table 6.6.

Table 6.6: Values of the parameters used in the calculation of the deuterium asymmetry coefficients [Bei08].

\begin{tabular}{|c|c||c|c|}
\hline \hline Parameter & Value & Parameter & Value \\
\hline \hline$G_{M}^{s}(0)$ & -0.3 & $M_{V}$ & $0.84221 \mathrm{GeV}$ \\
$G_{E}^{s}(0)$ & -2.0 & $\lambda_{s}$ & 5.6 \\
$G_{A}^{8}(0)$ & 0.3554 & $M_{A 1}$ & $1.068 \mathrm{GeV}$ \\
$g_{A}$ & 1.2695 & $M_{A 0}$ & $1.00 \mathrm{GeV}$ \\
$R_{V}^{(T=0)}$ & -0.0091121 & $R_{V}^{(T=1)}$ & -0.0140914 \\
$\Lambda^{2}$ & $0.711(\mathrm{GeV})^{2}$ & & \\
\hline \hline
\end{tabular}

The measured asymmetry values and the calculated asymmetry coefficients used to extract the vector strange and axial form factors are given in Table 6.7 for both the forward angle measurement on a hydrogen target, and the backward angle measurements on hydrogen and deuterium targets. 
Table 6.7: The measured asymmetries, adjusted to the common $Q^{2}$ values, and the corresponding calculated asymmetry coefficients, for both the forward angle (hydrogen target) and the backward angle measurements (hydrogen and deuterium targets). The uncertainties are statistical, point-to-point systematic, and global systematic uncertainties, respectively.

\begin{tabular}{|ccc|cccc|}
\hline \hline Target Exp & $Q^{2}(\mathrm{GeV} / \mathrm{c})^{2}$ & Asymmetry $(\mathrm{ppm})$ & $\mathrm{a} 0$ & $\mathrm{a} 1$ & $\mathrm{a} 2$ & $\mathrm{a} 3$ \\
\hline \hline H Forward & 0.221 & $-4.72 \pm 0.36 \pm 0.21 \pm 0.23$ & -4.68 & 23.02 & 4.13 & 0.21 \\
H Back & 0.221 & $-11.10 \pm 0.86 \pm 0.27 \pm 0.43$ & -10.35 & 10.31 & 8.62 & 2.64 \\
D Back & 0.221 & $-17.07 \pm 0.81 \pm 0.42 \pm 0.21$ & -15.67 & 7.07 & 1.99 & 2.92 \\
H Forward & 0.628 & $-20.72 \pm 0.78 \pm 0.90 \pm 1.31$ & -23.68 & 79.39 & 42.87 & 2.46 \\
H Back & 0.628 & $-46.05 \pm 2.41 \pm 0.82 \pm 1.03$ & -38.28 & 21.57 & 62.84 & 12.10 \\
D Back & 0.628 & $-55.38 \pm 3.31 \pm 1.97 \pm 0.71$ & -53.29 & 12.12 & 12.49 & 9.50 \\
\hline \hline
\end{tabular}

\subsection{Results}

The extracted vector strange and axial form factors, using asymmetry measurements from both the forward angle and the backward angle $G^{0}$ experiments, are given in Table 6.8 .

Figure 6.4 shows the $G^{0}$ form factors plotted with theory calculations and other experimental results. The error bars represent the statistical and statistical plus point-to-point systematic uncertainties. The shaded bars located below each data point represent global systematic uncertainties $\left[\mathrm{Aac}^{+} 10\right]$.

For $G_{E}^{s}$ and $G_{M}^{s}$, the extraction from [LMRM07] (Low $Q^{2}$ fit) and the PVA4 experiment $\left[\mathrm{B}^{+} 09 \mathrm{~b}\right]$ are shown. Recent calculations from Adelaide $\left[\mathrm{LBC}^{+} 05, \mathrm{LBT}^{+} 06\right]$ and Kentucky $\left[\mathrm{D}^{+}\right.$09b] are also shown; for the Adelaide calculation, the uncertainties are smaller than the symbols. For $G_{A}^{e, T=1}$, results from the SAMPLE experiment [BPS05] are shown along with the calculation of Zhu, et al. [ZPHRM00]. The 


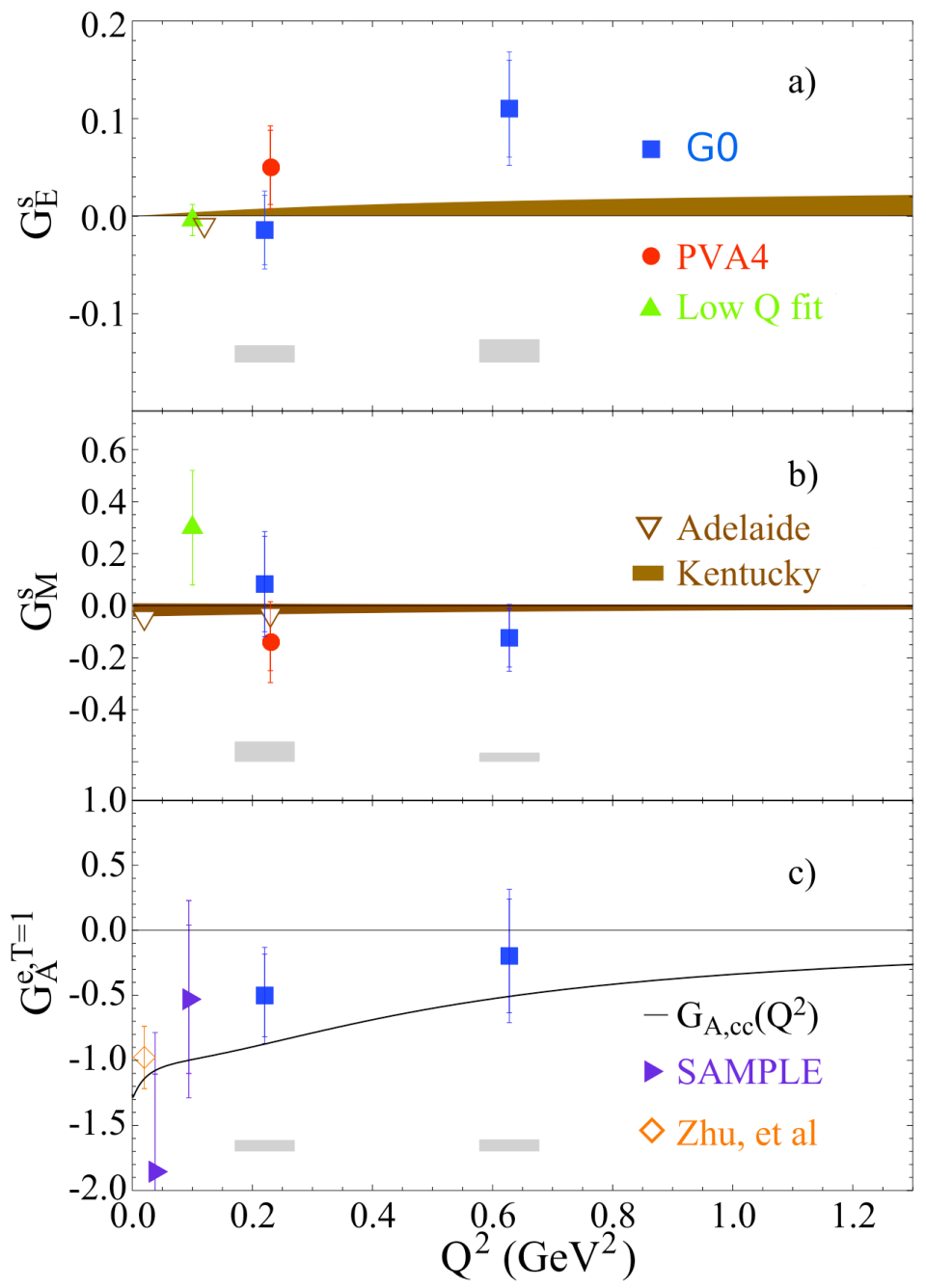

Figure 6.4: The form factors determined by the $G^{0}$ forward and backward angle measurements. Error bars show statistical and statistical plus point-to-point systematic uncertainties; shaded bars below corresponding points show global systematic uncertainties $\left(G^{0}\right)$. For $G_{E}^{s}$ and $G_{M}^{s}$, the extraction from [LMRMOr] (Low $Q^{2}$ fit) and the PVA4 experiment $\left[B^{+} 09 b\right]$ are shown. Recent calculations from Adelaide $\left[L B C^{+} 05, L_{B} T^{+} 06\right]$ and Kentucky $\left[D^{+} 09 b\right]$ are also shown. The Adelaide uncertainties are smaller than the symbols. For $G_{A}^{e, T=1}$, results from the SAMPLE experiment [BPS05] are shown with the calculation of Zhu, et al. [ZPHRM00] 
Table 6.8: Extracted vector strange and axial form factors obtained from the measurements taken during the $G^{0}$ forward angle and backward angle measurements. The first set of uncertainties are associated with the asymmetry measurement. The second set of uncertainties are related to the parameters used to extract the form factors from the measurement.

\begin{tabular}{|cc|c|}
\hline \hline Form Factor & $\begin{array}{c}\text { Uncertainty (asymmetry) } \\
\text { stat } \pm \text { pt-pt } \pm \text { global }\end{array}$ & $\begin{array}{c}\text { Uncertainty (parameters) } \\
\text { pt-pt } \pm \text { global }\end{array}$ \\
\hline \hline$G_{E}^{s}\left(0.221(\mathrm{GeV} / \mathrm{c})^{2}\right)$ & $-0.0142 \pm 0.0356 \pm 0.0182$ & $\pm 0.0176 \pm 0.0084 \pm 0.0026$ \\
$G_{M}^{s}\left(0.221(\mathrm{GeV} / \mathrm{c})^{2}\right)$ & $0.0834 \pm 0.1834 \pm 0.0855$ & $\pm 0.0781 \pm 0.0402 \pm 0.0167$ \\
$G_{A}^{e}\left(0.221(\mathrm{GeV} / \mathrm{c})^{2}\right)$ & $-0.5012 \pm 0.3171 \pm 0.1930$ & $\pm 0.0878 \pm 0.1040 \pm 0.0061$ \\
$G_{E}^{s}\left(0.628(\mathrm{GeV} / \mathrm{c})^{2}\right)$ & $0.1102 \pm 0.0488 \pm 0.0296$ & $\pm 0.0 .0237 \pm 0.0105 \pm 0.0023$ \\
$G_{M}^{s}\left(0.628(\mathrm{GeV} / \mathrm{c})^{2}\right)$ & $-0.1235 \pm 0.1095 \pm 0.0614$ & $\pm 0.0317 \pm 0.0155 \pm 0.0058$ \\
$G_{A}^{e}\left(0.628(\mathrm{GeV} / \mathrm{c})^{2}\right)$ & $-0.1973 \pm 0.4254 \pm 0.2568$ & $\pm 0.0949 \pm 0.0440 \pm 0.0027$ \\
\hline \hline
\end{tabular}

PVA4 result assumes a value for $G_{A}^{e(T=0)}$ calculated following the method of Zhu et al [ZPHRM00] using an axial mass of $\Lambda_{A}=1.032 \mathrm{GeV}$.

\subsubsection{Conclusions}

The parity-violating asymmetries in elastic electron-proton and quasi-elastic electron-deuteron scattering at $Q^{2}=0.22$ and $Q^{2}=0.63(\mathrm{GeV} / \mathrm{c})^{2}$ were measured. The asymmetries are sensitive to strange quark contributions to currents in the nucleon and the nucleon axial-vector current. This was the first time that $G_{E}^{s}$, $G_{M}^{s}$, and $G_{A}^{e}$ were all extracted from a common experiment. The results, shown in Table 6.8 and in Fig. 6.4, indicate that strange quarks make small contributions $(<$ $10 \%)$ to the charge and magnetic form factors of the nucleon for four-momentum transfers less than $0.63(\mathrm{GeV} / \mathrm{c})^{2}$. Although the total strange quark momentum measured in deep-inelastic scattering is approximately one half that of up and down 
sea quarks $\left[\mathrm{A}^{+} 00\right]$, the $G^{0}$ results suggest no significant spatial separation of the strange and anti-strange sea quarks, consistent with the small differences in their measured momentum distributions $\left[\mathrm{LNP}^{+} 07\right]$. The positive value of $G_{E}^{s}$ at $Q^{2}=$ $0.628(\mathrm{GeV} / \mathrm{c})^{2}$ reflects the positive values of the quantity $G_{E}^{s}+\eta G_{M}^{s}$ observed in the forward angle $G^{0}$ measurements $\left[\mathrm{A}^{+} 05\right]$. The values of $G_{A}^{e}$ measured during the $G^{0}$ experiment, are the first experimental indication of the $Q^{2}$ dependence of the nucleon anapole moment effects [HR00, MVvK00].

\subsection{Future Outlook}

Late in 2009, the HAPPEx collaboration finished taking a forward angle measurement at $Q^{2}=0.6(\mathrm{GeV} / \mathrm{c})^{2}$ using a $100 \mu \mathrm{A}, 3.4 \mathrm{GeV}$ polarized electron beam on a $25 \mathrm{~cm}$ long liquid hydrogen target. The precision of the anticipated results are for $\delta\left(G_{E}^{s}+0.48 G_{M}^{s}\right)=0.0070($ statistical $) \pm 0.0042$ (systematic) \pm 0.0079 (form factors) [Dal09], which is an improvement in precision of over $100 \%$ compared with the $G^{0}$ forward angle measurement at $0.62(\mathrm{GeV} / \mathrm{c})^{2}$. Additionally, PVA-4 at Mainz is analyzing data for a forward angle measurement also at $Q^{2}=0.6(\mathrm{GeV} / \mathrm{c})^{2}$, and a backward angle measurement at $Q^{2}=0.23(\mathrm{GeV} / \mathrm{c})^{2}$.

At the conclusion of HAPPEx and PVA4, a global analysis should be considered using all of the PVES world data. Because the form factor extraction is dependent on the choice of electromagnetic form factor and the electroweak radiative correction approach and parameters, a common analysis using all of the world data would be of interest. 
The $Q_{\text {weak }}$ Experiment, scheduled to begin running in the summer of 2010, continues the use of parity violating electron scattering on the proton to look for new physics. The goal of the $Q_{\text {weak }}$ collaboration is to make the first precision measurement of the proton's weak charge, $Q_{W}^{p}$, which is predicted by the Standard Model to be $Q_{W}^{p}=1-4 \sin ^{2} \theta_{W}$. The collaboration plans on a 2200 hour measurement of the parity violating asymmetry in elastic ep scattering at $Q^{2}=0.03(\mathrm{GeV} / \mathrm{c})^{2}$ employing $180 \mu \mathrm{A}$ of polarized beam on a $35 \mathrm{~cm}$ liquid hydrogen target in order to determine the proton's weak charge with a $4 \%$ combined statistical and systematic error $\left[\mathrm{A}^{+}\right]$. Any significant deviation of $\sin ^{2} \theta_{W}$ from the Standard Model prediction at low $Q^{2}$ would signal new physics, while agreement places new and significant constraints on possible Standard Model extensions. This measurement is now feasible because the hadronic uncertainties are significantly constrained as a result of the SAMPLE, HAPPEx, PVA4, and $G^{0}$ measurements.

Experimental programs are currently underway and planned for Brookhaven's Relativistic Heavy Ion Collider (RHIC) and electron-ion collider (EIC), CERN, DESY (Hamburg), SLAC and Jefferson Laboratory which include studies of the spin structure of the nucleon. Measurements of the polarized gluon density suggest that it is much too small to resolve the spin crisis $\left[\mathrm{A}^{+} 06 \mathrm{a}, \mathrm{LSS} 06\right]$. This implies that the partons possess orbital angular momentum, and it appears possible to estimate this, at least for quarks, via a study of deeply virtual Compton scattering on protons [Ji03].

By combining DIS data with the growing reservoir of data on semi-inclusinve DIS (SIDIS) it should become possible to learn about the polarized sea densities 
$\Delta \bar{u}$ and $\Delta \bar{d}$ and to resolve the present disagreement between DIS and SIDIS about the sign of the strange quark density $\Delta s(x)+\Delta \bar{s}(x)$ [KCL09]. These programs and others like them will continue to improve our understanding of the contributions of individual quarks and the virtual sea to the fundamental characteristics of nucleons. 


\section{Appendix A}

\section{Electromagnetic Radiative Corrections}

\section{A.1 Introduction}

Electron scattering provides a relatively clean probe of nuclear structure, but because scattered electrons radiate in the presence of nuclei or other electrons, there are associated non-trivial electromagnetic radiative effects that must be considered. An electron scattered from a nucleon will emit an infinite number of photons, most of which carry away almost no energy, so that the total energy of the emitted photons is finite [BN37]. It is much more probable for one photon to take up most of the

radiated energy than for an equal sharing by all of the photons [Tsa71]. Because of this, electromagnetic radiative corrections typically assume a one photon exchange between the electron current and the hadron current. This radiation is calculable in the framework of QED, based on knowledge of the single photon exchange, or Born approximation cross section. The probability of photon emission is proportional to the cross section for no photon emission, where the proportionality constant depends upon $Q^{2}, E_{s}$ (the incident electron energy), and $E_{p}$ (the outgoing electron energy) and therefore can be calculated and used to correct the cross section for these radiative effects [Tsa71].

It is useful to distinguish the processes by which the scattered electron loses energy. These energy losses can be considered as falling into two major categories. 
The first is when the radiation is only influenced by the scattering nucleus, and the second is when the radiation is produced in the field of another nucleus. Radiative corrections in the field of the scattering nucleus include bremsstrahlung (internal) and corrections due to the emission and reabsorption of virtual photons. These effects are proportional to the number of target scatterers or the target thickness. Processes that result in radiation that are produced in the field of a nucleus other than the scattering nucleus include ionization and bremsstrahlung (external). Two scatterers are necessary to produce external radiation (the target nucleus, and another nucleus), and so these effects are proportional to the square of the target thickness. The effect of these processes is to give the scattered electrons a distribution of initial and final energies for a given beam energy. The small energy losses associated with ionization tends to smear the elastic (or quasi-elastic) peak while external bremsstrahlung tends to move scattered electrons out of the "peak" to form a radiative "tail" region. In all cases, since the measured parity violating asymmetry is a function of $Q^{2}$, the magnitude of the asymmetry is reduced.

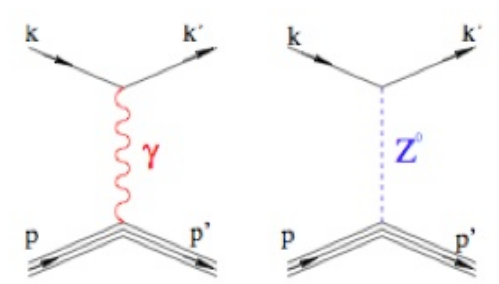

Figure A.1: First order diagrams for the elastic scattering of an electron from a nucleon.

The $G^{0}$ collaboration measured a physics asymmetry at backward angles, and this asymmetry requires a correction for radiative effects in order to extract the 


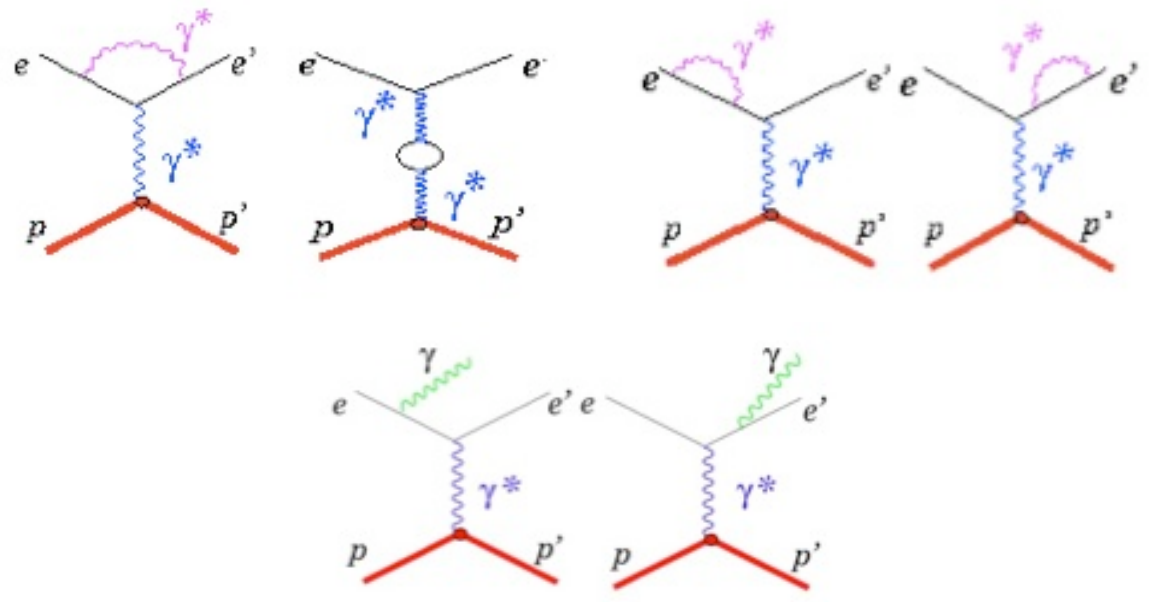

Figure A.2: Next to leading order Feynman diagrams necessary to calculate electromagnetic radiative effects

form factors of interest, $G_{E}^{s}, G_{M}^{s}$, and $G_{A}^{e(T=1)}$. To determine the correction, it is necessary to calculate two different parity violating asymmetries, the tree level asymmetry, $A_{\text {tree }}$, of single boson exchange, (Fig. A.1), and the asymmetry where the electromagnetic radiative effects, $A_{R C}$, (Fig. A.2), are included. The radiative correction, $R_{c}$ is simply the ratio of these two asymmetries

$$
R_{c}=\frac{A_{\text {tree }}}{A_{R C}}
$$

The corrections are calculated following the prescription laid out by Mo and Tsai [MT69], that was subsequently updated by Tsai [Tsa71], using a simulation technique employed by Spayde [Spa01]. The details are explained below.

\section{A.2 GEANT Monte Carlo Simulation}

GEANT3 is a FORTRAN based detector description and simulation tool created at CERN to model the passage of elementary particles through matter. The ra- 
diative corrections simulation code was written based on a code developed for the $G^{0}$ experiment using GEANT3 tools (G0GEANT, version May 2004) [W5094, Rol99]. G0GEANT includes a definition of the $G^{0}$ physical layout including the target, detectors, and spectrometer. The simulation is run after selecting initial parameters that include: beam energy, spectrometer current, target type, target temperature, $\max /$ min electron scattering angles (theta and phi), and the total number of Monte Carlo events. For each event, an electron is generated at a random point within the target volume, along the central axis of the target cylinder. The electron is given a random scattering direction in both theta and phi, and is assigned a random scattered energy. The electron is then propagated in the scattering direction through the target and then through the experimental area, which includes the spectrometer and all of the detectors. A particle making it into an active detector volume is called a "hit" and the information related to the hit, such as the flight time, detector, and energy, is stored along with other information for the event. In G0GEANT, each scintillator is an active detector volume, and more than one hit may be stored for a single event. In the actual experiment, a good electron event requires a coincidence of the front and back FPDs with a CED and a Čerenkov detector. The storage of event variables in GEANT makes it easy to analyze the Monte Carlo by making cuts on key parameters, variables, or detectors thereby eliminating any event that doesn't equate to a good experimental event.

Depending upon the type of process, elastic electron scattering, quasi-elastic deuterium scattering, etc., a cross section is calculated within G0GEANT, which is then used to weight each event to reflect that event's probability of occurrence. The 
event's weight can be expressed as

$$
\text { weight }=\frac{d \sigma}{d E d \Omega} \sin (\theta) \Delta_{v o l}
$$

where $\frac{d \sigma}{d E d \Omega}$ is the three-fold differential cross section, $\sin (\theta)$ is the Jacobian between $d \Omega$ and $d \theta d \phi$, and $\Delta_{v o l}$ is the constant phase space volume into which the events are generated. The phase space will change depending on what the "thrown" variables are within the Monte Carlo. In the case of elastic electron-proton scattering including radiative corrections (describe in detail below), the phase space is

$$
\Delta_{v o l}=\left(E_{p \max }-E_{p \min }\right)\left(\theta_{\max }-\theta_{\min }\right)\left(\phi_{\max }-\phi_{\min }\right) .
$$

The detector rate can be expressed as

$$
R=\mathrm{E} \times\left\langle\frac{d \sigma}{d E d \Omega}\right\rangle \sin (\theta) \Delta_{p h}
$$

where $\mathrm{E}$ is the luminosity,

$\mathrm{E}=$ number of incident electrons per second $\times$ number of nucleons in the target per $\mathrm{cm}^{2}$, and $\Delta_{p h}$ is the actual volume within $\Delta_{v o l}$ that is covered by the detector acceptance.

Because the events are uniformly thrown into the phase space, the ratio between $\Delta_{p h}$ and $\Delta_{v o l}$ is the same as the ratio between the number of good hits, $N_{\text {hits }}$, and the total number of generated events, $N_{\text {thrown }}$. Using this relationship, Eqn. A.4 becomes

$$
R=\mathrm{E} \times\left\langle\frac{d \sigma}{d E d \Omega} \sin (\theta)\right\rangle \Delta_{\text {vol }} \frac{N_{\text {hits }}}{N_{\text {thrown }}}=\mathrm{€} \times \frac{\Sigma_{i} w_{i}}{N_{\text {thrown }}}
$$

where $w_{i}$ is the weight associated with each event, and $\Sigma_{i}$ is the summation over all events. 
Similarly, the mean of any parameter, such as $Q^{2}$ or asymmetry is calculated as

$$
<P>=\frac{\sum_{i} w_{i} P_{i}}{\sum_{i} w_{i}}
$$

where $\mathrm{P}$ is the parameter of interest.

\section{A.3 Details of the Calculation for Hydrogen}

For each event, it is the electron's randomly selected initial position in the target that determines its initial energy, $E_{s}$ at the interaction point. In the absence of ionization, $E_{s}$ would be the incident beam energy, however the passage of the electron through the target material results in an energy loss. The incident electron energy is calculated as

$$
E_{s}=E_{\text {beam }}-\Delta_{H_{2}}\left(E_{s}, t_{H_{2}}\right)-\Delta_{A l}\left(E_{s}, t_{A l}\right)-\Delta_{H e}\left(E_{s}, t_{H e}\right)
$$

where $\Delta_{H e}$ is the loss from the electron passing through the helium cell, $\Delta_{A l}$ is the energy loss due to ionization in a thickness of aluminium, $t_{A l}$, which includes both the target entrance window and the helium cell window, and $\Delta_{H_{2}}$ is the energy lost in passing through the hydrogen target cell to the interaction point. The calculation for the energy due to ionization accounts for the usual Bethe-Bloch stopping power and density effect corrections. The fluctuations around the mean energy loss are included and the straggling was computed assuming a Landau distribution. Whether the simulation is run with or without radiative effects, ionization losses in the target prior to the scattering event are included. This ensures that the two simulations used for the correction have the same mean incident beam energy. 
For the "tree"-level simulation, the cross section for elastic electron-proton scattering is used [HM84]:

$$
\frac{d \sigma_{\text {elas }}}{d \Omega}\left(E_{s}, \theta\right)=\left(\frac{\alpha^{2}}{4 E_{s}^{2} \sin ^{4}\left(\frac{\theta}{2}\right)}\right) \frac{E^{\prime}}{E_{s}}\left[\frac{G_{E}^{2}+\tau G_{M}^{2}}{1+\tau} \cos ^{2}\left(\frac{\theta}{2}\right)+2 \tau G_{M}^{2} \sin ^{2}\left(\frac{\theta}{2}\right)\right]
$$

where $E^{\prime} / E_{s}$ arises from the recoil of the target and constrains the scattered energy to a maximum energy equal to $E^{\prime}$,

$$
E^{\prime}=\frac{E_{s}}{1+\frac{2 E_{s}}{M_{p}}} \sin ^{2}\left(\frac{\theta}{2}\right)
$$

where $\tau=Q^{2} / 4 m^{2}$ and $M_{p}$ is the mass of the proton [HM84].

The parity violating asymmetry [Nap91],

$$
A_{p v}=\left(\frac{-G_{F} Q^{2}}{4 \sqrt{2} \pi \alpha}\right) \frac{\epsilon G_{E}^{\gamma} G_{E}^{z}+\tau G_{M}^{\gamma} G_{M}^{z}-\left(1-4 \sin ^{2} \theta_{W}\right) \epsilon^{\prime} G_{M}^{\gamma} G_{A}^{e}}{\epsilon\left(G_{E}^{\gamma}\right)^{2}+\tau\left(G_{M}^{\gamma}\right)^{2}}
$$

is calculated as a function of $E_{s}$ and $\theta$, where

$$
\begin{aligned}
\epsilon & =\left(1+2(1+\tau) \tan ^{2}(\theta / 2)\right)^{-1} \\
\epsilon^{\prime} & =\sqrt{\tau(1+\tau)\left(1-\epsilon^{2}\right)}
\end{aligned}
$$

From the event asymmetry calculation, a weighted mean asymmetry value for the elastically scattered electrons, $A_{\text {tree }}$, is determined using Eqn. A.6.

To calculate $A_{R C}$, a similar procedure is followed as described for the "tree" calculation, except that corrections are made to the elastic cross sections to include radiative effects. Following the prescription of Mo and Tsai, [MT69], there are two cross section formulas; one for the "peak" region, where only small energy losses have occurred, and one for the "tail" region, where the majority of events fall with 
any significant radiation. Determining whether an event in the Monte Carlo is in the "peak" or "tail", requires knowing the final scattered energy of the electron and comparing this to the final energy of an electron that is elastically scattered. This is accomplished by randomly selecting the final scattered energy, $E_{p}$ and comparing this energy to the energy of an elastically scattered electron, given the event's value for $E_{s}$ and $\theta$ using Eqn. A.9.

If the randomly scattered energy, $E_{p}$, is within $1 \mathrm{MeV}$ of the energy for an elastically scattered electron, there is no significant energy loss and the event is considered a "peak" event. The selection of $1 \mathrm{MeV}$ is somewhat arbitrary and widening or narrowing this energy window, $\Delta E$, where $R \Delta E$ is the maximum energy of photons which can be emitted along the incident electron direction, will have little impact on the result as long as the cross section at incident energies $E_{s}$ and $E_{s}-R \Delta E$ varies by less than $10 \%$ [Tsa71].

The expression for the radiated "peak" cross section calculation [Tsa71] is :

$$
\frac{d \sigma_{\text {peak }}}{d \Omega}=G\left(\theta, t_{H_{2}}\right) \frac{d \sigma_{\text {elas }}}{d \Omega} \times e^{\delta}\left(\frac{R \Delta E}{E_{s}}\right)^{b t}\left(1-\frac{\xi}{\Delta E}\right)
$$

where $\frac{d \sigma_{\text {elas }}}{d \Omega}$ is the elastic cross section (Eqn. A.8), $\delta$ is given by Eqn.(II.6) in [MT69], and

$$
G\left(\theta, t_{H_{2}}\right)=1+0.5772 b_{H_{2}} t_{H_{2}}+\frac{\alpha}{\pi}\left[\frac{1}{6} \pi^{2}-\Phi\left(\cos ^{2}\left(\frac{\theta}{2}\right)\right)\right]
$$

with the Spence function, $\Phi$, defined as $\Phi(x) \equiv \int_{0}^{x} \frac{-\ln |1-y|}{y} d y . \xi$ is a parameter in 
Landau straggling and is given by

$$
\begin{aligned}
\xi & =0.154\left(\frac{z}{a}\right) T x_{0} \\
T & =\text { target thickness in units of radiation length } \\
x_{0} & =\text { unit radiation length in } \mathrm{gm} / \mathrm{cm}^{2}
\end{aligned}
$$

The atomic number and the atomic weight are $z$ and $a$, respectively. Additionally,

$$
b=\frac{4}{3}\left\{1+\frac{1}{9}[(z+1) /(z+\eta)]\left[\ln \left(183 z^{-1 / 3}\right]^{-1}\right\}\right.
$$

where $\eta$ is due to the bremsstrahlung emission and is given by

$$
\eta=\frac{\ln \left(1440 a^{-2 / 3}\right)}{\ln \left(183 z^{-1 / 3}\right)}
$$

The term $b t=b_{H_{2}} t_{H_{2}}+b_{A l} t_{A l}+b_{H e} t_{H e}$ with $t_{H_{2}}$ describing the position of the scattering event within the target volume along the longitudinal axis and is equivalent to the amount of target material the electron passed through prior to the scattering event, whereas $t_{A l}$ and $t_{H e}$ are the thickness of the aluminum windows and the helium cell, respectively. The term " $t$ " therefore represents the total thickness of all of the materials the electron passes through prior to scattering. In Tsai, the target is assumed to be comprised of a single material, and the position of the scattering event within the material is always approximated as $\frac{T}{2}$.

As mentioned previously, the maximum allowed energy of a real photon emitted in the direction of the incident electron is $R \Delta E$ [Tsa71]:

$$
R \equiv \frac{\omega_{s}}{\omega_{p}}
$$

where $\omega_{s}$ is the maximum energy of a photon which can be emitted along the direction of the incident electron, and $\omega_{p}$ is the maximum photon energy which 
can be emitted along the direction of the outgoing electron.[Tsa71]

$$
\begin{aligned}
\omega_{s} & =\frac{1}{2} \frac{u^{2}-M_{P}^{2}}{M-E_{p}(1-\cos \theta)}, \\
\omega_{p} & =\frac{1}{2} \frac{u^{2}-M_{P}^{2}}{M+E_{s}(1-\cos \theta)} .
\end{aligned}
$$

In these expressions,

$$
u^{2}=2 m_{e}^{2}+M_{p}^{2}-Q^{2}+2 M_{p}\left(E_{s}-E_{p}\right)
$$

is the missing mass squared.

The calculation of the "peak" cross section in Tsai also includes a term for external bremsstrahlung losses after the interaction, but those are already calculated within the GEANT simulation so are omitted in Eqn. A.13. An asymmetry is calculated for the event using Eqn. A.10.

If $E_{p}$ is less than $E^{\prime}-\Delta E$, then significant energy losses have occured either before or after the scattering event, and a calculation of the cross section for an event that is part of the radiative tail is required [Tsa71].

$$
\begin{aligned}
\frac{d \sigma_{\text {tail }}}{d \Omega d E_{p}}=v_{s}^{b\left(t+t_{r}\right)} & {\left[\left(\frac{d \sigma_{\text {elas }}\left(E_{s}-\omega_{s}\right)}{d \Omega}\right) \frac{M+\left(E_{s}-\omega_{s}\right)(1-\cos \theta)}{M-E_{p}(1-\cos \theta)}\right.} \\
& \times\left(\left(t+t_{r}\right) \frac{b}{\omega_{s}} \phi\left(v_{s}\right)+\frac{\xi}{2 \omega_{s}^{2}}\right)+\frac{d \sigma_{\text {elas }}\left(E_{s}\right)}{d \Omega}\left(b t_{r} \frac{1}{\omega_{p}} \phi\left(v_{p}\right)\right]
\end{aligned}
$$

where

$$
\begin{aligned}
v_{s} & =\frac{\omega_{s}}{E_{s}} \\
v_{p} & =\frac{\omega_{p}}{E_{p}+\omega_{p}} \\
t_{r} & =\frac{1}{b_{H_{2}}} \frac{\alpha}{\pi}\left[\ln \left(\frac{Q^{2}}{m_{e}^{2}}-1\right)\right]
\end{aligned}
$$


Eqn. A.22 includes effects due to ionization, internal and external bremsstrahlung, and virtual photons. The effect of the internal bremsstrahlung is roughly the same as that given by two external radiators with one placed before and one after the scattering, each of thickness $t_{r}$, given in radiation lengths. The function $\phi(v)$ gives the shape of the bremsstrahlung spectrum normalized such that $\phi(0)=1$. [Tsa71]

Eqn. A.22 accounts for the fact that an electron with a final energy $E_{p}$ may have emitted a photon with energy $\omega_{s}$ before the parity violating interaction making its initial energy, $E_{s}-\omega_{s}$, or instead, a photon with energy $\omega_{p}$, may have been emitted after the interaction. In that case the electron's initial energy is $E_{s}$.

The parity violating asymmetry for a "tail" event is formed in a similar manner as the cross section, by taking a linear combination of the asymmetries for the two different cases of initial energy, $A_{p v}\left(E_{s}-\omega_{s}, \theta\right)$ and $A_{p v}\left(E_{s}, \theta\right)$, weighted by the corresponding cross sections and the radiative effects correction terms corresponding to the cross section. Additionally, the asymmetry calculation contains terms correcting for the depolarization of the incident electron due to a spin flip, caused by the emitted photons. The asymmetry calculation for a "tail" event is:

$$
\begin{aligned}
& A_{R C^{\text {tail }}}\left[\frac{d \sigma_{\text {tail }}}{d \Omega d E_{p}}\right]=v_{s}^{b\left(t+t_{r}\right)}\left[\left(A_{p v}\left(E_{s}-\omega s\right) \frac{d \sigma_{\text {elas }}\left(E_{s}-\omega_{s}\right)}{d \Omega}\right) \frac{M+\left(E_{s}-\omega_{s}\right)(1-\cos \theta)}{M-E_{p}(1-\cos \theta)}\right. \\
& \times\left(\left(t\left(1-D_{\text {tot }}^{e x t}\right)+t_{r}\left(1-D_{\text {tot }}^{\text {int }}\right)\right) \frac{b}{\omega_{s}} \phi\left(v_{s}\right)+\frac{\xi}{2 \omega_{s}^{2}}\right)+A_{p v}\left(E_{s}\right) \frac{d \sigma_{\text {elas }}\left(E_{s}\right)}{d \Omega}\left(b t_{r} \frac{1}{\omega_{p}} \phi\left(v_{p}\right)\right] .
\end{aligned}
$$

The term $D^{e x t}$ represents the depolarization of an electron because of external 
bremsstrshlung as calculated by Olsen and Maximon [OM59]:

$$
D^{e x t}\left(E_{s}, E_{p}, \zeta_{1}\right)=\frac{\left(E_{s}-E_{p}\right)^{2}\left[\Psi_{1}-\zeta_{1 z}^{2}\left(\psi_{1}-\frac{2}{3} \psi_{2}\right)\right]}{\left(E_{s}^{2}-E_{p}^{2}\right) \Psi_{1}-\frac{2}{3} E_{s} E_{p} \psi_{2}}
$$

where $\zeta_{1}$ is the unit spin vector of the initial electron (therefore $\zeta_{1 z}^{2}=1$ ). At high energies, where complete screening is assumed:

$$
\begin{aligned}
\psi_{1} & =4\left[\ln \left(183 Z^{\frac{1}{3}}\right)-f(Z)\right] \\
\psi_{2} & =\psi_{1}-\frac{2}{3} \\
f(z) & =(z \alpha)^{2} \sum_{n=1}^{\infty} \frac{1}{n\left(n^{2}+(z \alpha)^{2}\right.}
\end{aligned}
$$

and $D_{t o t}^{e x t}=D_{H_{2}}+D_{A l}+D_{H e}$.

The depolarization due to internal bremsstrahlung was derived from the work of Kuchto and Shumeiko [KS83], who calculated the cross section for the scattering of longitudinally polarized electrons from longitudinally polarized nucleons. The effects of internal bremsstrahlung were included in their calculation. The result applicable to accounting for depolarization during polarized electron scattering from nucleons is this calculation [Spa01]

$$
\left(1-D^{i n t}\right) \equiv \frac{\left[E_{s}^{2}+\left(E_{s}^{\prime}\right)^{2}\right] \ln \left(\frac{Q^{2}}{m_{e}^{2}}\right)-2\left(E_{s}^{2}+E_{s} E_{s}^{\prime}-\left(E_{s}^{\prime}\right)^{2}\right)}{\left[E_{s}^{2}+E_{s} E_{s}^{\prime}\right] \ln \left(\frac{Q^{2}}{m_{e}^{2}}\right)-2 E_{s} E_{s}^{\prime}}
$$

where $E_{s}^{\prime}=E_{s}-\omega_{s}$. It should be noted that this is not strictly a simple depolar-

ization factor, because $\left(1-D^{i n t}\right) \geq 1$ for small $\omega_{s}$ [Spa01].

\section{A.4 Details of the Calculation for Deuterium}

The deuterium calculation followed the same basic process as the hydrogen radiative effects calculation, except for three significant differences: 1) the unradiated 
cross section and asymmetry calculations, 2) an additional "thrown" variable for the Monte Carlo, and 3), inclusion of a non-zero nucleon momentum in the target nucleon's 4-vector.

For the unradiated cross section calculation, $\frac{d \sigma_{e l a s}(E)}{d \Omega}$ in Eqns A.13, A.22, and A.26, and the unradiated asymmetry calculation, $A_{p v}$ in Eqn. A.26, a combination of models was used. The $G^{0}$ backangle experiment had access to calculations from R. Schiavilla [Sch06], [SCP04], for both the deuterium cross section and asymmetry. The calculations are based on the AV18 nucleon-nucleon interaction model. For the low energy radiative corrections, the full 2-body version of the calculation was used. In this version, the neutron and proton wave functions are obtained from solutions of the Schrodinger equation in the continuum, so they fully account for interaction effects in the final state. The meson exchange current (MEC) contributions in the electroweak current operator were also included [SCP04]. These MEC corrections are not only in the electromagnetic current, but also in the neutral weak current. The 2-body calculation contained a small error with one of the relativistic corrections. This error had a very minor (less than $2 \%$ ) effect on the asymmetry calculation for the low energy $(362 \mathrm{MeV})$ data. For the high energy $(687 \mathrm{MeV})$ data, the error was more significant, so a partial wave impulse approximation (PWIA) analysis was used instead. For the PWIA, final-state interactions were ignored and only one-body terms were included in the currents [SCP04].

Both calculations require significant computer processing time, so look-up tables were created for use by the Monte Carlo. Each of the two kinematic points had tables containing three energies and four angles. Intermediate values were calcu- 
lated through a straight line interpolation in $\vec{q}$ and $\omega$. There were many instances however, where the Monte Carlo event fell outside of the region covered by the R. Schiavilla tables. In those instances the asymmetry was calculated using a nearly static calculation where the neutron and proton are treated as free, non-interacting particles and the asymmetries are added incoherently:

$$
A_{D}=\frac{\sigma_{p} A_{P}+\sigma_{N} A_{n}}{\sigma_{D}}
$$

The deuterium cross section was calculated using a "y-scaling" model. "Y-scaling" assumes that the quasi-elastic scattering process is basically the ejection of a single nucleon allowing the cross section to be written as the product of a kinematic factor $\mathrm{C}$, a single-nucleon cross section, $\sigma_{e N}$, and a universal scaling function of only one variable, $F(y)$, rather than as a function of $\mathrm{q}$ and $\omega$ [Dow87],

$$
\frac{d^{2} \sigma}{d \Omega d E_{p}}=C \sigma_{e N} F(y)
$$

The scaling variable, $y$, was defined by Kawazoe et al. [KTM75] as the component of the nucleon mometum along the direction of momentum transfer:

$$
y=\frac{\vec{q} \cdot \vec{p}_{N}}{q}
$$

The variable, $y$, samples the momentum distribution of the nucleons parallel to $\vec{q}$, and should not depend directly on the energy or scattering angle of the electron. Both the static asymmetry calculation and the y-scale cross section calculation were normalized to match the R. Schiavilla value at any given $Q^{2}$.

As in the case of hydrogen, the electron scattering angles, $\theta$ and $\phi$, are "thrown" as well as the final scattered energy, $E_{p}$. $E_{p}$ determines the amount 
of energy lost in radiation for a given event. For deuterium, an additional energy variable is necessary, because the cross section is a function of the struck nucleon's momentum distribution. For the Monte Carlo, $\omega$ was "thrown", where $\omega=E_{s}-E_{f}$ which provided $E_{f}$ as the final, un-radiated electron energy. In the GEANT simulation, $E_{f}$ is a function of the nucleon's momentum whereas $E_{p}$ is only a function of the radiated photon energy.

When the momentum of the target nucleon can be taken to be zero, as in the case of elastic scattering from hydrogen targets, the calculation of the missing mass (Eqn. A.21) and photon energies (Eqns A.19 and A.20) is much simplified. However, the nucleons in deuterium have a non-zero momentum that must be considered. For the Monte Carlo, a simplification was made by assigning the magnitude of the struck nucleon's momentum as $y$ (Eqn. A.34), taking the mass of the nucleon as the mass of a proton, and by taking the direction of the nucleon's momentum to be the same as the direction of momentum transfer. This changed Eqn. A.21 to:

$$
\begin{aligned}
U^{2} & =2 m_{e}^{2}+M_{p}^{2}-Q^{2}+2\left(E_{s}-E_{p}\right) \sqrt{M_{p}^{2}+y^{2}} \\
& -2\left[E_{s}^{2}+E_{p} E_{f}-E_{s}\left(E_{f}+E_{p}\right) \cos \theta\right] \frac{y}{\sqrt{2 E_{s} E_{f}(1-\cos \theta)+\omega^{2}}}
\end{aligned}
$$

and Eqns. (A.19 and A.20) to:

$$
\begin{aligned}
\omega_{s} & =\frac{u^{2}-M_{p}^{2}}{2\left(E_{h}-E p(1-\cos \theta)-\left(\vec{P}_{p} \cdot \hat{s}\right)\right)}, \\
\omega_{p} & =\frac{u^{2}-M_{p}^{2}}{2\left(E_{h}+E_{s}(1-\cos \theta)-\left(\vec{P}_{p} \cdot \hat{p}\right)\right)}
\end{aligned}
$$


where

$$
\begin{aligned}
& \vec{P}_{p} \cdot \hat{s}=y \frac{\left(E_{s}-E_{f} \cos \theta\right)}{\left|q_{3}\right|}, \\
& \vec{P}_{p} \cdot \hat{p}=y \frac{\left(E_{s} \cos \theta-E_{f}\right)}{\left|q_{3}\right|}
\end{aligned}
$$

and

$$
\begin{aligned}
E_{h} & =\sqrt{M_{p}^{2}+y^{2}}, \\
\left|q_{3}\right| & =\sqrt{2 E_{s} E_{f}(1-\cos \theta)+\omega^{2}} .
\end{aligned}
$$

\section{A.5 Results}

After a simulation is run, the data are analyzed by making "cuts" on the data in order to discard bad events. Bad events are events that fail to strike each of the appropriate detectors (FPD front and back, CED, and Čerenkov) in the proper order, that fail to "fire" the appropriate Cerenkov detector, or that are in any way unphysical. The struck CED-FPD pair provides coarse angle and momentum information allowing for separation of inelastic and elastic (or quasi-elastic) events. A cut on the amount of energy deposited in the Čerenkov detector separates the electrons from the pions. After the cuts are applied, good events are binned by FPD-CED detector pair, and then a Čerenkov efficiency is applied to the rate [Ver08a, Ver08b]. An asymmetry is calculated for good elastic electron or quasi-elastic electron events using Eqn. A.6.

For most of the experiment, the electronics required that two of the four Cerenkov photomultiplier tubes fire, in order for the event to count as a good electron event. This condition is referred to as "multiplicity 2". For a brief time period 
the experiment ran with multiplicity 3 , or the requirement for three of the four PMTs to fire.

Once the simulations are run, there are two methods of applying the radiative correction to the experimental data. The first method is to apply the correction calculated for $a_{0}$ to the measured physics asymmetry. The second method takes advantage of the fact that Eqn. A.10 can be rewritten as:

$$
A_{p v}(q, \omega, \theta)=a_{0}+a_{1} G_{E}^{s}+a_{2} G_{M}^{s}+a_{3} G_{A}^{e(T=1)}+a_{4} G_{A}^{s}
$$

If the experimental physics asymmetry is broken down into its constituent parts, a correction can be calculated and applied to each coefficient. A comparison was made between the two methods and the difference was less than $0.05 \%$ to the final corrected asymmetry. It was decided to apply the correction for $a_{0}, R_{c}\left(a_{0}\right)$, to the physics asymmetry.

Typical behavior of $Q^{2}$ and $a_{0}$ with and without radiative corrections applied are shown in Figs. A.3 and A.4. These plots show the impact of an electron radiating photons, and why this must be accounted for in any precision electron scattering experiment. The plots reflect the impact when radiation routinely lowers the scattered electron energy, thereby reducing an event's $Q^{2}$ and therefore the magnitude of the asymmetry. Eight million simulation events were run for each simulation. This resulted in an average statistical certainty of \pm 0.002 for $A_{R C}$. Other parameters were analyzed to determine their contribution to the uncertainty in the asymmetry correction. These parameters were:

- $3 \%$ reduction in target density 

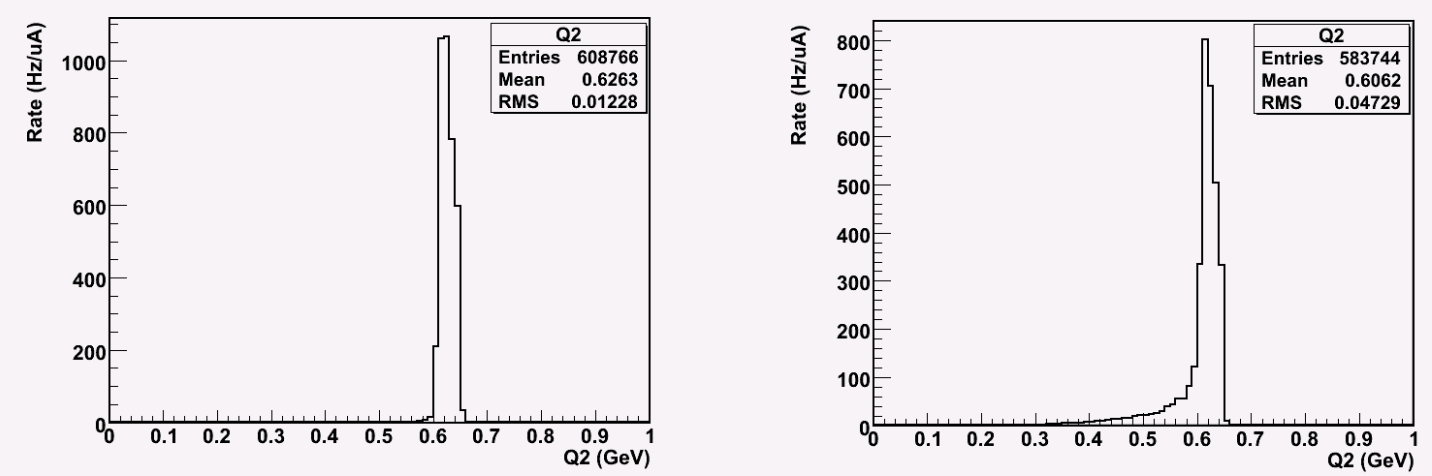

Figure A.3: $Q^{2}$ vs normalized event rates for incident beam energy $685 \mathrm{MeV}$ and a hydrogen target. The plot on the left is with no radiative effects, the plot on the right includes radiative corrections.
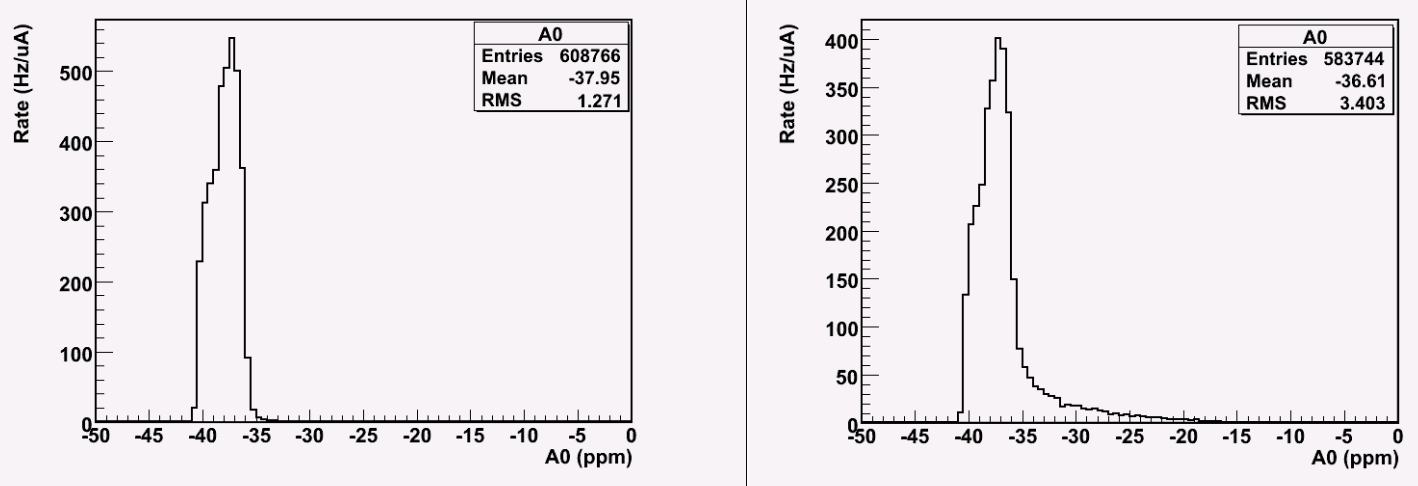

Figure A.4: $a_{0}$ vs normalized event rates for incident beam energy $685 \mathrm{MeV}$ and a hydrogen target. The plot on the left is with no radiative effects, the plot on the right includes radiative corrections.

- $\Delta \mathrm{E}$ of $2 \mathrm{MeV}$ vs $1 \mathrm{MeV}$

- $1 \%$ change in the spectrometer's magnetic field

- change in deuterium cross section and asymmetry model (Y-scale/static asymmetry calculation plus R. Schiavilla versus only using Y-scale/static asymmetry calculation)

- Using a momentum mass model for the deuterium radiative corrections vs 
assigning a nucleon momentum

The only parameter change that resulted in a change in the correction outside of the statistical uncertainty, was the choice of deuterium model. This induced an additional uncertainty of \pm 0.003 . The total uncertainty in the radiative correction for deuterium is \pm 0.0036 , or $11 \%$ uncertainty on the correction. For hydrogen, the uncertainty is \pm 0.002 or $5.5 \%$ uncertainty on the correction.

The corrections for all of the asymmetry coefficients, listed by incident beam energy, are shown in Table A.1 for deuterium targets, and Table A.2 for hydrogen targets. 
Table A.1: Deuterium Electromagnetic Radiative Corrections. Multiplicity $=2$ unless otherwise noted.

\begin{tabular}{|c|c|c|c|}
\hline \multicolumn{4}{|c|}{ target/beam energy = D 363.05} \\
\hline Asym. Coef. & $A_{\text {tree }}($ ppm $)$ & $A_{R C}(p p m)$ & $R_{c}$ \\
\hline $\mathrm{a} 0$ & -15.492 & -15.019 & $1.032 \pm 0.004$ \\
\hline a1 & 7.158 & 7.089 & $1.010 \pm 0.001$ \\
\hline a2 & 1.966 & 1.883 & $1.044 \pm 0.005$ \\
\hline a3 & 2.882 & 2.801 & $1.029 \pm 0.003$ \\
\hline a4 & 0.251 & 0.243 & $1.033 \pm 0.004$ \\
\hline \multicolumn{4}{|c|}{ target/beam energy= D 689.42} \\
\hline Asym. Coef. & $A_{\text {tree }}(\mathrm{ppm})$ & $A_{R C}(\mathrm{ppm})$ & $R_{c}$ \\
\hline $\mathrm{a} 0$ & -52.445 & -50.797 & $1.032 \pm 0.004$ \\
\hline a1 & 13.755 & 13.609 & $1.011 \pm 0.001$ \\
\hline $\mathrm{a} 2$ & 13.666 & 12.994 & $1.052 \pm 0.006$ \\
\hline a3 & 11.273 & 10.851 & $1.039 \pm 0.004$ \\
\hline $\mathrm{a} 4$ & 0.983 & 0.947 & $1.038 \pm 0.004$ \\
\hline \multicolumn{4}{|c|}{ target/beam energy= D 689.61} \\
\hline Asym. Coef. & $A_{\text {tree }}(\mathrm{ppm})$ & $A_{R C}(\mathrm{ppm})$ & $R_{c}$ \\
\hline $\mathrm{a} 0$ & -53.537 & -52.487 & $1.035 \pm 0.004$ \\
\hline a1 & 13.752 & 13.623 & $1.010 \pm 0.001$ \\
\hline $\mathrm{a} 2$ & 13.684 & 12.969 & $1.055 \pm 0.006$ \\
\hline a3 & 11.284 & 10.834 & $1.042 \pm 0.005$ \\
\hline $\mathrm{a} 4$ & 0.905 & 0.9452 & $1.041 \pm 0.005$ \\
\hline \multicolumn{4}{|c|}{ target/beam energy $=$ D 689.61 Multiplicity $=3$} \\
\hline Asym. Coef. & $A_{\text {tree }}(\mathrm{ppm})$ & $A_{R C}(\mathrm{ppm})$ & $R_{c}$ \\
\hline $\mathrm{a} 0$ & -52.347 & -50.586 & $1.035 \pm 0.004$ \\
\hline a1 & 13.798 & 13.662 & $1.010 \pm 0.001$ \\
\hline $\mathrm{a} 2$ & 13.627 & 12.912 & $1.055 \pm 0.006$ \\
\hline a3 & 11.246 & 10.796 & $1.042 \pm 0.005$ \\
\hline $\mathrm{a} 4$ & 0.980 & 0.94190 & $1.041 \pm 0.004$ \\
\hline
\end{tabular}


Table A.2: Hydrogen Electromagnetic Radiative Corrections. Multiplicity $=2$.

\begin{tabular}{|c|c|c|c|}
\hline \multicolumn{4}{|c|}{ target/beam energy= H 361.90} \\
\hline Asym. Coef. & $A_{\text {tree }}(\mathrm{ppm})$ & $A_{R C}(\mathrm{ppm})$ & $R_{c}$ \\
\hline $\mathrm{a} 0$ & -10.434 & -10.064 & $1.037 \pm 0.002$ \\
\hline a1 & 10.200 & 10.110 & $1.009 \pm 0.001$ \\
\hline $\mathrm{a} 2$ & 8.781 & 8.388 & $1.047 \pm 0.003$ \\
\hline a3 & 2.684 & 2.587 & $1.037 \pm 0.002$ \\
\hline $\mathrm{a} 4$ & 1.203 & 1.156 & $1.037 \pm 0.002$ \\
\hline \multicolumn{4}{|c|}{ target/beam energy $=$ H 684.86} \\
\hline Asym. Coef. & $A_{\text {tree }}(p p m)$ & $A_{R C}(p p m)$ & $R_{c}$ \\
\hline $\mathrm{a} 0$ & -37.951 & -36.612 & $1.037 \pm 0.002$ \\
\hline a1 & 21.760 & 21.542 & $1.010 \pm 0.001$ \\
\hline $\mathrm{a} 2$ & 62.523 & 59.198 & $1.056 \pm 0.003$ \\
\hline a3 & 12.042 & 11.508 & $1.046 \pm 0.003$ \\
\hline $\mathrm{a} 4$ & 5.400 & 5.159 & $1.046 \pm 0.003$ \\
\hline \multicolumn{4}{|c|}{ target/beam energy $=\mathrm{H} 685.57$} \\
\hline Asymmetry & $A_{\text {tree }}($ ppm $)$ & $A_{R C}(p p m)$ & $R_{c}$ \\
\hline $\mathrm{a} 0$ & -38.026 & -36.697 & $1.036 \pm 0.002$ \\
\hline a1 & 21.783 & 21.557 & $1.010 \pm 0.001$ \\
\hline $\mathrm{a} 2$ & 62.735 & 59.420 & $1.056 \pm 0.003$ \\
\hline a3 & 12.074 & 11.544 & $1.046 \pm 0.003$ \\
\hline a4 & 5.412 & 5.174 & $1.046 \pm 0.003$ \\
\hline
\end{tabular}




\section{Appendix B}

\section{Luminosity Monitors}

Luminosity is the interaction rate of the electron beam with the target, per unit cross section. Eight luminosity detectors (Lumis) were placed at very forward beam angles in order to check for beam induced false asymmetries and to monitor target density fluctuations. The Lumis capitalize on the fact that forward beam angles see very high rates of scattered electrons, with a small expected asymmetry for a longitudinally polarized electron beam.
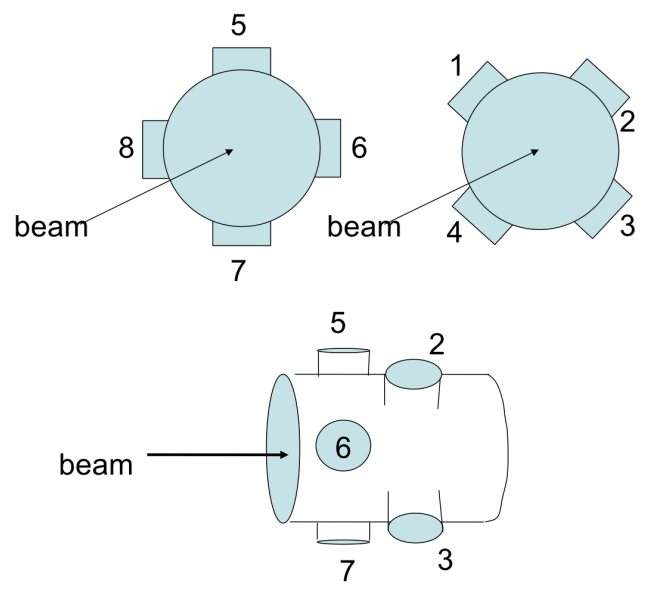

Figure B.1: An illustration of the placement of the Lumis in relationship to one another and the beam line.

\section{B.1 Detectors}

The Lumis are Čerenkov detectors comprised of a synthetic quartz scintillator, copper wire mesh which served as a light attenuator, and a photomultiplier tube 
with a low gain base. They were configured in two sets of four detectors placed around the beam line as shown in Fig. B.1. The set of detectors numbered 1-4, are $377.0 \mathrm{~cm}$ downstream from the center of the target and sitting at a laboratory angle of $3.74^{\circ}$. The second set of detectors, numbered 5-8, are $354.12 \mathrm{~cm}$ downstream from the center of the target and sitting at a laboratory angle of $3.98^{\circ}$. The

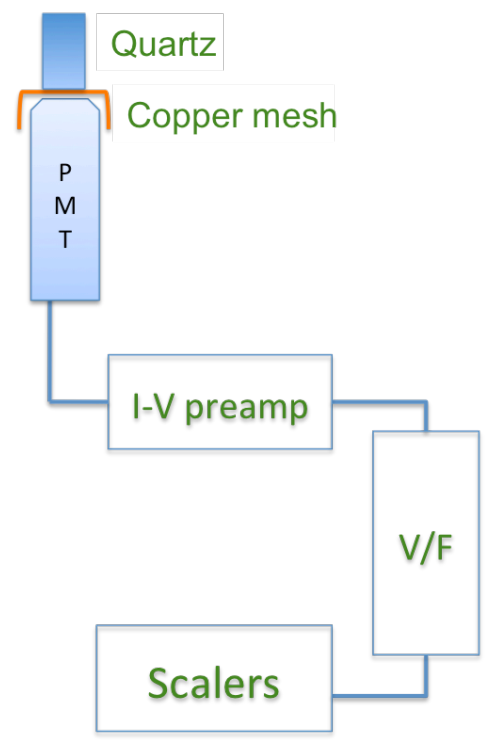

Figure B.2: An illustration of the configuration of one Lumi detector, not drawn to scale. The quartz scintillator, copper mesh, and PMT, for each detector were located in the experimental hall. The electronics, which included the I-V preamp, $V-F$ convertors, and the scalers were located in the $G^{0}$ electronics cage.

scintillators are Spectrosil 2000, a synthetic fused silica, from Saint Gobain Quartz. Each crystal measures $3.56 \mathrm{~cm} \times 3.56 \mathrm{~cm} \times 7.62 \mathrm{~cm}$ and all six faces are finished with an optical polish. The quartz has an index of refraction, $n=1.47$, resulting in a threshold Čerenkov velocity of $\beta_{t}=0.68$. Because the scattered electrons are relativistic, $\beta \sim 1$, they will emit photons as they traverse the quartz.

The photomultiplier tube gain was set so that for each $\mu A$ of beam current 
there was $0.1 \mu A$ of anode current $\left(I_{a}\right)$. This resulted in nominal anode currents between 2 and $6 \mu A$ during the backward angle measurement. The anode currents from the individual photomultiplier tubes, (see Fig. B.2), were sent into commercial current-to-voltage (I-V) pre-amplifiers. Lumi 1-4 used Hamamatsu C7319 preamplifiers and Lumi 5-8 used a PMT4 preamplifier from Advanced Research Instruments. The preamps were set at $1.0 \mathrm{~V} / \mu \mathrm{A}$ resulting in signals seen by the voltage-to-frequency ( V-F) converters between 2-6 V. The voltage signal was then digitized by the V-F converters, which were manufactured by TRIUMF, with the output sent to scalers that were read out for each helicity state (1/30 s). A different set of scalers was used for each beam helicity state.

Situated between the quartz scintillator and the quartz face of the photomultiplier tube, were a few (1-3) layers of fine copper wire mesh which were used to reduce the light seen in the photomultiplier tube. Each layer of mesh had a $30 \%$ transmission rate.

The quartz and the photomultiplier tube were aligned so that the long central axis of each was collinear. The assembled detectors were placed in aluminum cups that extended into the beam pipe with the quartz end of the detector placed closest to the beam, and the long axis of the detector aligned in a radial direction in reference to the beam line, as shown in Fig. B.3. The radius of the beam pipe was $30.5 \mathrm{~cm}$. The radial distance from the bottom of the aluminum cup to the center of the beam line was $19.8 \mathrm{~cm}$. The thickness of the aluminum cup was $0.16 \mathrm{~cm}$. The quartz was sitting $\sim 0.5 \mathrm{~cm}$ above the bottom of the the aluminum cup. The photomultiplier tubes were Hamamatsu R375's, which are 51 mm (2 inch) diameter, 


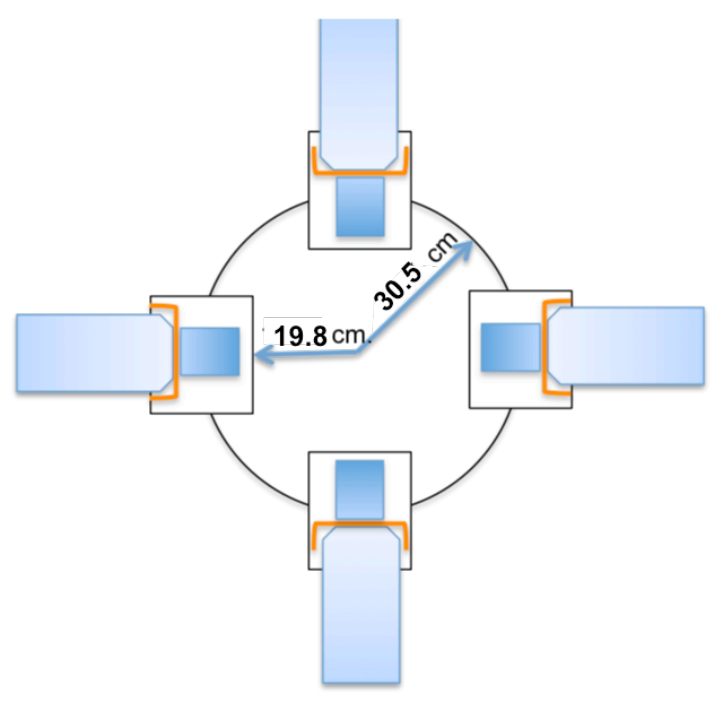

Figure B.3: An illustration of the placement of one set of four Lumis around the beam pipe viewed from the target. A cross section of the downstream beam pipe, the detectors and the aluminum cups that hold the detectors are shown. The beam was centered on the axis of the beam pipe, and $19.8 \mathrm{~cm}$ from the bottom of each aluminium cup.

10-stage, head-on, with a multi-alkali photocathode and a response range of 160 $850 \mathrm{~nm}$.

\section{B.2 Geometry and Kinematics}

As mentioned previously in Sec. 4.1 and Sec. 4.18, the $G^{0}$ experiment took backward angle measurements from both liquid hydrogen $\left(19^{\circ} \mathrm{K}\right)$ and liquid deuterium $\left(21^{\circ} \mathrm{K}\right)$ targets. The target cell was $20 \mathrm{~cm}$ in length, with thin aluminum entrance and exit windows on each end. A helium cell for cooling was located immediately prior to the target entrance window. The helium cell also had an aluminum entrance window that the beam passed through on its way to the liquid target. The total thickness of the three aluminum windows was $0.0483 \mathrm{~cm}$. 
When polarized electrons were scattered from the liquid hydrogen target, the dominant scattering processes at forward angles where the Lumis were located were Møller (e-e), electron-proton (e-p), and elastic electron-aluminum (e-Al) (see Figs. B.4 and B.5. From the luminosity, cross section, and the Lumi solid angle, the predicted yield as a function of laboratory angle for each of these processes can be calculated. The Lumi solid angle is the area of the scintillator exposed to the target, or $3.56 \mathrm{~cm} \times 7.62 \mathrm{~cm}=27.13 \mathrm{~cm}^{2}$, divided by the square of the distance from the target to the detectors. The solid angle for detectors $1-4$ was $0.187 \mathrm{mrad}$ and for Lumis 5-8, $0.212 \mathrm{mrad}$. The luminosity is the number of electrons per second multiplied by the number of scattering centers per unit area. The luminosity values for all of the $G^{0}$ backangle measurement's beam current and target combinations is shown in Table B.1.

Table B.1: Luminosity values for all of the target and beam current combinations used during the $G^{0}$ backward angle measurement.

\begin{tabular}{|c|c|c|}
\hline \hline Target & Beam Current $(\mu A)$ & Luminosity $\left(\mathrm{cm}^{-2} \mathrm{~s}^{-1}\right)$ \\
\hline \hline $\mathrm{H}$ & 60 & $3.21 \times 10^{38}$ \\
$\mathrm{Al}$ & 60 & $6.76 \times 10^{35}$ \\
$\mathrm{D}$ & 35 & $2.20 \times 10^{38}$ \\
$\mathrm{Al}$ & 35 & $3.95 \times 10^{35}$ \\
$\mathrm{D}$ & 20 & $1.25 \times 10^{38}$ \\
$\mathrm{Al}$ & 20 & $2.25 \times 10^{35}$ \\
\hline \hline
\end{tabular}

The cross sections can be calculated using expressions from $\left[\mathrm{C}^{+} 97\right]$ for the Møller scattering, [HM84] for e-p and e-neutron, and [Won90] for elastic e-Al. For deuterium, a simple linear combination of the proton and neutron cross sections was 
used to estimate quasi-elastic scattering.

Table B.2: Predicted luminosity monitor yields and asymmetries by reaction channel and luminosity monitor for a hydrogen target. Beam energy is $360 \mathrm{MeV}$ and beam current is $60 \mu \mathrm{A}$.

\begin{tabular}{|c|c|c|c|c|}
\hline \multicolumn{3}{|c|}{ Hydrogen Beam energy $=360 \mathrm{MeV}$ Beam current $=60 \mu \mathrm{A}$} \\
\hline \hline \multirow{2}{*}{ Channel } & \multicolumn{2}{|c|}{$\theta_{\text {lab }}=3.74^{\circ}$} & \multicolumn{2}{|c|}{$\theta_{\text {lab }}=3.98^{\circ}$} \\
\cline { 2 - 5 } & Rate $(\mathrm{GHz})$ & Asymmetry $(\mathrm{ppm})$ & Rate $(\mathrm{GHz})$ & Asymmetry $(\mathrm{ppm})$ \\
\hline \hline Møller & 7.19 & 0.0022 & 6.61 & 0.0020 \\
e-p & 2.08 & -0.0035 & 1.60 & -0.0040 \\
e-Al & 0.75 & 0.054 & 0.58 & 0.062 \\
\hline \hline total & 10.02 & 0.0049 & 8.79 & 0.0049 \\
\hline
\end{tabular}

Table B.3: Predicted luminosity monitor yields and asymmetries by reaction channel and luminosity monitor for a hydrogen target. Beam energy is $685 \mathrm{MeV}$ and beam current is $60 \mu \mathrm{A}$.

\begin{tabular}{|c|c|c|c|c|}
\hline \multicolumn{4}{|c|}{ Hydrogen Beam energy $=685 \mathrm{MeV}$ Beam current $=60 \mu \mathrm{A}$} \\
\hline \hline \multirow{2}{*}{ Channel } & \multicolumn{2}{|c|}{$\theta_{\text {lab }}=3.74^{\circ}$} & \multicolumn{2}{|c|}{$\theta_{\text {lab }}=3.98^{\circ}$} \\
\cline { 2 - 5 } & Rate $(\mathrm{GHz})$ & Asymmetry $(\mathrm{ppm})$ & Rate $(\mathrm{GHz})$ & Asymmetry $(\mathrm{ppm})$ \\
\hline \hline Møller & 5.32 & 0.0029 & 5.14 & 0.0026 \\
e-p & 0.57 & -0.013 & 0.44 & -0.015 \\
e-Al & 0.21 & 0.11 & 0.16 & 0.22 \\
\hline \hline total & 6.10 & 0.0051 & 5.74 & 0.0073 \\
\hline
\end{tabular}

The result of folding the cross sections with the luminosity and Lumi solid angle for both the high and low energy runs on the hydrogen target is shown in Fig. B.4 and for deuterium in Fig. B.5. At the forward laboratory angles of $3.74^{\circ}$ and $3.98^{\circ}$ where the Lumis were located, Møller scattering was the dominant process, but electrons from either elastic e-p or quasi-elastic deuterium (e-n) scattering, as well as a small rate from elastic e-Al scattering were also seen. The actual rates along with the associated asymmetries are shown in Tables B.2 to B.5. The total 

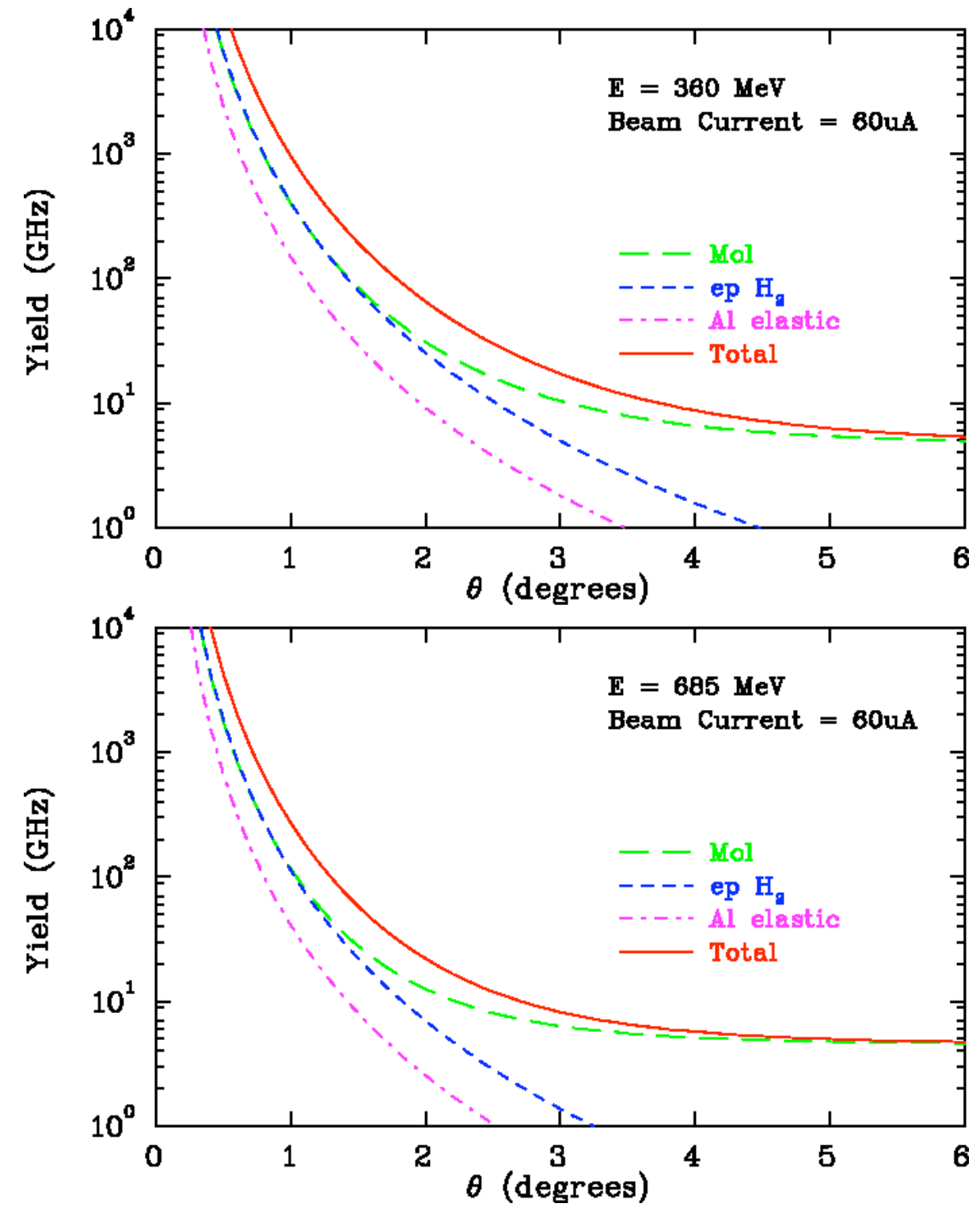

Figure B.4: Electron yield versus laboratory scattering angle for the Lumi solid angle for a hydrogen target with aluminum target windows, shown by reaction channel. Processes include Møller, e-p, and elastic e-Al scattering. 

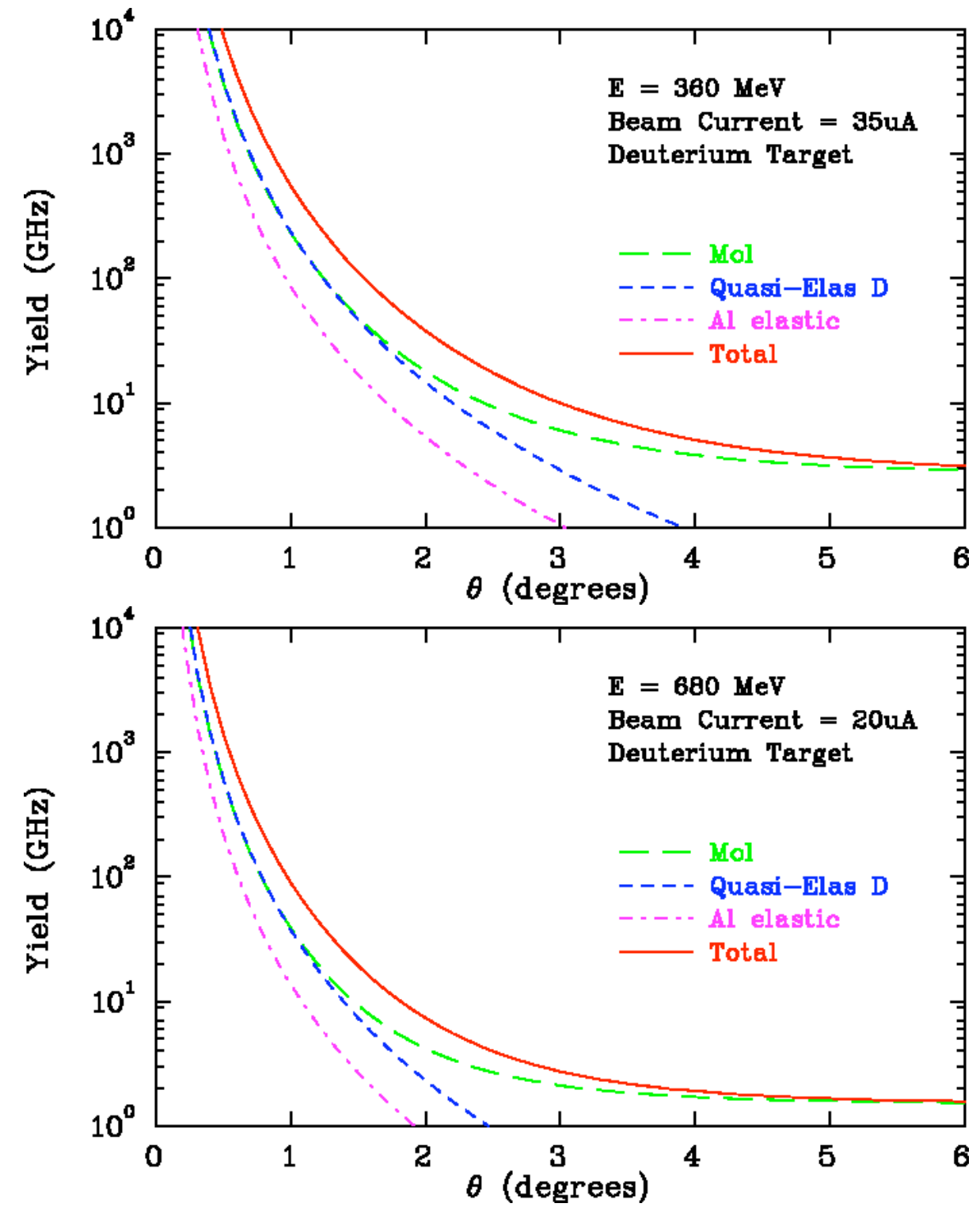

Figure B.5: Electron yield versus laboratory scattering angle for the Lumi solid angle for a deuterium target with aluminum target windows, shown by reaction channel. Processes include Møller, e-n, and elastic e-Al scattering. 
Table B.4: Predicted luminosity monitor yields and asymmetries by reaction channel and luminosity monitor for a deuterium target. Beam energy is $360 \mathrm{MeV}$ and beam current is $35 \mu \mathrm{A}$.

\begin{tabular}{|c|c|c|c|c|}
\hline \multicolumn{2}{|c|}{ Deuterium Beam energy $=360 \mathrm{MeV}$ Beam current $=35 \mu \mathrm{A}$} \\
\hline \hline \multirow{2}{*}{ Channel } & \multicolumn{2}{|c|}{$\theta_{\text {lab }}=3.74^{\circ}$} & \multicolumn{2}{|c|}{$\theta_{\text {lab }}=3.98^{\circ}$} \\
\cline { 2 - 5 } & Rate $(\mathrm{GHz})$ & Asymmetry $(\mathrm{ppm})$ & Rate $(\mathrm{GHz})$ & Asymmetry $(\mathrm{ppm})$ \\
\hline \hline Møller & 4.91 & 0.0022 & 4.55 & 0.0020 \\
e-n & 1.42 & -0.0036 & 1.10 & -0.0041 \\
e-Al & 0.44 & 0.054 & 0.34 & 0.062 \\
\hline \hline total & 6.77 & 0.0044 & 5.99 & 0.0043 \\
\hline
\end{tabular}

Table B.5: Predicted luminosity monitor yields and asymmetries by reaction channel and luminosity monitor for a deuterium target. Beam energy is $685 \mathrm{MeV}$ and beam current is $20 \mu \mathrm{A}$.

\begin{tabular}{|c|c|c|c|c|}
\hline \multicolumn{2}{|c|}{ Deuterium Beam energy $=685 \mathrm{MeV}$ Beam current $=20 \mu \mathrm{A}$} \\
\hline \hline \multirow{2}{*}{ Channel } & \multicolumn{2}{|c|}{$\theta_{\text {lab }}=3.74^{\circ}$} & \multicolumn{2}{|c|}{$\theta_{\text {lab }}=3.98^{\circ}$} \\
\cline { 2 - 5 } & Rate $(\mathrm{GHz})$ & Asymmetry $(\mathrm{ppm})$ & Rate $(\mathrm{GHz})$ & Asymmetry $(\mathrm{ppm})$ \\
\hline \hline Møller & 2.10 & 0.0029 & 2.03 & 0.0026 \\
e-n & 0.23 & -0.013 & 0.18 & -0.015 \\
e-Al & 0.07 & 0.11 & 0.05 & 0.22 \\
\hline \hline total & 2.40 & 0.0045 & 2.26 & 0.0060 \\
\hline
\end{tabular}


expected electron yield for each detector was between 2 and $10 \mathrm{GHz}$.

The average number of photoelectrons generated from an incident electron, $N_{\text {p.e. }}$, is given by the following expression:

$$
N_{p . e .}=L \frac{\alpha^{2} z^{2}}{r_{e} m_{e} c^{2}} \int \epsilon(E) \sin ^{2} \theta_{c}(E) d E
$$

where $\mathrm{L}$ is the path length in the radiator, $\epsilon(E)$ is the efficiency for collecting the Čerenkov light and converting it into photoelectrons, $z$ is the charge $(z=1$ for an electron) and $\alpha^{2} z^{2} / r_{e} m_{e} c^{2}=370 \mathrm{~cm}^{-1} \mathrm{eV}^{-1}$ [AoPDG08]. For the Hamamatsu R375 photomultiplier tube, the spectral response is centered on wavelengths between 175 $\mathrm{nm}$ and $550 \mathrm{~nm}$ (equivalent to an energy range of $1.13 \mathrm{eV}$ to $0.36 \mathrm{eV}$ ) and the average quantum efficiency is $\sim 15 \%$. The photocathode collection efficiency was estimated to be $\sim 60 \%$. This results in $N_{\text {p.e. }}=48$. This number does not include the attenuation when fine gauge wire mesh was placed between the quartz and the photomultiplier tube in the final design. Combining this result with the lowest expected incident electron rate of $2 \mathrm{GHz}$, and a typical photomultiplier tube gain of approximately $10^{5}$, the expected anode current for this detector configuration is $\sim 2$ $\mathrm{mA}$, which is much too high for routine operations. A unique detector design was required to reduce the gain and achieve a reasonable value for the anode current.

When the detectors were designed, the experiment was to run at a higher beam current, and the predicted rate was actually $\sim 20 \mathrm{GHz}$. During the forward angle runs, the measured anode currents were 40 times higher than predicted [Liu06] due largely to a significant secondary particle rate. Taking into account simulated and predicted rates for the backward angle measurement and the difference between the 
same calculations and actual rates for the forward angle measurement, the Lumi electron rates were expected to be 20 to 25 times the rate seen during the forward angle measurement.

For a given photomultiplier tube, the gain can be parameterized by:

$$
G=\beta\left(V^{\alpha}\right)^{n}
$$

where $\beta$ is a proportionality constant, $\alpha$ is a coefficient determined by the dynode material and geometric structure, $V$ is the potential difference between dynodes, and $n$ is the number of dynodes. Using the gain vs supply voltage chart supplied by Hamamatsu, the R375 photomultiplier tube has $\alpha=0.80$ and $\beta=0.09$. The gain of the photomultiplier tube is directly related to the voltage applied across the dynodes. Lowering the voltage reduces the gain and therefore reduces the anode current. However, if the voltage between dynodes is too low, non-linearity arises due to space charge effects. Additionally, the electric field between dynodes needs to be maintained above a certain level in order to maintain proper focusing of the cascading electrons [Leo94]. The decision was made to change the number of active dynodes from the manufacturer's recommended 10 to only four (five had been used during the forward angle measurement) and to maintain a constant inter-dynode voltage across the stages, to produce a robust, linear, low-gain response detector, (see Fig. B.6). The expected gain versus high voltage setting for the Hamamatsu R375 photomultiplier tube, using the custom designed base is shown in Fig. B.7.

With any base, the gain is lowered (raised) by reducing (increasing) the high 


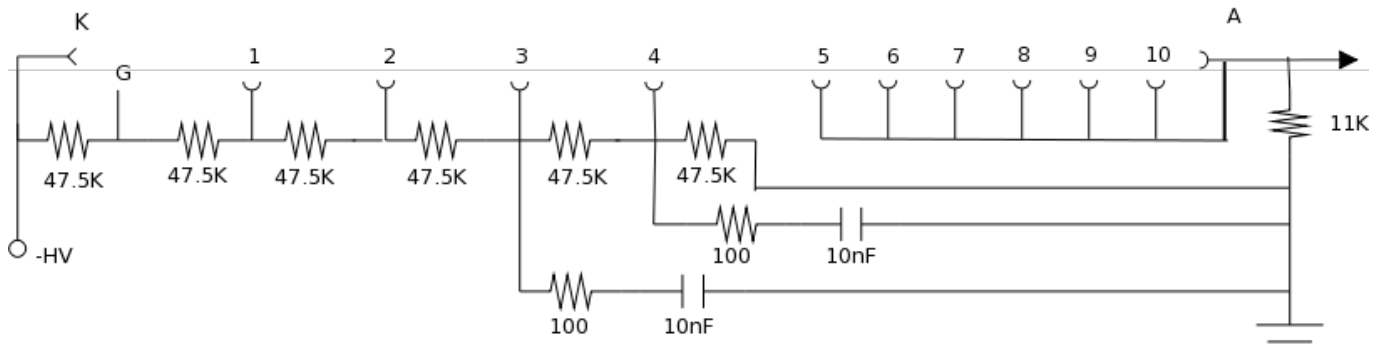

Figure B.6: Customized base designed for the Hamamatsu R375 photomultiplier tube. Only the first four stages are active. The signal is extracted at the 5th dynode stage. The resistors are in ohms and kilohms ( $k)$.

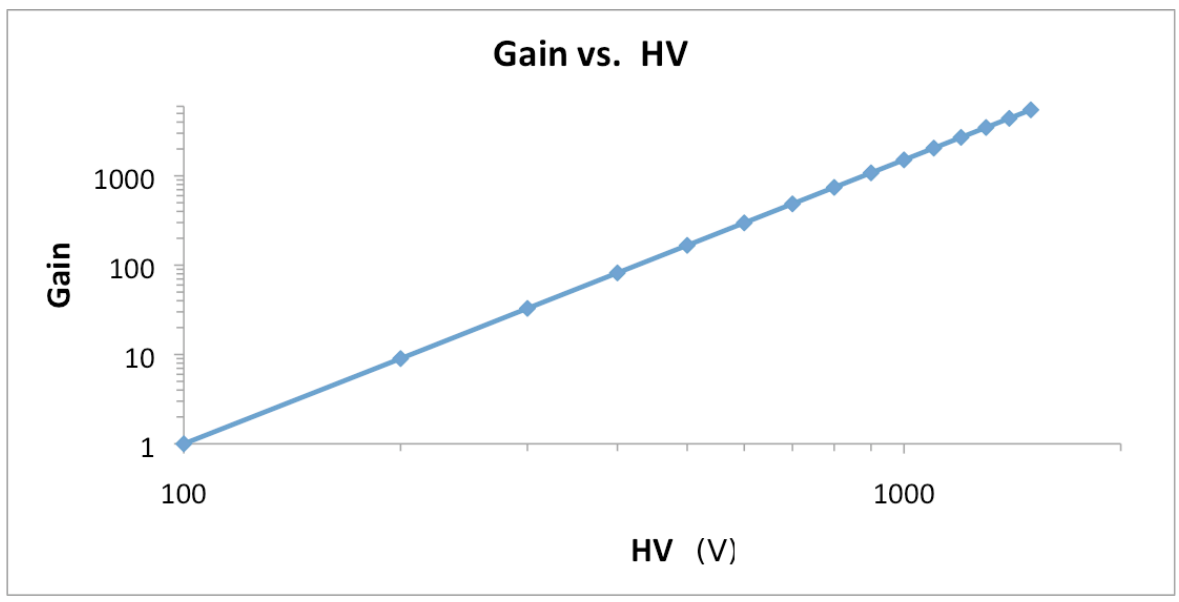

Figure B.7: The estimated gain versus high voltage for the Hamamatsu R375 photomultiplier tube with a customized low gain base. 
voltage. However, the inter-dynode voltage had to be over $50 \mathrm{~V}$ in order to maintain proper focusing of the cascading electrons. Even using only four active dynodes and

a high voltage setting of $250 \mathrm{~V}$, the predicted anode current was still too high. To reduce the current, copper wire mesh with $30 \%$ transmission was placed between the scintillator and the photomultiplier tube. The sole purpose of the mesh was to reduce the number of Čerenkov photons reaching the photocathode.

Calculations showed that two layers of mesh should be optimal $(\sim 9 \%$ transmission), but because the Lumis were located in an area difficult to access, six of the eight Lumis had two layers of mesh, while Lumi 2 had three layers of mesh and Lumi 4 had only one layer of mesh. Both Lumi 2 and Lumi 4 were the easiest Lumis to access and would provide additional information if two layers of mesh was either too many or too few. Initial testing of the Lumis showed that all of the detectors were able to run with the amount of copper mesh initial placed in the detectors with high voltage settings ranging from $210 \mathrm{~V}$ to $275 \mathrm{~V}$.

\section{B.3 Detector Linearity}

The detectors were built with as large a dynamic range as possible, to allow for electron rates higher or lower than predicted. This required the detectors to provide a linear response for $\mathrm{HV}$ setting from $200 \mathrm{~V}$ to the maximum of $1500 \mathrm{~V}$ with the expectation that the HV setting for each detector would be below $300 \mathrm{~V}$.

The linearity of the Lumis is shown in Fig. B.8 for a hydrogen target. There is a small increase in the normalized yield at $\sim 25 \mu \mathrm{A}$ of beam current most likely due 
to a poor monitor calibration or noise. Above $35 \mu \mathrm{A}$ of beam current, the difference in linearity was at most $0.5 \% \pm 0.7 \%$. When the lower beam current data is included the worst case has the normalized rate varying by $1.1 \% \pm 0.5 \%$.

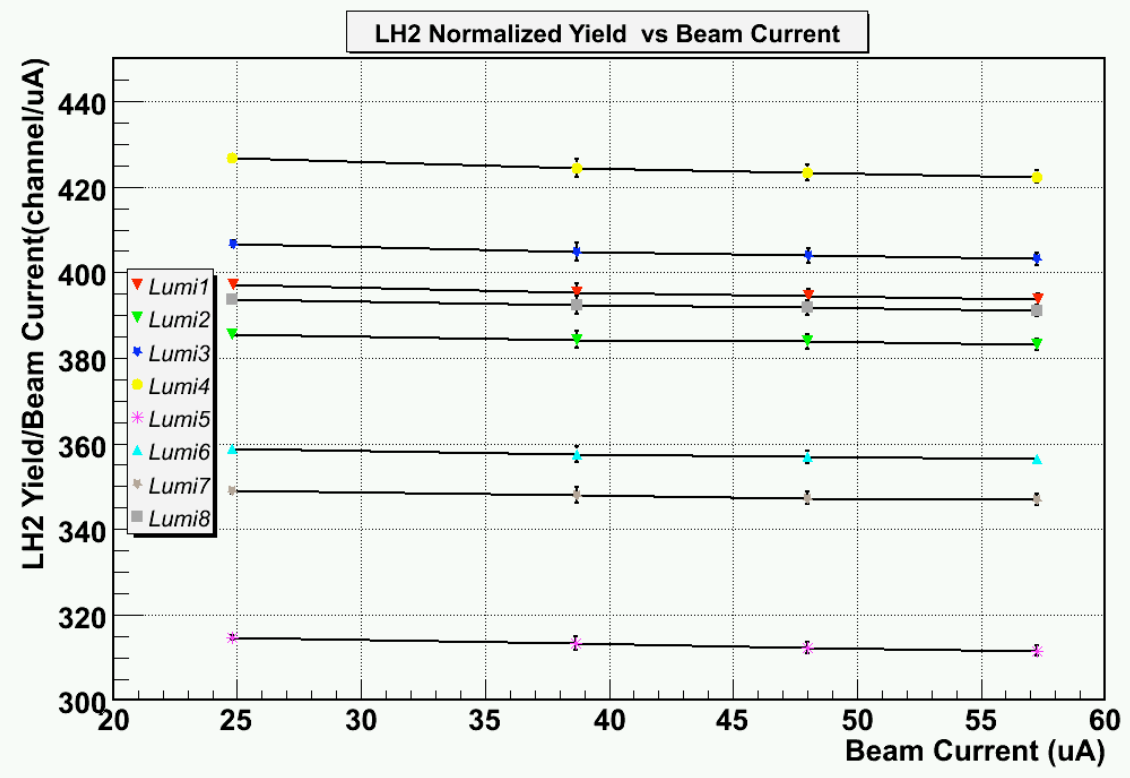

Figure B.8: Beam current normalized yields versus beam current for a hydrogen target with a beam energy of $687 \mathrm{MeV}$.

\section{B.4 Asymmetry Widths and Target Density Fluctuations}

Precision measurements of parity violation in electron scattering from liquid targets are sensitive to any time-dependent fluctuations in the target density. As discussed in Sec. 4.1, the $G^{0}$ experiment measured an asymmetry by measuring detector yields for both the + and - helicity states of the polarized electron beam. To do this, the helicity was physically "flipped" every $1 / 30$ of a second, defined as a "macropulse" (MPS). Four consecutive MPS form a "quartet" of either + - - + or -++- . A beam current normalized yield for each MPS was determined, $Y_{i}$, and 
the asymmetry calculated quartet-by-quartet using the following expression:

$$
A=H \frac{Y_{1}+Y_{4}-\left(Y_{2}+Y_{3}\right)}{Y_{1}+Y_{2}+Y_{3}+Y_{4}}
$$

where $H=1(-1)$ depending on the helicity state of the first MPS. The quartet asymmetries are then averaged over the run.

If the liquid target begins to boil, the effect will most probably be localized starting at the surfaces of the heated target windows. The rate at which heat is conducted away will depend on whether the boiling at the window is nucleate or makes a transition to film boiling (where the window is in contact with vapor rather than liquid) [DA03].

If the target density fluctuates from quartet to quartet, it results in fluctuations in the scattering rate where the statistical precision of the asymmetry could be degraded beyond Poisson statistics. If the fluctuations are slower than $1 / 30$ sec, then the fluctuations will tend to cancel within the quartet. Much faster fluctuations will average out over the MPS window, so it is only the density fluctuations on the time scale of one MPS that are of real concern [DA03]. It should be noted that helicity-correlated target density changes are a different effect, and may be removed via linear regression.

The width of the measured asymmetry can be decomposed as

$$
W_{\text {meas }}=\sqrt{W_{\text {stat }}^{2}+W_{\text {boil }}^{2}+W_{\text {noise }}^{2}} \text {. }
$$

where $W_{\text {boil }}$ is the contribution to the width due to target density fluctuations, $W_{\text {noise }}$ is the contribution due to electronic noise from detector and amplifier components, and $W_{\text {stat }}$ is the statistical width of the asymmetry distribution, determined from 
counting statistics. $W_{\text {stat }} \propto 1 / \sqrt{N}$ where $N$ is the total number of counts in a quartet. Noise was not measured during the backward angle measurement, but an estimate of the noise can be made by considering a solid target where the measured asymmetry width can be expressed as

$$
W_{\text {meas }}=\sqrt{W_{\text {stat }}^{2}+W_{\text {noise }}^{2}}
$$

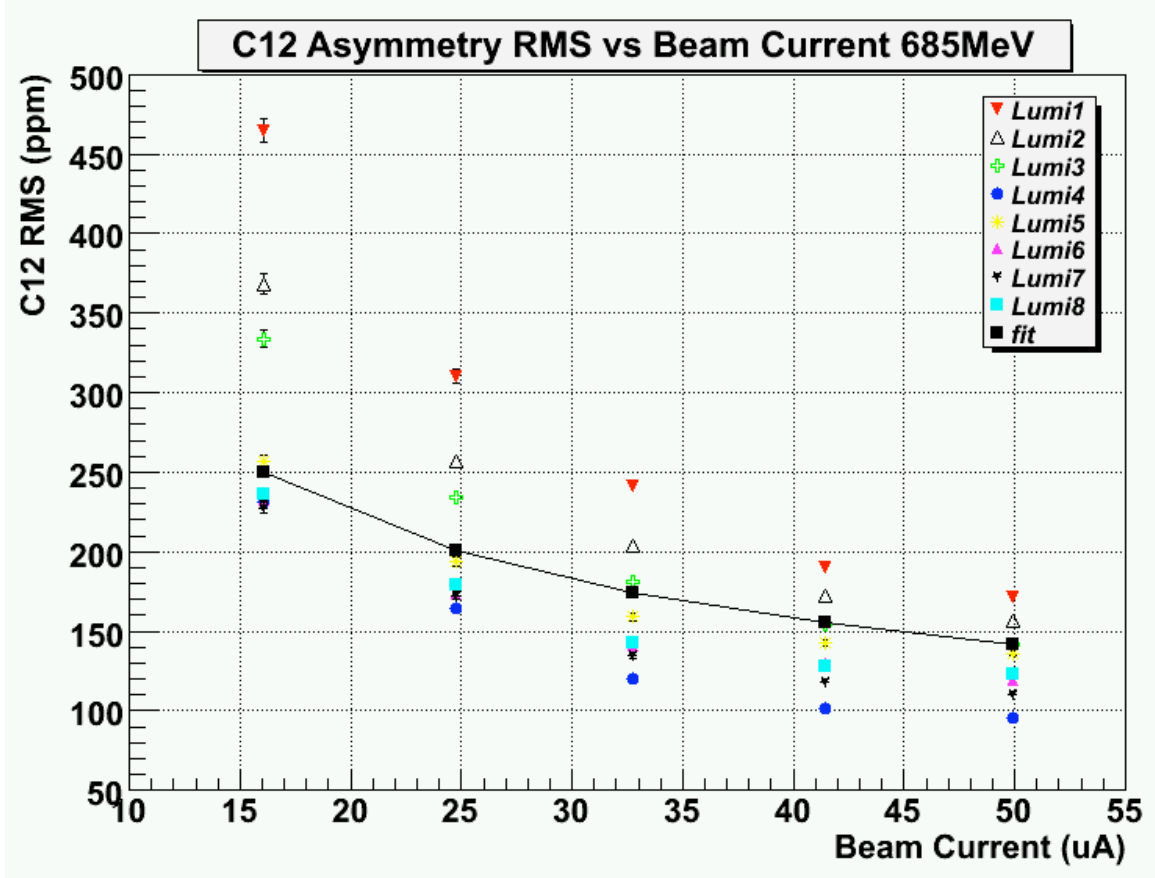

Figure B.9: Measured asymmetry widths for a carbon target at $687 \mathrm{MeV}$. A counting statistics fit for Lumi3 is shown.

Lumi asymmetry widths for a carbon target are shown in Fig. B.9. Lumis 4-8 roughly follow a $1 / \sqrt{I_{\text {beam }}}$, consistent with counting statistics, and implying little to no noise. Lumis 1-3 have large widths at the lowest beam current, and the increase in width beyond the $1 / \sqrt{I_{\text {beam }}}$ can be attributed to some source of noise. 
The noise can be calculated using the following expression, which accounts for the noise associated with each of the MPS that make up a quartet,

$$
W_{\text {noise }}=\frac{\sqrt{4 \sigma^{2}}}{4 V_{s}}
$$

where $\sigma$ is the noise fluctuation and $V_{s}$ is the output signal voltage [Liu06]. For the worst case, Lumi1 at $16 \mu A$, the output voltage was $0.45 \mathrm{~V}$ and $W_{\text {noise }} \equiv$ $\sqrt{470^{2}-285^{2}}=373 \mathrm{ppm}$. This results in $\sigma=0.37 \mathrm{mV}$. Similarly, the least noisy Lumi, Lumi5, has $\sigma=0.068 \mathrm{mV}$. The Advanced Research Instruments preamps used with Lumis 5-8, were designed as a low noise preamp whereas the Hamamatsu preamps were lower quality instruments. Additionally, several of the R375 photomultiplier tubes were used for the forward angle measurement either actively or as spares, while two of the tubes were newly purchased. It is therefore not surprising that the noise associated with each detector varied significantly. Extrapolating the worst case noise fluctuation (Lumi1) to the backward angle measurement's nominal running conditions, the contribution to the asymmetry from noise is at most, 77 ppm for $687 \mathrm{MeV}$ deuterium, $44 \mathrm{ppm}$ for $362 \mathrm{MeV}$ deuterium, and $31 \mathrm{ppm}$ for all hydrogen runs.

The behavior of the Lumi asymmetry widths as a function of the beam current varied with liquid target. This is shown in Fig. B.10 for deuterium and Fig. B.11 for hydrogen. The upsweep, or increase in the widths, above $25 \mu \mathrm{A}$ can be attributed to target density fluctuations. The contributions to the widths for the hydrogen target at $57 \mu \mathrm{A}$ can be approximated in the following manner. For each Lumi, the noise at a beam current of $10.5 \mu \mathrm{A}$ is calculated as described above, and subtracted 


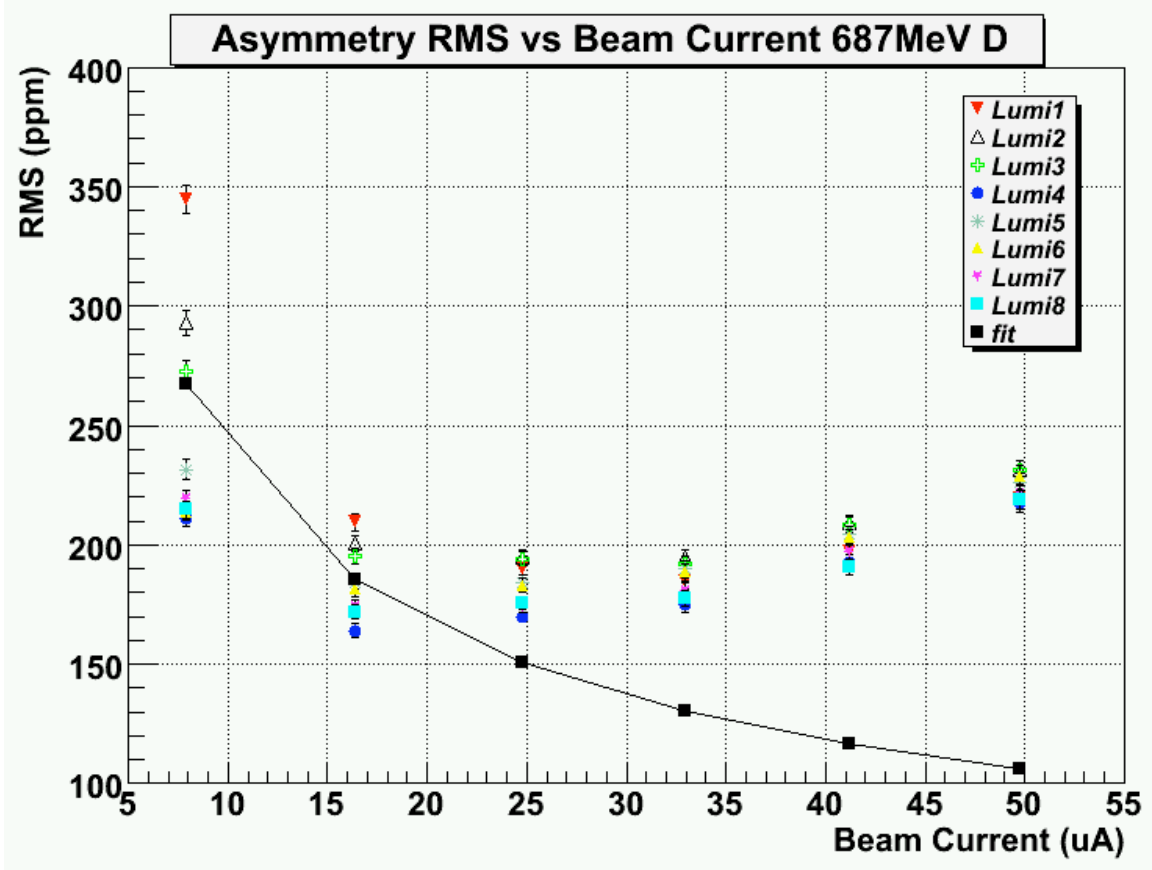

Figure B.10: Measured asymmetry widths for a liquid deuterium target at $687 \mathrm{MeV}$. A counting statistics fit for Lumi3 is shown. The difference between the fit and the data can be attributed to target density fluctuations. 


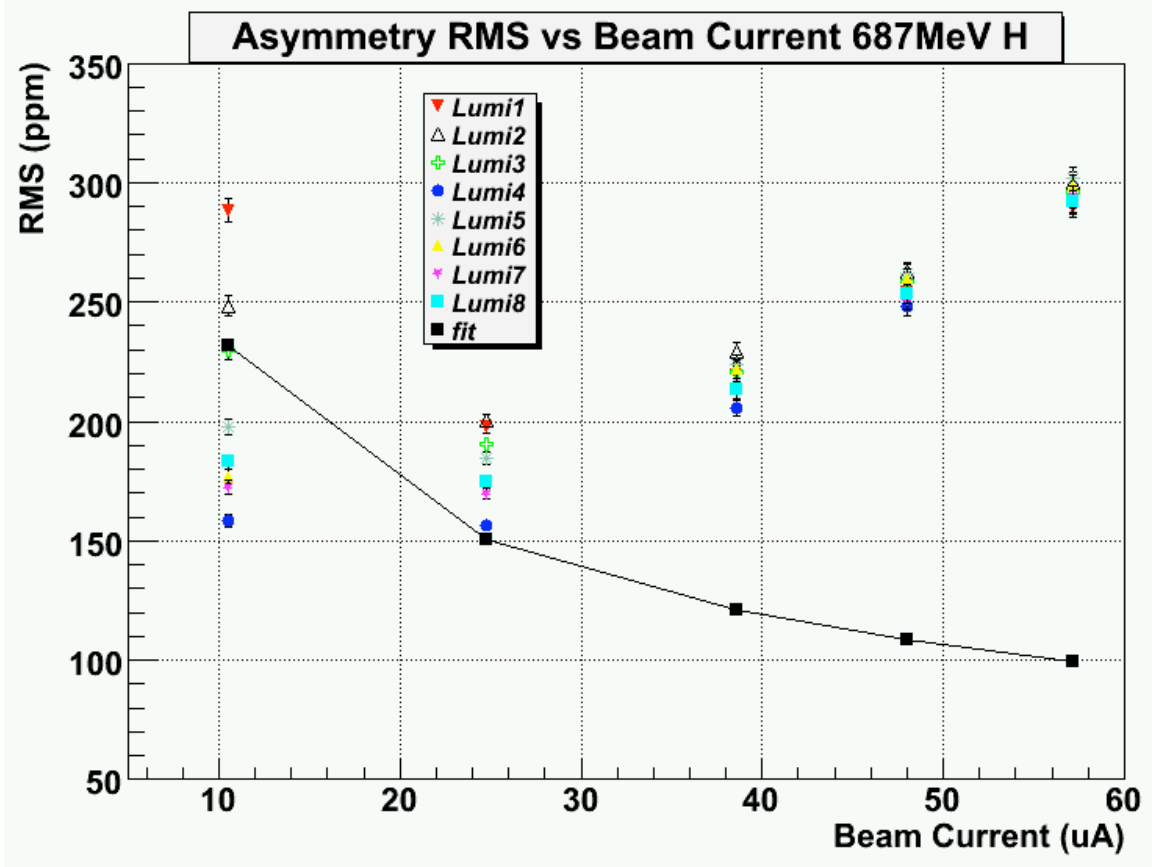

Figure B.11: Measured asymmetry widths for a liquid hydrogen target at $687 \mathrm{MeV}$. A counting statistics fit for Lumi3 is shown. The difference between the fit and the data can be attributed to target density fluctuations. 
from the measured asymmetry width at $10.5 \mu \mathrm{A}$. The same is done at, e.g., $57 \mu \mathrm{A}$. The following relationship is then used,

$$
W_{57 s t a t}=W_{10.5} \times \frac{\sqrt{I_{10.5}}}{\sqrt{I_{57}}}
$$

where $W_{57 \text { stat }}$ is the expected width at $57 \mu A$ due to counting statistics, $W_{10.5}$ is the measured width at $10.5 \mu \mathrm{A}$ less the noise contribution, and $I$ represents the beam current. Then,

$$
W_{57 b o i l}=\sqrt{W_{57}^{2}-W_{57 s t a t}^{2}},
$$

where again, $W_{57}$ is the measured width at $57 \mu A$ less the noise contribution. This results in contributions to the asymmetry widths from target density fluctuations for Lumi1 equal to $271 \pm 15 \mathrm{ppm}$ and for Lumi5 of $290 \pm 15 \mathrm{ppm}$. Using a similar technique for high energy deuterium, the contributions to the widths at $25 \mu \mathrm{A}$ is $93 \pm 15$ ppm for Lumi1 and $113 \pm 15$ ppm for Lumi5.

The contribution of target density fluctuations to the asymmetry width will be the same for all of the detectors, i.e. for $687 \mathrm{MeV}$ hydrogen, the contribution to the asymmetry width for the main detectors will also be $\sim 280 \mathrm{ppm}$. But whereas the typical width of the Lumi asymmetries is $\sim 300 \mathrm{ppm}$, by comparison, asymmetry widths for one octant of the elastic electron locus was of the order of 19,000 ppm. Therefore, although target density fluctuations contribute to the asymmetry widths, the contribution is negligible when compared to a typical asymmetry width measured for one octant. 


\section{B.5 Spin Calculation}

The placement of the Lumis around the beam line, as shown in Fig. B.1, allows a look at the Lumis as a function of the angle $\phi$ around the beam axis. The two sets of detectors (Lumis 1-4 and Lumis 5-8) are actually separated by $\sim 23 \mathrm{~cm}$, but that separation results in a small difference in acceptance, so the Lumis will be treated as lying in the same plane. The rest of this appendix will refer to the Lumis by the octant they are in as shown in Fig. B.12, rather than by their respective detector number.

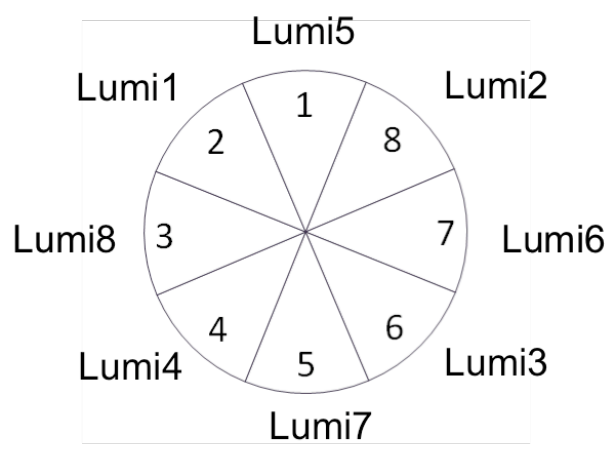

Figure B.12: Illustration of the numbering of the octants (numbers inside the circle) and the corresponding Lumi monitor. The beam is going into the page.

With a longitudinally polarized beam, the Lumis should not have any asymmetry rate dependence on $\phi$ unless there is a misalignment of the spin direction. The following simple geometric relationship, for the misalignment angle $\alpha$ of the angle between the spin and the beam direction,

$$
\sin \alpha=\frac{A_{L}}{A_{T}}
$$

provides a quick and robust means to calculate, $\alpha$, where $A_{L}\left(A_{T}\right)$ is the detected Lumi longitudinal (transverse) asymmetry. The Lumi longitudinal asymmetries 
were small, on the order of $0.75 \mathrm{ppm}$ as shown in Fig B.13, with the transverse asymmetries being much larger, on the order of 19 ppm as shown in Fig B.14. The asymmetries used in the calculation were blinded, but because asymmetries with the same target and energy had the same blinding factor, the blinding factors where eliminated when the ratio was taken.

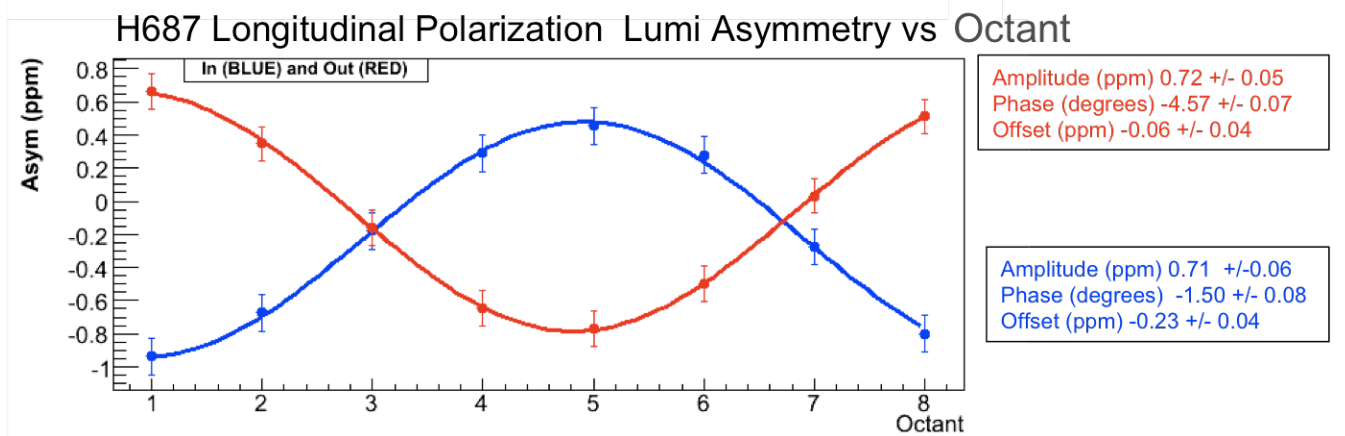

Figure B.13: Lumi asymmetries (blinded) versus octant for longitudinally polarized electrons scattered at $687 \mathrm{MeV}$ from an unpolarized hydrogen target.

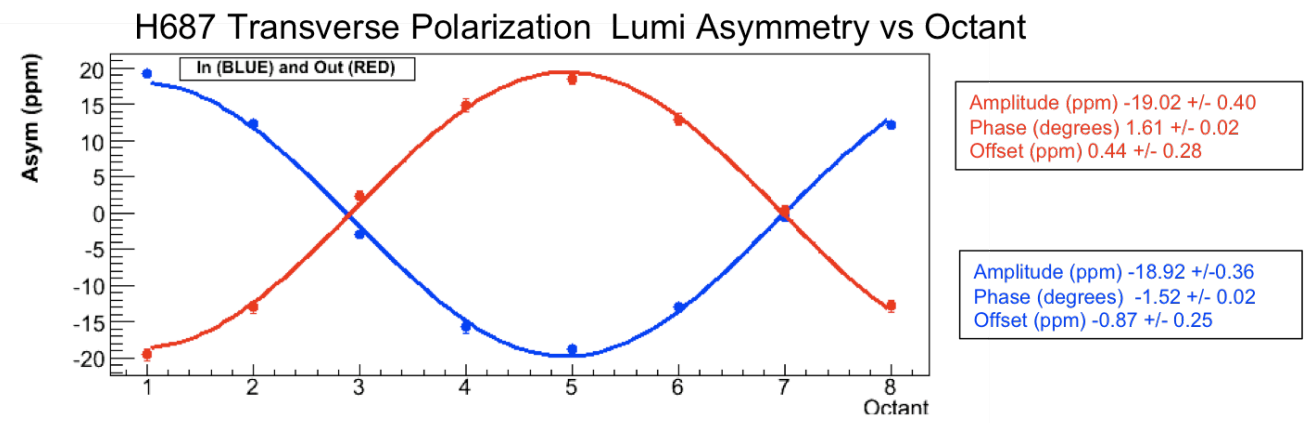

Figure B.14: Lumi asymmetries (blinded) versus octant for transversely polarized electrons scattered at $687 \mathrm{MeV}$ from an unpolarized hydrogen target.

The electrons are generated with a polarization, but as the electron beam is transported around the accelerator, the beam is bent by a series of dipole magnets that cause the beam's polarization to precess. The amount of precession, $\phi_{\text {spin }}$, is proportional to the beam energy and the angle the beam is bent through, $\theta_{\text {bend }}$, and 
Table B.6: Spin angle for nominal longitudinally polarized electron beam calculated using Lumi asymmetries.

\begin{tabular}{|l|c|c|c|}
\hline \hline Run Period & Wien Angle & $\alpha_{\text {spin }}($ degrees $)$ & $\alpha_{\text {spin }}$ err (degrees) \\
\hline \hline H 687 & $92.3 \pm 2.0$ & 2.16 & 0.12 \\
H 362 & $71.2 \pm 2.0$ & 0.92 & 0.11 \\
D 362 & $69.2 \pm 2.0$ & 1.86 & 0.06 \\
D 687 & $91.5 \pm 2.0$ & 1.15 & 0.07 \\
\hline \hline
\end{tabular}

is expressed as

$$
\phi_{\text {spin }}=\frac{g-2}{2 m_{e}} \times E_{\text {beam }} \times \theta_{\text {bend }},
$$

where $g-2$ is the electron's anomalous magnetic moment, $m_{e}$ is the electron mass, and $E_{\text {beam }}$ is the beam energy. A Wien filter in the injector region of the accelerator is used to compensate for this precession. The filter consists of perpendicular electric

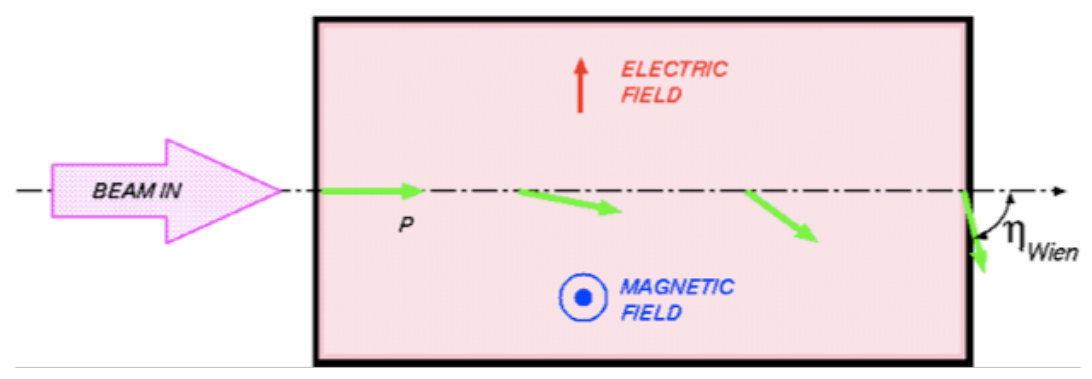

Figure B.15: The Wein filter's rotation of an electron's spin.

and magnetic fields set so that the net force on the electrons is zero, but such that the magnetic field cancels the spin precession (see Fig. B.15) and ensures that the electrons are longitudinally polarized when they reach the target. The Wien filter used in Hall $\mathrm{C}$ at TJNAF has an uncertainty of \pm 2 degrees. Table B.6 shows the spin angle calculation using Eqn. B.9 for each target and beam energy combination. There were Wien angle changes during the running periods, but all of the changes 
were within a nominal setting $\pm 2^{\circ}$. The nominal setting for the high energy runs was $\approx 92^{\circ}$ and for the low energy runs, $\approx 70^{\circ}$.

The spin angle calculation using the Lumis agrees with the value obtained during the Møller polarimetry runs [GH08], within the uncertainty of the Wien angle. The Lumis provided an excellent cross check of the quality of our beam polarization. 


\section{Appendix C}

\section{The $G^{0}$ Database}

\section{C.1 Introduction}

The $G^{0}$ database structure was developed for the $G^{0}$ forward angle experiment and then updated for the backward angle measurement. The purpose of the database was to serve as the central repository for all of the data recorded during the running of the experiment. The information was stored as summary information on a runby-run basis. The forward angle database was designed and maintained by $\mathrm{D}$. Spayde [Spa06] using a relational database management system (RDBMS) with a structure query language (SQL) user interface. The specific RDBMS used was MySQL [MyS], which is an open source application. This appendix documents the database structure at the time of the backward angle running.

\section{C.2 Database Design and Structure}

$G^{0}$ database entries fell into two main categories: physics analysis and experimental conditions. The experimental conditions included items such as target temperature, SMS current, and which target was in use (see Fig. C.1). These items were well-defined and did not change during the course of a run. The information that fell in the category of "physics analysis" included information generated from 
the various detectors and beam condition monitors during the course of an experimental run as well as any information that was the result of a calculation (i.e. averages, totals, etc).

A MySQL database is a collection of tables where the columns specify what data will be stored, and the rows contain the actual data. Each table typically has an "id" column that automatically increments each time a new entry is added to the table and which serves as the table's primary key by providing a unique identifier for each row within a table.

The $G^{0}$ database was designed so that it could easily be expanded at any time in order to record additional information necessary for the data analysis. The database was also designed to be self-documenting through the use of information tables that stored information describing data in other tables, such as measurement type (i.e. yield, asymmetry, position, etc), or units (i.e. ppm, $\mu \mathrm{A}$, etc).

The two central tables in the $G^{0}$ database were the "run" table (see Figs. C.2, C.4, and C.5) and the "analysis" table (see Figs. C.2 and C.3). The run table consisted of information describing experimental conditions, such as type of run (i.e. production, transverse, etc), data quality, and what the data set was "good for". Each row in the analysis table was associated with one run, and included information of the type "physics analysis" such as the number of good events, and information on which corrections were made to the data based on which replay engine analysis pass (see Sec. 5.1.1) was completed. Every row of information in the other database tables could be related to at least one row of data in both of these tables. Although both the run and analysis tables were central to the structure of the database, it 


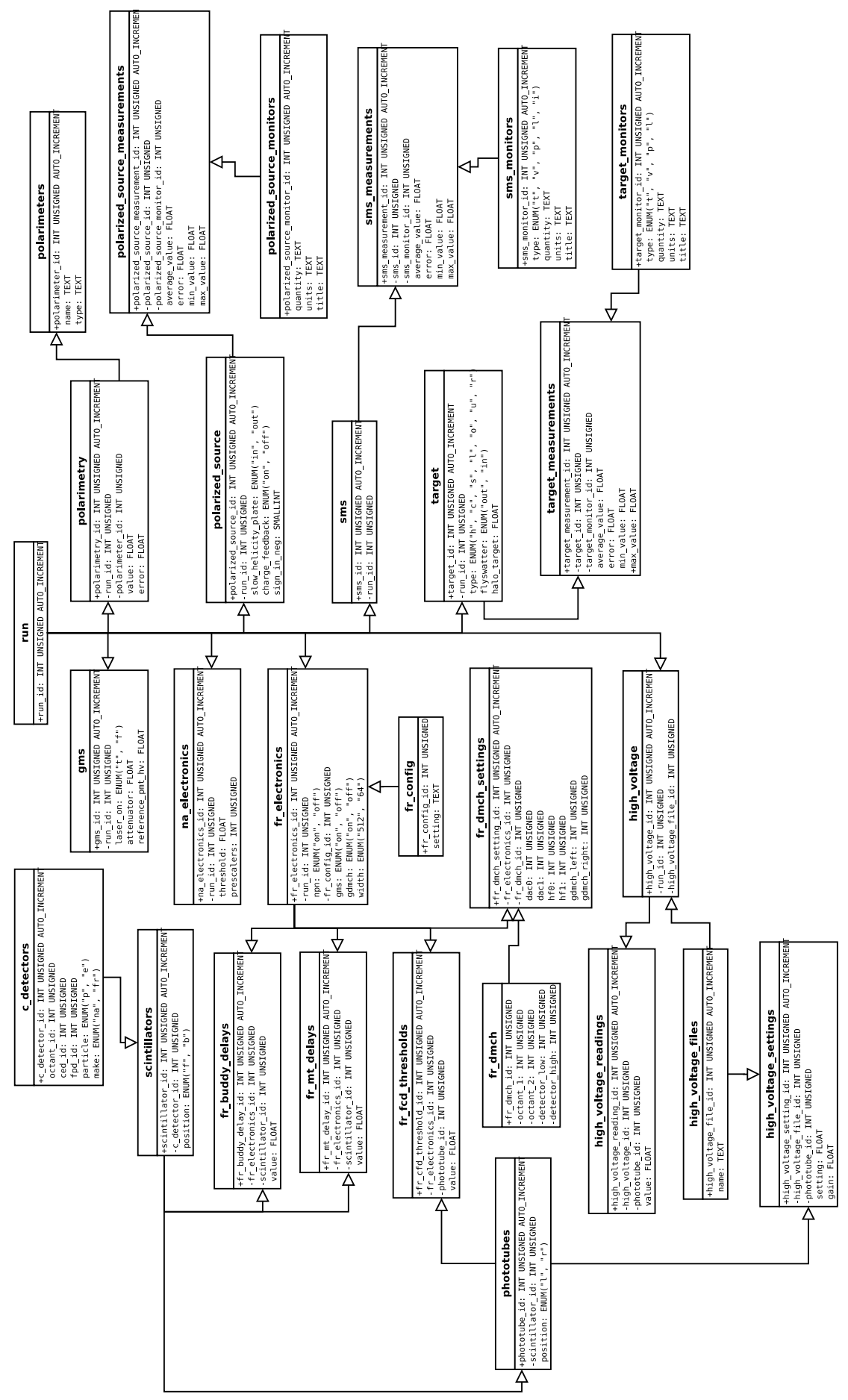

Figure C.1: Database tables to store the electronics configurations, polarized source settings and slow controls information (e.g. magnet current and target temperature). These tables are keyed off of the run id in the the run table. 
was the run table from which all other tables were derived. For any given run, there could have been multiple analysis replays, and therefore multiple rows in the analysis table, but for each analysis row, there was one and only one associated run.

For the backward angle measurement there was one master database, one unindexed slave back-up, and one indexed slave database. MySQL has a feature that allows automatic updating of any slave database whenever a change is made to the master. The $G^{0}$ master database was the only database that had data written into its fields from analysis software, or from individual inputs (i.e. calibration data). The indexed (described below) slave was used by the collaboration to analyze data, and it was also used by the $G^{0}$ replay engine when it was necessary to read information from the database prior to completing an analysis pass. The unindexed slave back-up was a mirror copy of the master database and wasn't used by the collaboration other than to automatically write a copy of itself once a week to the master silo storage system at Jefferson Laboratory.

Indexes are a feature of MySQL designed to speed queries. When a column is linked to one or more columns in other tables, MySQL builds a lookup index that allows fast retrieval of the information linked through the index. The downside to indexing is that the separate block of information created for each query increases the overall size of the database, and the index requires updating every time new information is added to the database. The slave database that was used to run queries was the indexed slave. Sometimes $G^{0}$ database queries were made after the analysis was completed to retrieve the results of the analysis replays. Other times queries were made during the actual analysis of the data. The $G^{0}$ replay engine was 
the data analysis tool described in Sec. 5.1 that summarized the pertinent information from each run, made corrections to the data where appropriate, and wrote the information to the database. Also previously discussed was the fact that the data were replayed in four passes and that passes 2, 3, and 4 all required information from earlier passes in order to complete the analysis for the given pass. This required the Replay Engine to not only write to the database, but to read and write. The replay engine was designed to use the indexed slave for any database "reading" and to use the master when it was ready to write to a database. 


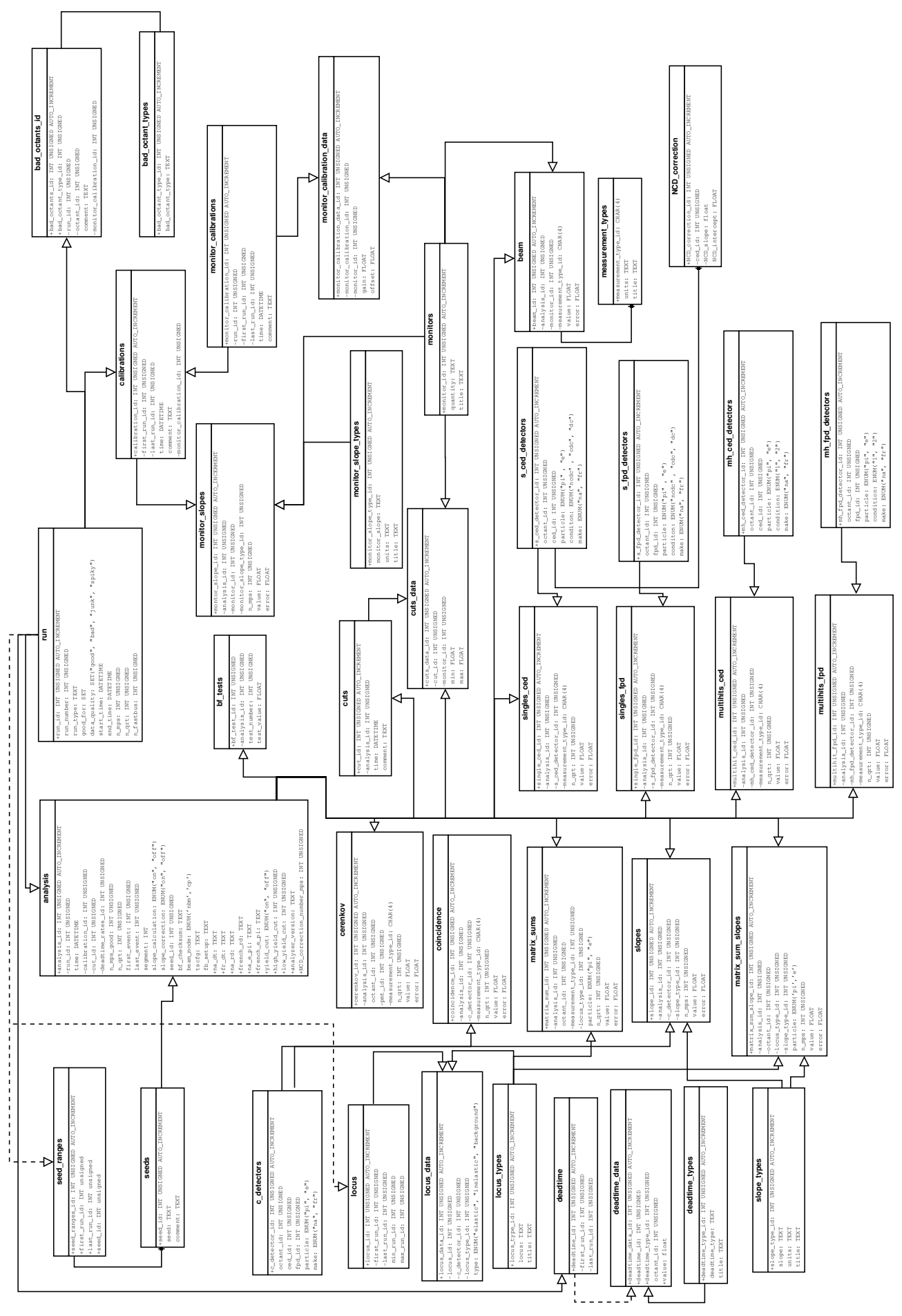

Figure C.2: Diagram of the complete analysis portion of the database. Figs. C.3, C.4, and C.5 show expanded views of this diagram. 


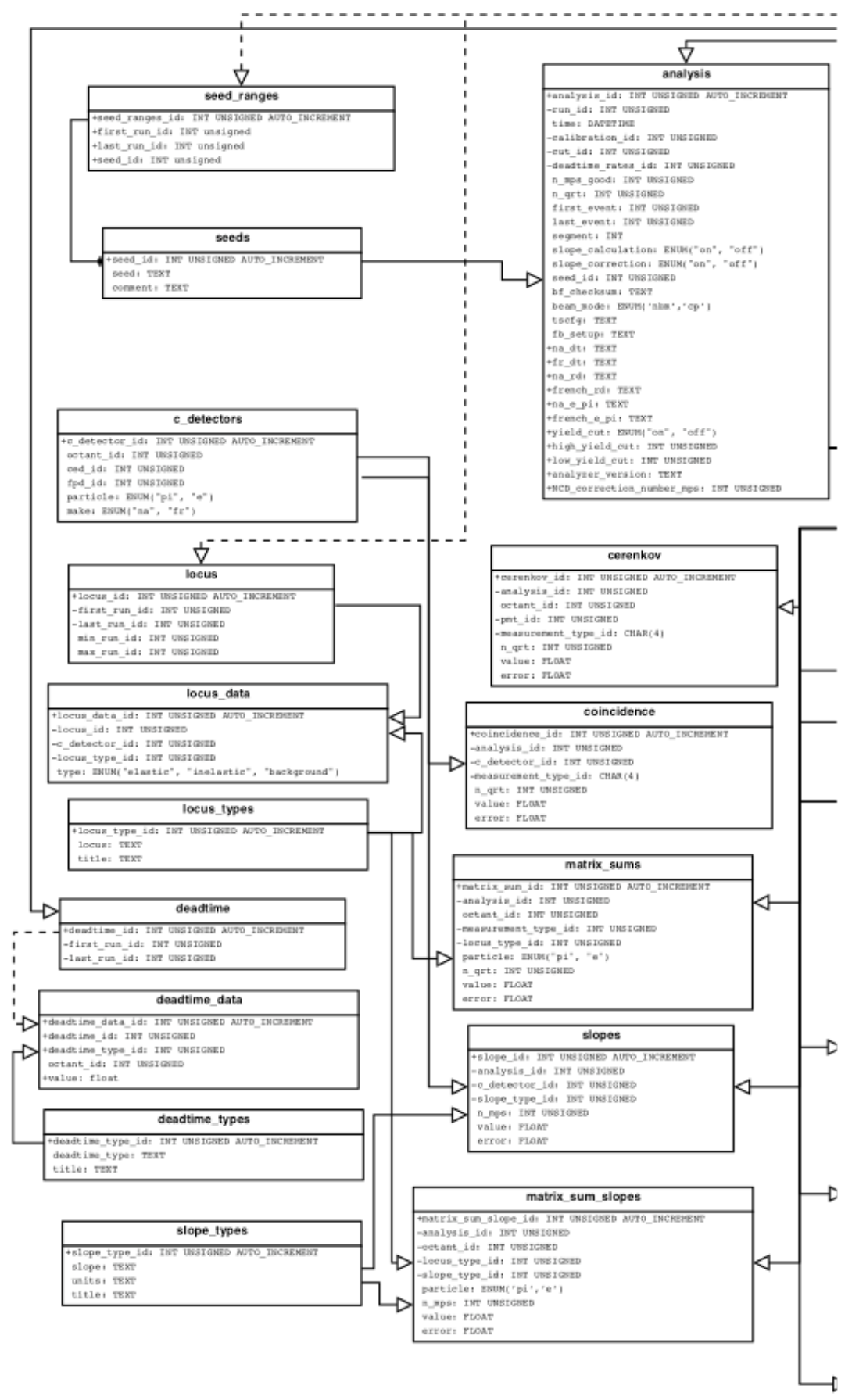

Figure C.3: The left-hand portion of Fig. C.2 showing the analysis table, locus, deadtime and slope tables as well as the seed tables and several detector tables. 


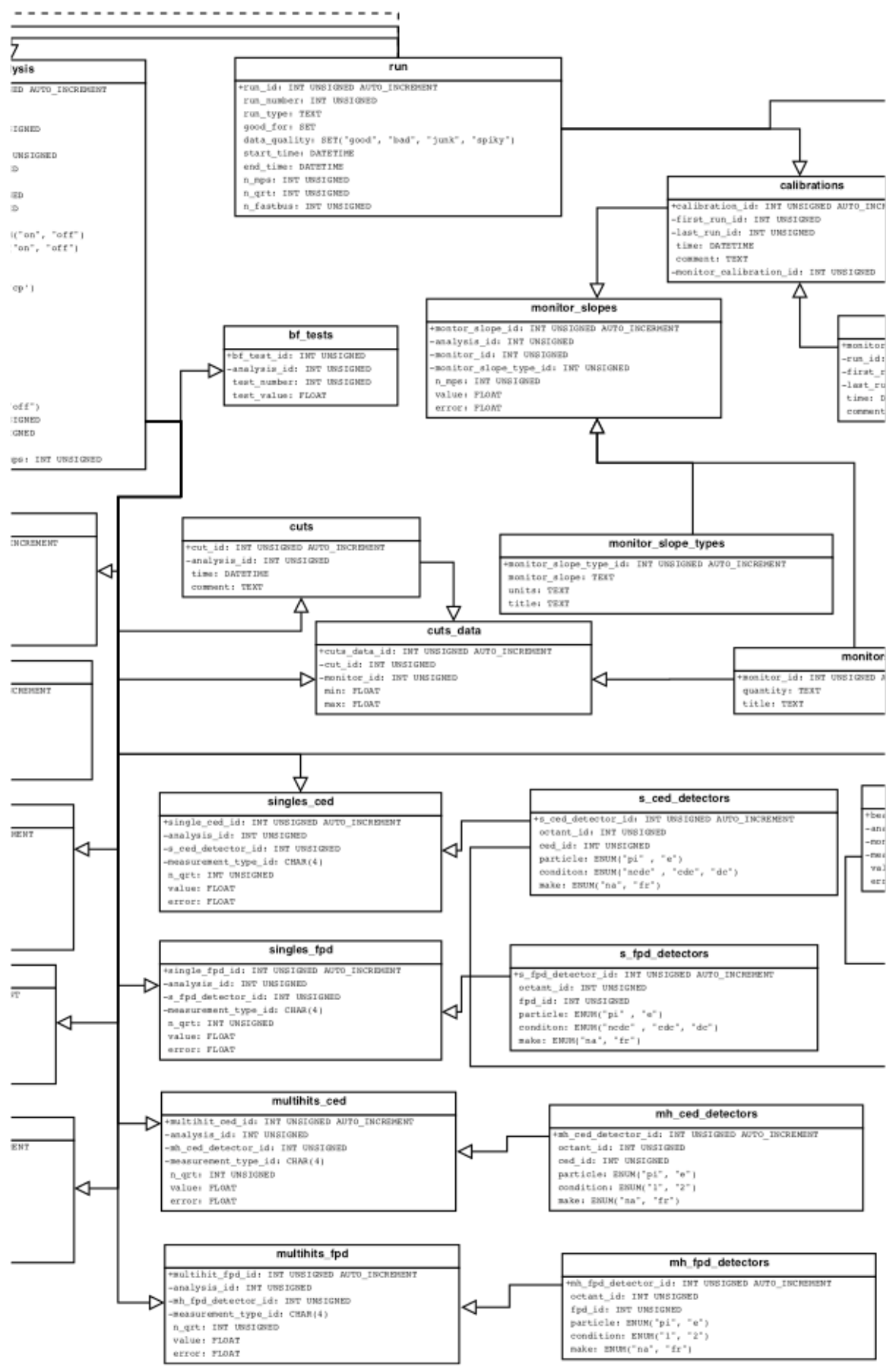

Figure C.4: The middle portion of Fig. C.2 showing the run table and all of the tables holding detector singles and multi-hit information. Also shown are the cuts tables and monitor slopes tables. 


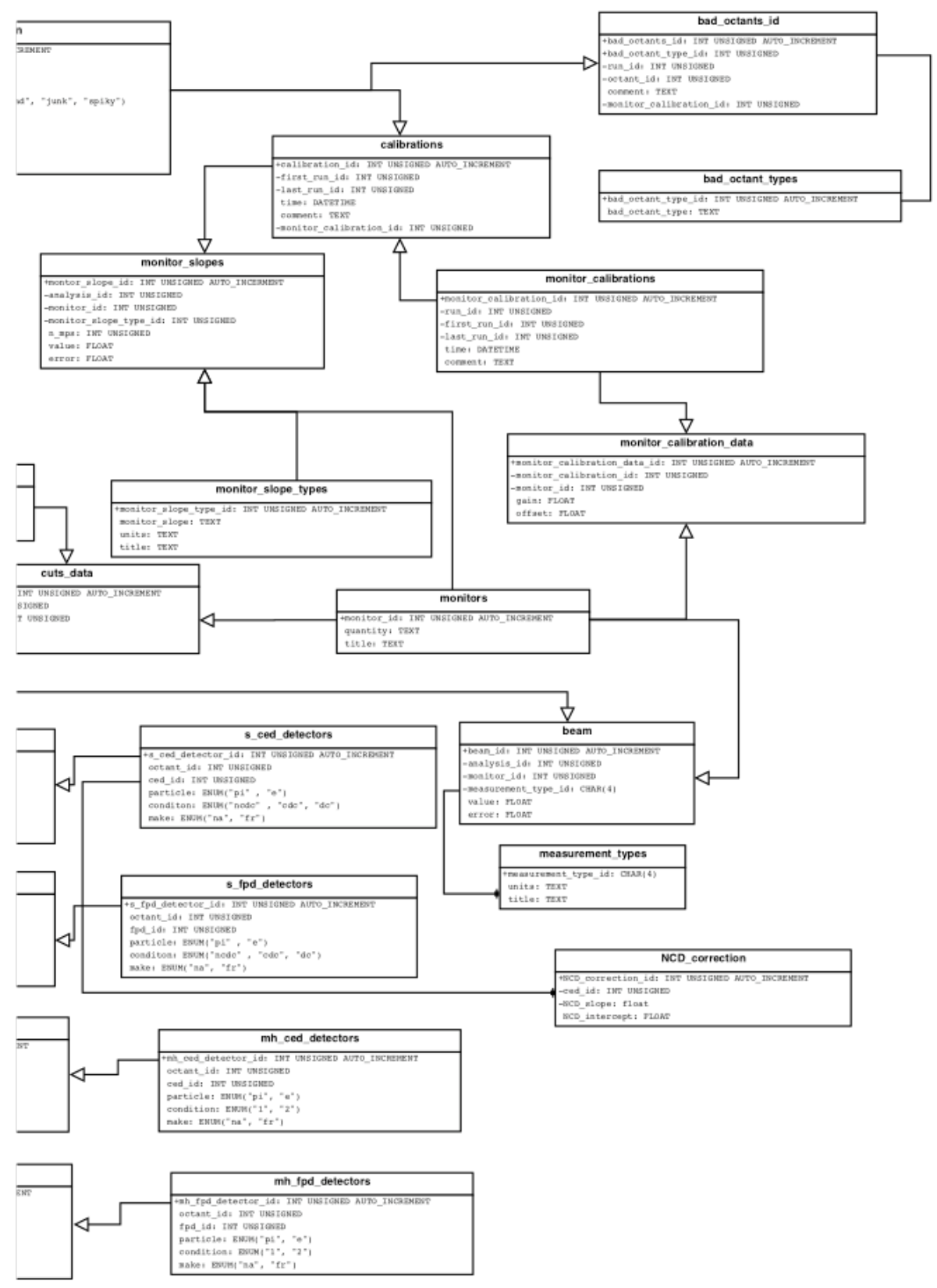

Figure C.5: The right-portion of Fig. C.2 showing all of the monitor and calibrations tables, tables with bad octant information and several of the detector tables. 


\section{Bibliography}

$\left[\mathrm{A}^{+}\right] \quad$ D. Armstrong et al. The $Q_{\text {weak }}$ Experiment: A Search of New Physics at the TeV Scale Via a Measurement of the Proton's Weak Charge. JLAB-PROPOSAL-E-05-008.

[A ${ }^{+}$99a $] \quad$ T. Adams et al. Strange content of the nucleon $(\mathrm{NuTeV})$. 1999. hepex/9906038.

[A ${ }^{+}$99b] K. A. Aniol et al. Measurement of the neutral weak form factors of the proton. Phys. Rev. Lett., 82:1096-1100, 1999.

$\left[\mathrm{A}^{+} 00\right] \quad \mathrm{T}$. Adams et al. Recent QCD results from NuTeV/CCFR Collaboration. Nuclear Physics B - Proceedings Supplements, 86(1-3):93 - 101, 2000. Proceedings of the QCD 99 Euroconference.

$\left[\mathrm{A}^{+} 01\right] \quad$ K. A. Aniol et al. New measurement of parity violation in elastic electron-proton scattering and implications for strange form factors. Physics Letters B, 509(3-4):211 - 216, 2001.

$\left[\mathrm{A}^{+} 04\right] \quad$ K. A. Aniol et al. Parity-violating electroweak asymmetry in ep scattering. Phys. Rev. C, 69(6):065501, Jun 2004.

$\left[\mathrm{A}^{+} 05\right]$ D. S. Armstrong et al. Strange-Quark Contributions to ParityViolating Asymmetries in the Forward G0 Electron-Proton Scattering Experiment. Phys. Rev. Lett., 95(9):092001, Aug 2005.

$\left[\mathrm{A}^{+} 06 \mathrm{a}\right] \quad$ E. S. Ageev et al. Gluon polarization in the nucleon from quasi-real photoproduction of high-p(T) hadron pairs. Phys. Lett., B633:25-32, 2006.

$\left[\mathrm{A}^{+} 06 \mathrm{~b}\right] \quad$ K. A. Aniol et al. Parity-violating electron scattering from He-4 and the strange electric form factor of the nucleon. Phys. Rev. Lett., 96:022003, 2006 .

[A $\left.\mathrm{A}^{+} 06 \mathrm{c}\right] \quad$ K.A. Aniol et al. Constraints on the nucleon strange form factors at $Q^{2} \sim 0.1$ gev $^{2}$. Physics Letters B, 635(5-6):275 - 279, 2006.

$\left[\mathrm{A}^{+} 06 \mathrm{~d}\right] \quad$ P.L. Anthony et al. Measurements of the $Q^{2}$-Dependence of the Proton and Neutron Spin Structure Function g1p and g1n. http://arxiv. org/abs/hep-ph/0007248v1, 2006.

$\left[\mathrm{A}^{+} 07\right] \quad$ A. Acha et al. Precision Measurements of the Nucleon Strange Form Factors at $Q^{2} \sim 0.1 \mathrm{GeV}^{2}$. Phys. Rev. Lett., 98(3):032301, Jan 2007.

$\left[\mathrm{Aac}^{+} 10\right]$ D. Androić et al. Strange Quark Contributions to Parity-Violating Asymmetries in the Backward Angle G0 Electron Scattering Experiment. Phys. Rev. Lett., 104(1):012001, Jan 2010. 
[Acc09] JLab Accel. Accelerator operations overview. http://www.jlab.org/ accel/ops_orientation/acc_overview.html, 2009.

[AoPDG08] C. Amsler and others (Particle Data Group). Review of particle physics. Phys. Lett., B667:1, 2008.

[Arr04] J. Arrington. Implications of the discrepancy between proton form factor measurements. Phys. Rev. C, 69(2):022201, Feb 2004.

$\left[\mathrm{B}^{+} 05\right] \quad$ M. Baylac et al. Effects of atomic hydrogen and deuterium exposure on high polarization GaAs photocathodes. Phys. Rev. ST Accel. Beams, 8(12):123501, Dec 2005.

$\left[\mathrm{B}^{+} 09 \mathrm{a}\right] \quad \mathrm{S}$. Baunack et al. Measurement of Strange Quark Contributions to the Vector Form Factors of the Proton at $Q^{2}=0.22(\mathrm{GeV} / \mathrm{c})^{2}$. Phys. Rev. Lett., 102(15):151803, Apr 2009.

$\left[\mathrm{B}^{+} 09 \mathrm{~b}\right] \quad \mathrm{S}$. Baunack et al. Measurement of Strange Quark Contributions to the Vector Form Factors of the Proton at $Q^{2}=0.22(\mathrm{GeV} / \mathrm{c})^{2}$. Phys. Rev. Lett., 102:151803, 2009.

[BABB08] A. Bodek, S. Avvakumov, R. Bradford, and H. S. Budd. Extraction of the Axial Nucleon Form Factor from Neutrino Experiments on Deuterium. J. Phys. Conf. Ser., 110:082004, 2008.

[Bai07] S. L. Bailey. Parity-Violation in Elastic Electron Scattering-A First Measurement of the Parity-Violating Asymmetry at $Q^{2}=0.631$ $(\mathrm{GeV} / \mathrm{c})^{2}$ at Backward Angle. PhD thesis, The College of William and Mary in Virginia, 2007.

[Bat04] G. Batigne. Mesure du contenu étrange du nucléon: expérience $G^{0}$. PhD thesis, Université Joseph Fourier, 2004.

[Bec89] D. H. Beck. Strange-quark vector currents and parity-violating electron scattering from the nucleon and from nuclei. Phys. Rev. D, 39(11):3248-3256, Jun 1989.

[BEG06] H. Breuer, C. Ellis, and T.R. Gentile. Neutron Detection Studies in Bare Photo Tubes Using Cold Neutrons at NIST. Technical Report PP 06-052, University of Maryland, College Park, September 2006.

[Bei05] Elizabeth J. Beise. The axial form factor of the nucleon. Eur. Phys. J., A24S2:43-46, 2005.

[Bei08] E. Beise. Deuterium Asymmetry Calculation, 2008. Technical Report, G0 Document Database, 770-v1.

[Ben06] F. Benmokhtar. A Quick Guide to the $G^{0}$ Data Acquisition System, 2006. Technical Report, G0 Document Database, 623-v11. 
[BFHM98] V. Bernard, H. W. Fearing, T.R. Hemmert, and U. Meiner. The form factors of the nucleon at small momentum transfer. Nuclear Physics A, 635(1-2):121 - 145, 1998.

[BH55] H.A. Bethe and F. De Hoffmann. The Mesons and Fields. Row, Peterson and Company., 1955.

[BH01] Douglas H. Beck and Barry R. Holstein. Nucleon structure and parityviolating electron scattering. Int. J. Mod. Phys., E10:1-41, 2001.

[Bjo66] J.D. Bjorken. Applications of the Chiral U(6) $\times \mathrm{U}(6)$ Algebra of Current Densities. Phys. Rep, 148(1467), 1966.

[Bjo70] J.D. Bjorken. Inelastic Scattering of Polarized Leptons from Polarized Nucleons. Phys. Rev, D1(1376), 1970.

[BM01] D. H. Beck and R. D. McKeown. Parity-violating electron scattering and nucleon structure. Ann. Rev. Nucl. Part. Sci., 51:189-217, 2001.

[BMT05] P. G. Blunden, W. Melnitchouk, and J. A. Tjon. Two-photon exchange in elastic electron nucleon scattering. Phys. Rev., C72:034612, 2005.

[BN37] F. Bloch and A. Nordsieck. Note on the Radiation Field of the Electron. Phys. Rev., 52(2):54-59, Jul 1937.

[BPS05] E. J. Beise, M. L. Pitt, and D. T. Spayde. The SAMPLE experiment and weak nucleon structure. Prog. Part. Nucl. Phys., 54:289-350, 2005.

[BR03] P. R. Bevington and D. K. Robinson. Data Reduction and Error Analysis for the Physical Sciences. McGraw Hill, third edition, 2003.

$\left[\mathrm{C}^{+} 97\right] \quad \mathrm{R}$. Carr et al. A precision measurement of the weak mixing angle in møller scattering, 1997. SLAC-PROPOSAL-E-158.

$\left[\mathrm{C}^{+} 04\right] \quad$ Y. Chao et al. Achieving Beam Quality Requirements for Parity Experiments at Jefferson Lab. http://www1.jlab.org/Ul/Publications/ view_pub.cfm?pub_id=5467, 2004.

$\left[\mathrm{C}^{+} 07\right] \quad$ C. B. Crawford et al. Measurement of the proton electric to magnetic form factor ratio from ${ }^{1} \mathrm{H}$ ( e, e'p). Phys. Rev. Lett., 98:052301, 2007.

[Cap08] C. Capuano. RCS Tests. Technical Report, G0 Document Database, 765-v1, 2008.

[CD71] T. P. Cheng and Roger Dashen. Low-Energy Photoproduction and the Chiral-Transformation Property of the Electromagnetic Current. Phys. Rev. D, 4(5):1561-1564, Sep 1971. 
[CFN93] Thomas D. Cohen, Hilmar Forkel, and Marina Nielsen. Just how strange? Loops, poles and the strangeness radius of the nucleon. Phys. Lett., B316:1-6, 1993.

[Co05] S.D. Covrig and other. The cryogenic target for the G0 experiment at Jefferson lab. Nuclear Instruments and Methods in Physics Research Section A: Accelerators, Spectrometers, Detectors and Associated Equipment, 551(2-3):218 - 235, 2005.

[Cop09] A. F. C. Coppens. Pion Contamination Correction. Technical Report, G0 Document Database, 819-v1, 2009.

[Cop10] A. F. C. Coppens. Parity violating asymmetries in the $G^{0}$ experiment; Pion photoproduction on the $\Delta$ resonance. Technical Report, G0 Document Database, 857-v1, 2010.

[CPP05] R. Carlini, S. Paige, and M. Pitt. $G^{0}$ Beam Specifications for Back Angle Running , 2005. Technical Report, G0 Document Database, 640-v1.

$\left[\mathrm{D}^{+}\right.$09a $]$Takumi Doi et al. Nucleon strangeness form factors from $\mathrm{N}_{f}=2+1$ clover fermion lattice QCD. Phys. Rev., D80:094503, 2009.

$\left[\mathrm{D}^{+}\right.$09b] Takumi Doi et al. Nucleon strangeness form factors from $N f=2+1$ clover fermion lattice QCD. Phys. Rev. D, 80(9):094503, Nov 2009.

[DA03] R. Suleiman D.S. Armstrong, B. Moffit. Target Density Fluctuations and Bulk Boiling in the Hall A cryotarget. Jefferson Lab Technical Note: JLAB-TN-03-017, 2003.

[Dal09] M. Dalton. E05-009:HAPPEX-III Update. http:///hallaweb.jlab. org/collab/meeting/2009-summer/talks/12June2009_Friday/ Dalton-E05-109_12June2009.pdf, 2009. Hall A Collaboration Meeting.

[DGH92] J. F. Donoghue, E. Golowich, and Barry R. Holstein. Dynamics of the standard model. Camb. Monogr. Part. Phys. Nucl. Phys. Cosmol., 2:1-540, 1992 .

[Dow87] K. A. Dow. Deep Inelastic Scattering from ${ }^{3} H$ and ${ }^{4} H$. PhD thesis, Massachusetts Institute of Technology, 1987.

[DP95] V. Dmitrašinović and S. J. Pollock. Isospin-breaking corrections to nucleon electroweak form factors in the constituent quark model. Phys. Rev. C, 52(2):1061-1072, Aug 1995.

[DSvK01] L. Diaconescu, R. Schiavilla, and U. van Kolck. Parity-violating electron-deuteron scattering. Phys. Rev. C, 63(4):044007, Mar 2001. 
[FJ01] B. W. Filippone and Xiangdong Ji. The spin structure of the nucleon. Adv. Nucl. Phys., 26:1, 2001.

[Fur09] C. Furget. Systematic Uncertainty for RCS, 2009. Technical Report, G0 Document Database, 821-v1.

[FW03] J. Friedrich and T. Walcher. A coherent interpretation of the form factors of the nucleon in terms of a pion cloud and constituent quarks. Eur. Phys. J., A17:607-623, 2003.

$\left[\mathrm{G}^{+} 71\right] \quad$ S. Galster et al. Elastic electron - deuteron scattering and the electric neutron form-factor at four momentum transfers 5 - $\mathrm{fm}^{-2}<Q^{2}<14-$ $\mathrm{fm}^{-2}$. Nucl. Phys., B32:221-237, 1971.

$\left[\mathrm{G}^{+} 00\right] \quad$ Y. Goto et al. Polarized parton distribution functions in the nucleon. Phys. Rev. D, 62(3):034017, Jul 2000.

[gC98] The $G^{0}$ Collaboration. PAC Jeopardy Proposal, The $G^{0}$ Experiment (Originally E91-017), 1998. Technical Report, G0 Document Database, 62-v1.

[GH08] D. Gaskell and T. Horn. Beam Polarization for $G^{0}$ Backward Angle , 2008. Technical Report, G0 Document Database, 804-v1.

[GI97] Paul Geiger and Nathan Isgur. Strange hadronic loops of the proton: A quark model calculation. Phys. Rev., D55:299-310, 1997.

[GL85] J. Gasser and H. Leutwyler. Chiral Perturbation Theory: Expansions in the Mass of the Strange Quark. Nucl. Phys., B250:465, 1985.

[GLS91] J. Gasser, H. Leutwyler, and M.E. Sainio. Form factor of the $\sigma$-term. Physics Letters B, 253(1-2):260 - 264, 1991.

[Gou06] G. Goulven. ARS Analysis for the $G^{0}$ Experiment, 2006. Technical Report, G0 Document Database, 667-v2.

[Gra06] J Grames. Polarized Electron Sources - Special Topics Seminar at U. Va. http://www.jlab.org/accel/inj_group/doc1.htm, 2006.

[Gui05] B. Guillon. Čerenkov counter for the G0 backward angle measurements. Eur. Phys. J., A24S2:145, 2005.

$\left[\mathrm{H}^{+} 76\right] \quad$ G. Höller et al. Analysis of electromagnetic nucleon form factors. Nucl. Phys., B114, 1976.

[HM84] Francis Halzen and Alan D. Martin. The Quarks \& Leptons: An Introductory Course in Modern Particle Physics. John Wiley \& Sons, Inc., 1984 . 
[HP06] J. Hansknecht and M. Poelker. Synchronous photoinjection using a frequency-doubled gain-switched fiber-coupled seed laser and ErYbdoped fiber amplifier. Phys. Rev. ST Accel. Beams, 9(6):063501, Jun 2006 .

[HPD92] E. Hadjimichael, G. I. Poulis, and T. W. Donnelly. Parity-violating asymmetry in quasielastic e-d scattering. Phys. Rev. C, 45(6):26662682, Jun 1992.

[HR00] L. Hannelius and D. O. Riska. Strangeness form factors of the proton in the chiral quark model. Phys. Rev., C62:045204, 2000.

[HRM99] H. W. Hammer and M. J. Ramsey-Musolf. K anti-K continuum and isoscalar nucleon form factors. Phys. Rev., C60:045204, 1999.

$\left[\mathrm{I}^{+} 04\right]$ T. M. Ito et al. Parity-Violating Electron Deuteron Scattering and the Proton's Neutral Weak Axial Vector Form Factor. Phys. Rev. Lett., 92(10):102003, Mar 2004.

[Jaf89] R. L. Jaffe. Stranger Than Fiction: The Strangeness Radius and Magnetic Moment of the Nucleon. Phys. Lett., B229:275, 1989.

[Ji03] X. Ji. Quark orbital angular momentum and generalized parton distributions. Int. J. Mod. Phys., A18:1303-1309, 2003.

[KCL09] S. E. Kuhn, J. P. Chen, and E. Leader. Spin Structure of the Nucleon - Status and Recent Results. Prog. Part. Nucl. Phys., 63:1-50, 2009.

[Kel04] J. J. Kelly. Simple parametrization of nucleon form factors. Phys. Rev. C, 70(6):068202, Dec 2004.

[KHP92] W. Koepf, E. M. Henley, and S. J. Pollock. Strangeness matrix elements in the nucleon. Phys. Lett., B288:11-17, 1992.

[KM88] David B. Kaplan and Aneesh Manohar. Strange Matrix Elements in the Proton from Neutral Current Experiments. Nucl. Phys., B310:527, 1988.

[KS83] T. V. Kuchto and N. M. Shumeiko. Radiative effects in deep inelastic scattering of polarized leptons by polarized nucleons. Nuclear Physics $B, 219(2): 412-436,1983$.

[KTM75] Y. Kawazoe, G. Takeda, and H. Matsuzaki. Quasielastic electron Scattering from Nuclei. Prog. Theor. Phys., 54:1394-1408, 1975.

$\left[\mathrm{LBC}^{+} 05\right]$ D. B. Leinweber, S. Boinepalli, I. C. Cloet, A. W. Thomas, A. G. Williams, R. D. Young, J. M. Zanotti, and J. B. Zhang. Precise Determination of the Strangeness Magnetic Moment of the Nucleon. Phys. Rev. Lett., 94(21):212001, Jun 2005. 
$\left[\mathrm{LBT}^{+} 06\right]$ D. B. Leinweber, S. Boinepalli, A. W. Thomas, P. Wang, A. G. Williams, R. D. Young, J. M. Zanotti, and J. B. Zhang. Strange Electric Form Factor of the Proton. Phys. Rev. Lett., 97(2):022001, Jul 2006.

[Lee06] L. Lee. North American Cerenkov Detector Manual, 2006. Technical Report, G0 Document Database, 628-v2.

[Leo94] W. R. Leo. Techniques for Nuclear and Particle Physics Experiments. Springer-Verlag., 1994.

[Liu06] J. Liu. A Measurement of the Strange Quark Contributions to the Electromagnetic Form Factors of the Nucleon. PhD thesis, University of Maryland, College Park, 2006.

[LM99] R. Lewis and N. Mobed. Isospin violation and the proton's neutral weak magnetic form factor. Phys. Rev. D, 59(7):073002, Mar 1999.

[LMRM07] J. Liu, R. D. McKeown, and M. J. Ramsey-Musolf. Global Analysis of Nucleon Strange Form Factors at Low $Q^{2}$. Phys. Rev., C76:025202, 2007.

$\left[\mathrm{LNP}^{+}\right.$07] Hung-Liang Lai, Pavel Nadolsky, Jonathan Pumplin, Daniel Stump, Wu-Ki Tung, and Chien-Peng Yuan. The strange parton distribution of the nucleon: global analysis and applications. Journal of High Energy Physics, 2007(04):089, 2007.

[Lop96] M. Loppacher. Møller Polarimetry for CEBAF Hall C. PhD thesis, Universität Basel , 1996.

[LSS06] Elliot Leader, Aleksander V. Sidorov, and Dimiter B. Stamenov. Longitudinal polarized parton densities updated. Phys. Rev., D73:034023, 2006.

[LV91] S. A. Larin and J. A. M. Vermaseren. The $\alpha \mathrm{s} 3$ corrections to the Bjorken sum rule for polarized electroproduction and to the GrossLlewellyn Smith sum rule. Physics Letters B, 259(3), 1991.

$\left[\mathrm{M}^{+} 94\right] \quad$ M. J. Musolf et al. Intermediate-energy semileptonic probes of the hadronic neutral current. Phys. Rept., 239:1-178, 1994.

$\left[\mathrm{M}^{+} 97\right] \quad$ B. Mueller et al. Measurement of the Proton's Neutral Weak Magnetic Form Factor. Phys. Rev. Lett., 78(20):3824-3827, May 1997.

$\left[\mathrm{M}^{+} 04\right] \quad$ F. E. Maas et al. Measurement of Strange-Quark Contributions to the Nucleon's Form Factors at $Q^{2}=0.230(\mathrm{GeV} / \mathrm{c})^{2}$. Phys. Rev. Lett., 93(2):022002, Jul 2004. 
$\left[\mathrm{M}^{+} 05\right] \quad$ F. E. Maas et al. Evidence for Strange-Quark Contributions to the Nucleon's Form Factors at $Q^{2}=0.108(\mathrm{GeV} / \mathrm{c})^{2}$. Phys. Rev. Lett., 94(15):152001, Apr 2005.

$\left[\mathrm{M}^{+} 08\right]$ D. Marchand et al. G0 electronics and data acquisition (forwardangle measurements). Nuclear Instruments and Methods in Physics Research Section A: Accelerators, Spectrometers, Detectors and Associated Equipment, 586(2):251 - 269, 2008.

[Mam09a] J. Mammei. Final $Q^{2}$ report, 2009. Technical Report, G0 Document Database, 829-v3.

[Mam09b] J. Mammeii. Transverse Asymmetry Correction, 2009. Technical Report, G0 Document Database, 838-v2.

[Mam10] J. M. Mammei. Parity-Violating Elastic Electron Nucleon Scattering: Measurement of the Strange Quark Content of the Nucleon and Towards a Measurement of the Weak Charge of the Proton. 2010.

[MB94] M. J. Musolf and M. Burkardt. Stranger Still: Kaon Loops and Strange Quark Matrix Elements of the Nucleon. Zeitschrift für Physik C, 61:433, 1994.

[McK89] R. D. McKeown. Sensitivity of Polarized Elastic electron Proton Scattering to the Anomalous Baryon Number Magnetic Moment. Phys. Lett., B219:140-142, 1989.

[Mil98] Gerald A. Miller. Nucleon charge symmetry breaking and parity violating electron-proton scattering. Phys. Rev. C, 57(3):1492-1505, Mar 1998.

[MS84] W. J. Marciano and A. Sirlin. Some general properties of the $o(\alpha)$ corrections to parity violation in atoms. Phys. Rev. D, 29(1):75-88, Jan 1984.

[MT69] L. W. Mo and Y. S. Tsai. Radiative Corrections to Elastic and Inelastic ep and up Scattering. Rev. Mod. Phys., 41(1):205-235, Jan 1969.

[Mue09] M. Muether. Dilutions from Field Scan Analysis, 2009. Technical Report, G0 Document Database, 850-v11.

[MVvK00] C. M. Maekawa, J. S. Veiga, and U. van Kolck. The nucleon anapole form factor in chiral perturbation theory to sub-leading order. Physics Letters B, 488(2):167 - 174, 2000.

[MyS] MySQL. http://www.mysql.com.

[Nak05] K. Nakahara. Linear regression report for the Forward angle data. $G^{0}$ Internal Document, G0-doc-556-v1, 2005. 
[Nak06] K. Nakahara. Measurement of the Strange Magnetic Form Factor of the Proton using Elastic Electron Scattering. PhD thesis, University of Illinois, Urbana-Champaign, 2006.

[Nap91] J. Napolitano. Measuring the strangeness radius of the proton. Phys. Rev. C, 43(3):1473-1475, Mar 1991.

[NZKY09] Keitaro Nagata, Hai Qing Zhou, Chung Wen Kao, and Shin Nan Yang. Contribution of two-boson exchange with $\delta(1232)$ excitation to parity-violating elastic electron-proton scattering. Phys. Rev. C, 79(6):062501, Jun 2009.

[OM59] Haakon Olsen and L. C. Maximon. Photon and Electron Polarization in High-Energy Bremsstrahlung and Pair Production with Screening. Phys. Rev., 114(3):887-904, May 1959.

$\left[\mathrm{P}^{+} 78\right] \quad$ C.Y. Prescott et al. Parity non-conservation in inelastic electron scattering. Physics Letters B, 77(3):347 - 352, 1978.

$\left[\mathrm{P}^{+} 80\right] \quad$ D. Pierce et al. GaAs Spin Polarized Electron Source. Review of Scientific Instruments, 51, Apr 1980.

[Pas07] K. D. Paschke. Controlling helicity-correlated beam asymmetries in a polarized electron source. Eur. Phys. J., A32:549-553, 2007.

[PC01] D. T. Pierce and R. J. Celotta. Spin-Polarized Electrons. A Century of Excellence in Measurements, Standards, and Technology: A Chronicle of Selected NBS/NIST Publications 1901-2000 ed. D.R. Lide, (721.5):203-208, 2001.

[PGSH05] S. Phillips, D. Gaskell, D. T. Spayde, and T. Horn. Møller Polarimetry in the $G^{0}$ Experiment, 2005. Technical Report, G0 Document Database, 614-v1.

[Pil07] P. Pillot. Deadtime Correction, 2007. Technical Report, G0 Document Database, 679-v1.

$\left[\mathrm{R}^{+} 04\right] \quad$ K. Reza et al. CEBAF Injector Achieved World's Best Beam Quality for Three Simultaneous Beams with a Wide Range of Bunch Charges. http://www1.jlab.org/Ul/Publications/view_pub.cfm? pub_id=5466, 2004.

[Rea08] Jean-Sebastien Real. Scaler Simulation Update. Technical Report, G0 Document Database, 776-v1, 2008.

[Rio09] D.B. Rios. Parity violating electron backscattering. http: //wwwkph.kph.uni-mainz.de/A4//blog/articles/publications/ index.html\#talks, 2009. A4 Collaboration. 
[RMI97] M. J. Ramsey-Musolf and Hiroshi Ito. Chiral symmetry and the nucleon's vector strangeness form factors. Phys. Rev. C, 55(6):3066-3082, Jun 1997.

[Rol99] E. Rollinde. G0-Geant Manual. G $G^{0}$ Internal Document, G0-doc-439-v1, 1999.

[ROO] ROOT. http://root.cern.ch.

[RS74] E. Reya and K. Schilcher. Neutral-current effects in elastic electronnucleon scattering. Phys. Rev. D, 10(3):952-959, Aug 1974.

$\left[\mathrm{S}^{+} 00\right] \quad$ D. T. Spayde et al. Parity Violation in Elastic Electron-Proton Scattering and the Proton's Strange Magnetic Form Factor. Phys. Rev. Lett., 84(6):1106-1109, Feb 2000.

$\left[\mathrm{S}^{+} 04\right] \quad$ D. T. Spayde et al. The strange quark contribution to the proton's magnetic moment. Physics Letters B, 583(1-2):79 - 86, 2004.

[Sac62] R. G. Sachs. High-Energy Behavior of Nucleon Electromagnetic Form Factors. Phys. Rev., 126(6):2256-2260, Jun 1962.

[Sai94] M. E. Sainio. Pion-Nucleon Sigma Term, 1994. Invited talk at Workshop on Chiral Dynamics: Theory and Experiments, Cambridge, MA, 25-29 July 1994.

[Sai02] M. E. Sainio. Pion nucleon sigma-term: A review, 2002.

[Sch06] R. Schiavilla. private communication with E. J. Beise. 2006.

[Sch08] J. Schaub, 2008. $G^{0}$ Internal Document, ELOG:Analysis 342.

[SCP04] R. Schiavilla, J. Carlson, and M. Paris. Parity-violating interaction effects in the np system. Phys. Rev. C, 70(4):044007, Oct 2004.

[SFD05] E. Stangland, R. Flood, and H. Dong. $G^{0}$ Helicity Digital Controls, 2005. Technical Report, G0 Document Database, 572-v1.

[Sin98] C. K. Sinclair. Electron beam polarimetry. volume 451, pages 23-39. AIP, 1998.

[SKUG06] Antonio Silva, Hyun-Chul Kim, Diana Urbano, and Klaus Goeke. Parity-violating aysmmetries in elastic e(pol.) p scattering in the chiral quark-soliton model: Comparison with A4, G0, HAPPEX and SAMPLE. Phys. Rev., D74:054011, 2006.

[Spa01] D.T. Spayde. Measurement of the Strange Quark Contribution to Proton Structure Through Parity Violating Electron-Proton Scattering. $\mathrm{PhD}$ thesis, University of Maryland, College Park, 2001. 
[Spa06] D. Spayde. private communication with C. Ellis. 2006.

[TBM09] J. A. Tjon, P. G. Blunden, and W. Melnitchouk. Detailed analysis of two-boson exchange in parity-violating e-p scattering. Physical Review C (Nuclear Physics), 79(5):055201, 2009.

[Tsa71] Y.S. Tsai. Radiative Corrections to Electron Scatterings, January 1971.

[Ver08a] M. Versteegen. Cerenkov Efficiency Estimation Beam Energy $362 \mathrm{MeV}$ on $\mathrm{LD}_{2}$ target. Technical Report, G0 Document Database, 764-v1, 2008.

[Ver08b] M. Versteegen. Cerenkov Efficiency Estimation Beam Energy $687 \mathrm{MeV}$ on $\mathrm{LD}_{2}$ target. Technical Report, G0 Document Database, 765-v1, 2008.

[Ver09] M. Versteegen. Mesure par violation de parité de la contribution étrange á la structure électromagnétique du nucléon dans l'expérience $G^{0}$. PhD thesis, Université Joseph Fourier, 2009.

[W5094] CERN Program Library Long Writeup W5013. GEANT, Detector Description and simulation Tool Version 3.2.1. 1994.

[Won90] S. S. M. Wong. Introductory Nuclear Physics. P T R Prentice Hall., 1990 .

[Yak04] M. El Yakoubi. Contribution du quark étrange à la structure électromanétique du nucléon: Les résultats de l'expérience PVA4 à $Q^{2}=0.23(\mathrm{GeV} / \mathrm{c})^{2}$. PhD thesis, L'Université de Paris XI, 2004.

[ZPHRM00] S. Zhu, S. J. Puglia, B. R. Holstein, and M. J. Ramsey-Musolf. Nucleon anapole moment and parity-violating ep scattering. Phys. Rev. D, 62(3):033008, Jul 2000. 